Schmerz 2021 - 35 (Suppl 2):S73-S155

https://doi.org/10.1007/s00482-021-00581-2

Angenommen: 6 . August 2021

(c) Der/die Autor(en) 2021

\section{Internationale Klassifikation orofazialer Schmerzen (ICOP), 1. Auflage}

Deutsche Migräne- und Kopfschmerzgesellschaft e.V.

Frankfurt, Deutschland

\section{Vorwort zur deutschsprachigen Auflage der ersten International Classification of Orofacial Pain (Internationale Klassifikation orofazialer Schmerzen, ICOP)}

In 2020 wurde die erste Auflage der International Classification of Orofacial Pain (Internationale Klassifikation orofazialer Schmerzen, ICOP) veröffentlicht.

Diese erfolgte als Kooperation von internationalen Experten für orofaziale Schmerzen, u. a. der International Headache Society (IHS) sowie der International Association for the Study of Pain (IASP). Sie stellt den Versuch dar, aufbauend auf den bereits publizierten diagnostischen Kriterien für orofaziale Schmerzen in der International Classification of Headache Disorders (ICHD-3), den Diagnostic Criteria for Temporomandibular Disorders (DC/TMD) sowie der 11. überarbeiteten Fassung der International Classification of Diseases (ICD-11) ein einheitliches Regelwerk für die klinische Versorgung und wissenschaftliche Fragestellungen zu orofazialen Schmerzsyndromen zu schaffen. Ein zentrales Anliegen war die einheitliche Klassifikation unabhängig vom Ort des Auftretens.

Für die wissenschaftlichen Fachgesellschaften im deutschsprachigen Raum, die Deutsche Migräne und Kopfschmerzgesellschaft (DMKG), die Österreichische Kopfschmerzgesellschaft (ÖKSG) und die Schweizer Kopfwehgesellschaft (SKG) war dies Anlass für eine unter Federführung der DMKG rasch erstellte und bei der IHS angemeldete offizielle Übersetzung der ICOP in die deutsche Sprache, mit finanzieller Unterstützung durch die drei Gesellschaften.

Die erste Version der Übersetzung wurde von der beauftragten Übersetzerin Frau Silvia Autenrieth erstellt. Dabei wurde die Nomenklatur der teils überlappenden ICHD-3 berücksichtigt. Die Schreibweise der medizinischen Fachausdrücke erfolgte nach Pschyrembel. Die erste Version wurde von Mitgliedern aus dem DMKG-Präsidium fachlich überprüft und wo nötig angepasst (namentlich erwähnt seien hier Dr. Thomas Dresler, PD Dr. Charly Gaul, PD Dr. Gudrun Goßrau, PD Dr. Tim Jürgens, Prof. Dr. Martin Marziniak, PD Dr. Lars Neeb und Dr. Dr. Frank Sanner als externer Experte). Die anschließende redaktionelle Überarbeitung übernahm Frau Dr. Axenia Schäfer nach einer Konsensuskonferenz zur Klärung offener Fragen.

Bei allen möglichen Unklarheiten in der ICOP und trotz der von einigen als nicht unproblematisch empfundenen Überlappungen mit der ICHD-3 stellt die ICOP aus unserer Sicht dennoch einen Meilenstein auf dem Weg dar, das weite und bislang noch immer unvollständig erfasste Feld der Gesichtsschmerz-Syndrome künftig unabhängig vom genauen Ort des Auftretens anhand ihres zeitlichen Musters, von Begleitsymptomen und weiterer Details genauer zu charakterisieren. Gerade in der Zusammenarbeit von Experten verschiedener Fachgesellschaften aus sehr unterschiedlichen Disziplinen der Medizin, Zahnmedizin und Lebenswissenschaften liegt die Stärke dieser Klassifikation.

Abschließend bleibt nur zu wünschen, dass diese erste Auflage die so dringend nötigen Impulse für weitere wissenschaftliche Aktivitäten auf dem Gebiet der orofazialen Schmerzen mit dem Ziel liefert, auch die klinische Versorgung von Patienten mit orofazia- 
len Schmerzsyndromen zu verbessern. Somit ist die ICOP in ihrer ersten Auflage als „Work in progress" anzusehen. Wir laden Sie dazu ein, die deutsche Übersetzung im klinischen und wissenschaftlichen Alltag rege zu nutzen.

Tim Jürgens

Präsident der Deutschen Migräne und Kopfschmerzgesellschaft e.V.

Karin Zebenholzer

Präsidentin der Österreichischen Kopfschmerzgesellschaft

Andreas Kleinschmidt

Präsident der Schweizerischen Kopfwehgesellschaft

Mitglieder der Arbeitsgruppe zur Übersetzung der ICOP (in alphabetischer Reihenfolge)

Fachliche Überarbeitung

Thomas Dresler

Stefanie Förderreuther

Charly Gaul

Gudrun Goßrau

Tim Jürgens

Martin Marziniak

Lars Neeb

Frank Sanner

Übersetzung: Silvia Autenrieth

Redaktionelle Mitarbeit: Axenia Schäfer

Dieser Beitrag wurde mit Genehmigung der International Headache Society ins Deutsche übersetzt. Originalpublikation: International Classification of Orofacial Pain, 1st edition (ICOP) (2020) Cephalalgia Bd. 40(2) 129-221 https://doi.org/10.1177/0333102419893823, @ International Headache Society 2020

The supplement containing this article is not sponsored by industry. 
Hier steht eine Anzeige.

\section{Springer}




\section{Urheberrecht}

Das Urheberrecht liegt ausschließlich bei der International Headache Society (IHS). Die Internationale Klassifikation orofazialer Schmerzen (International Classification of Orofacial Pain; ICOP) in dieser oder nachfolgenden Auflagen darf zu wissenschaftlichen, Weiterbildungs- oder klinischen Zwecken von Institutionen, Fachgesellschaften oder Einzelpersonen frei vervielfältigt werden. Jede vollständige oder auszugsweise Wiedergabe in jedweder Form zu kommerziellen Zwecken hingegen bedarf der Genehmigung der IHS, die gegen Entrichtung einer Gebühr gewährt wird. Bitte kontaktieren Sie den Herausgeber unter der untenstehenden Anschrift.

\section{Übersetzungen}

Die IHS erlaubt ausdrücklich die gesamte oder auszugsweise Übersetzung der ICOP zu klinischen und Weiterbildungszwecken, für Feldstudien oder andere Forschungszwecke. Die Genehmigung erfolgt mit der Auflage, dass alle Übersetzungen bei der IHS anzumelden sind. Bevor eine Übersetzung in Angriff genommen wird, wird den potenziellen Übersetzern geraten, sich bei der IHS zu erkundigen, ob bereits eine Übersetzung in die anvisierte Sprache vorliegt.

Alle Übersetzer sollten sich über die Notwendigkeit des Einsatzes exakter Übersetzungsprotokolle im Klaren sein. Veröffentlichungen, die über Studien berichten, die auf komplette oder teilweise Übersetzungen der ICOP zurückgreifen, sollten auch eine kurze Beschreibung des Übersetzungsprozesses beinhalten, einschließlich der Identität der Übersetzer (die Übersetzung sollte immer von mehr als einer Person erfolgen).

Die IHS selbst erteilt keine Zustimmungen zu Übersetzungen. Diese Zustimmungen können von den nationalen Mitgliedsgesellschaften erteilt werden. Sofern solche existieren, sollte um diese ersucht werden.

\section{Das Klassifikationskomitee für orofaziale Schmerzen}

Das Komitee ist eine gemeinschaftlich kooperierende Gruppe, die sich aus Mitgliedern der Orofacial and Head Pain Special Interest Group (OFHP SIG), der International Association for the Study of Pain (IASP), dem International Network for Orofacial Pain and Related Disorders Methodology (INfORM), der American Academy of Orofacial Pain (AAOP) und der International Headache Society (IHS) zusammensetzt.

\section{Vorsitzende}

Rafael Benoliel, USA; Arne May, Deutschland; Peter Svensson, Dänemark

\section{Mitglieder der Arbeitsgruppen (in alphabetischer Reihenfolge nach der/dem Vorsitzenden)}

1. Orofaziale Schmerzen zurückzuführen auf Erkrankungen dentoalveolärer und anatomisch benachbarter Strukturen Maria Pigg, Schweden (Vorsitzende);

Alan Law, USA;
Donald Nixdorf, USA;

Tara Renton, UK;

Yair Sharav, Israel

2. Myofaszial-orofaziale Schmerzen

Peter Svensson, Dänemark (Vorsitzender);

Malin Ernberg, Schweden;

Chris Peck, Australien

3. Kiefergelenksschmerzen

Per Alstergren, Schweden (Vorsitzender);

Ghabi Kaspo, USA;

Frank Lobbezoo, Niederlande;

Ambra Michelotti, Italien

4. Orofaziale Schmerzen zurückzuführen auf eine Läsion oder Erkrankung der Hirnnerven

Lene Baad-Hansen, Dänemark (Vorsitzende);

Eli Eliav, USA;

Yoshiki Imamura, Japan

5. Orofaziale Schmerzen mit Ähnlichkeit zu primären Kopfschmerzerkrankungen

Rafael Benoliel, USA (Vorsitzender);

Paulo Conti, Brasilien;

Arne May, Deutschland

6. Idiopathische orofaziale Schmerzen

Thomas List, Schweden (Vorsitzender);

Justin Durham, England;

Jean-Paul Goulet, Kanada;

Satu Jääskeläinen, Finnland

7. Psychosoziale Beurteilung von Patienten mit orofazialen

Schmerzen

Richard Ohrbach, USA

\section{Danksagung}

Es sind keine kommerziellen Sponsorengelder bei der Erstellung dieser Internationalen Klassifikation orofazialer Schmerzen geflossen.

Wir danken Timothy Steiner für die redaktionelle Bearbeitung unter Beachtung der Prinzipien, der Struktur und des Formats der ICHD. 


\section{Inhalt}

Internationale Klassifikation orofazialer Schmerzen (ICOP), 1. Auflage

S73

S74

S74

S76

S76

S76

S76

S76

S76

S80

S80

S81

S82

S86

$\mathrm{S} 86$

S86

$\mathrm{S} 92$

S99

S104

S104

$\mathrm{S} 112$

S114

S117

S120

S121

S121

S121

$\mathrm{S} 122$

$\mathrm{S} 122$

$\mathrm{S} 123$

S123

S123

S123

S124

S124

S125

S125

S126

S126

S127

S128

Vorwort zur deutschsprachigen Auflage der ersten International Classification of Orofacial Pain (Internationale Klassifikation orofazialer Schmerzen, ICOP)

Mitglieder der Arbeitsgruppe zur Übersetzung der ICOP (in alphabetischer Reihenfolge)

Fachliche Überarbeitung

Urheberrecht

Übersetzungen

Das Klassifikationskomitee für orofaziale Schmerzen

Vorsitzende

Mitglieder der Arbeitsgruppen (in alphabetischer Reihenfolge nach der/ dem Vorsitzenden)

Danksagung

Vorwort

Anleitung zum Gebrauch der ICOP

Anwendungsprinzipien

ICOP-Code Diagnose

1 Orofaziale Schmerzen zurückzuführen auf Erkrankungen und Dysfunktionen dentoalveolärer und anatomisch benachbarter Strukturen

1.1 Zahnschmerzen

1.1.1 Schmerzen der Zahnpulpa

1.1.2 Parodontale Schmerzen

1.1.3 Gingivale Schmerzen

1.2 Schmerzen der Mundschleimhaut, der Speicheldrüse und des Kieferknochens

1.2.1 Schmerzen in der Mundschleimhaut

1.2.2 Schmerzen der Speicheldrüse

1.2.3 Schmerzen im Kieferknochen

Literatur

2 Myofaszial-orofaziale Schmerzen

2.1 Primäre myofaszial-orofaziale Schmerzen

2.1.1 Akute primäre myofaszial-orofaziale Schmerzen

2.1.2 Chronische primäre myofaszial-orofaziale Schmerzen

2.2 Sekundäre myofaszial-orofaziale Schmerzen

2.2.1 Myofaszial-orofaziale Schmerzen zurückzuführen auf eine Tendinitis

2.2.2 Myofaszial-orofaziale Schmerzen zurückzuführen auf eine Myositis

2.2.3 Myofaszial-orofaziale Schmerzen zurückzuführen auf einen Muskelspasmus

Literatur

3 Kiefergelenksschmerzen

3.1 Primäre Kiefergelenksschmerzen

3.1.1 Akute primäre Kiefergelenksschmerzen

3.1.2 Chronische primäre Kiefergelenksschmerzen

3.2 Sekundäre Kiefergelenksschmerzen

3.2.1 Kiefergelenksschmerzen zurückzuführen auf eine Arthritis

3.2.2 Kiefergelenksschmerzen zurückzuführen auf eine Diskusverlagerung

3.2.3 Kiefergelenksschmerzen zurückzuführen auf eine degenerative Gelenkerkrankung

3.2.4 Kiefergelenksschmerzen zurückzuführen auf eine Subluxation 
4 Orofaziale Schmerzen zurückzuführen auf eine Läsion oder Erkrankung der Hirnnerven

S128 4.1 Schmerzen zurückzuführen auf eine Läsion oder Erkrankung des N. trigeminus

S128 4.1.1 Trigeminusneuralgie

S131 4.1.2 Andere neuropathische Schmerzen im Innervationsgebiet des N. trigeminus

S134 4.2 Schmerzen zurückzuführen auf eine Läsion oder Erkrankung der N. glossopharyngeus

S134 4.2.1 Glossopharyngeusneuralgie

S135 4.2.2 Neuropathische Schmerzen im Innervationsgebiet des N. glossopharyngeus

S135 Literatur

S137 5. Orofaziale Schmerzen mit Ähnlichkeit zu primären Kopfschmerzerkrankungen

S137 5.1 Orofaziale Migräne

S137 5.1.1 Episodische orofaziale Migräne

S137 5.1.2 Chronische orofaziale Migräne

S138 5.2 Orofaziale Schmerzen von Spannungstyp

S138 5.3 Trigeminoautonome orofaziale Schmerzen

S138 5.3.1 Orofaziale Clusterattacken

S139 5.3.2 Paroxysmale hemifaziale Schmerzen

S139 5.3.3 Short-lasting unilateral neuralgiform facial pain attacks (SUNFA) mit kranialen autonomen Symptomen

S140 5.3.4 Hemifazialer Dauerschmerz mit autonomen Symptomen

S140 5.4 Neurovaskuläre orofaziale Schmerzen

S141 5.4.1 Kurz anhaltende neurovaskuläre orofaziale Schmerzen

S141 5.4.2 Lang anhaltende neurovaskuläre orofaziale Schmerzen

S141 Literatur

S142 6 Idiopathische orofaziale Schmerzen

6.1 Syndrom des brennenden Mundes (engl. Burning mouth syndrome, BMS) 6.1.1 Syndrom des brennenden Mundes ohne somatosensorische Veränderungen

S143 6.1.2 Syndrom des brennenden Mundes mit somatosensorischen Veränderungen

S143 6.1.3 Wahrscheinliches Syndrom des brennenden Mundes

S143 6.2 Anhaltender idiopathischer Gesichtsschmerz (engl. Persistent idiopathic facial pain, PIFP)

S144 6.2.1 Anhaltender idiopathischer Gesichtsschmerz ohne somatosensorische Veränderungen

S144 6.2.2 Anhaltender idiopathischer Gesichtsschmerz mit somatosensorischen Veränderungen

S144 6.2.3 Wahrscheinlicher anhaltender idiopathischer Gesichtsschmerz

S144 6.3 Anhaltende idiopathische dentoalveoläre Schmerzen

S145 6.3.1 Anhaltende idiopathische dentoalveoläre Schmerzen ohne somatosensorische Veränderungen

S145 6.3.2 Anhaltende idiopathische dentoalveoläre Schmerzen mit somatosensorischen Veränderungen

S145 6.3.3 Wahrscheinliche anhaltende idiopathische dentoalveoläre Schmerzen

S145 6.4 Constant unilateral facial pain with additional attacks (CUFPA)

S146 Literatur

S146 7 Psychosoziale Beurteilung von Patienten mit orofazialen Schmerzen Einführung

S147 Ebenen der psychosozialen Beurteilung 
S147 Schmerz- und funktionsbezogene Konstrukte und Instrumente für orofaziale Schmerzen

Schmerzausmaß

Schmerzintensität und schmerzbedingte Einschränkung

Funktionelle Einschränkung

Zu Überbeanspruchung führende Verhaltensweisen

S148 Psychosoziale Konstrukte und Instrumente im Zusammenhang mit orofazialen Schmerzen

Depression und Angst

Somatoforme Störungen

Katastrophisieren

Angstvermeidung (Fear Avoidance)

S148 Schlussfolgerungen und Zukunftsperspektive

S149 Literatur

S150 Glossar zur ICOP

S151 Literatur

Hier steht eine Anzeige.

Springer 


\section{Vorwort}

Wir sind stolz darauf, diese erste Auflage der International Classification of Orofacial Pain (Internationalen Klassifikation orofazialer Schmerzen, ICOP) vorstellen zu können.

Bislang fehlt eine umfassende, international anerkannte Klassifikation, die sich mit orofazialen Schmerzen auseinandersetzt. Wenngleich das Gesicht anatomisch betrachtet eindeutig dem Kopf zuzuordnen ist, sind uns viel zu viele Fälle von Fehldiagnosen und dementsprechend unsachgemäßer Behandlung bekannt, die durch eine klare diagnostische Zuordnung womöglich zu vermeiden gewesen wären. Anatomische Abgrenzung und entsprechend getrennte medizinische Fachgebiete tragen das Ihre zum Problem bei. So definiert zum Beispiel die International Headache Society (IHS) Gesichtsschmerz als "Schmerz unterhalb der Orbitomeatallinie, oberhalb des Halses und vor der Ohrmuschel". Bei anderen Definitionen des Gesichtsschmerzes wird zusätzlich die Stirn einbezogen, während der Begriff, ,orofazialer Schmerz" zwangsläufig auch sämtliche Strukturen innerhalb der Mundhöhle umfasst. Gleichzeitig strahlen Kopfschmerzen oft in orofaziale Regionen aus und umgekehrt. "Kopfschmerzen“ wiederum können ausschließlich innerhalb der orofazialen Region lokalisiert sein und diagnostisch erhebliche Schwierigkeiten bereiten, während orofaziale Schmerzen, die in den Kopf ausstrahlen, komplexe klinische Phänotypen darstellen.

Dies waren einige Faktoren, die uns klar den Bedarf an einer Klassifikation orofazialer Schmerzen (OFPs) zeigten. Ein grundlegendes Prinzip dieser ersten Klassifikation ist, dass die Charakteristika der Schmerzstörungen unabhängig vom Ort, an dem diese auftreten (Kopf oder Gesicht), bei der neuen Konzeptualisierung und der Zusammenstellung der Diagnosekriterien richtungsweisend sein sollten.

Bei der Erstellung dieser ersten Auflage der ICOP waren wir uns bewusst, dass in der Internationalen Klassifikation von Kopfschmerzerkrankungen, 3. Auflage (ICHD-3) eine grundlegende Unterscheidung in primäre und sekundäre Kopfschmerzen stattfindet und verweisen unsere Leserinnen und Leser diesbezüglich hierauf. Darüber hinaus haben wir, um die ICOP zu einem nützlichen Hilfsmittel für Forscher und Kliniker zu machen, die gewohnt sind, die ICHD-3 anzuwenden, die hierarchische Struktur und den Klassifizierungsstil der ICHD-3 übernommen. Die Diagnostic Criteria for Temporomandibular Disorders (Diagnostische Kriterien für eine temporomandibuläre Dysfunktion, DC/TMD) sind eine bewährte und gängige Klassifikation, die auch regionale Myalgien und ${ }_{\text {,Schmer- }}$ zen im Kiefer, im Schläfenbereich, im Ohr und vor dem Ohr" einbezieht. Wir haben die DC/TMD-Kriterien übernommen und dabei lediglich die schmerzhafte temporomandibuläre Dysfunktion mit aufgenommen sowie die Darstellung dieser Kriterien an die der ICHD-3 angepasst. Insgesamt liegt die ICOP auch auf einer Linie mit den Kriterien für orofaziale Schmerzen und Kopfschmerzen der 11. überarbeiteten Fassung der International Classification of Diseases (ICD-11)/International Association for the Study of Pain (IASP).

Unser Ziel ist die Schaffung eines Werkzeugs, das die Erforschung und das klinische Management orofazialer Schmerzen fördert. Darüber hinaus sind wir zuversichtlich, dass das von uns gewählte methodische Vorgehen Fachleute, die sich bei ihrer Arbeit mit Kopfschmerzen, orofazialen Schmerzen, Augen-, Nasen-, Sinus- und Nackenschmerzen beschäftigen, näher zusammenbringen und zu einer aktiven Zusammenarbeit anregen wird.

Die Mitglieder des ICOP-Klassifikationskomitees repräsentieren die wichtigen Fachgesellschaften, die sich mit orofazialen Schmerzen und Kopfschmerz beschäftigen, und bilden eine wahrlich internationale Gruppe, was die Zukunft der ICOP stärkt. Der Weg zu dieser ersten Auflage begann 2016, als wir uns beim World Congress of the International Association for the Study of Pain (IASP) in Yokohama, Japan, erstmals trafen. Im Rahmen des eintägigen Meetings besprachen wir den Aufbau der ICOP und riefen Arbeitsgruppen ins Leben. Ein Folgetreffen fand 2017 an der Rutgers School of Dental Medicine in den USA statt, wo wir uns mit der Evidenz (beziehungsweise fehlenden Evidenz) für die Festlegung der einzelnen Entitäten auseinandersetzten, aus denen sich die heutige ICOP zusammensetzt. Heraus kam eine Klassifikation, die auf vielen Gebieten von einer starken Evidenz gestützt wird und in anderen Bereichen auf Expertenmeinungen beruht, die Impulse und Richtschnur für die weitere Forschungsarbeit gibt. Sie sieht zudem einen Erprobungszeitraum vor, der die Ermittlung und Korrektur von Fehlern erlaubt und breiten Raum für Beiträge von auf diesem Gebiet tätigen Forschern und Praktikern zur ICOP lässt.

Rafael Benoliel

Arne May

Peter Svensson

(Gesichtsschmerz-Klassifikationskomitee)

\section{Anleitung zum Gebrauch der ICOP}

Da sich die ICOP am Modell der ICHD-3 orientiert, ist auch die Anleitung zu ihrem Gebrauch ähnlich. Viele Benutzer der ICHD-3 werden die Benutzung der ICOP entsprechend einfach finden. Wie die ICHD-3 versteht sich auch dieses Dokument als Nachschlagewerk, insbesondere für die Forschung, aber auch für die klinische Diagnostik und das Management orofazialer Schmerzen (OFPs).

Die ICOP soll als Forschungs- und Diagnosekompendium dienen. Besonderen Nutzen hat sie bei unsicherer Diagnose oder wenn ein Behandler nicht weiß, dass ein solches klinisches Erscheinungsbild existiert. Wir empfehlen, dass Ärzte und Wissenschaftler sich mit dieser Klassifikation vertraut machen. Ferner greift die ICOP noch einmal das Bemühen der ICHD-3 und ICD-11/IASP auf, eine klare Terminologie festzulegen, die Eindeutigkeit in der Kommunikation und beim Austausch von Daten ermöglicht. Für wissenschaftliche Zwecke ist die Klassifikation unverzichtbar: Jeder Patient, der in ein Studienprojekt aufgenommen werden soll, sei es in eine Medikamentenstudie, eine pathophysiologische oder biochemische Untersuchung, muss eine Reihe der festgelegten diagnostischen Kriterien erfüllen.

Die Klassifikation ist hierarchisch gegliedert. Der Anwender kann dementsprechend selbst entscheiden, wie detailliert seine Diagnose ausfallen soll. Reicht die erste Stelle oder soll sie bis zur fünften, sechsten oder siebten heruntergebrochen werden? Die gewünschte Detailtiefe bei der diagnostischen Kodierung hängt eindeutig vom beabsichtigten Verwendungszweck ab. In der Praxis des Allgemeinmediziners werden in der Regel nur Diagnosen be- 
nötigt werden, die auf die 1. oder 2. Stelle exakt sind, während in speziellen Fachpraxen Diagnosen bis zur Ebene der 4. oder 5. Stelle (und gelegentlich der 6. oder 7.) angebracht sind.

Wir handeln primäre und sekundäre orofaziale Schmerzen innerhalb derselben Abschnitte ab. Hierin liegt ein Unterschied zur ICHD, doch handelt es sich nach unserer Meinung um eine effizientere Art, die verschiedenen orofazialen Schmerzen vorzustellen - teilweise deshalb, weil die Unterscheidung in primär und sekundär bei diesen sich überschneidenden Störungsbildern schwierig ist. Aus ähnlichen Gründen funktioniert die strenge Struktur der ICHD-Kriterien für sekundäre Störungen - wobei Kriterium A den Schmerz darlegt, Kriterium $B$ dessen mutmaßliche Ursache und Kriterium $C$ festschreibt, wie der Nachweis des ursächlichen Zusammenhangs von A mit B beschaffen sein muss - für orofaziale Schmerzen nicht. Vor allem variiert der zeitliche Zusammenhang zwischen dem Schmerzbeginn und dem Beginn oder Auftreten der vermuteten Ursache - der Eckpfeiler von Kriterium C in der ICHD bei orofazialen Schmerzen oft so stark, dass er keinen Beweiswert hat. Häufig beruht die Zuordnung auf der klinischen Plausibilität eines ursächlichen Zusammenhangs, dies jedoch immer vor dem Hintergrund der Berücksichtigung des letzten Kriteriums: „Nicht besser erklärt durch eine andere ICOP-Diagnose“ (siehe unten).

\section{Anwendungsprinzipien}

1. Erfüllt ein Patient die Kriterien für mehr als einen Typ, einen Subtyp oder eine Unterform von orofazialen Schmerzen und Kopfschmerzen, sind diese jeweils separat zu diagnostizieren und kodieren. So kann zum Beispiel ein stark betroffener Patient gleich mehrere Diagnosen erhalten, und es kann eine Mehrfachkodierung erfolgen: als 2.1.2.3.2 Chronische sehr häufige primäre myofaszial-orofaziale Schmerzen mit Schmerzübertragung und 3.1.4.1 Chronische persistierende primäre Kiefergelenksschmerzen ohne Schmerzübertragung sowie möglicherweise auch als 1.1 Migräne ohne Aura aus der ICHD-3.

2. Erhält ein Patient mehr als eine Diagnose, sollten die Diagnosen in der Reihenfolge ihrer Wichtigkeit für den Patienten aufgelistet werden, also abhängig davon, welche der Diagnosen aus der Sicht des Patienten mit dem größten Leidensdruck und der massivsten Beeinträchtigung verbunden ist.

3. Falls unklar ist, um welchen Typ von orofazialen Schmerzen es sich bei einem Patienten handelt, sollten zusätzlich zu den diagnostischen Kriterien weitere verfügbare Informationen einbezogen werden, um zu entscheiden, welche Diagnose die wahrscheinlichere ist. Dies kann den Verlauf der Schmerzerkrankung (wie und wann setzten die Schmerzen ein?), die Familienanamnese, die Wirkung von Medikamenten, den Menstruationszyklus, das Alter und Geschlecht sowie eine Reihe weiterer Merkmale umfassen.

4. Um eine bestimmte orofaziale Diagnose zu erhalten, muss der Patient in vielen Fällen eine bestimmte Mindestanzahl an Schmerzattacken dieser Art oder eine bestimmte Anzahl von Tagen mit diesen Schmerzen erleben. Diese Anzahl wird dann in den diagnostischen Kriterien für den betreffenden Typ oder Subtyp von orofazialen Schmerzen spezifiziert. Die Diagnose orofazialer Schmerzen muss eine Reihe weiterer Anforderun- gen erfüllen, die innerhalb der Kriterien separat unter A, B, C etc. dargelegt werden. Einige davon sind monothetisch: das heißt, sie drücken eine einzige Anforderung aus. Andere sind polythetisch und setzen beispielsweise voraus, dass zwei von vier aufgeführten Charakteristika vorliegen. Diese Struktur wurde von der ICHD-3 übernommen.

5. Die Häufigkeit des Auftretens orofazialer Schmerzen variiert stark, von nur einmal in 1-2 Jahren bis zu täglichen Schmerzen. Auch die Heftigkeit der Attacken variiert. Außer für myofasziale Schmerzen und Kiefergelenksschmerzen bietet die ICOP keine Möglichkeit, die Häufigkeit zu kodieren. Keine der diagnostischen Kriterien beinhalten eine routinemäßige Beurteilung des Schweregrads und der Häufigkeit, wir empfehlen jedoch eine Beurteilung und Spezifizierung der Häufigkeit und Stärke.

6. Das letzte Kriterium für fast jedes Krankheitsbild lautet „Nicht besser erklärt durch eine andere ICOP-Diagnose“ (mitunter auch „Nicht besser erklärt durch eine andere ICOP- oder ICHD-3-Diagnose"). Andere mögliche Diagnosen in Erwägung zu ziehen (die Differenzialdiagnose), ist ein routinemäßiger Bestandteil des klinischen Diagnoseverfahrens. Wenn ein orofazialer Schmerz die Kriterien für ein bestimmtes Krankheitsbild zu erfüllen scheint, erinnert dieses letzte Kriterium immer daran, andere Diagnosen in Betracht zu ziehen, die die orofazialen Schmerzen besser erklären könnten. Um dieses Ziel der Differenzialdiagnose zu erreichen, gilt es, beim klinisch-diagnostischen Prozess andere Krankheitsbilder jenseits des von der ICOP abgedeckten Rahmens zu berücksichtigen, eine detaillierte Schmerzanamnese zu erarbeiten und klinische Tests durchzuführen, die über die in den ICOP-Kriterien angesprochenen hinausgehen. Eine Schmerzübertragung von einer orofazialen Struktur zur anderen kommt außerordentlich häufig vor, dies alles jedoch ebenfalls zu klassifizieren, würde den Rahmen der ICOP sprengen.

Das Gebot, immer auch andere in Frage kommende Diagnosen in Betracht zu ziehen, gilt ähnlich grundlegend für die Forschung, welche erfordert, dass nur klar umrissene Fälle einbezogen werden. Unter diese Kategorie fällt allerdings nicht die gesamte Forschungsarbeit. Vor allem bei epidemiologischen Untersuchungen ist man vielleicht gar nicht in der Lage, das letztgenannte Kriterium anzuwenden (die Kopfschmerz-Epidemiologie war im Großen und Ganzen nicht imstande, das entsprechende Kriterium in der ICHD-3 anzuwenden). Untersuchungen, die dieses letzte Kriterium fallenlassen, sollten diese Tatsache zumindest in der Beschreibung ihres methodischen Vorgehens erwähnen.

7. Besteht bei Patienten der Verdacht auf mehr als eine Form von orofazialen Schmerzen, empfiehlt es sich sehr, dass diese ein diagnostisches Schmerztagebuch führen. Schmerztagebücher verbessern nachweislich nicht nur die exakte Diagnosestellung, sondern erlauben auch eine genauere Beurteilung des Medikamentenverbrauchs. Das Führen eines Schmerztagebuchs wird in der Regel für 1 Monat empfohlen. Während dieser Zeit werden für jede Schmerzepisode die wichtigen Charakteristika aufgezeichnet. Zusätzlich hilft das Schmerztagebuch dabei, die Häufigkeitsverteilung zwischen verschie- 
denen Typen, Subtypen oder Unterformen von orofazialen Schmerzen zu beurteilen. Zu guter Letzt ist die Verwendung des Tagebuchs ein wichtiges Hilfsmittel, um Patienten zu erklären, woran sie verschiedene orofaziale Schmerzereignissen voneinander unterscheiden können, wie sie ihren Arzneimittelkonsum im Blick behalten, Triggerfaktoren wahrnehmen und bei der Verlaufskontrolle eine zuverlässige Informationsquelle sein können.

\section{ICOP-Code Diagnose}

1

1.1

1.1.1

1.1.1.1

1.1.1.1.1

1.1.1.1.2

1.1.1.1.2.1

1.1.1.1.2.2

1.1.1.1.2.3

1.1.1.1.3

1.1.1.1.3.1

1.1.1.1.3.2

1.1.1.1.3.3

1.1.1.1.4

1.1.1.1.5

1.1.1.2

1.1.1.3

1.1.1.3.1

1.1.1.3.1.1

1.1.1.3.1.2
Orofaziale Schmerzen zurückzuführen auf Erkrankungen und Dysfunktionen dentoalveolärer und anatomisch benachbarter Strukturen

Zahnschmerzen

Schmerzen der Zahnpulpa

Schmerzen der Zahnpulpa zurückzuführen auf Überempfindlichkeit

Schmerzen der Zahnpulpa zurückzuführen auf einen Riss im Zahnschmelz

Schmerzen der Zahnpulpa zurückzuführen auf freiliegendes Dentin

Schmerzen der Zahnpulpa zurückzuführen auf Zahnabnutzung oder -abrasion

Schmerzen der Zahnpulpa zurückzuführen auf eine Fraktur mit hieraus resultierendem freiliegendem Dentin

Schmerzen der Zahnpulpa zurückzuführen auf einen Entwicklungsdefekt des Zahnhartgewebes

Schmerzen der Zahnpulpa zurückzuführen auf einen zahnärztlichen Eingriff

Schmerzen der Zahnpulpa zurückzuführen auf eine großfächige Entfernung von Dentinsubstanz

Schmerzen der Zahnpulpa zurückzuführen auf eine vorangegangene Zahnrestauration

Schmerzen der Zahnpulpa zurückzuführen auf okklusale Frühkontakte oder Interferenzen nach restaurativen zahnärztlichen Maßnahmen

Schmerzen der Zahnpulpa zurückzuführen auf eine zentrale Sensibilisierung

Schmerzen der Zahnpulpa zurückzuführen auf Überempfindlichkeit aufgrund einer anderen Ursache

Schmerzen der Zahnpulpa zurückzuführen auf freiliegende Zahnpulpa infolge eines Zahntraumas Schmerzen der Zahnpulpa zurückzuführen auf eine Pulpitis (Entzündung der Zahnpulpa)

Schmerzen der Zahnpulpa zurückzuführen auf eine reversible Pulpitis aufgrund einer Infektion des Dentins

Schmerzen der Zahnpulpa zurückzuführen auf eine reversible Pulpitis aufgrund einer Karies, die nicht die Pulpa erreicht

Schmerzen der Zahnpulpa zurückzuführen auf eine reversible Pulpitis aufgrund einer Fraktur im dentalen Hartgewebe mit freiliegendem Dentin
1.1.1.3.1.3

1.1.1.3.2

1.1.1.3.2.1

1.1.1.3.2.2

1.1.1.3.2.3

1.1.1.3.3

1.1.1.3.3.1

1.1.1.3.3.2

1.1.1.3.4

1.1.1.3.5

1.1.1.4

1.1 .2

1.1.2.1

1.1.2.1.1

1.1.2.1.1.1

1.1.2.1.1.2

1.1.2.1.1.3

1.1.2.1.1.4

1.1.2.1.2

1.1.2.1.2.2

1.1.2.1.2.2.1

1.1.2.1.2.2.2
Schmerzen der Zahnpulpa zurückzuführen auf eine reversible Pulpitis aufgrund eines Risses oder Sprungs im Zahn ohne Hinweis auf fehlende Zahnsubstanz

Schmerzen der Zahnpulpa zurückzuführen auf eine irreversible Pulpitis aufgrund einer Infektion des Dentins

Schmerzen der Zahnpulpa zurückzuführen auf eine irreversible Pulpitis aufgrund einer Karies, die bis zur Zahnpulpa reicht

Schmerzen der Zahnpulpa zurückzuführen auf eine irreversible Pulpitis aufgrund einer Fraktur dentaler Hartgewebe ohne Freilegung der Zahnpulpa

Schmerzen der Zahnpulpa zurückzuführen auf eine irreversible Pulpitis aufgrund eines Risses oder Sprungs im Zahn ohne Nachweis fehlender Zahnsubstanz

Schmerzen der Zahnpulpa zurückzuführen auf eine irreversible Pulpitis aufgrund einer Infektion der Zahnpulpa

Schmerzen der Zahnpulpa zurückzuführen auf eine irreversible Pulpitis aufgrund einer kariösen Freilegung und Infektion der Zahnpulpa

Schmerzen der Zahnpulpa zurückzuführen auf eine irreversible Pulpitis aufgrund einer Fraktur der dentalen Hartgewebe mit freiliegender Zahnpulpa

Schmerzen der Zahnpulpa zurückzuführen auf eine Pulpitis aufgrund einer externen zervikalen Wurzelresorption

Schmerzen der Zahnpulpa zurückzuführen auf eine Pulpitis aufgrund einer anderen Ursache

Schmerzen der Zahnpulpa zurückzuführen auf eine systemische Ursache

Parodontale Schmerzen

Parodontale Schmerzen zurückzuführen auf eine Parodontitis (parodontale Entzündung)

Parodontale Schmerzen zurückzuführen auf eine traumatisch bedingte parodontale Entzündung Parodontale Schmerzen zurückzuführen auf okklusale Frühkontakte oder Interferenzen

Postoperative parodontale Schmerzen

Parodontale Schmerzen zurückzuführen auf ein unfallbedingtes Zahntrauma

Parodontale Schmerzen zurückzuführen auf ein sonstiges Trauma oder eine sonstige Verletzung

Parodontale Schmerzen zurückzuführen auf eine apikale Parodontitis aufgrund einer endodontischen Erkrankung

Parodontale Schmerzen zurückzuführen auf eine Entzündung der Zahnpulpa

Parodontale Schmerzen zurückzuführen auf eine endodontische Infektion

Parodontale Schmerzen zurückzuführen auf eine intraradikuläre endodontische Infektion

Parodontale Schmerzen zurückzuführen auf eine extraradikuläre endodontische Infektion 
1.1.2.1.3

Parodontale Schmerzen zurückzuführen auf eine parodontale Erkrankung

1.1.2.1.3.1 Parodontale Schmerzen zurückzuführen auf eine chronische Parodontitis

1.1.2.1.3.2 Parodontale Schmerzen zurückzuführen auf eine aggressive Parodontitis

1.1.2.1.3.3 Parodontale Schmerzen zurückzuführen auf eine Parodontitis als Manifestation einer systemischen Erkrankung

1.1.2.1.3.3.1 Parodontale Schmerzen zurückzuführen auf eine hämatologische Erkrankung

1.1.2.1.3.3.2 Parodontale Schmerzen zurückzuführen auf eine genetische Störung

1.1.2.1.3.3.3 Parodontale Schmerzen zurückzuführen auf eine nicht näher spezifizierte systemische Erkrankung

1.1.2.1.3.4 Parodontale Schmerzen zurückzuführen auf eine nekrotisierende ulzerative Parodontitis (NUP)

1.1.2.1.3.5 Parodontale Schmerzen zurückzuführen auf einen parodontalen Abszess

1.1.2.1.4 Parodontale Schmerzen zurückzuführen auf eine apikale und marginale Parodontitis aufgrund einer Kombination von endodontischer Infektion und Parodontitis

1.1.2.1.5 Parodontale Schmerzen zurückzuführen auf eine infektiöse Periimplantitis

1.1.2.2 Parodontale Schmerzen zurückzuführen auf eine lokale nichtentzündliche Ursache

1.1.3 Gingivale Schmerzen

1.1.3.1 Gingivale Schmerzen zurückzuführen auf eine Gingivitis (Zahnfleischentzündung)

1.1.3.1.1 Gingivale Schmerzen zurückzuführen auf ein Trauma

1.1.3.1.2 Gingivale Schmerzen zurückzuführen auf eine Infektion

1.1.3.1.2.1 Gingivale Schmerzen zurückzuführen auf eine bakterielle Infektion

1.1.3.1.2.2 Gingivale Schmerzen zurückzuführen auf eine Virusinfektion

1.1.3.1.2.3 Gingivale Schmerzen zurückzuführen auf eine Pilzinfektion

1.1.3.1.3 Gingivale Schmerzen zurückzuführen auf eine Autoimmunreaktion

1.1.3.1.4 Gingivale Schmerzen zurückzuführen auf eine Überempfindlichkeit oder allergische Reaktion

1.1.3.1.5 Gingivale Schmerzen zurückzuführen auf eine Zahnfleischentzündung aufgrund einer anderen Ursache

1.1.3.2 Gingivale Schmerzen zurückzuführen auf eine maligne Läsion

1.2 Schmerzen der Mundschleimhaut, der Speicheldrüse und des Kieferknochens

1.2.1 Schmerzen in der Mundschleimhaut

1.2.1.1 Schmerzen in der Mundschleimhaut zurückzuführen auf eine Mundschleimhautentzündung

1.2.1.1.1 Schmerzen in der Mundschleimhaut zurückzuführen auf ein Trauma oder eine Verletzung
1.2.1.1.1.1 Schmerzen in der Mundschleimhaut zurückzuführen auf ein nichtiatrogenes Trauma oder eine nichtiatrogene Verletzung

1.2.1.1.1.2 Schmerzen in der Mundschleimhaut zurückzuführen auf eine chirurgische oder sonstige lokale iatrogene Verletzung

1.2.1.1.1.3 Schmerzen in der Mundschleimhaut zurückzuführen auf eine Bestrahlung oder Chemotherapie

1.2.1.1.2 Schmerzen in der Mundschleimhaut zurückzuführen auf eine Infektion

1.2.1.1.2.1 Schmerzen in der Mundschleimhaut zurückzuführen auf eine bakterielle Infektion

1.2.1.1.2.2 Schmerzen in der Mundschleimhaut zurückzuführen auf eine Virusinfektion

1.2.1.1.2.3 Schmerzen in der Mundschleimhaut zurückzuführen auf eine Pilzinfektion

1.2.1.1.3 Schmerzen in der Mundschleimhaut zurückzuführen auf eine Autoimmunreaktion

1.2.1.1.4 Schmerzen in der Mundschleimhaut zurückzuführen auf eine Überempfindlichkeit oder allergische Reaktion

1.2.1.1.5 Schmerzen in der Mundschleimhaut zurückzuführen auf eine Entzündung der Mundschleimhaut anderen Ursprungs

1.2.1.2 Schmerzen in der Mundschleimhaut zurückzuführen auf eine maligne Läsion

1.2.2 Schmerzen der Speicheldrüse

1.2.2.1 Schmerzen der Speicheldrüse zurückzuführen auf eine obstruktive Ursache

1.2.2.2 Schmerzen der Speicheldrüse zurückzuführen auf eine Infektion

1.2.2.2.1 Schmerzen der Speicheldrüse zurückzuführen auf eine bakterielle Infektion

1.2.2.2.2 Schmerzen der Speicheldrüse zurückzuführen auf eine Virusinfektion

1.2.2.3 Schmerzen der Speicheldrüse zurückzuführen auf eine chronische rezidivierende Parotitis des Kindesalters

1.2.2.4 Schmerzen der Speicheldrüse zurückzuführen auf eine immunologisch vermittelte Störung

1.2.2.5 Schmerzen der Speicheldrüse zurückzuführen auf eine andere Ursache

1.2.3

1.2.3.1

1.2.3.2

1.2.3.2.1

1.2.3.2.2

1.2.3.2.3

1.2.3.3 Schmerzen im Kieferknochen

Schmerzen im Kieferknochen zurückzuführen auf ein Trauma oder eine Verletzung

Schmerzen im Kieferknochen zurückzuführen auf eine Infektion

Schmerzen im Kieferknochen zurückzuführen auf eine bakterielle Infektion

Schmerzen im Kieferknochen zurückzuführen auf eine Virusinfektion

Schmerzen im Kieferknochen zurückzuführen auf eine Pilzinfektion

Schmerzen im Kieferknochen zurückzuführen auf eine lokale benigne Läsion 
1.2.3.4 Schmerzen im Kieferknochen zurückzuführen auf eine maligne Läsion

1.2.3.4.1 Schmerzen im Kieferknochen zurückzuführen auf einen lokalen Tumor

1.2.3.4.2 Schmerzen im Kieferknochen zurückzuführen auf einen Ferntumor

1.2.3.5 Schmerzen im Kieferknochen zurückzuführen auf eine Therapie

1.2.3.6 Schmerzen im Kieferknochen zurückzuführen auf eine systemische Erkrankung

2

2.1

2.1.1

2.1.2

2.1.2.1

2.1.2.2

2.1.2.2.1

2.1.2.3.1

2.1.2.3.2

2.2

3

3.1

3.1.1

3.1.2

3.1.2.1

3.1.2.2

3.1.2.2.1

3.1.2.2.2

3.1.2.3

3.1.2.3.1

3.1.2.3.2

3.2
Myofaszial-orofaziale Schmerzen

Primäre myofaszial-orofaziale Schmerzen

Akute primäre myofaszial-orofaziale Schmerzen

Chronische primäre myofaszial-orofaziale Schmer-

zen

Chronischer primärer myofaszial-orofazialer Gelegenheitsschmerz

Chronische häufige primäre myofaszial-orofaziale Schmerzen

Chronische häufige primäre myofaszial-orofaziale

Schmerzen ohne Schmerzübertragung

Chronische häufige primäre myofaszial-orofaziale

Schmerzen mit Schmerzübertragung

Chronische sehr häufige primäre myofaszial-orofa-

ziale Schmerzen

Chronische sehr häufige primäre myofaszial-orofaziale Schmerzen ohne Schmerzübertragung

Chronische sehr häufige primäre myofaszial-orofa-

ziale Schmerzen mit Schmerzübertragung

Sekundäre myofaszial-orofaziale Schmerzen

Myofaszial-orofaziale Schmerzen zurückzuführen

auf eine Tendinitis

Myofaszial-orofaziale Schmerzen zurückzuführen

auf eine Myositis

Myofaszial-orofaziale Schmerzen zurückzuführen

auf einen Muskelspasmus

Kiefergelenksschmerzen

Primäre Kiefergelenksschmerzen

Akute primäre Kiefergelenksschmerzen

Chronische primäre Kiefergelenksschmerzen

Chronische primäre gelegentliche Kiefergelenksschmerzen

Chronische häufige primäre Kiefergelenksschmerzen

Chronische häufige primäre Kiefergelenksschmerzen ohne Schmerzübertragung

Chronische häufige primäre Kiefergelenksschmerzen mit Schmerzübertragung

Chronische sehr häufige primäre Kiefergelenksschmerzen

Chronische sehr häufige primäre Kiefergelenks-

schmerzen ohne Schmerzübertragung

Chronische sehr häufige primäre Kiefergelenks-

schmerzen mit Schmerzübertragung

Sekundäre Kiefergelenksschmerzen
3.2.1

3.2.1.1

3.2.1.2

3.2 .2

3.2.2.1

3.2.2.1.1

3.2.2.2

3.2 .3

3.2.4

4

4.1

4.1.1

4.1.1.1

4.1.1.1.1

4.1.1.1.2

4.1.1.2

4.1.1.2.1

4.1.1.2.2

4.1.1.2.3

4.1.1.3

4.1.1.3.1

4.1.1.3.2

4.1.2

4.1.2.1

4.1.2.2

4.1.2.3

4.1.2.3.1

4.1.2.4

4.1.2.4.1

4.1.2.5

4.2
Kiefergelenksschmerzen zurückzuführen auf eine Arthritis

Kiefergelenksschmerzen zurückzuführen auf eine nichtsystemische Arthritis

Kiefergelenksschmerzen zurückzuführen auf eine systemische Arthritis

Kiefergelenksschmerzen zurückzuführen auf eine Diskusverlagerung

Kiefergelenksschmerzen zurückzuführen auf eine Diskusverlagerung mit Reduktion

Kiefergelenksschmerzen zurückzuführen auf eine Diskusverlagerung mit Reduktion, mit intermittierender Kieferklemme

Kiefergelenksschmerzen zurückzuführen auf eine Diskusverlagerung ohne Reduktion

Kiefergelenksschmerzen zurückzuführen auf eine degenerative Gelenkerkrankung

Kiefergelenksschmerzen zurückzuführen auf eine Subluxation

Orofaziale Schmerzen zurückzuführen auf eine Läsion oder Erkrankung der Hirnnerven

Schmerzen zurückzuführen auf eine Läsion oder Erkrankung des N. trigeminus

Trigeminusneuralgie

Klassische Trigeminusneuralgie

Klassische Trigeminusneuralgie, rein paroxysmal

Klassische Trigeminusneuralgie mit Dauerschmerz Sekundäre Trigeminusneuralgie

Trigeminusneuralgie zurückzuführen auf multiple Sklerose

Trigeminusneuralgie zurückzuführen auf eine raumfordernde Läsion

Trigeminusneuralgie zurückzuführen auf eine andere Ursache

Idiopathische Trigeminusneuralgie

Idiopathische Trigeminusneuralgie, rein paroxysmal

Idiopathische Trigeminusneuralgie mit Dauerschmerz

Andere neuropathische Schmerzen im Innervationsgebiet des N. trigeminus

Schmerzhafte Trigeminusneuropathie zurückzuführen auf Herpes Zoster

Postherpetische Trigeminusneuralgie

Posttraumatische schmerzhafte Trigeminusneuropathie

Wahrscheinliche posttraumatische schmerzhafte Trigeminusneuropathie

Schmerzhafte Trigeminusneuropathie zurückzuführen auf eine andere Erkrankung

Wahrscheinliche schmerzhafte Trigeminusneuropathie zurückzuführen auf eine andere Erkrankung Idiopathische schmerzhafte Trigeminusneuropathie

Schmerzen zurückzuführen auf eine Läsion oder Erkrankung des N. glossopharyngeus 
4.2.1

4.2.1.1

4.2.1.2

4.2.1.3

4.2.2

4.2.2.1

4.2.2.2

5

5.1

5.1.1

5.1.2

5.2

5.3

5.3.1

5.3.1.1

5.3.1.2

5.3.2

5.3.2.1

5.3.2.2

5.3.3

5.3.3.1

5.3.3.2

5.3.4

5.4

5.4.1

5.4 .2

6.

6.1

6.1.1

6.1 .2

6.1.3

6.2

6.2.1

6.2 .2

6.2 .3

6.3

6.3.1
Glossopharyngeusneuralgie

Klassische Glossopharyngeusneuralgie

Sekundäre Glossopharyngeusneuralgie

Idiopathische Glossopharyngeusneuralgie

Neuropathische Schmerzen im Innervationsgebiet

des N. glossopharyngeus

Schmerzhafte Glossopharyngeusneuropathie zu-

rückzuführen auf eine bekannte Ursache

Idiopathische schmerzhafte Glossopharyngeus-

neuropathie

Orofaziale Schmerzen mit Ähnlichkeit zu primären

Kopfschmerzerkrankungen

Orofaziale Migräne

Episodische orofaziale Migräne

Chronische orofaziale Migräne

Orofaziale Schmerzen vom Spannungstyp

Trigeminoautonome orofaziale Schmerzen

Orofaziale Clusterattacken

Episodische orofaziale Clusterattacken

Chronische orofaziale Clusterattacken

Paroxysmale hemifaziale Schmerzen

Episodische paroxysmale hemifaziale Schmerzen

Chronische paroxysmale hemifaziale Schmerzen

Short-lasting unilateral neuralgiform facial pain attacks (SUNFA) mit kranialen autonomen Sympto-

men

Episodische SUNFA

Chronische SUNFA

Hemifazialer Dauerschmerz mit autonomen Symptomen

Neurovaskuläre orofaziale Schmerzen

Kurz anhaltende neurovaskuläre orofaziale Schmer-

zen

Lang anhaltende neurovaskuläre orofaziale Schmer-

zen

Idiopathische orofaziale Schmerzen

Syndrom des brennenden Mundes (engl. Burning mouth syndrome, BMS)

Syndrom des brennenden Mundes ohne somatosensorische Veränderungen

Syndrom des brennenden Mundes mit somatosensorischen Veränderungen

Wahrscheinliches Syndrom des brennenden Mundes

Anhaltender idiopathischer Gesichtsschmerz (engl. Persistent idiopathic facial pain, PIFP)

Anhaltender idiopathischer Gesichtsschmerz ohne somatosensorische Veränderungen

Anhaltender idiopathischer Gesichtsschmerz mit somatosensorischen Veränderungen

Wahrscheinlicher anhaltender idiopathischer Gesichtsschmerz

Anhaltende idiopathische dentoalveoläre Schmer-

zen

Anhaltende idiopathische dentoalveoläre Schmer-

zen ohne somatosensorische Veränderungen
6.3 .2

Anhaltende idiopathische dentoalveoläre Schmerzen mit somatosensorischen Veränderungen

6.3.3 Wahrscheinliche anhaltende idiopathische dentoalveoläre Schmerzen

6.4 Constant unilateral facial pain with additional attacks (CUFPA)

Psychosoziale Beurteilung von Patienten mit orofazialen Schmerzen 


\section{Klassifikation, mit Diagnosekriterien}

\section{Orofaziale Schmerzen zurückzuführen auf Erkrankungen und Dysfunktionen dentoalveolärer und anatomisch benachbarter Strukturen}

Beschreibung: Orofaziale Schmerzen infolge von Erkrankungen, Traumata oder Funktionsstörungen der Zahnpulpa, des Zahnbetts, der Gingiva, der Mundschleimhaut, der Speicheldrüsen oder des Kieferknochengewebes oder als Reaktion einer regulär funktionierenden Pulpa, die die Gefahr einer Zahnschädigung anzeigt.

Allgemeine Kommentare: Schmerzen, die auf dentoalveoläre und mit diesem Bereich verbundene Strukturen zurückgehen, sind der häufigste Grund für orofaziale Schmerzen. Im Allgemeinen liegt ein nozizeptiver oder inflammatorischer Schmerz vor. Es handelt sich generell um akute Schmerzen, die nicht länger als 3 Monate bestehen. Bei adäquater Behandlung der Grunderkrankung kommt es nicht zu einem Persistieren der Schmerzen über einen längeren Zeitraum. Der Schmerz kann prinzipiell anhaltend, wiederkehrend oder sporadisch sein. In vielen Fällen verursacht der natürliche Verlauf der Grunderkrankung Schwankungen im Hinblick auf sämtliche Symptome, einschließlich des Schmerzes, weshalb diese Art von Schmerz mitunter als episodisch beschrieben wird (weniger als 15 Tage im Monat auftretend, egal ob für mehr oder weniger als 3 Monate vorhanden). Liegt ein Schmerz mehr als 3 Monate vor und tritt an mindestens 15 Tagen im Monat auf, gilt dieser als chronisch.

Da Schmerzen in Verbindung mit dentoalveolären und anatomisch benachbarten Strukturen in erster Linie Symptom einer Erkrankung sind, mag auch eine Kategorisierung relevant sein, die den Behandlungsaspekt einbezieht. Bleibt die schmerzinduzierende Grundstörung völlig unbehandelt, bestehen akute Schmerzen in der Regel fort und werden schließlich chronisch. Das Gleiche gilt, wenn die Grunderkrankung erfolglos oder unzureichend behandelt wird, da die Störung (etwa eine lokale Infektion, ein Tumor oder eine systemische Erkrankung) und der begleitende Schmerz ebenfalls länger als 3 Monate bestehen können.

Im Allgemeinen ist die Unterscheidung zwischen akuten und chronischen Schmerzen wichtig, da chronische Schmerzen oft eine andere Art von Schmerzmanagement erfordern und ihre Prognose weniger günstig ausfällt. Es ist jedoch nicht bekannt, ob die akute, die episodische und die chronische Form von dentoalveolären Schmerzen (und anderer hier angesprochener Schmerzarten) sich außer mit Blick auf ihre Dauer in irgendeinem klinisch bedeutsamen Aspekt unterscheiden. Mangels verfügbarer Daten, die hinsichtlich der Behandlung oder Prognose für die Relevanz einer solchen Unterscheidung sprechen würden, wird auf die Abgrenzung akuter von chronischen Schmerzen in diesem Abschnitt der ICOP nicht eingegangen. Für Studien, die zum Beispiel einen Vergleich zwischen dentoalveolären Schmerzen von kurzer und solchen von langer Dauer anstreben, empfiehlt sich die in Übereinstimmung mit der IASP/ICD-11 und ICHD-3 oben beschriebene Unterscheidung zwischen akuten, episodischen und chronischen Schmerzen.

Sollten sich zukünftig in dieser Beziehung Hinweise auf wichtige Unterschiede zeigen, ist die Entscheidung, Schmerzzustände in diesem Abschnitt nicht nach Dauer zu unterscheiden, zu überdenken.

\subsection{Zahnschmerzen}

Beschreibung: Schmerzen infolge von Läsionen oder Störungen, die einen Zahn oder mehrere Zähne und/oder ihre unmittelbare Umgebung und den Zahnhalteapparat betreffen: Pulpa, Parodontium und Gingiva.

\subsubsection{Schmerzen der Zahnpulpa}

Beschreibung: Schmerzen infolge einer Läsion oder Störung, die die Zahnpulpa betrifft.

\section{Diagnostische Kriterien:}

A. Jeder Zahnschmerz, der Kriterium C erfüllt

B. Klinische Befunde, Laborwerte, bildgebende Verfahren und/ oder die Anamnese belegen eine Läsion, eine Erkrankung oder ein Trauma' ${ }^{1}$ durch welche bekanntermaßen Schmerzen der Pulpa hervorrufen werden können

C. Ein ursächlicher Zusammenhang lässt sich durch beide der folgenden Kriterien nachweisen:

1. Die Lokalisation des Schmerzes entspricht der Stelle/den Stellen der Läsion, der Erkrankung oder des Traumas ${ }^{2}$

2. Eines oder beides von Folgendem trifft zu:

a) Der Schmerz entstand in zeitlichem Zusammenhang mit dem Eintritt der Läsion bzw. dem Beginn der Erkrankung oder des Traumas oder führte zu deren/dessen Entdeckung

b) Der Schmerz verschlimmert sich durch Einwirkung eines physischen Reizes ${ }^{3}$ auf den betroffenen Zahn

D. Nicht besser erklärt durch eine andere ICOP-Diagnose

\section{Anmerkungen:}

1. Die Läsion, die Erkrankung oder das Trauma wird bei jeder Unterform spezifiziert.

2. Der Schmerz kann sich auch in andere ipsilaterale orofaziale Regionen ausbreiten und/oder ausstrahlen.

3. Der Reiz kann mechanischer, thermischer oder chemischer Art sein, wie bei manchen Unterformen spezifiziert.

Kommentar: 1.1.1 Schmerzen der Zahnpulpa können mit jeder Art von Verletzung oder Erkrankung der Pulpa einhergehen. Der Schmerz ist vorwiegend entzündlicher Natur und tritt in Folge äuBerer oder innerer Ereignisse auf.

\subsubsection{Schmerzen der Zahnpulpa zurückzuführen aufüberempfind- lichkeit}

Beschreibung: Schmerzen der Zahnpulpa aufgrund einer Überempfindlichkeit in einer ansonsten klinisch unauffälligen Pulpa.

\section{Diagnostische Kriterien:}

A. Zahnschmerz, der die Kriterien für 1.1.1 Schmerzen der Zahnpulpa sowie Kriterium B und $C$ erfüllt

B. Der Schmerz weist alle der folgenden Charakteristika auf:

1. Hervorgerufen durch äußere Reize ${ }^{1}$

2. Lässt innerhalb weniger Sekunden nach

3. Eines oder beides von Folgendem trifft zu:

a) Eine stechende, tiefe Schmerzempindung

b) Schlecht zu lokalisieren ${ }^{2}$ 
C. Ein ursächlicher Zusammenhang ist aufgrund einer anatomischen, funktionellen und/oder zeitlichen Verbindung plausibel $^{3}$

D. Nicht besser erklärt durch eine andere ICOP-Diagnose

\section{Anmerkungen:}

1. Zu den äußerlichen Stimuli, die Schmerzen hervorrufen können, gehören heiß, kalt und süß.

2. Oft nur auf ein ungefähres Gebiet zwei oder drei Nachbarzähne neben dem betroffenen Zahn beschränkt. Gelegentlich kann der Patient nicht ausmachen, ob der Schmerz vom Unter- oder Oberkiefer ausgeht.

3. Dieses Kriterium setzt sich auch bis zu allen Unterformen hinab fort.

1.1.1.1.1 Schmerzen der Zahnpulpa zurückzuführen auf einen Riss im Zahnschmelz

\section{Diagnostische Kriterien:}

A. Zahnschmerz, der die Kriterien für 1.1.1.1 Schmerzen der Zahnpulpa zurückzuführen aufüberempfindlichkeit sowie Kriterium C erfüllt

B. Ein Riss oder eine unvollständige Fraktur des betroffenen Zahns unter Einbezug des Zahnschmelzes wurde durch visuelle Identifizierung' der Bruchlinie(n) diagnostiziert

C. Mindestens eines von Folgendem trifft zu:

1. Stechender Schmerz beim Zubeißen

2. Schmerz bei Lösen des okklusalen Beißdrucks oder bei äuBerer Kraftanwendung

3. Kälteüberempfindlichkeit

D. Nicht besser erklärt durch eine andere ICOP-Diagnose

\section{Anmerkung:}

1. Bei Bedarf kann die Diagnostik durch Vergrößerungshilfen, starke Ausleuchtung und/oder Sichtbarmachung mit Farbstoff unterstützt werden.

1.1.1.1.2 Schmerzen der Zahnpulpa zurückzuführen auffreiliegendes Dentin

\section{Diagnostische Kriterien:}

A. Zahnschmerz' ${ }^{1}$, der die Kriterien für 1.1.1.1 Schmerzen der Zahnpulpa zurückzuführen auf Überempfindlichkeit erfüllt

B. Die Dentinoberfläche des betroffenen Zahns liegt frei

C. Nicht besser erklärt durch eine andere ICOP-Diagnose

\section{Anmerkung:}

1. Der Schmerz lässt sich in der Regel durch Kratzen am freiliegenden Dentin mit einer Sonde oder durch Druckluft reproduzieren.

1.1.1.1.2.1 Schmerzen der Zahnpulpa zurückzuführen auf Zahnabnutzung oder -abrasion

\section{Diagnostische Kriterien:}

A. Zahnschmerz, der die Kriterien für 1.1.1.1.2 Schmerzen der Zahnpulpa zurückzuführen auf freiliegendes Dentin erfüllt

B. Klinischer Nachweis von Abnutzung oder Verschleiß des betroffenen Zahns'
C. Nicht besser erklärt durch eine andere ICOP-Diagnose

\section{Anmerkung:}

1. Glatte, ebene Oberflächen, deren Kontur nicht der natürlichen Form der anatomischen Zahnkrone entspricht.

1.1.1.1.2.2 Schmerzen der Zahnpulpa zurückzuführen auf eine Fraktur mit hieraus resultierendem freiliegendem Dentin

Diagnostische Kriterien:

A. Zahnschmerz, der die Kriterien für 1.1.1.1.2 Schmerzen der Zahnpulpa zurückzuführen auf freiliegendes Dentin erfüllt

B. Es wurde eine Fraktur des betroffenen Zahns unter Beteiligung des Zahnschmelzes, Wurzelzements, Dentins oder einer Kombination hiervon diagnostiziert ${ }^{1}$

C. Nicht besser erklärt durch eine andere ICOP-Diagnose

\section{Anmerkung:}

1. Die Diagnose stützt sich auf klinische und/oder radiologische Beobachtungen.

1.1.1.1.2.3 Schmerzen der Zahnpulpa zurückzuführen auf einen Entwicklungsdefekt des Zahnhartgewebes

\section{Diagnostische Kriterien:}

A. Zahnschmerz, der die Kriterien für 1.1.1.1.2 Schmerzen der Zahnpulpa zurückzuführen auf freiliegendes Dentin erfüllt

B. Es wurde eine Entwicklungsstörung des betroffenen Zahns unter Beteiligung des Zahnschmelzes, Wurzelzements und/ oder Dentins diagnostiziert, die bekanntermaßen Schmerzen der Zahnpulpa' auslösen kann

C. Nicht besser erklärt durch eine andere ICOP-Diagnose

\section{Anmerkung:}

1. Zu den Entwicklungsstörungen dieser Art gehören eine lokale Hypomineralisation oder Hypomaturation des Zahnschmelzes, Amelogenesis imperfecta, Dentinogenesis imperfecta sowie eine Vielzahl sonstiger, seltenerer Defekte.

1.1.1.1.3 Schmerzen der Zahnpulpa zurückzuführen auf einen zahnärztlichen Eingriff

\section{Diagnostische Kriterien:}

A. Zahnschmerz, der die Kriterien für 1.1.1.1 Schmerzen der Zahnpulpa zurückzuführen auf Überempfindlichkeit erfüllt.

B. Es erfolgte kurz zuvor' eine Behandlung des betroffenen Zahns

C. Nicht besser erklärt durch eine andere ICOP-Diagnose

\section{Anmerkung:}

1. Der Schmerzeintritt erfolgt in der Regel Stunden bis Tage nach dem zahnärztlichen Eingriff.

1.1.1.1.3.1 Schmerzen der Zahnpulpa zurückzuführen auf eine großflächige Entfernung von Dentinsubstanz

\section{Diagnostische Kriterien:}

A. Zahnschmerz, der die Kriterien für 1.1.1.1.3 Schmerzen der Zahnpulpa zurückzuführen auf einen zahnärztlichen Eingriff erfüllt 
B. Kurz zuvor ${ }^{1}$ ist es zu einer Entfernung von Dentin an dem betroffenen Zahn gekommen, worauf eines oder beides von Folgendem zutrifft:

1. Tief (d. h. unmittelbare Nähe zur Pulpa)

2. Breit (d.h. großfächige Öffnung von Dentintubuli)

C. Nicht besser erklärt durch eine andere ICOP-Diagnose

\section{Anmerkung:}

1. Der Schmerzeintritt erfolgt in der Regel Stunden bis Tage nach dem zahnärztlichen Eingriff.

1.1.1.1.3.2 Schmerzen der Zahnpulpa zurückzuführen auf eine vorangegangene Zahnrestauration

\section{Diagnostische Kriterien:}

A. Zahnschmerz, der die Kriterien für 1.1.1.1.3 Schmerzen der Zahnpulpa zurückzuführen auf einen zahnärztlichen Eingriff erfüllt

B. Kurz zuvor ${ }^{1}$ erfolgte direkte oder indirekte Restauration am betroffenen Zahn

C. Nicht besser erklärt durch eine andere ICOP-Diagnose

\section{Anmerkung:}

1. Der Schmerzeintritt erfolgt in der Regel Stunden bis Tage nach dem zahnärztlichen Eingriff.

1.1.1.1.3.3 Schmerzen der Zahnpulpa zurückzuführen auf okklusale Frühkontakte oder Interferenzen nach restaurativen zahnärztlichen Maßnahmen

\section{Diagnostische Kriterien:}

A. Zahnschmerz, der die Kriterien für 1.1.1.1.3 Schmerzen der Zahnpulpa zurückzuführen auf einen zahnärztlichen Eingriff erfüllt

B. Eine restaurative Maßnahme ${ }^{1}$ hat beim betroffenen Zahn einen okklusalen Frühkontakt und/oder eine okklusale Interferenz verursacht

C. Nicht besser erklärt durch eine andere ICOP-Diagnose

\section{Anmerkung:}

1. Restaurative Maßnahmen umfassen provisorische Versorgungen, Zahnrestaurationen und prothetischen Zahnersatz.

1.1.1.1.4 Schmerzen der Zahnpulpa zurückzuführen auf eine zentrale Sensibilisierung

\section{Diagnostische Kriterien:}

A. Zahnschmerz an einem oder mehreren Zähnen, der die Kriterien für 1.1.1.1 Schmerzen der Zahnpulpa zurückzuführen auf Überempfindlichkeit und Kriterium C und D erfüllt

B. Es sind beide der folgenden Kriterien erfüllt:

1. Diagnose einer weiteren orofazialen, auf den Nacken bezogenen oder generalisierten Schmerzstörung

2. Zeichen einer zentralen Sensibilisierung ${ }^{1}$ liegen vor

C. Es ist mindestens eines der beiden folgenden Kriterien erfüllt:

1. Der Schmerz tritt spontan auf

2. Der Schmerz tritt im Verband mit dem anderen Schmerz auf und/oder schwankt mit diesem
D. Durch örtliche Betäubung oder peripher wirksame Analgetika nicht durchgängig zu lindern

E. Nicht besser erklärt durch eine andere ICOP-Diagnose

\section{Anmerkung:}

1. Zu den Zeichen gehören das Phänomen der Schmerzübertragung, die temporale Schmerzsummation und die Allodynie.

Kommentar: 1.1.1.1.4 Schmerzen der Zahnpulpa zurückzuführen auf eine zentrale Sensibilisierung können an mehreren Zähnen gleichzeitig auftreten oder aber an einem Zahn beginnen und dann auch andere Zähne erfassen. Es kann sich um Dauerschmerzen oder rezidivierende Schmerzen handeln, sie können über lange Zeiträume bestehen; oft sind die Schmerzen chronisch.

Die Schmerzsymptome können von einer Dentin-Überempfindlichkeit bis zu anhaltenden Schmerzen ähnlich wie bei einer Pulpitis reichen und treten oft in Begleitung autonomer Zeichen auf (siehe 5.1 Orofaziale Migräne).

Eine erfolgreiche Behandlung des begleitenden Schmerzes/der sonstigen Schmerzen und hiermit zusammenhängenden psychischen Symptome führt oft zu einer Reduzierung der Zahnschmerzen. Eine zahnärztliche Desensibilisierungsbehandlung kann in einigen Fällen zu einer Schmerzlinderung oder Schmerzbeseitigung führen.

1.1.1.1.5 Schmerzen der Zahnpulpa zurückzuführen aufüberempfindlichkeit aufgrund einer anderen Ursache

\section{Diagnostische Kriterien:}

A. Zahnschmerz an einem oder mehreren Zähnen, der die Kriterien für 1.1.1.1 Schmerzen der Zahnpulpa zurückzuführen auf Überempfindlichkeit sowie Kriterium C erfüllt

B. Es wurde eine Störung diagnostiziert, die erfahrungsgemäß Schmerzen der Zahnpulpa auslösen kann, jedoch eine andere Art, als unter 1.1.1.1.1 bis 1.1.1.1.4 beschrieben

C. Ein ursächlicher Zusammenhang ist aufgrund der anatomischen, funktionellen und/oder zeitlichen Verbindung plausibel

D. Nicht besser erklärt durch eine andere ICOP-Diagnose

\subsubsection{Schmerzen der Zahnpulpa zurückzuführen auf freiliegende} Zahnpulpa infolge eines Zahntraumas

\section{Diagnostische Kriterien:}

A. Zahnschmerz, der die Kriterien für 1.1.1 Schmerzen der Zahnpulpa sowie Kriterium C erfüllt

B. Das Frontzahntrauma führte zu einem oder mehreren der folgenden Punkte und hat dabei vitales Pulpagewebe im betroffenen Zahn freigelegt:

1. Fraktur unter Beteiligung von Zahnschmelz, Dentin und Pulpa (komplizierte Kronenfraktur)

2. Fraktur unter Beteiligung des Wurzelzements, des Dentins und der Pulpa (komplizierte Wurzelfraktur)

3. Fraktur unter Beteiligung von Zahnschmelz, Wurzelzement, Dentin und Pulpa (komplizierte Kronen-Wurzel-Fraktur)

C. Schmerzentwicklung innerhalb von Minuten bis Stunden nach dem Trauma

D. Nicht besser erklärt durch eine andere ICOP-Diagnose 
Kommentar: 1.1.1.2Schmerzen der Zahnpulpa zurückzuführen auf freiliegende Zahnpulpa infolge eines Zahntraumas sind leicht bis mittelstark. Charakteristischerweise verschlimmern sich diese durch Einwirkung von Luft, Flüssigkeiten oder Druck auf das freiliegende Pulpagewebe, legen sich aber, wenn der Reiz aufhört. Im Zeitraum unmittelbar nach Eintritt des Traumas fehlen jedoch Temperaturempfindlichkeit, Spontanschmerz oder ausstrahlende Schmerzen, da diese Symptome in der Regel erst später auftreten und mit Entzündungsprozessen zusammenhängen.

\subsubsection{Schmerzen der Zahnpulpa zurückzuführen auf eine Pulpitis} (Entzündung der Zahnpulpa)

\section{Diagnostische Kriterien:}
A. Zahnschmerz, der die Kriterien für 1.1.1 Schmerzen der Zahn- pulpa sowie Kriterium C erfüllt
B. Es wurde eine Pulpitis am betroffenen Zahn ${ }^{1}$ diagnostiziert
C. Ein ursächlicher Zusammenhang ist aufgrund einer anatomi- schen, funktionellen und/oder zeitlichen Verbindung plausi- bel $^{2}$
D. Nicht besser erklärt durch eine andere ICOP-Diagnose

\section{Anmerkungen:}

1. Eine Pulpitis kann traumatisch oder infektiös bedingt sein, wie bei den einzelnen Unterformen beschrieben.

2. Dieses Kriterium gilt auch für alle Unterformen.

Kommentar: 1.1.1.3 Schmerzen der Zahnpulpa zurückzuführen auf eine Pulpitis können von schwach bis stark variieren und mit dem Schweregrad der Entzündung zusammenhängen. Eine schwere Entzündung der Zahnpulpa kann jedoch auch asymptomatisch verlaufen.

Die unter den im Folgenden aufgeführten Unterformen vorgeschlagenen diagnostischen Kriterien für eine reversible oder irreversible Pulpitis wurden nicht wissenschaftlich validiert, und das Vorliegen und die Charakteristika der Symptome korrelieren nicht ausreichend mit dem Zustand der Pulpa. Ist die Pulpa eine Zeitlang unmittelbar dem oralen Mikrobiom ausgesetzt gewesen, fehlt ihm die Fähigkeit zur Heilung und die Pulpitis gilt als irreversibel. Sofern eine Pulpitis deshalb in Verbindung mit Karies steht, gilt sie potenziell als reversibel, solange eine Zone funktionell intakten Dentins die Angriffsfläche der Bakterien vom vitalen Pulpagewebe trennt, und potenziell als irreversibel, wenn ein solcher Bereich nicht existiert.

1.1.1.3.1 Schmerzen der Zahnpulpa zurückzuführen auf eine reversible Pulpitis aufgrund einer Infektion des Dentins

\section{Diagnostische Kriterien:}

A. Zahnschmerz, der die Kriterien für 1.1.1.3 Schmerzen der Zahnpulpa zurückzuführen auf eine Pulpitis erfüllt

B. Es wurde eine reversible ${ }^{1}$ Pulpitis aufgrund einer Infektion des Dentins ${ }^{2}$ an dem betroffenen Zahn diagnostiziert

C. Nicht besser erklärt durch eine andere ICOP-Diagnose

\section{Anmerkungen:}

1. Die Diagnose einer reversiblen Schädigung basiert auf klinischen Befunden und/oder dem radiologischen Nachweis einer intakten Dentinzone über dem Dentin.
2. Auf eine Infektion deutet das Vorliegen von Karies oder Dentinsubstanz hin, die eine Zeitlang den Mikroorganismen der Mundhöhle ausgesetzt war.

Kommentar: Schmerzen zurückzuführen auf eine reversible Pulpitis werden in der Regel als leicht beschrieben, treten nicht spontan auf und sind durch Temperaturveränderungen provozierbar. Wird der Schmerz durch einen thermischen Reiz (Kälte oder Hitze) oder mechanischen Reiz (Sondieren, Bohren) ausgelöst, ist er üblicherweise von kurzer Dauer und hält nicht länger an als der Reiz selbst. Er reagiert auf peripher wirksame Analgetika (nichtsteroidale Antirheumatika [NSAR]).

1.1.1.3.1.1 Schmerzen der Zahnpulpa zurückzuführen auf eine reversible Pulpitis aufgrund einer Karies, die nicht die Pulpa erreicht Diagnostische Kriterien:

A. Zahnschmerz, der die Kriterien für 1.1.1.3.1 Schmerzen der Zahnpulpa zurückzuführen auf eine reversible Pulpitis aufgrund einer Infektion des Dentins erfüllt

B. Es wurde im betroffenen Zahn eine Karies diagnostiziert, ohne klinischen oder radiologischen Nachweis einer Verbindung zur Pulpa

C. Nicht besser erklärt durch eine andere ICOP-Diagnose

Kommentar: Zusätzlich zu einer thermischen Empfindlichkeit kann der Schmerz durch Druck auf das kariöse Dentin evoziert werden.

1.1.1.3.1.2 Schmerzen der Zahnpulpa zurückzuführen auf eine reversible Pulpitis aufgrund einer Fraktur im dentalen Hartgewebe mit freiliegendem Dentin

\section{Diagnostische Kriterien:}

A. Zahnschmerz, der die Kriterien für 1.1.1.3.1 Schmerzen der Zahnpulpa zurückzuführen auf eine reversible Pulpitis aufgrund einer Infektion des Dentins erfüllt

B. Klinischer und/oder radiologischer Nachweis von einem oder mehreren der folgenden Befunde im betroffenen Zahn, bei Freilegung von Dentin:

1. Fraktur mit alleiniger Beteiligung des Zahnschmelzes

2. Fraktur mit Beteiligung von Zahnschmelz und Dentin

3. Fraktur mit alleiniger Beteiligung des Wurzelzements

4. Fraktur mit Beteiligung von Wurzelzement und Dentin

5. Fraktur mit Beteiligung von Zahnschmelz, Wurzelzement und Dentin

C. Nicht besser erklärt durch eine andere ICOP-Diagnose

Kommentar: Zusätzlich zur thermischen Empfindlichkeit lässt sich der Schmerz durch Kratzen an der Oberfläche des infizierten Dentins evozieren. 
1.1.1.3.1.3 Schmerzen der Zahnpulpa zurückzuführen auf eine reversible Pulpitis aufgrund eines Risses oder Sprungs im Zahn ohne Hinweis auf fehlende Zahnsubstanz

\section{Diagnostische Kriterien:}

A. Zahnschmerz, der die Kriterien für 1.1.1.3.1 Schmerzen der Zahnpulpa zurückzuführen auf eine reversible Pulpitis aufgrund einer Infektion des Dentins sowie Kriterium C erfüllt

B. Es wurde ein Riss oder eine unvollständige Fraktur in dem betroffenen Zahn diagnostiziert, die den Zahnschmelz oder den Zahnschmelz und das Dentin betrifft ${ }^{1}$

C. Ein kausaler Zusammenhang lässt sich durch mindestens einen der folgenden Punkte nachweisen:

1. Stechender Schmerz beim Zubeißen ${ }^{2}$

2. Schmerz beim Lösen des okklusalen Beißdrucks oder bei äußerer Kraftanwendung ${ }^{2}$

3. Kälteüberempfindlichkeit ${ }^{2}$

D. Nicht besser erklärt durch eine andere ICOP-Diagnose

\section{Anmerkungen:}

1. Die Diagnose kann durch visuelle Diagnose eines Risses oder mehrerer Risse erfolgen, unterstützt durch Vergrößerungshilfen, starke Ausleuchtung oder Sichtbarmachung mit Farbstoff und/oder radiologisch oder durch andere bildgebende Verfahren.

2. Der Schmerz überdauert in jedem Fall nicht die Reizanwendung.

Kommentar: Bei Zähnen mit Rissen finden sich mitunter große Sondierungstiefen in Verbindung mit dem Rissverlauf.

1.1.1.3.2 Schmerzen der Zahnpulpa zurückzuführen auf eine irreversible Pulpitis aufgrund einer Infektion des Dentins

\section{Diagnostische Kriterien:}

A. Zahnschmerz, der die Kriterien für 1.1.1.3 Schmerzen der Zahnpulpa zurückzuführen auf eine Pulpitis erfüllt

B. Es wurde eine irreversible 1 Pulpitis aufgrund einer Infektion des Dentins ${ }^{2}$ in dem betroffenen Zahn diagnostiziert

C. Nicht besser erklärt durch eine andere ICOP-Diagnose

\section{Anmerkungen:}

1. Die Diagnose der Irreversibilität erfolgt durch den klinischen und/oder radiologischen Nachweis, dass die Pulpa von keiner intakten Dentinzone bedeckt ist und/oder durch einzelne oder mehrere der folgenden Schmerzcharakteristika:

a) Tritt spontan auf

b) Es handelt sich um einen Dauerschmerz

c) Besteht länger als die Stimulation (thermisch: Kälte oder Hitze; oder mechanisch: Sondieren oder Bohren) der Pulpa für mehr als ein paar Sekunden

d) Starker Schweregrad

e) Spricht wenig auf nichtsteroidale Antirheumatika (NSAR) an.

1. Belegt durch Vorliegen von Karies oder der Tatsache, dass Dentin für eine Zeit den Mikroorganismen der Mundhöhle ausgesetzt war.
Kommentar: Schmerzen zurückzuführen auf eine irreversible Pulpitis können sich durch Temperaturveränderungen verstärken und können auch mit Biss- oder Klopfempfindlichkeit verbunden sein. Nach seiner Auslösung überdauert der Schmerz den Reiz.

Das Vorliegen von Schmerzen korreliert jedoch nur bedingt mit dem Status der Zahnpulpa. Die Wertigkeit von Symptomen für die Ermittlung des Zustands der Pulpa (reversibel oder irreversibel entzündet) wird diskutiert und ist umstritten, wobei die wissenschaftliche Beweislage dürftig ist. Ein heftiger Dauerschmerz, der auf Analgetika (NSAR) nicht anspricht, kann ein Hinweis auf eine irreversible Entzündung sein und die Notwendigkeit einer invasiven Behandlung anzeigen.

1.1.1.3.2.1 Schmerzen der Zahnpulpa zurückzuführen auf eine irreversible Pulpitis aufgrund einer Karies, die bis zur Zahnpulpa reicht Diagnostische Kriterien:

A. Zahnschmerz, der die Kriterien für 1.1.1.3.2 Schmerzen der Zahnpulpa zurückzuführen auf eine irreversible Pulpitis aufgrund einer Infektion des Dentins erfüllt

B. Es wurde eine tiefe Karies in dem betroffenen Zahn diagnostiziert, mit klinischem und/oder radiologischem Nachweis eines wahrscheinlichen oder definitiven Mitbefalls der Pulpa

C. Nicht besser erklärt durch eine andere ICOP-Diagnose

1.1.1.3.2.2 Schmerzen der Zahnpulpa zurückzuführen auf eine irreversible Pulpitis aufgrund einer Fraktur dentaler Hartgewebe ohne Freilegung der Zahnpulpa

\section{Diagnostische Kriterien:}

A. Zahnschmerz, der die Kriterien für 1.1.1.3.2 Schmerzen der Zahnpulpa zurückzuführen auf eine irreversible Pulpitis aufgrund einer Infektion des Dentins erfüllt

B. Klinischer und/oder radiologischer Nachweis eines oder mehrerer der folgenden Punkte in dem betroffenen Zahn, ohne Freiliegen vitalen Pulpagewebes:

1. Fraktur unter Einbeziehung von Schmelz und Dentin (unkomplizierte Kronenfraktur), Fraktur mit Einbeziehung des Wurzelzements und Dentins (unkomplizierte Wurzelfraktur)

2. Fraktur unter Einbeziehung des Zahnschmelzes, Wurzelzements und Dentins (unkomplizierte Kronen-Wurzel-Fraktur)

C. Nicht besser erklärt durch eine andere ICOP-Diagnose

Kommentar: Zusätzlich zu einer schmerzhaften Reaktion auf thermische Reize lässt sich der Schmerz durch Kratzen an der Oberfläche des infizierten Dentins evozieren.

1.1.1.3.2.3 Schmerzen der Zahnpulpa zurückzuführen auf eine irreversible Pulpitis aufgrund eines Risses oder Sprungs im Zahn ohne Nachweis fehlender Zahnsubstanz

\section{Diagnostische Kriterien:}

A. Zahnschmerz, der die Kriterien für 1.1.1.3.2 Schmerzen der Zahnpulpa zurückzuführen auf eine irreversible Pulpitis aufgrund einer Infektion des Dentins sowie Kriterium C erfüllt

B. Es wurde ein Riss oder eine unvollständige Fraktur des betroffenen Zahns unter Einbeziehung des Zahnschmelzes oder des Zahnschmelzes und Dentins diagnostiziert ${ }^{1}$ 
C. Ein kausaler Zusammenhang lässt sich durch mindestens einen der folgenden Punkte nachweisen:

1. Stechender Schmerz beim Zubeißen ${ }^{2}$

2. Schmerz beim Lösen des okklusalen Beißdrucks oder von Kräften, die von außen einwirken ${ }^{2}$

3. Kälteüberempfindlichkeit ${ }^{2}$

D. Nicht besser erklärt durch eine andere ICOP-Diagnose

\section{Anmerkungen:}

1. Die Diagnose kann durch visuelle Identifikation eines Risses oder mehrerer Risse erfolgen, unterstützt durch Vergrößerung, starke Ausleuchtung oder Sichtbarmachung mit Farbstoff und/oder radiologisch oder durch andere bildgebende Verfahren.

2. Der Schmerz überdauert häufig die Reizanwendung.

Kommentar: Risse in Zähnen können zu einem stechenden Schmerz beim Zubeißen, zu unerklärlicher Kälteempfindlichkeit oder Schmerz bei Druckentlastung führen oder ein tiefes Eindringen der Sonde an der Rissstelle erlauben. Der Schmerz hält in der Regel über die Reizeinwirkung hinaus an.

1.1.1.3.3 Schmerzen der Zahnpulpa zurückzuführen auf eine irreversible Pulpitis aufgrund einer Infektion der Zahnpulpa

\section{Diagnostische Kriterien:}

A. Zahnschmerz, der die Kriterien für 1.1.1.3 Schmerzen der Zahnpulpa zurückzuführen auf eine Pulpitis sowie Kriterium C erfüllt

B. Es liegt eine Infektion' der Zahnpulpa des betroffenen Zahnes vor

C. Der Schmerz hat sich in einem engen zeitlichen Zusammenhang mit der Infektion entwickelt oder führte zu ihrer Entdeckung

D. Nicht besser erklärt durch eine andere ICOP-Diagnose

\section{Anmerkung:}

1. Die Infektion liegt nahe, da die Pulpa eine Zeitlang dem Mikrobiom der Mundhöhle ausgesetzt war.

1.1.1.3.3.1 Schmerzen der Zahnpulpa zurückzuführen auf eine irreversible Pulpitis aufgrund einer kariösen Freilegung und Infektion der Zahnpulpa

\section{Diagnostische Kriterien:}

A. Zahnschmerz, der die Kriterien für 1.1.1.3.3 Schmerzen der Zahnpulpa zurückzuführen auf eine irreversible Pulpitis aufgrund einer Infektion der Zahnpulpa erfüllt

B. Es wurde eine tiefe Karies in dem betroffenen Zahn diagnostiziert und der klinische und/oder radiologische Nachweis für eine Ausdehnung bis zur Pulpa erbracht

C. Nicht besser erklärt durch eine andere ICOP-Diagnose

Kommentar: Histologische Untersuchungen zeigen, dass eine Entzündung wahrscheinlich irreversibel ist, wenn die kariöse Läsion (bakterielle Angriffsfront) die Zahnpulpa erreicht. Die Beurteilung stützt sich auf das klinische und radiologische Erscheinungsbild. Wenn keine intakte funktionsfähige Zone mit Dentinsubstanz zwischen dem kariösen Dentin und der Zahnpulpa zu sehen ist, lässt sich daraus schließen, dass die Mikroben in direktem Kontakt mit dem Pulpagewebe stehen und dieses infiziert haben, was zu einer schweren Entzündung geführt hat. Es sollte beachtet werden, dass diese Erkrankung in vielen Fällen symptomfrei verlaufen kann.

1.1.1.3.3.2 Schmerzen der Zahnpulpa zurückzuführen auf eine irreversible Pulpitis aufgrund einer Fraktur der dentalen Hartgewebe mit freiliegender Zahnpulpa

Diagnostische Kriterien:

A. Zahnschmerz, der die Kriterien für 1.1.1.3.3 Schmerzen der Zahnpulpa zurückzuführen auf eine irreversible Pulpitis aufgrund einer Infektion der Zahnpulpa sowie Kriterium C erfüllt

B. Klinischer und/oder radiologischer Nachweis von einem der folgenden Frakturtypen in dem betroffenen Zahn, mit Freilegung von vitalem Pulpagewebe:

1. Fraktur, die Zahnschmelz, Dentin und Pulpa betrifft (komplizierte Kronenfraktur)

2. Fraktur, die Wurzelzement, Dentin und Pulpa betrifft (komplizierte Wurzelfraktur)

3. Fraktur, die Zahnschmelz, Wurzelzement, Dentin und Pulpa betrifft (komplizierte Kronen-Wurzel-Fraktur)

C. Der Schmerz hat sich in einem engen zeitlichen Zusammenhang mit der Fraktur entwickelt oder zu ihrer Entdeckung geführt

D. Nicht besser erklärt durch eine andere ICOP-Diagnose

Kommentar: Neben einer Auslösung durch eine thermische Empfindlichkeit kann der Schmerz durch mechanische Stimulation der freiliegenden Zahnpulpa oder des benachbarten Dentins evoziert werden.

1.1.1.3.4 Schmerzen der Zahnpulpa zurückzuführen auf eine Pulpitis aufgrund einer externen zervikalen Wurzelresorption

Diagnostische Kriterien:

A. Zahnschmerz, der die Kriterien für 1.1.1.3 Schmerzen der Zahnpulpa zurückzuführen auf eine Pulpitis sowie Kriterium C erfüllt

B. Es wurde im betroffenen Zahn durch klinische und/oder radiologische Beobachtung eine externe zervikale Wurzelresorption diagnostiziert

C. Der Schmerz entstand in zeitlichem Zusammenhang mit dem Beginn der Resorption oder führte zu deren Entdeckung

D. Nicht besser erklärt durch eine andere ICOP-Diagnose

Kommentar: Die zervikale Wurzelresorption ist ein Prozess, bei dem Wurzeldentin durch odontoklastische Aktivität resorbiert wird. Die Erkrankung verläuft solange asymptomatisch, bis die Zahnpulpa befallen ist, was gewöhnlich erst spät im Verlauf eintritt.

Eine Sekundärinfektion im resorbierten Areal stimuliert eine Entzündungsreaktion in der benachbarten Pulpa, die reversibel oder irreversibel sein kann. Aus technischen und/oder prognostischen Gründen umfasst das Management der Resorption gewöhnlich eine Behandlung der Zahnpulpa (Pulpektomie) unabhängig vom Grad der Entzündung der Dentinsubstanz.

Zusätzlich zu einer Auslösung durch thermische Reize kann der Schmerz durch Druck auf den resorptiven Defekt im Dentin evoziert werden. 
1.1.1.3.5 Schmerzen der Zahnpulpa zurückzuführen auf eine Pulpitis aufgrund einer anderen Ursache

Diagnostische Kriterien: Zahnschmerz in einem oder mehreren Zähnen, der die Kriterien für 1.1.1.3 Schmerzen der Zahnpulpa zurückzuführen auf eine Pulpitis sowie Kriterium C erfüllt

A. Es wurde eine andere Erkrankung als die unter 1.1.1.3.1 bis 1.1.1.3.4 genannten diagnostiziert, die bekanntermaßen Schmerzen der Zahnpulpa ${ }^{1}$ auslösen kann

B. Ein ursächlicher Zusammenhang ist aufgrund des anatomischen, funktionellen und/oder zeitlichen Zusammenhangs plausibel

C. Nicht besser erklärt durch eine andere ICOP-Diagnose

\section{Anmerkung:}

1. Zum Beispiel lassen einige Berichte in der Fachliteratur darauf schließen, dass Pulpitis und Schmerzen der Zahnpulpa sekundär als Folge von neurovaskulären Ereignissen auftreten können (neurogene Entzündung).

Kommentar: Die Schmerzsymptome können von einer Überempfindlichkeit des Dentins bis zu einem anhaltenden Schmerz reichen, der auf eine Pulpitis verweist, und treten oft in Begleitung autonomer Zeichen auf (siehe 5.1 Orofaziale Migräne).

1.1.1.4 Schmerzen der Zahnpulpa zurückzuführen auf eine systemische Ursache

\section{Diagnostische Kriterien:}

A. Zahnschmerz an einem oder mehreren Zähnen, der die Kriterien für 1.1.1 Schmerzen der Zahnpulpa sowie Kriterium C erfüllt

B. Es wurde eine systemische Störung oder Erkrankung diagnostiziert, die bekannterweise Schmerzen der Zahnpulpa ${ }^{1}$ auslösen kann

C. Der ursächliche Zusammenhang mit dem Schmerz ist klinisch plausibel

D. Nicht besser erklärt durch eine andere ICOP-Diagnose

\section{Anmerkung:}

1. Ein Beispiel ist die Sichelzellanämie.

Kommentar: Schmerzen der Zahnpulpa können die Folge einer systemischen Erkrankung sein, die eine Veränderung im Zustand der Zahnpulpa bewirkt. So können etwa hämolytische Krisen bei Sichelzellanämie Zahnschmerzen hervorrufen. Es wurde von Pulpanekrosen bei Patienten mit Sichelzellanämie berichtet, die sekundär als Folge von Gefäßverschlüssen auftraten. Das Phänomen des „Sichelzellzahnschmerzes" kann dann auftreten, wenn Sichelzellen in der Gefäßversorgung der Zahnpulpa steckenbleiben und den Blutfluss im Bindegewebe der Pulpa behindern. Dies führt zu Sauerstoffmangel, Symptomen einer Pulpitis, Zelltod und letztlich einem Vitalitätsverlust des Zahnes.

Wenn eine systemische Erkrankung zu Schmerzen der Zahnpulpa führt, kommt es nicht selten vor, dass mehrere Zähne betroffen sind.

\subsubsection{Parodontale Schmerzen}

Beschreibung: Schmerz verursacht durch eine Läsion oder Erkrankung mit Beteiligung des Zahnbetts: die Wurzelhaut und/ oder das benachbarte alveoläre (periradikuläre) Knochengewebe.

\section{Diagnostische Kriterien:}

A. Jeglicher Schmerz im Zahnbett ${ }^{1}$, der Kriterium C erfüllt

B. Eine Läsion, eine Erkrankung oder ein Trauma² die/das bekanntermaßen parodontale Schmerzen auslösen kann, wurde klinisch, laborchemisch und/oder mittels Bildgebung nachgewiesen

C. Ein ursächlicher Zusammenhang lässt sich durch beide der folgenden Kriterien nachweisen:

1. Die Lokalisation des Schmerzes entspricht dem Situs der Läsion, der Erkrankung oder des Traumas ${ }^{1}$

2. Der Schmerz verschlimmert sich bei Einwirkung eines physischen Reizes ${ }^{3}$ auf den betroffenen Zahn (horizontal oder vertikal) oder auf das über der Zahnwurzel liegende Gewebe

D. Nicht besser erklärt durch eine andere ICOP-Diagnose

\section{Anmerkungen:}

1. Der Schmerz kann auch auf andere ipsilaterale orofaziale Regionen übertragen werden und/oder dorthin ausstrahlen.

2. Die Läsion, die Erkrankung oder das Trauma wird bei jeder Unterform spezifiziert.

3. Der Reiz kann mechanischer, thermischer oder chemischer Art sein, wie bei den jeweiligen Unterformen spezifiziert.

Kommentar: Parodontale Schmerzen können von allen Arten parodontaler Verletzungen oder Erkrankungen begleitet sein. Der Schmerz ist vorrangig entzündlicher Natur und Sekundärfolge äußerer oder innerer Ereignisse.

\subsubsection{ParodontaleSchmerzen zurückzuführen aufeine Parodontitis (parodontale Entzündung)}

\section{Diagnostische Kriterien:}

A. Schmerzen, die die Kriterien für 1.1.2 Parodontale Schmerzen sowie Kriterium C erfüllen

B. Es wurde eine parodontale Entzündung ${ }^{1}$ diagnostiziert

C. Ein ursächlicher Zusammenhang ist aufgrund der anatomischen, funktionellen und/oder zeitlichen Verbindung plausibel $^{2}$

D. Nicht besser erklärt durch eine andere ICOP-Diagnose

\section{Anmerkungen:}

1. Die Entzündung kann auf ein Trauma oder eine Infektion zurückgehen und wird bei jeder Unterform spezifiziert.

2. Dieses Kriterium gilt auch für alle Unterformen.

Kommentar: 1.1.2.1 Parodontale Schmerzen zurückzuführen auf eine Parodontitis wird nach Entzündungsgrund in Unterkategorien untergliedert.

Eine (marginale wie auch apikale) Parodontitis verläuft meist asymptomatisch, kann aber auch mit Schmerzen einhergehen und mitunter mit einer beobachtbaren Schwellung. In solchen Fällen wird der Schmerz durch einen mechanischen Reiz wie Beißen oder 
Kauen ausgelöst und lässt sich für den Patienten in der Regel leicht lokalisieren. Es kann auch ein Spontanschmerz auftreten, der sich üblicherweise über Stunden erstreckt. Die Schmerzintensität kann von leicht bis stark reichen. Der Schmerz lässt sich durch Klopfen oder durch Anwendung von Druck auf den Zahn reproduzieren.

Dieser Schmerztyp kann auch von gingivalen Schmerzen begleitet sein.

1.1.2.1.1 Parodontale Schmerzen zurückzuführen auf eine traumatisch bedingte parodontale Entzündung

\section{Diagnostische Kriterien:}

A. Schmerzen, die die Kriterien für 1.1.2.1 Parodontale Schmerzen zurückzuführen auf eine Parodontitis sowie Kriterium C erfüllen

B. Bei einem Trauma oder einer Verletzung ${ }^{1}$ war das betroffene parodontale Gewebe involviert

C. Der Schmerz hat sich innerhalb von Minuten bis Tagen nach einem Trauma oder einer Verletzung entwickelt

D. Nicht besser erklärt durch eine andere ICOP-Diagnose

\section{Anmerkung:}

1. Die Verletzung kann unfallbedingt oder nicht unfallbedingt sein, von anderen oder selbst zugefügt oder iatrogen.

Kommentar: Eine traumatische Verletzung von parodontalem Gewebe verursacht eine akute Entzündung des Zahnhalteapparats und kann in unterschiedlichem Umfang schmerzhaft sein (von leicht bis stark); der Schmerz verschlimmert sich durch mechanische Provokation des Zahns. Es kann Spontanschmerz auftreten.

Von unfallbedingten Zahntraumen oder -verletzungen sind 10$30 \%$ der Bevölkerung betroffen, wobei diese fast ausschließlich an Schneidezähnen auftreten (davon Oberkiefer 75-80\% und Unterkiefer 20-25\%). Als Inzidenz wurden zwei bis drei Frontzahnverletzungen/100 Schulkinder/Jahr berichtet, und die Prävalenz traumatisierter bleibender Zähne bei Kindern und Jugendlichen liegt Berichten zufolge bei 6-34 \%. Epidemiologische Daten deuten darauf hin, dass leichte Zahntraumen zwar am häufigsten vorkommen, dass bei einer Population im Alter von 6-50 Jahren jedoch etwa $3 \%$ bleibender Schneidezähne von einer traumatischen Verletzung betroffen waren, die schwer genug ausfiel, um schmerzhaft zu sein.

Zu den iatrogenen Ursachen gehören durch die Behandlung unbeabsichtigte Zahnverletzungen, aber auch Mikrotraumen, etwa durch Veränderungen im Hinblick auf die Okklusion oder Artikulation nach einer Zahnbehandlung, sowie Schädigungen am Zahnhalteapparat, etwa durch parodontalchirurgische Eingriffe.

1.1.2.1.1 Parodontale Schmerzen zurückzuführen auf eine traumatisch bedingte parodontale Entzündung werden deshalb gemäß der Art des Traumas oder der Verletzung in Unterkategorien untergliedert.

1.1.2.1.1.1 Parodontale Schmerzen zurückzuführen auf okklusale Frühkontakte oder Interferenzen

\section{Diagnostische Kriterien:}

A. Schmerzen, die die Kriterien für 1.1.2.1.1 Parodontale Schmerzen zurückzuführen auf eine traumatisch bedingte parodontale

Entzündung sowie die Kriterien C und D erfüllen
B. Es sind veränderte okklusale Bedingungen aufgetreten, verbunden mit einem hieraus resultierenden Frühkontakt oder okklusaler Interferenz, erkennbar an mindestens einem der folgenden Punkte:

1. Primärkontakt, der die Okklusion oder Artikulation eines Zahns betrifft

2. Hypermobilität eines Zahnes

C. Der Schmerz hat sich Stunden bis Tage nach Auftreten der veränderten okklusalen Bedingungen entwickelt

D. Der Schmerz lässt sich durch mechanische Provokation ${ }^{1}$ reproduzieren

E. Nicht besser erklärt durch eine andere ICOP-Diagnose

\section{Anmerkung:}

1. Die mechanische Provokation kann in Druck oder Klopfen auf den betroffenen Zahn bestehen.

Kommentar: Parodontale Schmerzen zurückzuführen auf okklusale Faktoren sind mit einer Sensibilisierung parodontaler Nozizeptoren und einer Entzündungsreaktion aufgrund exzessiver Zahnbelastung verbunden.

Anamnestisch findet sich eine kurz zuvor stattgefundene Zahnrestauration, Zahnextraktion oder sonstige Veränderung in der Okklusion oder Artikulation. Der Patient berichtet eventuell, dass der Zahn sich so anfühlt, als stünde er leicht über. Klinisch wird ein Primärkontakt bei der Okklusion oder Artikulation festgestellt. Der Schmerz lässt sich durch Klopfen oder Anwendung von Druck auf den Zahn reproduzieren. Der Zahn kann eine erhöhte Mobilität aufweisen. In diesem Fall kann die radiologische Untersuchung einen erweiterten Parodontalspalt zeigen.

\subsection{Postoperative parodontale Schmerzen}

\section{Diagnostische Kriterien:}

A. Schmerzen, die die Kriterien für 1.1.2.1.1 Parodontale Schmerzen zurückzuführen auf eine traumatisch bedingte parodontale Entzündung sowie Kriterium C erfüllen

B. Es hat ein chirurgischer Eingriff stattgefunden, bei dem der Zahnhalteapparat involviert war

C. Der Schmerz hat sich innerhalb von Stunden bis Tagen nach dem chirurgischen Eingriff entwickelt

D. Nicht besser erklärt durch eine andere ICOP-Diagnose

Kommentar: Postoperative parodontale Schmerzen sind iatrogen, verursacht durch eine chirurgisch herbeigeführte Schädigung mit nachfolgender Entzündung. Der Schmerz ist üblicherweise leicht bis mittelstark und kann in Begleitung einer klinisch beobachtbaren Schwellung und gelegentlich Eiterbildung auftreten.

Wenn eine normale physiologische (primäre) Heilung auftritt, ist die Schmerzdauer üblicherweise kurz (1-2 Wochen). Gelegentlich werden länger anhaltende Schmerzen aufgrund von Sekundärheilung und/oder einer postoperativen Infektion beobachtet, dauert in der Regel jedoch nicht länger als 3 Monate. 
1.1.2.1.1.3 Parodontale Schmerzen zurückzuführen auf ein unfallbedingtes Zahntrauma

\section{Diagnostische Kriterien:}

A. Schmerzen, die die Kriterien für 1.1.2.1.1 Parodontale Schmerzen zurückzuführen auf eine traumatisch bedingte parodontale Entzündung sowie Kriterium C erfüllen

B. Ein Zahn ist von einem Unfalltrauma' betroffen, mit klinischem und/oder radiologischem Nachweis eines oder mehrerer der folgenden Punkte:

1. Konkussion

2. Subluxation

3. Laterale Luxation

4. Intrusion

5. Extrusion

6. Avulsion

7. Wurzelfraktur ${ }^{2}$

C. Der Schmerz hat sich innerhalb von Minuten bis Tagen nach dem Trauma entwickelt

D. Nicht besser erklärt durch eine andere ICOP-Diagnose

\section{Anmerkungen:}

1. Mögliche Unfallursache im Falle einer Wurzelfraktur ist eine exzessive Belastung des Zahns.

2. Die Wurzelfraktur kann horizontal oder vertikal verlaufen.

Kommentar: Von unfallbedingten Zahntraumen oder -verletzungen sind 10-30\% der Bevölkerung betroffen. Diese treten fast ausschließlich an Schneidezähnen auf (Oberkiefer 75-80\% und Unterkiefer 20-25\%). Als Inzidenz wurden zwei bis drei Frontzahnverletzungen/100 Schulkinder/Jahr berichtet, und die Prävalenz traumatisierter bleibender Zähne bei Kindern und Jugendlichen liegt Berichten zufolge bei 6-34 \%. Epidemiologische Daten deuten darauf hin, dass leichte Traumen zwar am häufigsten vorkommen, dass jedoch etwa $3 \%$ bleibender Schneidezähne bei einer Population im Alter von 6-50 Jahren von einer traumatischen Verletzung betroffen waren, die schwer genug war, um schmerzhaft zu sein.

Konkussions-, Subluxations- und Extrusionstraumen können auch mit einer Verletzung der Zahnpulpa einhergehen, und parodontale Schmerzen können zusammen mit Schmerzen der Zahnpulpa auftreten (siehe 1.1.1 Schmerzen der Zahnpulpa).

Laterale Luxations- und Intrusionstraumen bewirken auch Verletzungen der Zahnpulpa sowie des Alveolarfortsatzes, und parodontale Schmerzen können zusammen mit Schmerzen der Zahnpulpa und Schmerzen im Kieferknochen auftreten (siehe 1.1.1 Schmerzen der Zahnpulpa und 1.2.3 Schmerzen im Kieferknochen).

Ein Avulsionstrauma kann auch mit einer Verletzung des Alveolarfortsatzes einhergehen, und parodontale Schmerzen können zusammen mit 1.2.3 Schmerzen im Kieferknochen auftreten.

Eine Wurzelfraktur ist eine Verletzung des Zahnhartgewebes, die bis zum Pulparaum reichen kann. Wenn die Zahnpulpa beteiligt ist, ist sie unmittelbar der bakteriellen Besiedlung aus der Mundhöhle ausgesetzt und entzündet sich schnell. Wenn die Pulpa vital ist, kann der Schmerz mit 1.1.1.3.3.2 Schmerzen der Zahnpulpa zurückzuführen auf eine irreversible Pulpitis aufgrund einer Fraktur der dentalen Hartgewebe mit freiliegender Zahnpulpa zusammenfallen. Neben einem unfallbedingten Trauma sind andere häufige Grün- de für eine Wurzelfraktur unter anderem die exzessive Belastung eines Zahns nach Wurzelkanalbehandlung, üblicherweise mit einem Stiftaufbau.

Ein Frontzahntrauma verursacht häufig parodontale Schmerzen. Das klinische und radiologische Erscheinungsbild und die Charakteristika und der Schweregrad des Schmerzes hängen von der Natur und Schwere der traumatischen Verletzung ab. Folgender Link gibt eine kurze Beschreibung der in der Zahnarztpraxis üblichen Traumadiagnosen (aus dem Dental Trauma Guide; https:// dentaltraumaguide.org [abgerufen Januar 2020]).

Parodontale Schmerzen aufgrund einer Konkussion werden durch eine unfallbedingte Verletzung des Zahnbetts und eine nachfolgende Entzündung verursacht. Der Zahn zeigt eine normale Mobilität und wird nicht aus dem dazugehörigen Zahnfach gelöst. Sofern zuvor keine Wurzelkanalbehandlung stattgefunden hat, lässt sich am Zahn in der Regel eine vitale Pulpa nachweisen. Die Bildgebung zeigt unauffällige periradikuläre Bedingungen.

Parodontale Schmerzen aufgrund einer Subluxation werden durch eine unfallbedingte Verletzung des Zahnbetts mit nachfolgender Entzündung verursacht. Der Zahn zeigt eine erhöhte Mobilität, wird aber nicht aus der Verankerung im dazugehörigen Zahnfach gelöst. Die klinischen Befunde zeigen unter anderem eine Blutung aus dem Sulcus gingivalis. Der Zahn reagiert in etwa $50 \%$ der Fälle auf den Pulpasensibilitätstest. Die radiologische Untersuchung kann eine Verbreiterung des Parodontalspalts zeigen.

Parodontale Schmerzen aufgrund einer lateralen Luxation werden durch eine unfallbedingte Verletzung des Zahnbetts und eine nachfolgende Entzündung ausgelöst. Der Zahn wird seitwärts aus der Verankerung in seiner Alveole geschoben, in Kombination mit einem Splitterbruch oder Bruch des bukkalen oder lingualen/palatinalen Alveolarfortsatzes. Die Wurzelhaut (Desmodont) ist teilweise oder komplett durchtrennt, und man beobachtet eine Blutung aus dem Sulkus. Der Zahn zeigt gewöhnlich eine verminderte Mobilität und kann die Okklusion und/oder Artikulation behindern. Der Zahn reagiert in der Regel nicht auf einen Pulpavitalitätstest. Die radiologische Untersuchung zeigt eine Abweichung im Hinblick auf die Weite des Parodontalspalts in Abhängigkeit von der Projektion.

Parodontale Schmerzen aufgrund einer Intrusion entstehen durch eine unfallbedingte Verletzung des Zahnbetts und einer nachfolgenden Entzündung. Der Zahn wird axial in den Alveolarfortsatz verschoben und wirkt so kürzer als der benachbarte Zahn. Die Verletzung tritt in Begleitung eines Splitterbruchs oder Bruchs der Alveole auf. Zu den weiteren klinischen Befunden können verminderte Mobilität und ein hohes Geräusch beim Klopftest gehören. Der Zahn reagiert gewöhnlich nicht auf den Pulpavitalitätstest. Die radiologische Untersuchung zeigt das Fehlen (oder die verminderte Breite) das Parodontalspalts im gesamten Zahn oder in einem Teil des Zahns.

Parodontale Schmerzen aufgrund einer Extrusion entstehen durch eine unfallbedingte Verletzung des Zahnhalteapparats und einer nachfolgenden Entzündung. Der Zahn wird axial in den Alveolarfortsatz und teilweise aus seiner Alveole heraus verschoben und wirkt deshalb verlängert. Der Parodontalspalt ist teilweise oder ganz durchtrennt, und es kommt zu einer Blutung aus dem Sulkus, aber die Alveole ist intakt. Der Zahn weist eine erhöhte Mobilität auf und kann die Okklusion/Artikulation behindern. Der Zahn re- 
agiert gewöhnlich nicht auf den Pulpavitalitätstest. Die radiologische Untersuchung ergibt eine erhöhte Weite des Parodontalspalts.

Parodontale Schmerzen aufgrund einer Avulsion entstehen durch eine unfallbedingte Verletzung des Zahnhalteapparats und eine nachfolgende Entzündung. Der Zahn wird völlig aus der Alveole gehoben, die dem Befund nach leer oder mit einem Blutgerinnsel gefüllt ist. Der umgebende Alveolarfortsatz kann frakturiert sein.

Parodontale Schmerzen entstehen aufgrund von Wurzelbruch durch Luxation oder Fragmente und/oder eine nachfolgende Infektion, die eine parodontale Entzündung verursacht. Anamnestisch kann sich ein unfallbedingtes Traumaereignis finden. Das koronale Fragment kann verschoben sein, und der Zahn kann länger wirken als die benachbarten Zähne. Er kann eine erhöhte Mobilität zeigen und die Okklusion/Artikulation behindern. Es kann eine tiefe lokale Zahnfleischtasche vorliegen. Die Bildgebung zeigt eine vertikale oder horizontale Fraktur, die sich auf die Wurzel beschränkt. Sofern zuvor keine Wurzelbehandlung erfolgt ist, kann der Zahn auf einen Pulpasensibilitätstest reagieren.

1.1.2.1.1.4 Parodontale Schmerzen zurückzuführen auf ein sonstiges Trauma oder eine sonstige Verletzung

\section{Diagnostische Kriterien:}

A. Schmerzen, die die Kriterien für 1.1.2.1.1 Parodontale Schmerzen zurückzuführen auf eine traumatisch bedingte parodontale Entzündung und Kriterium C erfüllen

B. Der betroffene Zahn oder die betroffenen Zähne war(en) in ein nicht unfall- oder gewaltbedingtes Trauma ${ }^{1}$ involviert

C. Der Schmerz hat sich in einem engen zeitlichen Zusammenhang mit dem Trauma entwickelt oder führte zu seiner Entdeckung

D. Nicht besser erklärt durch eine andere ICOP-Diagnose

\section{Anmerkung:}

1. Anamnestische, klinische, radiologische oder sonstige Befunde bildgebender Verfahren belegen ein Trauma, das bekanntermaßen eine parodontale Entzündung auslösen kann, etwa unzureichende Kühlung während restaurativer zahnärztlicher Maßnahmen, interdentale Fremdkörperimpaktion (einschließlich Nahrungsmittelimpaktion), mangelhafte Zahnrestauration oder apikal extrudiertes endodontisches Material. Zu den klinischen Befunden können Zeichen einer akuten Entzündung gehören (Schwellung, Rötung, Eiterbildung), eine erhöhte Zahnmobilität und/oder eine lokale tiefe Zahnfleischtasche. Sofern nicht wurzelbehandelt, lässt sich am Zahn üblicherweise eine vitale Pulpa nachweisen. Bildgebende Verfahren können einen marginalen lokalen Knochenschwund zeigen, von dem die periapikale Region mitbetroffen sein kann.

1.1.2.1.2 Parodontale Schmerzen zurückzuführen auf eine apikale Parodontitis aufgrund einer endodontischen Erkrankung

\section{Diagnostische Kriterien:}

A. Schmerzen, die die Kriterien für 1.1.2.1 Parodontale Schmerzen zurückzuführen auf eine Parodontitis erfüllen

B. Es wurde eine apikale Parodontitis aufgrund einer endodontischen Erkrankung diagnostiziert

C. Nicht besser erklärt durch eine andere ICOP-Diagnose
Kommentar: Eine endodontische Erkrankung (d.h. pulpale und periapikale Erkrankung) tritt häufig in Begleitung von Schmerzen auf, die leicht bis stark sein können. Parodontale Schmerzen aufgrund einer endodontischen Erkrankung gehen mit einer pulpalen, periapikalen, juxtaradikulären und/oder periradikulären Entzündung einher. Eine durchbrochene Barriere zur Mundhöhle, oft verursacht durch Karies, und nachfolgender Bakterienbefall von Pulpa und Wurzelkanalsystem sind die Hauptgründe für eine Entzündung der Zahnpulpa und des periapikalen Gewebes.

Diese Art von Schmerz kann auch das Zahnfleisch in Mitleidenschaft ziehen.

Eine endodontische Erkrankung, einschließlich einer periapikalen, juxtaradikulären oder periradikulären Entzündung, kann auch ohne klinische Symptome vorliegen.

1.1.2.1.2.1 Parodontale Schmerzen zurückzuführen auf eine Entzündung der Zahnpulpa

\section{Diagnostische Kriterien:}

A. Schmerzen, die die Kriterien für 1.1.2.1.2 Parodontale Schmerzen zurückzuführen auf eine apikale Parodontitis aufgrund einer endodontischen Erkrankung erfüllen

B. Es wurde aufgrund des Nachweises von beiden der folgenden Punkte eine Pulpitis (reversibler oder irreversibler Art) diagnostiziert:

1. Nachweis einer Zahnerkrankung des betroffenen Zahnes, die erfahrungsgemäß eine Pulpitis auslösen kann

2. Nachweis der Pulpavitalität durch Reaktion auf Pulpavitalitätstest

C. Nicht besser erklärt durch eine andere ICOP-Diagnose

Kommentar: Parodontale Schmerzen als Sekundärfolge einer Entzündung des Dentins treten in Verbindung mit einer symptomatischen Pulpitis auf. Die parodontale Entzündung konzentriert sich auf die periapikale Region.

Die Pulpa ist vital und der Zahn reagiert deshalb üblicherweise auf den Pulpavitalitätstest. Der Zahn ist oft klopfempfindlich. Es können klinische Befunde wie tiefe Karies, tiefe/mangelhafte Restauration oder eine externe zervikale Wurzelresorption vorliegen. Bildgebende Verfahren können einen Nachweis für eine diffuse periapikale Knochenresorption oder -sklerose erbringen.

Der Fachliteratur zufolge besteht eine schwache Verbindung zwischen dem tatsächlichen Zustand der Zahnpulpa und des Zahnhalteapparats (Histologie) und diagnostischen Befunden, einschließlich aktueller und vorangegangener Symptome wie Besonderheiten des geschilderten Zahnschmerzes, klinischer Beobachtungen und Untersuchungsergebnisse. Die aktuelle Diagnostik stützt sich größtenteils auf Expertenmeinung und einige wenige Studien mit Qualitätsdefiziten.

1.1.2.1.2.1 Parodontale Schmerzen zurückzuführen auf eine Entzündung der Zahnpulpa erfüllen häufig ebenfalls die Kriterien für 1.1.1.3 Schmerzen der Zahnpulpa zurückzuführen auf eine Pulpitis. In diesem Fall sollten beide Diagnosen gestellt werden. 
1.1.2.1.2.2 Parodontale Schmerzen zurückzuführen auf eine endodontische Infektion

\section{Diagnostische Kriterien:}

A. Schmerzen, die die Kriterien für 1.1.2.1.2 Parodontale Schmerzen zurückzuführen auf eine apikale Parodontitis aufgrund einer endodontischen Erkrankung erfüllen

B. Es wurde durch beide der folgenden Punkte eine partielle oder komplette Pulpanekrose und eine endodontische Infektion im Zahn diagnostiziert:

1. Nicht vitale Pulpa, nachgewiesen durch:

a) direkte Inspektion oder ausbleibendes Reagieren auf einen Pulpavitalitätstest oder

b) einen zuvor ausgeräumten Wurzelkanal

2. Klinischer ${ }^{1}$ und/oder radiologischer ${ }^{2}$ Nachweis einer apikalen Entzündung ${ }^{3}$

C. Nicht besser erklärt durch eine andere ICOP-Diagnose

\section{Anmerkungen:}

1. Zum klinischen Nachweis gehören Klopf- und/oder Druckempfindlichkeit und/oder Empfindlichkeit bei apikaler Palpation.

2. Der radiologische Nachweis beinhaltet eine apikale oder juxtaradikuläre Radioluzenz oder Sklerose.

3. Die apikale Entzündung geht mit einer symptomatischen apikalen Parodontitis oder einem akuten apikalen Abszess einher.

Kommentar: Parodontale Schmerzen aufgrund einer endodontischen Infektion treten im Zusammenhang mit einer nichtvitalen Pulpa (oder einem zuvor mit einer Wurzelfüllung versorgten Zahn) und einer Infektion der Pulpahöhle auf. Die Pulpa ist ganz oder teilweise nekrotisch (es sei denn, bei dem Zahn wurde vorher eineWurzelkanalbehandlung durchgeführt), und der Zahn reagiert üblicherweise nicht auf den Pulpasensibilitätstesttest. Obwohl er lokalisiert ist, überträgt sich der Schmerz häufig auf andere orofazialen Areale derselben Seite und/oder strahlt dorthin aus, insbesondere bei starken Schmerzen. Der Schmerz lässt sich durch Klopfen oder Druckausübung auf den Zahn und/oder die benachbarte periapikale vestibuläre Region reproduzieren. Die Bildgebung erbringt in der Regel den Nachweis einer lokalen periapikalen Knochenresorption.

Die Entzündungsreaktion im periapikalen Gewebe wird durch eine Wurzelkanalinfektion mit einer Mischflora ausgelöst. Eine erhöhte Inzidenz von Schmerz und Schwellung bei einer apikalen Parodontitis geht mit dem Auftreten spezifischer Anaerobier der Gattung Porphyromonas, Peptostreptococcus und Prevotella einher. Bei lokaler Infektionsausbreitung kann ein periapikaler Abszess entstehen.

1.1.2.1.2.2.1 Parodontale Schmerzen zurückzuführen auf eine intraradikuläre endodontische Infektion

\section{Diagnostische Kriterien:}
A. Schmerzen, die die Kriterien für 1.1.2.1.2.2 Parodontale Schmerzen zurückzuführen auf eine endodontische Infektion er- füllen

B. An einem Zahn findet sich eine Wurzelkanalinfektion ${ }^{1}$

C. Nicht besser erklärt durch eine andere ICOP-Diagnose

\section{Anmerkung:}

1. Die Infektion kann von Bakterien, Viren, Pilzen oder sonstigem ausgelöst sein.

Kommentar: In den meisten Zähnen mit infizierter nekrotischer Pulpa ist die Infektion auf das Wurzelkanalsystem beschränkt.

Eine erfolgreiche Behandlung der Infektion führt in der Regel zum Verschwinden der Schmerzen.

1.1.2.1.2.2.2 Parodontale Schmerzen zurückzuführen auf eine extraradikuläre endodontische Infektion

\section{Diagnostische Kriterien:}

A. Schmerzen, die die Kriterien für 1.1.2.1.2.2 Parodontale Schmerzen zurückzuführen auf eine endodontische Infektion erfüllen

B. Es wurde eine extraradikuläre Infektion ${ }^{1}$ im Umfeld eines Zahnes oder mehrerer Zähne diagnostiziert

C. Nicht besser erklärt durch eine andere ICOP-Diagnose

\section{Anmerkung:}

1. Die Infektion kann von Bakterien, Viren, Pilzen oder sonstigem ausgelöst sein.

Kommentar: Bei 1.1.2.1.2.2.2 Parodontale Schmerzen zurückzuführen auf eine extraradikuläre endodontische Infektion besiedeln die Infektionserreger, die die parodontale Entzündung verursachen, die äußere Wurzeloberfläche, periapikal oder in Verbindung mit den Mündungen von Seitenkanälen, beziehungsweise das periapikale Gewebe.

Eine extraradikuläre endodontische Infektion kann mit oder ohne eine interradikuläre Infektion auftreten. In beiden Fällen besiedeln die Mikroben die Außenseite des Foramen apicale sowie die Wurzeloberfläche und bilden einen Biofilm. Anaerobe Bakteriengattungen wie Actinomyceten und das Propionibakterium verfügen ebenfalls über die Fähigkeit, im periapikalen Gewebe etwas von der Wurzel entfernt Kolonien zu bilden, was mit verbleibenden Symptomen einschließlich Schmerzen nach einer Wurzelkanalbehandlung in Verbindung gebracht wurde.

Der Schmerz verschwindet üblicherweise nach erfolgreicher Desinfektion des Wurzelkanalsystems nicht. Die Bildgebung zeigt gelegentlich Zeichen einer externen apikalen Wurzelresorption.

1.1.2.1.3 Parodontale Schmerzen zurückzuführen auf eine parodontale Erkrankung

\section{Diagnostische Kriterien:}

A. Schmerzen, die die Kriterien für 1.1.2.1 Parodontale Schmerzen zurückzuführen auf eine Parodontitis sowie Kriterium C erfüllen

B. Es wurde eine Parodontitis ${ }^{1}$ diagnostiziert

C. Ein ursächlicher Zusammenhang ist aufgrund der anatomischen, funktionellen und/oder zeitlichen Verbindung plausibel $^{2}$

D. Nicht besser erklärt durch eine andere ICOP-Diagnose

\section{Anmerkungen:}

1. Die Erkrankung wird bei jeder Unterform spezifiziert.

2. Dieses Kriterium gilt für alle Unterformen. 
Kommentar: Parodontale Schmerzen aufgrund einer plaqueinduzierten Parodontitis können akuter oder chronischer Natur sein, und die Schmerzintensität reicht von leicht bis stark.

Die Erkrankung kann im Gebiss lokalisiert oder generalisiert sein. Eine Reihe intrinsischer (Diabetes, Schwangerschaft, Pubertät, Klimakterium) und extrinsischer (Rauchen, Medikamente, Mangelernährung [z. B. C-Avitaminose]) Faktoren gelten als Krankheitsmodifikatoren. Zudem können Medikamente, die bekanntermaßen mit einer Gingivahyperplasie einhergehen können (z. B. Phenytoin, Ciclosporin, Kalziumkanalblocker, Bisphosphonate und orale Kontrazeptiva), aufgrund von Schwierigkeiten mit der Aufrechterhaltung einer adäquaten Mundhygiene einen parodontalen Gewebeabbau begünstigen.

1.1.2.1.3.1 Parodontale Schmerzen zurückzuführen auf eine chronische Parodontitis

\section{Diagnostische Kriterien:}

A. Schmerzen, die die Kriterien für 1.1.2.1.3 Parodontale Schmerzen zurückzuführen auf eine parodontale Erkrankung erfüllen

B. Es wurde eine chronische Parodontitis diagnostiziert

C. Nicht besser erklärt durch eine andere ICOP-Diagnose

Kommentar: 1.1.2.1.3.1 Parodontale Schmerzen zurückzuführen auf eine chronische Parodontitis können in Verbindung mit einer erhöhten Zahnmobilität und schlechten Mundhygiene auftreten und sind üblicherweise leichter Natur. Die Schmerzen treten in der Regel nur bei Provokation auf und halten nicht an. In den meisten Fällen verläuft eine chronische Parodontitis nicht schmerzhaft, sondern kann bei einer entzündlichen Verschlimmerung schmerzhaft werden (siehe 1.1.2.1.3.5 Parodontale Schmerzen zurückzuführen auf einen parodontalen Abszess).

Eine chronische Parodontitis ist durch einen langsam progredienten Attachment-Verlust gekennzeichnet, mitunter mit Perioden schnellerer Progression. Das Nichtvorhandensein oder die leichte Ausprägung von Schmerzen wurde auf die primär chronisch-entzündlichen Zellinfiltrate im Umfeld der Infektionsquelle sowie eine funktionelle Drainage zurückgeführt.

1.1.2.1.3.2 Parodontale Schmerzen zurückzuführen auf eine aggressive Parodontitis

\section{Diagnostische Kriterien:}

A. Schmerzen, die die Kriterien für 1.1.2.1.3 Parodontale Schmerzen zurückzuführen auf eine parodontale Erkrankung erfüllen

B. Es wurde eine aggressive Parodontitis diagnostiziert

C. Nicht besser erklärt durch eine andere ICOP-Diagnose

Kommentar: 1.1.2.1.3.2 Parodontale Schmerzen zurückzuführen auf eine aggressive Parodontitis können in Verbindung mit einer erhöhten Zahnmobilität und schlechter Mundhygiene auftreten und sind üblicherweise leicht bis mittelstark. Die Schmerzen treten in der Regel nur bei Provokation auf und halten nicht an.

Eine aggressive Parodontitis ist von einem schnell progredienten Attachment-Verlust gekennzeichnet und mitunter von einem Beginn in frühen Jahren.
1.1.2.1.3.3 Parodontale Schmerzen zurückzuführen auf eine Parodontitis als Manifestation einer systemischen Erkrankung

\section{Diagnostische Kriterien:}

A. Schmerzen, die die Kriterien für 1.1.2.1.3 Parodontale Schmerzen zurückzuführen auf eine parodontale Erkrankung sowie Kriterium D erfüllen

B. Es wurde eine systemische Erkrankung ${ }^{1}$ diagnostiziert, die bekanntermaßen Parodontitis verursachen kann

C. Parodontitis ist als Manifestation der systemischen Erkrankung aufgetreten

D. Die Schmerzursache ist klinisch plausibel

E. Nicht besser erklärt durch eine andere ICOP-Diagnose

\section{Anmerkung:}

1. Die systemische Störung wird für jede Unterform spezifiziert.

Kommentar: Zusätzlich zu der häufigeren plaquebedingten Parodontitis manifestiert sich eine ganze Reihe systemischer Erkrankungen ebenfalls in Form einer Parodontitis. Die unten aufgeführten Erkrankungen gelten als ursächliche Faktoren für eine Parodontitis. Sie können zudem den Verlauf einer plaquebedingten Parodontitis von chronisch zu aggressiv verändern.

1.1.2.1.3.3 Parodontale Schmerzen zurückzuführen auf eine Parodontitis als Manifestation einer systemischen Erkrankung können in Verbindung mit einer erhöhten Zahnmobilität und schlechter Mundhygiene auftreten. Die Schmerzen sind in der Regel leicht bis mittelstark, treten nur bei Provokation auf und halten nicht an. Berichte über das Ausmaß, in dem Parodontitis als Manifestation einer systemischen Erkrankung mit Schmerz verbunden ist, sind in der Fachliteratur quasi nicht existent.

1.1.2.1.3.3.1 Parodontale Schmerzen zurückzuführen auf eine hämatologische Erkrankung

\section{Diagnostische Kriterien:}

A. Schmerzen, die die Kriterien für 1.1.2.1.3.3 Parodontale Schmerzen zurückzuführen auf eine Parodontitis als Manifestation einer systemischen Erkrankung erfüllen

B. Bei der systemischen Erkrankung handelt es sich um eine der folgenden:

1. Erworbene Neutropenie

2. Leukämie

3. Sonstige hämatologische Erkrankung, die bekanntermaßen Parodontitis verursachen kann

1.1.2.1.3.3.2 Parodontale Schmerzen zurückzuführen auf eine genetische Störung

\section{Diagnostische Kriterien:}

A. Schmerzen, die die Kriterien für 1.1.2.1.3.3 Parodontale Schmerzen zurückzuführen auf eine Parodontitis als Manifestation einer systemischen Erkrankung erfüllen

B. Bei der systemischen Erkrankung handelt es sich um eine der folgenden:

1. Familiäre und zyklische Neutropenie

2. Down-Syndrom

3. Leukozytenadhäsionsdefizienz-Syndrome

4. Papillon-Lefèvre-Syndrom 
5. Chediak-Higashi-Syndrom

6. Histiozytose-Syndrome

7. Glykogenspeicherkrankheit

8. Infantile hereditäre Agranulozytose

9. Cohen-Syndrom

10.Ehlers-Danlos-Syndrom (Typ IV und VIII)

11. Hypophosphatasie

12.Eine sonstige genetische Störung, die bekanntermaßen Parodontitis verursachen kann

1.1.2.1.3.3.3 Parodontale Schmerzen zurückzuführen auf eine nicht näher spezifizierte systemische Erkrankung

\section{Diagnostische Kriterien:}

A. Schmerzen, die die Kriterien für 1.1.2.1.3.3 Parodontale Schmerzen zurückzuführen auf eine Parodontitis als Manifestation einer systemischen Erkrankung erfüllen

B. Die systemische Erkrankung kann bekanntermaßen eine Parodontitis auslösen, ist aber weder hämatologischer noch genetischer Natur ${ }^{1}$

\section{Anmerkung:}

1. In Verbindung mit einer Parodontitis auftretende systemische Erkrankungen sind derzeit in der Fachliteratur nicht befriedigend beschrieben.

1.1.2.1.3.4 Parodontale Schmerzen zurückzuführen auf eine nekrotisierende ulzerative Parodontitis (NUP)

\section{Diagnostische Kriterien:}

A. Schmerzen, die die Kriterien für 1.1.2.1.3 Parodontale Schmerzen zurückzuführen auf eine parodontale Erkrankung sowie Kriterium C erfüllen

B. Es wurde eine nekrotisierende ulzerative Parodontitis diagnostiziert

C. Der Schmerz hat sich innerhalb von Stunden bis Tagen nach Auftreten der Ulzerationen entwickelt

D. Nicht besser erklärt durch eine andere ICOP-Diagnose

Kommentar: Die nekrotisierende ulzerative Parodontitis (NUP) ist eine seltene orale Infektion, eine schwerere Form einer nekrotisierenden (ulzerativen) Gingivitis, die - abgesehen davon, dass sie eine Zerstörung des Weichgewebes verursacht - auch einen Attachment-Verlust und Alveolarkammresorption mit sich bringt. Die beiden Krankheitsbilder werden oft unter „nekrotisierende parodontale Erkrankungen" (engl. Necrotizing periodontal diseases, NPD) zusammengefasst und gehen mit einer verminderten systemischen Resistenz und einer Immundysfunktion einher. $\mathrm{Zu}$ den prädisponierenden Faktoren gehören hohe Stressbelastung, Schlafmangel, Alkohol, Rauchen und HIV-Infektion.

1.1.2.1.3.4 Parodontale Schmerzen zurückzuführen auf eine nekrotisierende ulzerative Parodontitis (NUP) sind üblicherweise stark. Der Schmerz lässt sich durch physische Reize provozieren, die auf den betroffenen Zahn oder das umgebende Gewebe einwirken. Er tritt auch spontan auf.

Klinisch lassen sich nekrotische Weichgewebeläsionen und Attachment-Verlust beobachten.
1.1.2.1.3.5 Parodontale Schmerzen zurückzuführen auf einen parodontalen Abszess

\section{Diagnostische Kriterien:}

A. Schmerzen, die die Kriterien für 1.1.2.1.3 Parodontale Schmerzen zurückzuführen auf eine parodontale Erkrankung sowie Kriterium C erfüllen

B. Es wurde anhand von einem oder beiden der folgenden Punkte ein parodontaler Abszess diagnostiziert:

1. Klinische Zeichen einer akuten Entzündung ${ }^{1}$ und Attachment-Verlust ${ }^{2}$

2. Radiologische Nachweise einer marginalen und periradikulären Knochenresorption

C. Der Schmerz hat sich in einer engen zeitlichen Beziehung ${ }^{3} z u$ dem Abszess entwickelt

D. Nicht besser erklärt durch eine andere ICOP-Diagnose

\section{Anmerkungen:}

1. Zu den klinischen Zeichen gehören Schwellung, Rötung, Berührungsempfindlichkeit und/oder Vorliegen von Eiter.

2. Nachweis eines Attachment-Verlustes durch erhöhte Mobilität und/oder eine lokale tiefe Zahnfleischtasche.

3. Üblicherweise Stunden bis Tage vor Auftreten des Abszesses.

Kommentar: Ein parodontaler Abszess ist auf eine Exazerbation einer chronischen Parodontitis oder aggressiven Parodontitis zurückzuführen. Der hierdurch ausgelöste Schmerz ist in der Regel stark. Neben einer Schwellung finden sich noch weitere klinische Befunde wie Plaque und/oder Zahnsteinablagerungen auf der Wurzeloberfläche, gewöhnlich verbunden mit einer erhöhten Zahnbeweglichkeit und einer lokalen tiefen Zahnfleischtasche. Sofern nicht kurz zuvor eine Wurzelkanalbehandlung vorgenommen wurde, zeigt der Zahn üblicherweise eine vitale Pulpa. Die Bildgebung zeigt Zeichen einer marginalen und periradikuläre Knochenresorption, bei der die periapikale Region mitbetroffen sein kann, aber nicht muss.

Obwohl der Schmerz räumlich begrenzt ist, wird dieser oft auf andere orofaziale Regionen auf der gleichen Seite übertragen und/ oder strahlt dorthin aus, vor allem wenn der Schmerz stark ist. Der Schmerz lässt sich durch Klopfen oder Druckausübung auf den Zahn und/oder die benachbarte periapikale vestibuläre Region reproduzieren.

1.1.2.1.4 Parodontale Schmerzen zurückzuführen auf eine apikale und marginale Parodontitis aufgrund einer Kombination von endodontischer Infektion und Parodontitis

\section{Diagnostische Kriterien:}

A. Schmerzen, die die Kriterien für 1.1.2.1 Parodontale Schmerzen zurückzuführen auf eine Parodontitis sowie Kriterium C erfüllen

B. Es wurde beides von Folgendem diagnostiziert ${ }^{1}$ :

1. Partielle oder komplette Pulpanekrose, oder ein Zahn wurde zuvor wurzelkanalbehandelt

2. Parodontitis

C. Der ursächliche Zusammenhang ist auf der Basis anatomischer, funktioneller und/oder zeitlicher Beziehungen plausibel

D. Nicht besser erklärt durch eine andere ICOP-Diagnose 


\section{Anmerkung:}

1. Die Diagnose stützt sich auf klinische und radiologische Beobachtung.

Kommentar: Eine kombinierte endodontische und parodontale Läsion kann schmerzfrei sein. Sofern Schmerz vorliegt, ist dieser üblicherweise mäßig bis stark ausgeprägt, und sonstige klinische Befunde können unter anderem Anzeichen einer akuten Entzündung aufweisen (Schwellung, Rötung, Vorliegen von Eiter), Plaque und/oder Zahnsteinablagerung auf der Wurzeloberfläche, erhöhte Zahnbeweglichkeit und tiefe Zahnfleischtasche(n). Sofern zuvor keine Wurzelkanalbehandlung stattgefunden hat, zeigt der Zahn keinen oder keinen schlüssigen Nachweis einer vitalen Pulpa. Die Bildgebung zeigt eine marginale und periradikuläre Knochenresorption einschließlich der periapikalen Region.

Obwohl lokalisiert, wird der Schmerz häufig auf andere orofaziale Bereiche der gleichen Seite übertragen und/oder strahlt dorthin aus, vor allem wenn der Schmerz stark ist. Der Schmerz lässt sich durch Klopfen oder Druckausübung auf den Zahn und/oder die benachbarte periapikale vestibuläre Region reproduzieren.

1.1.2.1.5 Parodontale Schmerzen zurückzuführen auf eine infektiöse Periimplantitis

\section{Diagnostische Kriterien:}

A. Schmerzen, die die Kriterien für 1.1.2.1 Parodontale Schmerzen zurückzuführen auf eine Parodontitis sowie Kriterium C erfüllen, nur dass hier ein Implantat und nicht ein natürlicher Zahn betroffen ist

B. Es besteht ein klinischer ${ }^{1}$ und/oder radiologischer ${ }^{2}$ Nachweis einer infektiösen Periimplantitis

C. Ein ursächlicher Zusammenhang ist aufgrund der anatomischen, funktionellen und/oder zeitlichen Verbindung plausibel

D. Nicht besser erklärt durch eine andere ICOP-Diagnose

\section{Anmerkungen:}

1. Zum klinischen Nachweis gehörten Zeichen einer akuten Entzündung (Schwellung, Rötung, Vorliegen von Eiter) und/oder Attachment-Verlust (erhöhte Mobilität, tiefe Tasche).

2. Zum radiologischen Nachweis gehört eine Radioluzenz partiell oder komplett angrenzend an das Implantat.

Kommentar: Eine Entzündung um ein Zahnimplantat ist meist schmerzlos; sofern jedoch Schmerzen auftreten, sind diese üblicherweise mittelstark bis stark.

$\mathrm{Zu}$ den sonstigen klinischen Befunden können Plaque- und/ oder Zahnsteinablagerungen auf der Implantatoberfläche gehören. Die Bildgebung zeigt eine schlechte knöcherne Integration des Implantats und ergibt den Nachweis eines horizontalen marginalen Knochenabbaus oder einer lokalisierten periimplantären Knochenresorption.

Patienten mit 1.1.2.1.5 parodontalen Schmerzen zurückzuführen auf eine infektiöse Periimplantitis sind aller Wahrscheinlichkeit nach auch von 1.1.3 Gingivalen Schmerzen betroffen.
1.1.2.2 Parodontale Schmerzen zurückzuführen auf eine lokale nichtentzündliche Ursache

\section{Diagnostische Kriterien:}

A. Schmerzen, die die Kriterien für 1.1.2 Parodontale Schmerzen sowie Kriterium C erfüllen

B. Es wurde eine lokale nicht entzündliche Erkrankung diagnostiziert, die bekanntermaßen parodontale Schmerzen ${ }^{1}$ auslösen kann $^{2}$

C. Der ursächliche Zusammenhang mit dem Schmerz ist aufgrund der anatomischen und/oder zeitlichen Verbindung klinisch plausibel

D. Nicht besser erklärt durch eine andere ICOP-Diagnose

\section{Anmerkungen:}

1. Beispiele für derartige Erkrankungen sind eine parodontale Zyste oder ein Tumor.

2. Die Diagnose erfolgt durch Bildgebung sowie klinische und/ oder histologische Untersuchung.

Kommentar: 1.1.2.2 Parodontale Schmerzen zurückzuführen auf eine lokale nichtentzündliche Ursache sind gewöhnlich leicht bis mittelstark.

Parodontale Zysten, radikuläre Zysten und Tumore sind häufig asymptomatisch, doch nach einer Expansion können Symptome wie Schmerzen, lokalisierte Schwellung und Verdrängung eines Zahns oder mehrerer Zähne auftreten. In solchen Fällen wird der Schmerz gelegentlich durch externe mechanische Stimulation evoziert, etwa durch Beißen oder Kauen, und ist für Patienten üblicherweise leicht zu lokalisieren. Es kann auch zu Spontanschmerz kommen, der selten stark ist.

\subsubsection{Gingivale Schmerzen}

Beschreibung: Schmerzen, die durch eine Läsion oder Erkrankung verursacht werden, bei der die Gingiva beteiligt ist.

An anderer Stelle kodiert: Gingivale Schmerzen, die begleitend zu Erkrankungen auftreten, von denen primär andere Mundgewebe betroffen sind, werden in anderen Abschnitten klassifiziert: gingivale Schmerzen zurückzuführen auf eine Alveolitis sicca (trockenes Zahnfach) siehe 1.2.3.5 Schmerzen im Kieferknochen zurückzuführen aufeine Therapie; gingivale Schmerzen zurückzuführen auf Parodontitis siehe 1.1.2.1 Parodontale Schmerzen zurückzuführen auf eine Parodontitis (parodontale Entzündung); gingivale Schmerzen zurückzuführen auf eine apikale Parodontitis siehe 1.1.2.1.2 Parodontale Schmerzen zurückzuführen auf eine apikale Parodontitis aufgrund einer endodontischen Erkrankung; palatinale gingivale Schmerzen zurückzuführen auf eine akute nekrotisierende Sialadenitis siehe 1.2.2.2.1 Schmerzen der Speicheldrüse zurückzuführen auf eine bakterielle Infektion.

Gingivale Schmerzen zurückzuführen auf eine Neuropathie siehe 4.1 Schmerzen zurückzuführen auf eine Läsion oder Erkrankung des N. trigeminus. Triggerzonen einer 4.1.1 Trigeminusneuralgie können im Zahnfleisch lokalisiert sein, und eine leichte Berührung ruft die charakteristischen heftigen paroxysmalen Schmerzattacken hervor, die das gesamte Dermatom betreffen, das dem betroffenen Nervenast entspricht. Infolgedessen kann es Patienten 
unmöglich sein, in dieser Region einen Zahnersatz zu tragen. Gingivale Schmerzen können auch als Teil des frühen klinischen Erscheinungsbildes einer 4.1.1 Trigeminusneuralgie auftreten, der diffuse tiefe „Prä-Trigeminusneuralgie-Schmerz", der mitunter dem Beginn der charakteristischen paroxysmalen Schmerzen vorangeht.

Zur peripheren Neuropathie begleitet von gingivalen Schmerzen siehe 4.1.2 Andere neuropathische Schmerzen im Innervationsgebiet des N. trigeminus.

$\mathrm{Zu}$ idiopathischen gingivalen Schmerzen siehe 6. Idiopathische orofaziale Schmerzen. Das Symptom des brennenden Mundes (engl. Burning mouth syndrome, BMS) kann auch das Zahnfleisch in Mitleidenschaft ziehen und tritt dabei als lokalisierte oder auch auf ein größeres Areal verteilte gingivale Schmerzen auf (siehe 6.1 Syndrom des brennenden Mundes). Anhaltende idiopathische dentoalveoläre Schmerzen (engl. Persistent idiopathic dentoalveolar pain, PIDAP) sind häufig von lokalisiertem Schmerz im Zahnfleisch (siehe 6.3 Anhaltende idiopathische dentoalveoläre Schmerzen) begleitet.

Gesondert zu beachten sind Patienten mit gingivalen Schmerzen in Verbindung mit Fibromyalgiesyndrom (engl. Chronic widespread pain, CWP) oder sonstigen multiplen Schmerzerkrankungen, die auf eine zentrale Sensibilisierung oder andere Mechanismen zurückzuführen sein mögen.

\section{Diagnostische Kriterien:}

A. Der gingivale Schmerz ${ }^{1}$ erfüllt Kriterium C

B. Es wurde klinisch, laborchemisch, mittels Bildgebung und/ oder anamnestisch eine Läsion oder Erkrankung des Zahnfleischgewebes nachgewiesen, die bekanntermaßen Schmerzen auslösen kann

C. Der Nachweis des ursächlichen Zusammenhangs besteht aufgrund von mindestens zwei der folgenden Punkte:

1. Die Lokalisation des Schmerzes entspricht dem/den Situs der Läsion oder Erkrankung ${ }^{1}$

2. Der Schmerz entstand in einem zeitlichen Zusammenhang mit dem erstmaligen Auftreten oder Beginn der Läsion oder Erkrankung

3. Der Schmerz verschlimmert sich durch Manipulation des betroffenen Zahnfleischgewebes

D. Nicht besser erklärt durch eine andere ICOP-Diagnose

\section{Anmerkung:}

1. Der Schmerz kann auch auf andere ipsilaterale orofaziale Areale übertragen werden und/oder ausstrahlen.

\subsubsection{Gingivale Schmerzen zurückzuführen auf eine Gingivitis (Zahnfleischentzündung)}

\section{Diagnostische Kriterien:}

A. Schmerzen, die die Kriterien für 1.1.3 Gingivale Schmerzen erfüllen

B. Es wurde eine Gingivitis ${ }^{1}$ diagnostiziert $^{2}$

C. Nicht besser erklärt durch eine andere ICOP-Diagnose.

\section{Anmerkungen:}

1. Gingivitis infolge eines Traumas, einer Infektion oder systemischen Erkrankung wird innerhalb jeder Unterform spezifiziert.
2. Die Diagnose erfolgt durch klinische Beobachtung von Entzündungszeichen (Schwellung, Rötung und Bluten) im Zahnfleisch.

Kommentar: Eine Gingivitis kann auf eine Infektion aufgrund spezifischer oder nichtspezifischer Mikroorganismen, ein Trauma (physischer, thermischer, bestrahlungsbedingter oder chemischer Art), eine Autoimmunreaktion oder eine allergische Reaktion zurückgehen.

\subsection{Gingivale Schmerzen zurückzuführen auf ein Trauma} Diagnostische Kriterien:

A. Schmerzen, die die Kriterien für 1.1.3.1 Gingivale Schmerzen zurückzuführen auf eine Gingivitis sowie Kriterium C erfüllen

B. Es ist ein Trauma ${ }^{1}$ oder eine Verletzung ${ }^{2}$ eingetreten, das/die das Zahnfleischgewebe betrifft

C. Beide der folgenden Punkte sind erfüllt:

1. Die Lokalisation des Schmerzes entspricht dem traumatisierten oder verletzten Gewebe

2. Der Schmerz hat sich innerhalb von Minuten bis Tagen nach dem Trauma oder der Verletzung entwickelt

D. Nicht besser erklärt durch eine andere ICOP-Diagnose

\section{Anmerkungen:}

1. Das Trauma kann mechanischer, thermischer oder chemischer Art sein.

2. Die Verletzung kann unfallbedingt oder nicht unfallbedingt sein, von anderen zugefügt sein oder auf Selbstverletzung zurückgehen bzw. iatrogen sein.

Kommentar: Eine traumatische Verletzung des Zahnfleischgewebes verursacht eine akute Entzündung und kann in unterschiedlichem Umfang schmerzhaft sein. Eine traumatische Ulzeration des Zahnfleischs kann akuter oder chronischer Natur sein, wobei Letzteres diagnostisch aufgrund der zugrunde liegenden Fibrose und dem klinischen Erscheinungsbild einer neuroplastischen Induration eine größere Herausforderung darstellt.

1.1.3.1.1 Gingivale Schmerzen zurückzuführen auf ein Trauma können leicht bis stark sein und verschlimmern sich durch mechanische Provokation des Zahnfleischs. Es kann Spontanschmerz auftreten.

Zu den Ursachen von 1.1.3.1.1 Gingivalen Schmerzen zurückzuführen auf ein Trauma gehören unfallbedingte Zahnverletzungen, aber auch Mikrotraumen, wie sie zum Beispiel beim Verzehr oder Trinken von zu heißen Speisen oder Getränken, infolge einer Zahnbehandlung oder eines Traumas durch Zähneputzen oder den Gebrauch von Zahnseide oder anderen Hilfsmitteln zur Reinigung des Zahnzwischenraums entstehen. Eine gründliche klinische Anamnese macht Kliniker oft auf eine traumatische Ätiologie oder Verbrennungen durch zu warmes Essen oder chemische Stoffe aufmerksam. Eine nähere Untersuchung mag den ursächlichen Faktor offenbaren, etwa einen scharfkantigen abgebrochenen Zahn oder eine entsprechende Zahnrestauration oder ein schlechtsitzender Zahnersatz. Eine Ulzeration aufgrund einer mittels Spritze verabreichten örtlichen Betäubung tritt am häufigsten im harten Gaumen auf, das kombinierte Ergebnis von Druck und ischämischer 
Nekrose. Ein schlechtsitzender Zahnersatz kann schmerzhafte UIzerationen verursachen. Auch ein Zahnhochstand oder Parafunktionen können ein lokales okklusales Zahnfleischtrauma mit hieraus resultierender Entzündung und Schmerzen verursachen. latrogene gingivale Schädigungen treten während eines Großteils zahnchirurgischer Eingriffe auf, etwa bei Extraktion, chirurgischen Eingriffen am Zahnfleisch oder bei der Parodontalchirurgie oder Füllungstherapie. Verätzungen können in Verbindung mit einem Missbrauch antientzündlicher Tabletten oder aufgrund einer Zahnbehandlung auftreten. In seltenen Fällen kann Selbstschädigung Ursache eines Zahnfleischtraumas sein.

Auch ein Zahntrauma kann Schmerzen durch eine Zahnfleischentzündung verursachen (siehe 1.1.2.1.1 Parodontale Schmerzen zurückzuführen auf eine traumatisch bedingte parodontale Entzündung).

1.1.3.1.2 Gingivale Schmerzen zurückzuführen auf eine Infektion Diagnostische Kriterien:

A. Schmerzen, die die Kriterien für 1.1.3.1 Gingivale Schmerzen zurückzuführen auf eine Gingivitis sowie Kriterium C erfüllen

B. Es wurde eine Infektion' des Zahnfleischgewebes diagnostiziert $^{2}$

C. Der Schmerz hat sich in einem engen zeitlichen Zusammenhang mit der Infektion entwickelt oder führte zu ihrer Entdeckung

D. Nicht besser erklärt durch eine andere ICOP-Diagnose

\section{Anmerkungen:}

1. Die Infektion kann durch Bakterien, Viren oder Pilze verursacht sein, wie bei der jeweiligen Unterform spezifiziert.

2. Die Diagnose stützt sich auf anamnestische Informationen, klinische Beobachtungen und/oder mikrobiologische Analyse.

Kommentar: Eine Infektion des Zahnfleischgewebes verursacht eine akute Entzündung und kann in unterschiedlichem Ausmaß schmerzhaft sein: Der Schmerz kann leicht bis stark sein und verschlimmert sich durch mechanische Provokation des Zahnfleischs. Es kann Spontanschmerz auftreten.

Eine erworbene oder angeborene Immunsuppression kann das Risiko einer Zahnfleischinfektion erhöhen. Immunsuppressiv behandelte Patienten können eine Vielzahl opportunistischer Infektionen entwickeln, einschließlich einer pseudomembranösen Candidose und anderer Pilz- und Virusinfektionen. Tumornekrosefaktor(TNF)-a-Therapie erhöht das Risiko einer Tuberkulose (TB). Patienten unter Medikation mit Infliximab und Adalimumab mit kombinierter immunmodulatorischer Stufentherapie können verstärkt gefährdet sein, TB, Histoplasmose und/oder Coccidiomykose-Infektionen zu entwickeln. Antirheumatische Medikamente wie Methotrexat, Abatacept und Alefacept zeigten ein erhöhtes Risiko von Herpes-simplex- und Herpes-Zoster-Infektionen sowie TB.

1.1.3.1.2 Gingivale Schmerzen zurückzuführen auf eine Infektion werden nach dem ursächlichen Mikroorganismus in Unterkategorien untergliedert.
1.1.3.1.2.1 Gingivale Schmerzen zurückzuführen auf eine bakterielle Infektion

\section{Diagnostische Kriterien:}

A. Schmerzen, die die Kriterien für 1.1.3.1.2 Gingivale Schmerzen zurückzuführen auf eine Infektion erfüllen

B. Die Infektion ist bakterieller Art

Kommentar: Bakterielle Infektionen sind die häufigsten oralen Infektionen und gingivale Schmerzen können mit einer zugrunde liegenden pathologischen Veränderung der Zähne zusammenhängen, etwa einer parodontalen Infektion oder endodontischen Infektionen, die sich als Schwellung, Entzündung und Schmerzen des darüber liegenden Zahnfleischs zeigen können.

Eine akute nekrotisierende ulzerative Gingivitis (ANUG; oder nekrotisierende ulzerative Gingivitis [NUG], nekrotisierende ulzerative Parodontitis [NUP] oder nekrotisierende ulzerative Stomatitis [NUS]) ist eine opportunistische gingivale Infektion, die bei unterernährten Kindern, jungen Erwachsenen und Patienten mit Immundefizit durch eine Palette von Bakterien ausgelöst wird. NUG ist oft das sich anfänglich präsentierende Krankheitsbild, dann fortschreitend zu NUP, NUS und am Ende Noma (eine Form von Gangrän, die das Gesicht befällt). Nekrose und Ulzeration der Interdentalpapille, quälende Schmerzen, starker Mundgeruch, regionale Lymphadenopathie, Unwohlsein und Fieber unterscheiden diese Form von Ulzeration von anderen.

Eine schmerzauslösende Perikoronitis (Entzündung um eine Zahnkrone) steht am häufigsten mit nur teilweise durchgebrochenen Weisheitszähnen in Verbindung. Beim Durchbrechen anderer Zähne kann, sowohl bezogen auf das bleibende wie auch das Milchgebiss, eine leichte Perikoronitis entstehen. Ist der Zahn impaktiert und kann nicht vollständig durchbrechen, kann eine Dauer- oder wiederkehrende Infektion entstehen. Die Schmerzen sind Folge der Immunentzündungsreaktion des Individuums auf im Plaque-Biofilm angesiedelten anaeroben Bakterien, die sich von partiell mit Weichgewebe bedeckten Weisheitszähnen nicht abstreifen lassen.

1.1.3.1.2.2 Gingivale Schmerzen zurückzuführen auf eine Virusinfektion

\section{Diagnostische Kriterien:}

A. Schmerzen, die die Kriterien für 1.1.3.1.2 Gingivale Schmerzen zurückzuführen auf eine Infektion erfüllen

B. Die Infektion ist viraler Art

Kommentar: Das infizierte Zahnfleischgewebe kann oft ulzeriert oder schmerzhaft bei Berührung sein. Starke lokale Schmerzen sind oft mit dem Verzehr oder Trinken säurehaltiger, heißer oder kalter Speisen oder Getränke verbunden, was dazu führen kann, dass die Person außerstande ist, Nahrung oder Getränke zu sich zu nehmen, und dehydriert.

Das Herpes-simplex-Virus (HSV) ist das häufigste Virus, das die Mundschleimhaut befällt. Gingivostomatitis herpetica (Mundfäule), die primäre HSV-1-Infektion, befällt meistens Kinder und äußert sich entweder als eine asymptomatische Infektion oder mit Schleimhautbläschen, gefolgt von einer schmerzhaften UIzeration, die sowohl keratinisiertes als auch nichtkeratinisiertes 
Schleimhaut- und Zahnfleischgewebe betrifft. Erwachsene mit einer primären Infektion erleiden eine symptomatische herpetische Pharyngotonsillitis, eingangs als Bläschen, die schnell in flache UIzerationen zerfallen.

Zu den sonstigen Virusinfektionen des Zahnfleischgewebes gehören Infektionen mit dem Varicella-Zoster-Virus (VZV), dem humanen Papillomvirus (HPV), dem Zytomegalievirus (ZMV), Coxsackieviren und HIV-Infektionen.

1.1.3.1.2.3 Gingivale Schmerzen zurückzuführen auf eine Pilzinfektion

\section{Diagnostische Kriterien:}

A. Schmerzen, die die Kriterien für 1.1.3.1.2 Gingivale Schmerzen zurückzuführen auf eine Infektion erfüllen

B. Es handelt sich um eine Pilzinfektion

Kommentar: Gingivale Schmerzen in Verbindung mit einer Pilzinfektion sind wahrscheinlich selten, und es mangelt im Grunde an Berichten in der Fachliteratur. Die schmerzhaften Manifestationen einer oralen Pilzinfektion betreffen üblicherweise die Mundschleimhaut.

1.1.3.1.3 Gingivale Schmerzen zurückzuführen auf eine Autoimmunreaktion

\section{Diagnostische Kriterien:}

A. Schmerzen, die die Kriterien für 1.1.3.1 Gingivale Schmerzen zurückzuführen auf eine Gingivitis sowie Kriterium C erfüllen

B. Es wurde eine Autoimmunerkrankung oder Störung diagnostiziert, die bekanntermaßen gingivale Schmerzen ${ }^{1}$ verursachen kann

C. Der ursächliche Zusammenhang mit dem Schmerz ist klinisch plausibel

D. Nicht besser erklärt durch eine andere ICOP-Diagnose

\section{Anmerkung:}

1. Beispiele sind Schleimhautpemphigoid, Sjögren-Syndrom und Pemphigus.

Kommentar: 1.1.3.1.3 Gingivale Schmerzen zurückzuführen auf eine Autoimmunreaktion können leicht bis stark sein und verschlimmern sich durch mechanische Provokation des Zahnfleischs. Es kann Spontanschmerz auftreten.

Zustände durch diverse dermatologische immunvermittelte vesikulär-ulzerative Läsionen können sich mit Beteiligung der Mundschleimhaut zeigen, entweder begleitend zu den Hautveränderungen, als Anfangs- oder mitunter einzige klinische Manifestation.

Das Schleimhautpemphigoid (engl. Mucous membrane pemphigoid, MMP) ist eine häufige systemische blasenbildende Autoimmunerkrankung mit vorzugsweiser Beteiligung der Schleimhäute. Die Antikörper richten sich gegen die Keratinozytenproteine zur Adhäsion der Bindegewebsmatrix oder Hemidesmosomen (BP180 und Laminin-332), was bewirkt, dass das Epithel sich von seinem darunterliegenden Bindegewebsbett löst. Die subepitheliale Natur des Adhäsionsverlusts führt zu Blasen mit dünnem Dach, die bei der Untersuchung noch intakt sein können. Das Aufplatzen der Blasen hinterlässt großfächige ulzerative Läsionen ohne Epi- thel, bedeckt von gelblich-weißem Schorf. Häufig findet sich eine desquamative Gingivitis (erythematöse und beanspruchte Gingiva mit Zerstörung von Epithelzellen).

Das Sjögren-Syndrom ist eine systemische Autoimmunerkrankung, die oft gleichzeitig mit anderen systemischen Bindegewebs- oder organspezifischen Autoimmunerkrankungen auftritt. Die Verbindung ist gut beschrieben für systemischen Lupus erythematodes und rheumatoide Arthritis. Das Zahnfleischgewebe kann von trockenen Nahrungsmitteln abgescheuert und sogar eingeschnitten werden, und es kann wund werden. Das Vorliegen des Sjögren-Syndroms beeinflusst in einem bestimmten Umfang die Expression der anderen Autoimmunerkrankungen, zum Beispiel durch Erhöhung der Müdigkeit und des Lymphomrisikos.

Pemphigus, eine Gruppe immunvermittelter subepithelialer bullöser Dermatosen, wird durch Autoantikörper vermittelt, die sich gegen die Proteine der Keratinozytenadhäsion richten (Desmosomen) und eine Akantholyse bewirken. Pemphigus vulgaris betrifft am häufigsten die Mundhöhle, wobei sich die Autoantikörper in erster Linie gegen Desmoglein-1 und 3 (mukokutane Formen) oder nur 3 (mukosale Formen) richten. Gingivale Schmerzen aufgrund von Pemphigus sind selten, da die Erkrankung meist die Mundschleimhaut betrifft.

1.1.3.1.4 Gingivale Schmerzen zurückzuführen auf eine Überempfindlichkeit oder allergische Reaktion

\section{Diagnostische Kriterien:}

A. Schmerzen, die die Kriterien für 1.1.3.1 Gingivale Schmerzen zurückzuführen auf eine Gingivitis sowie Kriterium C erfüllen

B. Eine Überempfindlichkeit oder allergische Reaktion im Zahnfleischgewebe ${ }^{1}$ ist aufgetreten

C. Der Schmerz hat sich in zeitlichem Zusammenhang mit der Überempfindlichkeit oder allergischen Reaktion entwickelt oder zu ihrer Entdeckung geführt

D. Nicht besser erklärt durch eine andere ICOP-Diagnose

\section{Anmerkung:}

1. Die Überempfindlichkeit oder allergische Reaktion kann in Verbindung mit Dentalwerkstoffen auftreten (etwa temporärem oder permanentem Zahnrestaurations- oder Abformmaterial), einem Mundhygieneprodukt, einem topischen oder systemischen Medikament, einem Nahrungsmittel oder Nahrungsmittelzusatz oder einem anderen Faktor.

Kommentar: 1.1.3.1.4 Gingivale Schmerzen zurückzuführen auf eine Überempfindlichkeit oder allergische Reaktion können leicht bis stark sein und verschlimmern sich durch mechanische Provokation des Zahnfleischs. Es kann ein Spontanschmerz auftreten.

Allergische Reaktionen und Überempfindlichkeitsreaktionen der Mundschleimhaut sind weniger weit verbreitet als kutane Reaktionen dieser Art, vermutlich aufgrund der Verdünnung des Allergens und des kontinuierlichen Mundspüleffekts in Verbindung mit dem normalen Speichelfluss. Läsionen können sich als ein unspezifisches Gewebsödem, Erythem, als Rissbildung, Ulzeration, hyperkeratotische weiße Plaques oder Desquamation der Mundschleimhaut manifestieren. 
Üblicherweise lässt sich ein zeitlicher oder räumlicher Zusammenhang mit einer Substanz ermitteln, die solche Reaktionen auslöst. Im Falle einer arzneimittelassoziierten Überempfindlichkeit können lange Zeit nach Einleitung der Medikamentengabe Läsionen auftreten, und diese können noch Monate nach Absetzen des Medikaments bestehen bleiben, was Diagnose und Management erschwert.

Eine Überempfindlichkeitsreaktion auf ein systemisches Medikament oder eine Substanz, die entsprechende Reaktionen auslöst und mit der ein direkter Kontakt besteht, kann zu klinischen und histologischen Merkmalen führen, die an einen Lichen ruber planus erinnern. Begrifflich wird diese unter orale lichenoide Arzneimittelreaktionen (engl. Oral lichenoid drug reaction, OLDR) beziehungsweise orale lichenoide Kontaktläsion (engl. Oral lichenoid contact lesion, $\mathrm{OLCL}$ ) gefasst, und beide können sich mit erheblicher Ulzeration manifestieren, üblicherweise mit Erythemen und weißlicher Streifung an der Peripherie der Ulzeration. Oft ist an einer OLCL Amalgam beteiligt, bestätigt durch einen Patch-Test auf Überempfindlichkeit gegen Quecksilber oder Amalgam. OLDR begegnet man mit einer gewissen Häufigkeit bei mit Angiotensinkonvertierendes-Enzym-Hemmern (ACE-Hemmern), nichtsteroidalen Entzündungshemmern (NSAP) und oralen Antidiabetika behandelten Patienten.

Potenzielle Arzneimittelwirkungen, die orale mukogingivale Reaktionen auslösen, wurden gut zusammengefasst. Ein fixes Arzneimittelexanthem (engl. Fixed drug eruption, FDE) ist eine Form von Überempfindlichkeitsreaktion, die aufgrund ihrer Gebundenheit an eine bestimmte anatomische Stelle auffällig ist und in Verbindung mit NSAR und anderen Oxicamen, Gabapentin, Fuconazol sowie systemischen antibakteriellen und antimykotischen Medikamenten auftritt. Ein fixes Arzneimittelexanthem sollte in solchen Fällen in Betracht gezogen werden, in denen ein zeitlicher Zusammenhang mit der Einnahme des Medikaments besteht. Der Nachweis kann durch Patch-Test oder orale Provokationstests erbracht werden; das Management erfolgt durch Meiden des Medikaments oder Ersatz durch ein anderes, während die akuten Läsionen mit topischen oder systemischen Steroiden behandelt werden können.

In seltenen Fällen kommt es zu einer allergischen Kontaktstomatitis: einer Form von Mukositis, die in Verbindung mit Zahnabdruckmaterialien, Zahnrestaurationsmaterialien, topischer Benzocain-Anwendung und - durchaus häufiger - Zimt in Zahncremes, Mundspülungen und Kaugummi auftritt. Das Erscheinungsbild der Läsionen kann in gemischten roten und weißen Flecken mit Ulzeration, Schwellung der Wangen und Desquamation bestehen, die auf den Lippen, den Wangen, der Zunge und dem Zahnfleisch als lokalisierte oder breit gestreute Läsionen zutage treten.

Eine arzneimittelinduzierte Fibrose mit epithelialer Hyperplasie oder fibrovaskulärer Hyperplasie kann gelegentlich mit schmerzhaften Manifestationen einhergehen, vermutlich aufgrund der zugrundeliegenden parodontalen Infektion durch Schwierigkeiten mit der Mundhygiene in Verbindung mit diesen Krankheitsbildern.
1.1.3.1.5 Gingivale Schmerzen zurückzuführen auf eine Zahnfleischentzündung aufgrund einer anderen Ursache

\section{Diagnostische Kriterien:}

A. Schmerzen, die die Kriterien für 1.1.3.1 Gingivale Schmerzen zurückzuführen auf eine Gingivitis sowie Kriterium C erfüllen

B. Es wurde eine Erkrankung diagnostiziert, die bekanntermaßen eine Gingivitis auslösen kann, jedoch eine andere als unter 1.1.3.1.1 bis 1.1.3.1.4 ${ }^{1}$ genannt

C. Der Schmerz hat sich in einem engen zeitlichen Zusammenhang mit der Störung entwickelt oder führte zu ihrer Entdeckung

D. Nicht besser erklärt durch eine andere ICOP-Diagnose

\section{Anmerkung:}

1. Zu Störungen dieser Art gehören endokrine Störungen oder Veränderungen, Ernährungsdefizite, hämatologische Erkrankungen, gastrointestinale und dermatologische Erkrankungen, arzneimittelinduzierte Störungen (nicht zurückzuführen auf eine Überempfindlichkeit oder Allergie) sowie genetische Störungen.

Kommentar: 1.1.3.1.5 Gingivale Schmerzen zurückzuführen auf eine Zahnfleischentzündung aufgrund einer anderen Ursache können leicht bis stark sein und verschlimmern sich durch mechanische Provokation des Zahnfleischs. Es kann Spontanschmerz auftreten.

Eine Entzündung des Zahnfleischgewebes kann in Verbindung mit einer Erkrankung, einer Störung oder einem Zustand systemischer Art auftreten oder mit der Behandlung dieser Erkrankungen oder Störungsbilder verbunden sein.

Eine Veränderung der physiologischen Voraussetzungen, wie etwa durch Schwangerschaft und Wechseljahre, kann endokrine Veränderungen auslösen, die sich als gingivale Beschwerden und Schmerzen manifestieren. Zu den systemischen Erkrankungen, die ursächlich für eine Gingivitis sein können, gehören endokrine Erkrankungen (Schilddrüsenunterfunktion, Diabetes mellitus), Mangelernährung (Eisen-, Vitamin-B-Komplex-, Zink-Mangel), Anämie, gastrointestinale Störungen (gastroösophageale Refluxkrankheit) sowie arzneimittelinduzierte und genetische Störungen.

Eine Epulis ist eine hyperplastische, nichtneoplastische Veränderung, die primär auf das Zahnfleischgewebe zurückgeht. Es treten mehrere histologische Typen auf, von denen der während der Schwangerschaft vorherrschende Typus der granulomatöse ist, eine Form des pyogenen Granuloms. Es kommt hauptsächlich zu einem verstärkten Wachstum von Kapillargefäßen und einer Endothelproliferation. Dieser "Schwangerschaftstumor", wie er gelegentlich genannt wird, tritt in der Regel während des dritten Schwangerschaftsdrittels im vorderen Teil des Oberkiefers auf. Die Läsion verursacht üblicherweise, abgesehen von ihrem bloßen Vorhandensein, keine Symptome, kann jedoch aufgrund einer Beeinträchtigung der Okklusion oder Abnutzung des Gebisses Schmerzen nach sich ziehen. Ätiologische Faktoren sind ungenügende Mundhygiene, die zu einer chronischen Gingivitis führt, sowie ein hoher gingivaler Spiegel an aktivem Progesteron, dessen Wirkmechanismus bislang noch nicht näher definiert ist.

Eine antineoplastische therapieinduzierte Mukositis in Verbindung mit Chemotherapie und Bestrahlung befällt hauptsächlich 
die Mundschleimhaut (siehe 1.2.1.1.1.3 Schmerzen in der Mundschleimhaut zurückzuführen auf eine Bestrahlung oder Chemotherapie), kann aber auch das Zahnfleisch befallen und gingivale Schmerzen verursachen.

Gutartige hyperplastische Läsionen oder Tumoren unter Beteiligung des Zahnfleischs gehen üblicherweise nicht direkt mit Schmerzen einher, können jedoch schmerzhaft werden, wenn eine Traumatisierung und/oder Infektion aufgrund einer Beeinträchtigung der Okklusion oder des Zahnersatzes auftritt (siehe 1.1.3.1.1 Gingivale Schmerzen zurückzuführen auf ein Trauma und 1.1.3.1.2 Gingivale Schmerzen zurückzuführen auf eine Infektion).

\subsubsection{Gingivale Schmerzen zurückzuführen auf eine maligne Läsion Diagnostische Kriterien:}

A. Schmerzen, die die Kriterien für 1.1.3 Gingivale Schmerzen sowie Kriterium C erfüllen

B. Es wurde eine maligne Läsion des Zahnfleischgewebes diagnostiziert

C. Die Schmerzursache ist auf Grundlage des anatomischen und/ oder zeitlichen Zusammenhangs plausibel

D. Nicht besser erklärt durch eine andere ICOP-Diagnose

Kommentar: Das Zahnfleisch kann von einer ganzen Palette primärer wie auch metastasierender Malignome betroffen sein, die sich allesamt als unspezifische Geschwüre manifestieren. Am häufigsten kommen Plattenepithelkarzinome (engl. Oral squamous cell carcinoma, OSCC) vor. Diese manifestieren sich oft als Ulzeration mit klinischer Induration, Fixierung am darunterliegenden Gewebe, eingerollten exophytischen Rändern sowie Schmerzen und/ oder Taubheit.

1.1.3.2 Gingivale Schmerzen zurückzuführen auf eine maligne Läsion können leicht bis stark sein und werden durch mechanische Provokation des Zahnfleischs verschlimmert. Es können Spontanschmerzen auftreten.

1.2 Schmerzen der Mundschleimhaut, der Speicheldrüse und des Kieferknochens

Beschreibung: Schmerzen, die durch eine Läsion oder Störung verursacht werden, von der das nichtdentale orale und periorale Gewebe betroffen ist: Mundschleimhaut, Speicheldrüsen und Kieferknochen.

An anderer Stelle kodiert: Zu Schmerzen mit Ursprung in der orofazialen Muskulatur siehe 2. Myofaszial-orofaziale Schmerzen. Zu Schmerzen mit Ursprung im Kiefergelenk siehe 3. Kiefergelenksschmerzen.

\subsubsection{Schmerzen in der Mundschleimhaut}

Beschreibung: Schmerzen, die durch eine Erkrankung oder Störung verursacht werden, die die Mundschleimhaut betrifft.

An anderer Stelle kodiert: Zu Schmerzen in der Mundschleimhaut zurückzuführen auf eine Neuropathie siehe 4.1 Schmerzen zurückzuführen auf eine Läsion oder Erkrankung des N. trigeminus. (Schmerzen in der Mundschleimhaut können als Bestandteil des frühen klinischen Erscheinungsbildes einer 4.1.1 Trigeminusneuralgie auftreten: der diffuse tiefe „Prä-Trigeminusneuralgie-Schmerz", der mitunter dem Einsetzen des charakteristischen paroxysmalen Schmerzes vorausgeht. Die Triggerzonen einer 4.1.1 Trigeminusneuralgie können in der Mundschleimhaut lokalisiert sein, und leichte Berührung ruft die typischen intensiven paroxysmalen Schmerzattacken hervor, die das gesamte Dermatom erfassen, das dem betroffenen Nervenast entspricht.)

Zur peripheren Neuropathie in Zusammenhang mit Schmerzen in der Mundschleimhaut siehe 4.1.2 Andere neuropathische Schmerzen im Innervationsgebiet des $N$. trigeminus. Zu idiopathischen Schmerzen in der Mundschleimhaut siehe 6. Idiopathische orofaziale Schmerzen. Zum Syndrom des brennenden Mundes (engl. Burning mouth syndrome, BMS), das sich als lokalisierter oder breiter gestreuter Schmerz in der Mundschleimhaut manifestiert siehe 6.1 Syndrom des brennenden Mundes.

$\mathrm{Zu}$ anhaltenden idiopathischen dentoalveolären Schmerzen (PIDAP), mitunter in Verbindung mit lokalisierten Schmerzen in der benachbarten Mundschleimhaut siehe 6.3 Anhaltende idiopathische dentoalveoläre Schmerzen.

Gesondert zu beachten sind Patienten mit Schmerzen in der Mundschleimhaut in Verbindung mit einem Fibromyalgiesyndrom (engl. Chronic widespread pain, CWP) oder sonstigen multiplen Schmerzerkrankungen, die auf eine zentrale Sensibilisierung oder andere Mechanismen zurückzuführen sein mögen.

\section{Diagnostische Kriterien:}

A. Jeder Schmerz in der Mundschleimhaut ${ }^{1}$, der Kriterium C erfüllt

B. Klinisch, laborchemisch, mittels Bildgebung und/oder anamnestisch wurde eine Läsion oder Erkrankung des Mundschleimhautgewebes nachgewiesen, die bekanntermaßen Schmerzen auslösen kann²

C. Der ursächliche Zusammenhang ist durch alle folgenden Punkte belegt:

1. Die Lokalisation des Schmerzes entspricht dem/den Situs der Läsion oder Erkrankung ${ }^{1}$

2. Die Schmerzentstehung steht in zeitlichem Zusammenhang mit dem Auftreten oder Beginn der Läsion oder Erkrankung

3. Der Schmerz verschlimmert sich bei Manipulation der betroffenen Mundschleimhaut

D. Nicht besser erklärt durch eine andere ICOP-Diagnose

Es wurde eine Läsion, eine Erkrankung oder ein Trauma² nachgewiesen, die/das bekanntermaßen parodontale Schmerzen auslösen kann.

\section{Anmerkungen:}

1. Der Schmerz kann zu anderen ipsilateralen orofazialen Stellen übertragen werden und/oder ausstrahlen.

2. Die Läsion oder Erkrankung wird bei jeder Unterform spezifiziert.

Kommentar: Schmerzen, von denen die Mundschleimhaut betroffen ist, können einen lokalen oder einen weiter entfernten Aus- 
gangspunkt haben. Kennzeichnend für Schmerzen in der Mundschleimhaut ist oft ein Brennen, Stechen oderWundgefühl. Diverse mukosale Läsionen wie Ulzera, Erosionen und Vesikel sind häufige Ursachen von Schmerzen in der Mundschleimhaut.

Die Begriffe Stomatitis und orale Mukositis werden oft synonym verwendet, geben aber nicht ein- und dieselben Prozesse wieder. Stomatitis meint jeglichen Entzündungszustand der Mundschleimhaut, der aufgrund lokaler Infektionen oder Verletzungen oder systemischer Grunderkrankungen auftritt. Eine Mukositis tritt infolge von Bestrahlung oder Anwendung von Chemotherapeutika auf.

Eine ganze Palette lokaler mukosaler und systemischer Erkrankungen tritt aufgrund einer Entstehung von Ulzera oder Erosionen in Begleitung von Schmerzen auf. Diese Läsionen unterscheiden sich im Hinblick auf ihre Ausdehnung bis in die Mundschleimhaut.

- Ein mukosaler Ulkus ist definiert als Verlust von oberflächlichem Gewebe mit Zerfall und Nekrose von epithelialem Gewebe. Er geht mit einer Schädigung sowohl des Epithels wie auch der Lamina propria einher. Er durchdringt die Epithel-Bindegewebsschranke und seine Basis ist auf einer tiefen Schicht in der submukösen Bindegewebsschicht, in einigen Fällen sogar im Innern des Muskels oder der äußeren Knochenhaut.

- Eine mukosale Erosion ist definiert als oberflächliche Läsion in der Schleimhautmembran mit Schwund der oberflächlichen Epithelzellen und geringfügiger Schädigung der darunterliegenden Lamina propria.

\subsubsection{Schmerzen in der Mundschleimhaut zurückzuführen auf eine Mundschleimhautentzündung \\ Diagnostische Kriterien:}

A. Schmerzen, die die Kriterien für 1.2.1 Schmerzen in der Mundschleimhaut sowie Kriterium C erfüllen

B. Es wurde eine Entzündung der Mundschleimhaut diagnostiziert

C. Der Schmerz hat sich in einem engen zeitlichen Zusammenhang mit der Entzündung entwickelt oder führte zu ihrer Entdeckung

D. Nicht besser erklärt durch eine andere ICOP-Diagnose

Kommentar: Schmerzen in der Mundschleimhaut in Verbindung mit Geschwüren oder sonstigen Läsionen gehen oft mit hochgradigen schmerzbedingten unangenehmen Empfindungen einher. Die brennenden Schmerzen sind oft stark, und es kommt häufig zu einer funktionellen Beeinträchtigung des Mundraums (Essen, Sprechen) sowie von Lebensqualität und Schlaf.

1.2.1.1.1 Schmerzen in der Mundschleimhaut zurückzuführen auf ein Trauma oder eine Verletzung

\section{Diagnostische Kriterien:}

A. Schmerzen, die die Kriterien für 1.2.1.1 Schmerzen in der Mundschleimhaut zurückzuführen auf eine Mundschleimhautentzündung sowie Kriterium C erfüllen

B. Es ist ein Trauma oder eine Verletzung ${ }^{1}$ aufgetreten, wovon Mundschleimhautgewebe betroffen ist

C. Beide der folgenden Punkte sind erfüllt:

1. Der Schmerz ist im traumatisierten oder verletzten Gewebe lokalisiert
2. Der Schmerz hat sich innerhalb von Minuten bis Tagen nach einem Trauma oder einer Verletzung entwickelt

D. Nicht besser erklärt durch eine andere ICOP-Diagnose

\section{Anmerkung:}

1. Ein Trauma oder eine Verletzung kann unfallbedingt oder nicht unfallbedingt sein, von anderen zugefügt oder selbstzugefügt oder auch iatrogen, und wird teilweise bei den Unterformen spezifiziert.

1.2.1.1.1.1 Schmerzen in der Mundschleimhaut zurückzuführen auf ein nichtiatrogenes Trauma oder eine nichtiatrogene Verletzung

\section{Diagnostische Kriterien:}

A. Schmerzen, die die Kriterien für 1.2.1.1.1 Schmerzen in der Mundschleimhaut zurückzuführen auf ein Trauma oder eine Verletzung erfüllen

B. Das Trauma oder die Verletzung ${ }^{1}$ ist nichtiatrogen

\section{Anmerkung:}

1. Das Trauma kann mechanischer, thermischer oder chemischer Natur sein, unfallbedingt oder nichtunfallbedingt, von anderen oder selbst zugefügt.

Kommentar: Die Fälle von 1.2.1.1.1.1 Schmerzen in der Mundschleimhaut zurückzuführen auf ein nichtiatrogenes Trauma oder eine nichtiatrogene Verletzung umfassen nicht nur unfallbedingte Frontzahnverletzungen, sondern auch durch Mikrotraumen verursachte, zum Beispiel beim Essen oder Trinken zu heißer Speisen oder Getränke, und Trauma infolge von Zähneputzen oder Gebrauch von Zahnseide oder anderen Interdental-Instrumenten. Eine Untersuchung kann den ursächlichen Faktor offenbaren, etwa eine mandibuläre, maxillare oder dentoalveoläre Fraktur, Zahnwurzelfraktur oder lediglich eine Verletzung des Weichteilgewebes. Ein schlecht sitzender Zahnersatz kann schmerzhafte UIzerationen verursachen. Auch ein Zahnhochstand oder Parafunktionen (Beißen oder Kauen auf harten Objekten wie Nägeln, Stiften etc. oder gewohnheitsmäßiges Lippen-, Zungen- oder Wangenbeißen) können ein lokales Mundschleimhauttrauma mit hieraus resultierender Entzündung und Schmerzen nach sich ziehen. Verätzungen können mit einem Missbrauch von Antiphlogistika zusammenhängen (z. B. Lutschen von Tabletten, die dafür gedacht sind, geschluckt zu werden) oder Essen oder Trinken von zu heißen/scharfen Speisen oder Getränken. Als seltene Ursache eines Mundschleimhauttraumas kommt zudem Selbstverletzung vor. Bei Patienten mit Dystonie oder oraler Neuropathie kann die Verletzung rezidivierend sein.

Eine traumatische Verletzung der Mundschleimhaut verursacht eine akute Entzündung und kann in unterschiedlichem Ausmaß schmerzhaft sein. Eine traumatische Ulzeration der Mundschleimhaut kann akut oder chronisch sein, wobei Letzteres diagnostisch aufgrund einer zugrunde liegenden Fibrose und des klinischen Erscheinungsbildes einer neoplastischen Induration eine größere Herausforderung ist. Eine gründliche klinische Anamnese macht Kliniker oft auf eine traumatische Ätiologie oder Verbrennungen durch heißes Essen oder chemische Substanzen aufmerksam. 1.2.1.1.1.1 
Schmerzen in der Mundschleimhaut zurückzuführen auf ein nichtiatrogenes Trauma oder eine nichtiatrogene Verletzung können leicht bis stark sein und verschlimmern sich durch mechanische Provokation der Mundschleimhaut. Es kann Spontanschmerz auftreten.

1.2.1.1.1.2 Schmerzen in der Mundschleimhaut zurückzuführen auf eine chirurgische oder sonstige lokale iatrogene Verletzung

Diagnostische Kriterien:

A. Schmerzen, die die Kriterien für 1.2.1.1.1 Schmerzen in der Mundschleimhaut zurückzuführen auf ein Trauma oder eine Verletzung erfüllen

B. Das Trauma oder die Verletzung ist chirurgisch oder durch ein anderes lokales iatrogenes Verfahren ${ }^{1}$ verursacht

\section{Anmerkung:}

1. Mechanischer, thermischer oder chemischer Natur.

Kommentar: Zu den Ursachen von 1.2.1.1.1.2 Schmerzen in der Mundschleimhaut zurückzuführen auf eine chirurgische oder sonstige lokale iatrogene Verletzung gehören chirurgische Traumen und Verletzungen in Verbindung mit Zahn- und anderen oralen Behandlungen, etwa Verletzungen durch zahnärztliche Injektion sowie Verletzungen infolge direkter lokaler Komplikationen bei Eingriffen in der Mundhöhle. latrogene Verletzungen der Mundschleimhaut treten bei den meisten zahnchirurgischen Maßnahmen wie etwa Zahnextraktionen sowie bei gingival- oder parodontalchirurgischen Eingriffen auf. Thermische Verletzungen kommen beim Einsatz der Elektrokauterisation und des chirurgischen Lasers vor und chemische Verletzungen können nach unangemessenem Einsatz zum Beispiel von Desinfektionsmitteln oder Dentalwerkstoffen auftreten.

1.2.1.1.1.3 Schmerzen in der Mundschleimhaut zurückzuführen auf eine Bestrahlung oder Chemotherapie

\section{Diagnostische Kriterien:}

A. Schmerzen, die die Kriterien für 1.2.1.1.1 Schmerzen in der Mundschleimhaut zurückzuführen auf ein Trauma oder eine Verletzung erfüllen

B. Das Trauma oder die Verletzung ist auf eine Bestrahlung oder Chemotherapie zurückzuführen

Kommentar: Orale Mukositis ist ein Begriff, der erythematösen und ulzerativen Läsionen der Mundschleimhaut vorbehalten ist, wie sie bei Patienten vorkommen, die Strahlentherapie gegen Krebs im Kopf- und Halsbereich einschließlich der Mundhöhle erhalten oder Chemotherapie. Häufigkeit und Schweregrad variieren stark je nach Art und Dosis derTherapie. Die Läsionen manifestieren sich üblicherweise als sehr schmerzhafte Erytheme oder Ulzerationen, die die Nahrungsaufnahme und Mundhygiene beeinträchtigen sowie die Risiken einer lokalen und systemischen Infektion erhöhen. Der Zustand kann auch in Begleitung von Geschmacksstörungen und Mundtrockenheit auftreten.

Die Pathogenese der oralen Mukositis ist multifaktoriell; hinsichtlich ihrer Entstehung wurde ein komplexes Fünfstufenmodell vorgeschlagen.
Ohne Komplikationen durch eine Infektion heilt die Mukositis innerhalb von 2-4Wochen nach Einstellung der zytotoxischen Chemotherapie ab.

Die Mukositis kann sich durch lokale Faktoren und Infektionen verschlimmern. Auch wenn orale Komplikationen primär mit Beschwerden und einer Störung der oralen Funktion sowie einer Beeinträchtigung der Lebensqualität einhergehen, können diese Komplikationen bei Patienten, die auch immun- oder anderweitig geschwächt sind, lebensbedrohlich werden. Insbesondere können Infektionen in Verbindung mit Läsionen einer oralen Mukositis in Phasen einer weitreichenden Immunsuppression eine lebensbedrohliche systemische Sepsis auslösen. Daher ist das Management des Mukositisschmerzes eine primäre Komponente einer jeden Mukositis-Managementstrategie.

1.2.1.1.2 Schmerzen in der Mundschleimhaut zurückzuführen auf eine Infektion

\section{Diagnostische Kriterien:}

A. Schmerzen, die die Kriterien für 1.2.1.1 Schmerzen in der Mundschleimhaut zurückzuführen auf eine Mundschleimhautentzündung sowie Kriterium C erfüllen

B. Es wurde eine Infektion ${ }^{1}$ der Mundschleimhaut diagnostiziert ${ }^{2}$

C. Der Schmerz hat sich in einem engen zeitlichen Zusammenhang mit der Infektion entwickelt oder führte zu ihrer Entdeckung

D. Nicht besser erklärt durch eine andere ICOP-Diagnose

\section{Anmerkungen:}

1. Die Infektion kann durch Bakterien, Viren oder Pilze ausgelöst sein und wird unter der jeweiligen Unterform spezifiziert.

2. Die Diagnose stützt sich auf anamnestische Informationen, klinische Beobachtungen und/oder mikrobiologische Analysen.

Kommentar: Eine Infektion des Mundschleimhautgewebes verursacht eine akute Entzündung. 1.2.1.1.2 Schmerzen in der Mundschleimhaut zurückzuführen auf eine Infektion können leicht bis stark sein und verschlimmern sich durch mechanische Provokation der Mundschleimhaut. Es kann zum Auftritt von Spontanschmerz kommen.

Das Krankheitsbild wird entsprechend des ursächlichen Mikroorganismus den entsprechenden Unterkategorien zugeordnet.

1.2.1.1.2.1 Schmerzen in der Mundschleimhaut zurückzuführen auf eine bakterielle Infektion

Diagnostische Kriterien:

A. Schmerzen, die die Kriterien für 1.2.1.1.2 Schmerzen in der Mundschleimhaut zurückzuführen auf eine Infektion erfüllen

B. Es handelt sich um eine bakterielle Infektion

Kommentar: Bakterielle Infektionen sind die häufigsten oralen Infektionen. Eine bakterielle Infektion des Mundschleimhautgewebes verursacht eine akute Entzündung. Schmerzen in der Mundschleimhaut stehen oft in Verbindung mit einer zugrunde liegenden pathologischen Veränderung im Zahnbereich, mit einer parodontalen Infektion oder dentalen periapikalen Infektionen, die 
sich als Schwellung, Entzündung und Schmerzen der darüber liegenden Mundschleimhaut manifestieren.

1.2.1.1.2.1 Schmerzen in der Mundschleimhaut zurückzuführen auf eine bakterielle Infektion können leicht bis stark sein und verschlimmern sich durch mechanische Provokation der Mundschleimhaut. Es kann Spontanschmerz auftreten.

Eine akute nekrotisierende ulzerative Gingivitis (ANUG) oder nekrotisierende ulzerative Gingivitis (NUG), Parodontitis (NUP) oder Stomatitis (NUS) ist eine opportunistische Mundschleimhautinfektion, die durch diverse Bakterien bei unterernährten Kindern, jungen Erwachsenen und Immundefizitpatienten verursacht wird. NUG ist oft das anfängliche Erscheinungsbild und geht dann in NUP, NUS und letzten Endes Noma (eine Form von Gangrän, die das Gesicht befällt) über. Nekrose und Ulzeration der Mundschleimhaut, bohrender Schmerz, starker Mundgeruch, regionale Lymphadenopathie, Krankheitsgefühl und Fieber unterscheiden diese Form der Ulzeration von anderen. Kommt es zu einem Freiliegen des Alveolarknochens, kann sich nekrotischer Knochensequester entwickeln und sollte mit dem betroffenen Zahn zusammen entfernt werden.

Syphilis, verursacht durch eine Infektion mit Treponema pallidum, ist weiterhin recht verbreitet, mit steigenden Zahlen bei Männern mit gleichgeschlechtlichen Sexualkontakten. Die primäre Läsion zeigt sich am ersten Situs mukosaler Inokulation, oft der Mundschleimhaut. Ein hochgradig infektiöser, schmerzfreier solitärer Ulkus mit indurierten Rändern und ipsilateraler Lymphadenopathie tritt am häufigsten auf. Die Heilung erfolgt meist innerhalb von 3 Wochen. Nichtcharakteristische Schleimhautflecken sind ein Warnsignal für die Entwicklung einer sekundären Syphilis, häufig in Begleitung eines makulopulösen Ausschlags der palmoplantaren Hand- und Fußflächen und generalisierter Lymphadenopathie.

Gonorrhoische Läsionen können im Mund an einer Inokulationsstelle entstehen oder sekundär durch hämatogene Ausbreitung von einem primären Fokus anderswo. Die frühesten Symptome sind ein Brennen oder Jucken, Trockenheit oder Hitze im Mund, gefolgt von akuten Schmerzen beim Essen oder Sprechen. Am häufigsten sind die Mandeln und der Oropharynx beteiligt, und das Mundgewebe kann diffus entzündet oder ulzeriert sein. Der Speichel entwickelt eine erhöhte Viskosität und einen üblen Geruch. In ernsten Fällen kommt es zu einer submaxillären Lymphadenopathie mit Fieber.

Das Aufkommen des multiresistenten Mycobacterium tuberculosis (TB) und der hohen Zahlen von HIV-Infizierten in Ost- und Südafrika hat zu einer Zunahme der TB-Fälle geführt, was dringend eine Aufnahme in die Differenzialdiagnosen der orofazialen Pathologie nahelegt. Typisch ist eine sekundäre Tuberkulose in Form schmerzhafter, tiefer, unregelmäßiger Geschwüre mit induriertem Erscheinungsbild, unterminierten Rändern und dickem, schleimartigem Material an der Basis jedes Aspekts der Zunge. Eine hämatogene Ausbreitung von pulmonarer TB oder sekundäre Inokulation eines traumatischen Ulkus mit infiziertem Sputum ist die häufigste $\mathrm{Pa}$ thogenese. Eine primäre orale TB kommt eindeutig selten vor, üblicherweise in Verbindung mit dem Mycobacterium bovis. Die Ulzera ähneln einer chronischen traumabedingten Ulzeration und sogar einem Malignom, was dringend nach einer diagnostischen Biopsie verlangt. Häufig treten Begleitsymptome wie Schmerzen, Fieber, Lymphadenopathie, Heiserkeit und Gewichtsverlust mit den Ulzerationen auf.
Eine erworbene oder angeborene Immunsuppression kann zu einem erhöhten Risiko einer mukosalen Infektion führen. Patienten mit Immunsuppressionstherapie können eine Reihe opportunistischer Infektionen entwickeln, einschließlich einer pseudomembranösen Candidiasis und anderer Pilz- und Virusinfektionen. Eine Tumornekrosefaktor(TNF)-a-Therapie erhöht das TB-Risiko. Patienten mit Infliximab und Adalimumab mit kombinierter immunmodulatorischer Therapie können einem erhöhten Risiko von TB-, Histoplasmose- und Kokzidiomykose-Infektionen ausgesetzt sein. Antirheumatika wie Methotrexat, Abatacept und Alefacept zeigen ein erhöhtes Risiko von Herpes-simplex- und Herpes-Zoster-Infektionen und TB.

1.2.1.1.2.2 Schmerzen in der Mundschleimhaut zurückzuführen auf eine Virusinfektion

\section{Diagnostische Kriterien:}

A. Schmerzen, die die Kriterien für 1.2.1.1.2 Schmerzen in der Mundschleimhaut zurückzuführen auf eine Infektion erfüllen

B. Es handelt sich um eine virale Infektion'

\section{Anmerkung:}

1. Die Diagnose stützt sich auf eine mukosale Eruption im Schmerzareal zusammen mit der Bestimmung des Virus mittels Polymerase-Kettenreaktion (PCR) aus einem Abstrich des Areals.

Kommentar: Eine Virusinfektion des Mundschleimhautgewebes verursacht eine akute Entzündung. 1.2.1.1.2.2 Schmerzen in der Mundschleimhaut zurückzuführen auf eine Virusinfektion können leicht bis stark sein und sich durch mechanische Provokation der Mundschleimhaut verschlimmern. Es kann Spontanschmerz auftreten.

Zu den Virusinfektionen der Mundschleimhaut gehören Infektionen mit HSV, VZV, HPV, CMV, Coxsackieviren sowie die HIV-Infektion. Man beachte, dass es in der ICHD-3 einen spezifischen Kriterienkatalog für die Diagnose eines Herpes-Zoster-Virus gibt (4.1.2.1 Schmerzhafte Trigeminusneuropathie zurückzuführen auf Herpes Zoster).

Das infizierte Mundschleimhautgewebe kann oft ulzeriert und berührungsschmerzhaft sein. Oft fallen starke lokalisierte Schmerzen beim Essen oder Trinken bei heißen oder kalten Speisen oder Getränken auf. Der Schmerz wird beim Essen ausgelöst und kann so gravierend sein, dass die betroffene Person nicht in der Lage ist zu essen oder zu trinken, was zur Dehydratation führen kann.

Das Herpes-simplex-Virus (HSV) ist das häufigste Virus, das die Mundschleimhaut befällt. Eine herpetische Gingivostomatitis, die primäre HSV-1-Infektion, befällt meist Kinder und manifestiert sich entweder als asymptomatische Infektion oder mit mukosalen Vesikeln gefolgt von sich rasant entwickelnden schmerzhaften Ulzerationen, die sowohl die keratinisierte wie auch die nichtkeratinisierte Mundschleimhaut und das Zahnfleisch befallen. Oft sind die Schmerzen von Fieber, Unwohlsein, fauligem Geruch und zervikaler Lymphadenopathie begleitet. Erwachsene mit einer primären Infektion erleiden eine symptomatische herpetische Pharyngotonsillitis, eingangs als Bläschen, die schnell in flache Ulzerationen zerfallen. 
Rezidivierende Manifestationen des Virus in Form von Lippenherpes werden am häufigsten durch diverse Faktoren ausgelöst, darunter - aber ohne Beschränkung hierauf - Stress, UV-Exposition oder dentale Lokalanästhesie. Einem anfänglichen prodromalen Stechen oder Brennen folgt das Platzen einer Ansammlung von etwa fünf kleinen, flüssigkeitsgefüllten Bläschen auf erythematöser Schleimhaut, das schmerzhafte flache Geschwüre hinterlässt, die zusammenwachsen und verkrusten.

Herpangina (Hand-Mund-Fuß-Erkrankung), verursacht durch Coxsackieviren, ECHO-Virus und andere Enteroviren, befällt in der Regel Kinder unter 10 Jahren. Rötlichen Hautflecken oder Bläschen folgen selbstbegrenzende Ulzerationen von zirka $5 \mathrm{~mm}$ Durchmesser an den anterioren Tonsillensäulen, dem weichen Gaumen, dem Zäpfchen und/oder den Mandeln. Es kommt häufig zu Fieber, Halsschmerzen und Kopfschmerzen. Die Geschwüre heilen innerhalb von 4-6 Tagen ab.

Herpes Zoster (Gürtelrose) bedeutet die Reaktivierung einer schlummernden Infektion mit dem Varicella-Zoster-Virus (VZV oder HHV-3), die meist alte und geschwächte Patienten betrifft. Die Infektion ist hinreichend bekannt für den mit ihr verbundenen juckenden Hautausschlag mit Bläschenbildung, Ulzeration und Verkrustung, wobei dies alles gleichzeitig auftritt und dem Dermatom des Ganglions folgt, in dem das Virus Latenz hergestellt hat. Eine Verkrustung fehlt in der Mundschleimhaut, wo Läsionen sich stattdessen als ulzerierende Papeln manifestieren. Einem starken brennenden oder stechenden Schmerz im betroffenen Dermatom folgen mit Flüssigkeit gefüllte Bläschen, die aufplatzen und schmerzende flache Ulzerationen hinterlassen, die sich zu großflächigen denudierten Hautarealen zusammenfügen können. Orale Manifestationen signalisieren eine Beteiligung der mandibulären oder maxillären Äste des N. trigeminus, mit pathognomonischem abrupten Aufhören der Läsionen entlang der Mittellinie. Es wurde von einer Osteonekrose mit Zahnausfall berichtet, vor allem bei Menschen mit geschwächtem Immunsystem. Bei der Infektion sind oft mehrere Stellen im anatomischen Verteilungsgebiet des betroffenen Nervenasts betroffen (siehe auch 4.1.2.1 Schmerzhafte Trigeminusneuropathie zurückzuführen auf Herpes Zoster und 4.1.2.2 Postherpetische Trigeminusneuralgie).

Das humane Papillomvirus (HPV) kann einfache oder multiple papilläre Läsionen verursachen. Diese Läsionen sind selten schmerzhaft, sofern keine Traumatisierung erfolgt.

Das Epstein-Barr-Virus verursacht eine Mononukleose, welche mit Halsschmerzen und zahlreichen kleinen Ulzera einhergehen kann, die im Vorfeld einer Lymphadenopathie auftreten. Zu den sonstigen klinischen Merkmalen gehören Zahnfleischbluten und Petechien im Übergang zwischen dem weichen und dem harten Gaumen.

1.2.1.1.2.3 Schmerzen in der Mundschleimhaut zurückzuführen auf eine Pilzinfektion

\section{Diagnostische Kriterien:}
A. Schmerzen, die die Kriterien für 1.2.1.1.2 Schmerzen in der
Mundschleimhaut zurückzuführen auf eine Infektion erfüllen
B. Es handelt sich um eine Pilzinfektion

Kommentar: Eine Pilzinfektion des Mundschleimhautgewebes löst eine akute Entzündung aus. 1.2.1.1.2.3 Schmerzen in der Mundschleimhaut zurückzuführen auf eine Pilzinfektion können leicht bis stark sein und verschlimmern sich durch mechanische Provokation der Mundschleimhaut. Es kann zu Spontanschmerz kommen.

In jüngster Zeit hat die Prävalenz anderer oraler Pilzinfektionen als Candidiasis zugenommen. Immunschwächeerkrankungen wie eine HIV-Infektion und AIDS, eine immunsuppressive Therapie und längere Anwendung von Breitbandantibiotika und Kortikosteroiden gehören zu den hervorstechenden Gründen für ein Auftreten der Erkrankung, die bei einer gestörten Homöostase im Mundraum auftritt. Zu den sonstigen prädisponierenden Faktoren gehören Diabetes und Speicheldrüsenüberfunktion.

Die häufigste orale Pilzinfektion ist Candida albicans. Eine erythematöse Candidiasis äußert sich in Form von generalisierten Erythemen und Schmerzen. Die Glossitis rhombica mediana befällt die Zunge und weist drei Haupttypen auf: den pseudomembranösen Typ, der sich als weiße Flecken manifestiert, die sich leicht abwischen lassen und eine wunde, gerötete und blutende Oberfläche hinterlassen; den erythematösen Typ mit rötlichen Makulaläsionen und oft einem Brennen; und den Typ Cheilitis angularis, für den aufgesprungene Risse an den Mundwinkeln charakteristisch sind. Begleitsymptome sind Mundtrockenheit, Brennen, Stechen und Jucken sowie ein metallischer Geschmack.

Zu den sonstigen Mykosen, die es im Kontext von Schmerzen in der Mundschleimhaut in Betracht zu ziehen gilt, gehören Mukormykose, Aspergillose, Histoplasmose, Blastomykose und Parakokzidioidomykose. Zwar sind alle vergleichsweise selten, doch kommen am häufigsten Aspergillus- und Mucorales-Infektionen vor. Sie folgen auf die Einatmung der Sporen aus Erdreich, Mist, Getreide, Getreideprodukten oder verschimmeltem Mehl. Beide sind oberflächliche und invasive opportunistische Pilzinfektionen, die vor allem in der Mundhöhle immungeschwächter Patienten anzutreffen sind.

1.2.1.1.3 Schmerzen in der Mundschleimhaut zurückzuführen auf eine Autoimmunreaktion

\section{Diagnostische Kriterien:}

A. Schmerzen, die die Kriterien für 1.2.1.1 Schmerzen in der Mundschleimhaut zurückzuführen auf eine Mundschleimhautentzündung sowie Kriterium C erfüllen

B. Es wurde eine Autoimmunerkrankung oder -störung diagnostiziert, die bekanntermaßen Schmerzen in der Mundschleimhaut ${ }^{1}$ auslösen kann

C. Der Schmerz hat sich in einem engen zeitlichen Zusammenhang mit der Autoimmunerkrankung oder -störung entwickelt oder führte zu ihrer Entdeckung

D. Nicht besser erklärt durch eine andere ICOP-Diagnose

\section{Anmerkung:}

1. Hierzu gehören Pemphigus, Schleimhautpemphigoid, rezidivierende aphthöse Stomatitis, oraler Lichen planus, Erythema multiforme, Sjögren-Syndrom, Morbus Behçet, Graft-versusHost-Reaktion, Lupus erythematodes (systemischer oder diskoider Typus), Erythema migrans, Morbus Crohn, Colitis ulcerosa und Zöliakie. 
Kommentar: 1.2.1.1.3 Schmerzen in der Mundschleimhaut zurückzuführen auf eine Autoimmunreaktion können leicht bis stark sein und verschlimmern sich durch mechanische Provokation der Mundschleimhaut. Es können sowohl evozierte als auch spontane Schmerzen auftreten. Die Prognose für den Schmerz hängt vom Ergebnis der Behandlung der zugrundeliegenden Autoimmunerkrankung ab.

Mehrere dermatologische immunvermittelte vesiculo-ulzerative Krankheitsbilder können eine Beteiligung der Mundschleimhaut zeigen, sei es gleichzeitig mit der pathologischen Veränderung der Haut, als anfängliches klinisches Bild oder mitunter als das einzige klinische Bild.

Pemphigus, eine Gruppe immunvermittelter subepithelialer bullöser Dermatosen, werden durch Autoantikörper vermittelt, die sich gegen die Adhäsionsproteine der Keratinozyten (Desmosomen) richten und eine Akantholyse bewirken. Pemphigus vulgaris betrifft am häufigsten die Mundhöhle, wobei sich die Autoantikörper in erster Linie gegen Desmoglein-1 und 3 (mukokutane Formen) oder nur 3 (mukosale Formen) richten. Gingivale Schmerzen aufgrund von Pemphigus sind selten, da die Erkrankung meist die Mundschleimhaut befällt. Die Patienten, üblicherweise 40-60 Jahre alt, weisen schlaffe intraepitheliale Blasen mit dünnem Dach auf, die unmittelbar nach ihrer Entstehung aufplatzen, was zu großfächigen, unregelmäßigen Arealen schmerzhafter mukosaler Ulzerationen führt.

Das Schleimhautpemphigoid (engl. Mucous membrane pemphigoid, MMP) ist eine häufige systemische blasenbildende $\mathrm{Au}$ toimmunerkrankung vorzugsweise mit Beteiligung der Schleimhäute. Die Antikörper richten sich gegen die Keratinozyten-Proteine für die Adhäsion der Bindegewebsmatrix oder Hemi-Desmosomen (BP180 und Laminin-332), was bewirkt, dass das Epithel sich von seinem daruntergelegenen Bindegewebsbett löst. Der subepitheliale Charakter der Ablösung resultiert in Bläschen mit dickem Dach, die bei der Untersuchung noch intakt sein können. Eine Ruptur der Vesikel hinterlässt ulzerative Läsionen ohne Epithel, bedeckt von gelblich-weißem Schorf.

Die rezidivierende Stomatis aphthosa (engl. Recurrent aphthous stomatitis, RAS) stellt die häufigste Form von Ulzeration der Mundschleimhaut bei ansonsten gesunden Personen dar. Der Begriff sollte rezidivierenden Ulzera der Mundschleimhaut vorbehalten sein, die mit keinerlei systemischen Erkrankungen in Verbindung stehen und üblicherweise in der Kindheit oder Jugend beginnen. Am häufigsten betroffen ist die nichtkeratinisierte Schleimhaut der Mundhöhle, der Lippen und des weichen Gaumens. Eine Reihe lokaler und systemischer Faktoren, darunter immunologische, allergische, ernährungsbezogene, mikrobielle Organismen und psychosozialer Stress, ebenso wie Immunsuppressiva, wurden als mögliche ätiologische Faktoren ins Spiel gebracht. Eine erhöhte Prävalenz bei engen Angehörigen verweist auch auf einen möglichen genetischen Hintergrund. RAS zeigt bei HIV-infizierten Patienten ein atypisches klinisches Bild und sollte bei solchen Patienten immer in die Differenzialdiagnose der Mundschleimhautulzerationen einbezogen werden. Wenn RAS erst in späteren Lebensjahren beginnt, können zusätzliche mukosale Oberflächen betroffen sein, und eine umfangreiche medizinische Anamnese und körperliche Untersuchung sollten erwogen werden, um eine entzündliche gastrointestinale
Erkrankung wie Morbus Crohn, Zöliakie, Morbus Behçet, SweetSyndrom, zyklische Neutropenie, HIV-Infektion und Arzneimittelreaktionen auszuschließen, die sich alle in aphthösen Geschwüren manifestieren können. Klinisch betrachtet wird RAS in Unterkategorien untergliedert: Die Minor-Form mit kleineren Aphthen, die häufigste Variante, die sich üblicherweise mit ein bis fünf Ulzera von weniger als $10 \mathrm{~mm}$ Durchmesser und umgeben von einem flammend roten Entzündungshof zeigt, die innerhalb von 10-14 Tagen spontan abheilen, und die Major-Form (Morbus Sutton), die sich als tiefere, großflächigere (gewöhnlich $>10 \mathrm{~mm}$ Durchmesser), persistierende Ulzerationen mit unregelmäßigen Grenzen manifestieren und in der Regel Wochen oder Monate brauchen, bis sie abheilen.

Der orale Lichen planus (OLP) ist eine relative häufige chronische Entzündungsreaktion, die hauptsächlich Frauen mittleren Alters betrifft. Seine Pathogenese bleibt ungewiss, doch spielen diverse Untergruppen von T-Lymphozyten und Mastzellen eine Rolle bei der hier anzutreffenden Schädigung der Basalmembran. Das Spektrum der klinischen Erscheinungsbilder dieser Erkrankung variiert und umfasst eine atrophische, eine erosive, eine ulzerative und - weniger häufig - eine bullöse Variante. Die Läsionen betreffen üblicherweise die beidseitige Mundschleimhaut und sind recht symmetrisch. Sie zeigen sich entweder einzig als Erkrankung der Mundschleimhaut oder treten in Begleitung einer desquamativen Gingivitis und/oder kutaner Manifestationen auf. Im Falle des erosiven und des ulzerativen Typs beobachtet man schmerzhafte, von einer Pseudomembran bedeckte Ulzerationen umrandet von blassweißen Streifungen (Striae) in einer multifokalen Verteilung. Aktuelle Metaanalysen ermittelten für OLP eine maligne Transformationsrate von insgesamt etwa $1 \%$, wobei am häufigsten die Zunge älterer Frauen betroffen ist; doch dieser Punkt bleibt umstritten.

Erythema multiforme (EM) ist eine T-Zellen-vermittelte zytotoxische Immunreaktion vom Typ IV auf eine Reihe von Antigenen (viraler, bakterieller, pharmakologischer oder chemischer Art), die zu einem apoptosevermittelten Epithelzelltod führt. Kürzlich wurden Anti-Desmoplakin-I- und -II-Antikörper als möglicher maßgeblicher Faktor bei der zytotoxischen Reaktion nachgewiesen. EM betrifft meist junge, ansonsten gesunde Personen und kehrt oft zeitgleich mit rezidivierenden HSV-Infektionen wieder. Orale Läsionen können entweder den Beginn einer weiteren mukokutanen Beteiligung kennzeichnen oder treten isoliert auf, klassischerweise mit geschwollenen, rissigen, hämorrhagischen und verkrusteten Lippen mit oder ohne Blasenbildung auf der Mundschleimhaut und Ulzerationen.

Das Sjögren-Syndrom ist eine systemische Autoimmunerkrankung, die oft gleichzeitig mit anderen systemischen Bindegewebs- oder organspezifischen Autoimmunerkrankungen auftritt. Die Verbindung ist gut beschrieben für systemischen Lupus erythematodes und rheumatoide Arthritis. Das Zahnfleischgewebe kann von trockenen Nahrungsmitteln abgescheuert und sogar eingeschnitten werden, und es kann wund werden. Das Vorliegen des Sjögren-Syndroms beeinflusst in einem bestimmten Umfang die Expression der anderen Autoimmunerkrankungen, zum Beispiel durch Erhöhung der Müdigkeit und des Lymphomrisikos.

Morbus Behçet ist eine multisystemische Autoimmunerkrankung unbekannter Ätiologie. Kennzeichnend für das Krankheitsbild sind Mundgeschwüre, Genitalgeschwüre und Augenentzündung. 
Es kann neben einer neurologischen und vaskulären Beteiligung zu dermatologischen Symptomen kommen. Die Mundläsionen sind schmerzhaft und manifestieren sich zyklisch; sie befallen Lippen, Wangenschleimhaut, weichen Gaumen und Zunge und ähneln vom Erscheinungsbild aphthösen Läsionen, mit einem Durchmesser im Bereich von wenigen Millimetern bis Zentimetern. Es besteht eine vermehrte Inzidenz der Erkrankung in der Bevölkerung des Mittelmeerraums und Asiens, vor allem in der Türkei.

Die Graft-versus-Host-Reaktion ist von lichenoiden, papulösen und erythematösen Läsionen gekennzeichnet sowie gelegentlich Ulzerationen und Desquamation an der Wangen- und Lippenschleimhaut, am Gaumen und dem rückwärtigen Teil der Zunge. Die oralen Läsionen sind oft von Fieber, Krankheitsgefühl, Übelkeit und Mundtrockenheit begleitet. Die Befunde im Mundraum können durch eine Kombination von Radiotherapie, Chemotherapie, Immunsuppressiva und sekundären Infektionen verursacht sein.

Mehr als die Hälfte der Patienten mit systemischem Lupus erythematodes (SLE) können orale Läsionen aufweisen, am häufigsten Ulzeration und Schmerzen in der Wangenschleimhaut und den Lippen während der frühen, aktiven Krankheitsphase. Ulzerative Läsionen und erythematöse Läsionen mit oder ohne ausstrahlende weiße Striae können auch als Bestandteil des klinischen Spektrums eines diskoiden Lupus erythematodes (DLE) in Erscheinung treten. DLE gilt als potenziell maligne Erkrankung der Mundschleimhaut aufgrund der erhöhten Prävalenz oraler Plattenepithelzellkarzinome innerhalb dieser Population, vor allem bezogen auf die Unterlippe.

Erythema migrans (Lingua geographica, gutartige migratorische Glossitis) ist eine häufige Entzündung des Mundraums unbekannter Ätiologie, mit einer geschätzten Prävalenz von 1-3\%. Rund $30 \%$ der Patienten berichten von Beschwerden im Mundraum, einer brennenden und stechenden Empfindung. In der Regel ist die Zunge betroffen, obwohl auch andere Stellen im Mundraum betroffen sein können. Zum Erscheinungsbild können kreisförmige gerötete Areale gehören, oft durch erhabene, weißliche Grenzbereiche scharf umrissen und an den lateralen, dorsalen, anterioren und/oder ventralen Teilen der Zunge lokalisiert. Das erythematöse Erscheinungsbild geht auf Atrophie und Schwund der Papillae filiformes zurück. Am häufigsten wird eine Verbindung zu Atopie und Psoriasis postuliert. Die Erkrankung sollte nicht mit dem charakteristischen Ausschlag als Frühmanifestation einer Lyme-Borreliose verwechselt werden.

Morbus Crohn manifestiert sich mit multifokalen, linearen, nodulären oder diffusen Schleimhautverdickungen in der Lippen- und Wangenschleimhaut und den mukobukkalen Falten. Sie kann mit schmerzhaften, anhaltenden aphthösen Ulzerationen und einer atrophischen Glossitis einhergehen.

Die Colitis ulcerosa manifestiert sich mit verstreuten, zusammengeklumpten oder linear ausgerichteten Pusteln auf einer erythematös veränderten Schleimhaut an verschiedenen Stellen des Mundbereichs. Einige Patienten weisen zusätzlich zu den pustulösen Läsionen schmerzhafte orale aphthöse Läsionen auf. Zöliakie kann sich mit Schmerzen in den Schleimhäuten manifestieren, in der Regel in Verbindung mit aphthösen Ulzera. Eine Eisen- und Vitamin-B-Resorptionsstörung kann zu brennenden, stechenden Empfindungen in der Zunge führen.
Zu den sonstigen seltenen autoimmunbezogenen oder idiopathischen Ursachen von Schmerzen und einer Empfindlichkeit verursachenden Mundschleimhautulzeration gehören ein eosinophiles Ulkus, Riesenzellarteriitis, Hypereosinophilie-Syndrom, nekrotisierende Sialometaplasie, Polyarteriitis nodosa, reaktive Arthritis (Reiter-Syndrom), akute febrile neutrophile Dermatose (Sweet-Syndrom) und Wegenersche Granulomatose.

1.2.1.1.4 Schmerzen in der Mundschleimhaut zurückzuführen auf eine Überempfindlichkeit oder allergische Reaktion

\section{Diagnostische Kriterien:}

A. Schmerzen, die die Kriterien für 1.2.1.1 Schmerzen in der Mundschleimhaut zurückzuführen auf eine Mundschleimhautentzündung sowie Kriterium C erfüllen

B. Es ist eine Überempfindlichkeit oder allergische Reaktion in der Mundschleimhaut ${ }^{1}$ aufgetreten

C. Der Schmerz hat sich in einem engen zeitlichen Zusammenhang mit der Überempfindlichkeit oder allergischen Reaktion entwickelt

D. Nicht besser erklärt durch eine andere ICOP-Diagnose

\section{Anmerkung:}

1. Die Überempfindlichkeits- oder allergische Reaktion kann in Verbindung mit Dentalwerkstoffen (wie etwa von Provisorien oder auch bleibenden Restaurationen oder Abdruckmaterial) auftreten, einem Mundhygieneprodukt, einem topischen Medikament, einem systemischen Arzneimittel, Lebensmittel oder Lebensmittelzusatz oder einem anderen Faktor.

Kommentar: 1.2.1.1.4 Schmerzen in der Mundschleimhaut zurückzuführen aufeine Überempfindlichkeit oder allergische Reaktion können leicht bis stark sein und sie verschlimmern sich durch mechanische Provokation der Mundschleimhäute. Es kann zum Auftritt sowohl von evoziertem wie auch Spontanschmerz kommen.

Das orale Allergiesyndrom (OAS) tritt üblicherweise bei Individuen auf, die allergisch auf Pollen von Bäumen, Gräsern oder Getreide reagieren. Frischobst, Rohkost und rohe Nüsse sind häufige Ursachen von OAS. Zu den Symptomen können ein Jucken und/ oder eine Schwellung der Gesamtheit oder eines Teils der Lippen, der Zunge, des Mundes und/oder des Rachens gehören, aber diese können gelegentlich schwer sein und sogar mit Übelkeit und Erbrechen einhergehen.

Dentalwerkstoffe, Mundhygieneprodukte und Lebensmittelzusätze können als Reaktion Kontaktallergien im Mund mit verschiedenen klinischen Erscheinungsbildern verursachen, darunter Stomatitis, lichenoide Läsionen, Erosionen, Blasen und Ulzerationen.

Allergische und Überempfindlichkeitsreaktionen der Mundschleimhaut sind weniger häufig als kutane, vermutlich aufgrund einer Allergenverdünnung und eines ständigen Reinigungseffekts des normalen Speichelflusses. Läsionen können sich mit unspezifischen Gewebsödemen, Erythemen, Rissigkeit, Ulzeration, hyperkeratotischen weißen Plaques und/oder mukosaler Desquamation präsentieren.

Von einer allergischen Kontaktstomatitis, wenngleich eine solche selten auftritt, wurde in Verbindung mit Zahnabdruckwerkstoffen, Zahnrestaurationswerkstoffen, der Anwendung von topischem 
Benzocain und - noch häufiger - Zimt in Zahncremes, Mundspülungen und Kaugummi berichtet. Läsionen, lokalisiert oder weit gestreut, können als gemischte rote und weiße Flecken mit Ulzeration, Schwellung der Wangen und Desquamation zutage treten, die sich auf den Lippen, den Wangen, der Zunge und dem Zahnfleisch zeigen.

Eine Überempfindlichkeitsreaktion auf ein systemisches Medikament oder eine Substanz, mit der ein direkter Kontakt besteht und welche die entsprechende Reaktion auslöst, kann zu klinischen und histologischen Merkmalen führen, die an einen Lichen ruber planus erinnern. Es werden die Begriffe orale lichenoide Arzneimittelreaktion (engl. Oral lichenoid drug reaction, OLDR) beziehungsweise orale lichenoide Kontaktläsion (engl. Oral lichenoid contact lesion, $\mathrm{OLCL}$ ) verwendet, und beide können sich mit erheblicher Ulzeration manifestieren, üblicherweise mit Erythemen und weißlichen Streifungen an der Peripherie der Ulzeration. Oft ist bei einem OLCL Amalgam beteiligt, bestätigt durch einen Patch-Test, der eine Überempfindlichkeit gegen Quecksilber oder Amalgam attestiert. OLDR begegnet man mit einer gewissen Häufigkeit bei mit Angiotensin-konvertierendes-Enzym-Hemmern (ACE-Hemmern), nichtsteroidalen Entzündungshemmern (NSAP) und oralen Antidiabetika behandelten Patienten.

Im Falle einer arzneimittelassoziierten Überempfindlichkeit können Läsionen lange nach Beginn der Medikamenteneinnahme beginnen und noch Monate nach Einstellung der Einnahme bestehen bleiben, was Diagnose und Management erschwert.

Ein fixes Arzneimittelexanthem (engl. Fixed drug eruption, FDE) ist eine Form von Überempfindlichkeit, die aufgrund ihrer Gebundenheit an eine bestimmte anatomische Stelle auffällig ist und in Verbindung mit NSAP und anderen Oxicamen, Gabapentin, Fuconazol und systemischen antibakteriellen und antimykotischen Medikamenten auftritt. Ein fixes Arzneimittelexanthem sollte in solchen Fällen in Betracht gezogen werden, in denen ein zeitlicher Zusammenhang mit der Einnahme des Medikaments besteht. Der Nachweis kann durch Patch-Test oder orale Provokationstests erbracht werden; das Management erfolgt durch Meiden des Medikaments oder Ersatz durch ein anderes, während die akuten Läsionen mit topischen oder systemischen Steroiden behandelt werden können.

Eine arzneimittelinduzierte fibroepitheliale Hyperplasie oder fibrovaskuläre Hyperplasie kann gelegentlich mit einem schmerzhaften Erscheinungsbild in Verbindung stehen, wahrscheinlich aufgrund einer zugrunde liegenden parodontalen Infektion, verursacht durch Schwierigkeiten mit der Mundhygiene bei diesen Erkrankungen.

1.2.1.1.5 Schmerzen in der Mundschleimhaut zurückzuführen auf eine Entzündung der Mundschleimhaut anderen Ursprungs

\section{Diagnostische Kriterien:}

A. Schmerzen, die die Kriterien für 1.2.1.1 Schmerzen in der Mundschleimhaut zurückzuführen auf eine Mundschleimhautentzündung sowie Kriterium C erfüllen

B. Es wurde eine Störung diagnostiziert, die bekanntermaßen eine Entzündung der Mundschleimhaut auslösen kann, jedoch anderer Art ist als unter 1.2.1.1.1 bis 1.2.1.1.41 dargelegt

C. Die Schmerzursache ist klinisch plausibel

D. Nicht besser erklärt durch eine andere ICOP-Diagnose

\section{Anmerkung:}

1. Zu derartigen Störungen gehören endokrine Störungen oder Veränderungen, Ernährungsdefizite, hämatologische Erkrankungen, gastrointestinale und dermatologische Erkrankungen sowie arzneimittelinduzierte Störungen (nicht auf eine Überempfindlichkeit oder Allergie zurückzuführen).

Kommentar: 1.2.1.1.5 Schmerzen in der Mundschleimhaut zurückzuführen auf eine Entzündung der Mundschleimhaut anderen Ursprungs können leicht bis stark sein und verschlimmern sich durch mechanische Provokation der Mundschleimhaut. Es kann Spontanschmerz auftreten.

Eine Veränderung des physiologischen Zustands wie etwa in der Schwangerschaft und in den Wechseljahren können zu endokrinen Veränderungen führen, die sich als Beschwerden und Schmerzen in der Mundschleimhaut manifestieren. Zu den systemischen Erkrankungen, die dort eine Entzündung der Mundschleimhaut und Schmerzen verursachen können, gehören endokrine Erkrankungen (Schilddrüsenunterfunktion, Diabetes mellitus), Ernährungsdefizite (Eisen-, Vitamin-B-Komplex-, Zinkmangel), Magen-Darm-Störungen und medikamenteninduzierte Störungen (nicht zurückzuführen auf eine Überempfindlichkeit oder Allergie).

Ein Eisen-, Vitamin-B12- und Folsäuremangel kann eine atrophische Glossitis auslösen, bei der die Papillae filiformes des Zungenrückens atrophieren und eine glatte, erythematöse Zunge zurücklassen. Auch andere Teile der Mundschleimhaut können atrophisch und gerötet wirken. Aphthöse Ulzera sind bei schweren Fällen gang und gäbe. Brennende oder stechende Empfindungen können klinisch nachweisbaren oralen Läsionen vorausgehen. Schwere Fälle von Vitamin-B12-Mangel können auch von Parästhesie begleitet sein. Patienten können eine Neigung zur Entwicklung von Mundwinkelrhagaden haben. Hämatologische Erkrankungen wie Anämie, Gammopathien, hämatinische Mängel, Leukämie, myelodysplastisches Syndrom, Neutropenie und andere Leukozytendyskrasien können zu einer spröden Mundschleimhaut mit hieraus resultierender Ulzeration und Schmerzen führen.

Störungen des Magen-Darm-Trakts wie eine gastroösophageale Refluxstörung und ein Magengeschwür können zu einer Resorptionsstörung und hiermit zusammenhängenden Mangelernährung führen und in der Folge zu entsprechenden Schmerzen in der Mundschleimhaut.

Zu den dermatologischen Ursachen schmerzhafter mukosaler Läsionen gehören Dermatitis herpetiformis, lineare IgA-Dermatose, Epidermolysis bullosa und chronisch-ulzerative Stomatitis. Bei einer durch antineoplastische Therapie induzierten Mukositis ist eine komplexe Kaskade von Ereignissen involviert, die durch reaktive Sauerstoffspezies initiiert werden, mit ausgedehnter Entzündung, Atrophie, Schwellung, Hautrötung und Geschwürbildung. Hierzu zählen eine durch eine Chemotherapie induzierte Mukositis ebenso wie Strahlenläsionen. Letztere treten an den exponierten Oberflächen auf, während chemotherapieinduzierte Mukositis den gesamten Verdauungstrakt betrifft. Typ und Dosierung systemischer zytotoxischer Wirkstoffe sowie die Dosierung und das Strahlenfeld haben Auswirkung auf das Vorhandensein und den Schweregrad der Mukositis. Evidenzgestützte Leitlinien für das Management einer durch Krebstherapie induzierten Mundschleimhautentzün- 
dung sind etabliert, und auf diese sollte bei allen Fällen von Patienten Bezug genommen werden, die diese Mittel erhalten. Gutartige hyperplastische Läsionen oder Tumoren mit Beteiligung der Mundschleimhaut sind üblicherweise nicht unmittelbar mit Schmerzen verbunden, können jedoch schmerzen, wenn sie durch Okklusionsstörungen oder Zahnersatz traumatisiert und/oder infiziert werden (siehe 1.2.1.1.1 Schmerzen in der Mundschleimhaut zurückzuführen auf ein Trauma oder eine Verletzung und 1.2.1.1.2 Schmerzen in der Mundschleimhaut zurückzuführen auf eine Infektion).

\subsubsection{Schmerzen in der Mundschleimhaut zurückzuführen auf eine maligne Läsion}

\section{Diagnostische Kriterien:}

A. Schmerzen, die die Kriterien für 1.2.1 Schmerzen in der Mundschleimhaut sowie Kriterium C erfüllen

B. Es wurde eine Neoplasie der Mundschleimhaut diagnostiziert

C. Ein ursächlicher Zusammenhang ist aufgrund der anatomischen und/oder zeitlichen Verbindung plausibel

D. Nicht besser erklärt durch eine andere ICOP-Diagnose

Kommentar: 1.2.1.2 Schmerzen in der Mundschleimhaut zurückzuführen auf eine maligne Läsion können leicht bis stark sein und verschlimmern sich durch mechanische Provokation der Mundschleimhaut. Es kann zum Auftreten von Spontanschmerz kommen.

Die Mundschleimhaut kann von einer ganzen Reihe primärer wie auch metastasierender Malignome betroffen sein, die alle das Bild unspezifischer Geschwüre zeigen können. Orale Plattenepithelkarzinome sind am häufigsten und zeigen sich häufig als Ulzeration mit klinischer Induration, Fixierung am darunterliegenden Gewebe, eingerollten exophytischen Rändern sowie Schmerzen und/oder Taubheit.

\subsubsection{Schmerzen der Speicheldrüse}

Beschreibung: Schmerzen verursacht durch eine Läsion oder Störung, von der die Speicheldrüsen betroffen sind.

\section{Diagnostische Kriterien:}

A. Schmerzen im Speicheldrüsengewebe, die Kriterium C erfüllen

B. Klinische Befunde, Laborwerte, bildgebende Verfahren und/ oder die Anamnese belegen eine Läsion oder Störung der Speicheldrüsen, die bekanntermaßen Schmerz auslösen kann ${ }^{1}$

C. Ein ursächlicher Zusammenhang lässt sich durch beide der folgenden Kriterien nachweisen:

1. Der Schmerzort entspricht der/den Lokalisation(en) der Läsion oder Störung

2. Eines oder beides von Folgendem trifft zu:

a) Der Schmerz entstand in einem zeitlichen Zusammenhang mit dem Auftreten oder Beginn der Läsion oder Störung

b) Der Schmerz verschlimmert sich bei Ausübung von Druck auf die betroffene Speicheldrüse

D. Nicht besser erklärt durch eine andere ICOP-Diagnose

\section{Anmerkung:}

1. Die Läsion oder Störung wird bei der jeweiligen Unterform spezifiziert.

1.2.2.1 Schmerzen der Speicheldrüse zurückzuführen auf eine obstruktive Ursache

\section{Diagnostische Kriterien:}

A. Schmerzen, die die Kriterien für 1.2.2 Schmerzen der Speicheldrüse sowie Kriterium C erfüllen

B. Es wurde eine Obstruktion des Speichelgangs ${ }^{1}$ diagnostiziert

C. Der Schmerz hat sich in einem engen zeitlichen Zusammenhang mit der Obstruktion entwickelt oder führte zu ihrer Entdeckung

D. Nicht besser erklärt durch eine andere ICOP-Diagnose

\section{Anmerkung:}

1. Die Obstruktion kann auf eine Sialolithiasis zurückgehen, einen Schleimpfropfen, eine raumfordernde Läsion oder eine traumatische beziehungsweise iatrogene Verletzung der Speicheldrüse oder des Speichelgangs.

Kommentar: Patienten mit einer Obstruktion des Speichelgangs zeigen meistens eine Vorgeschichte akuter intermittierender Schmerzen und Schwellung der betroffenen großen Speicheldrüse.

Der Grad des Schmerzes und der Schwellung hängt von Umfang der Obstruktion des Speichelgangs und dem Vorliegen einer Sekundärinfektion ab.

Zu den selteneren Ursachen für Schmerzen im Bereich der Speicheldrüse gehören gut- und bösartige Speicheldrüsentumoren. Diese gehen in der Regel nicht unmittelbar mit Schmerzen einher, können jedoch Schmerzen auslösen, die mit der Obstruktion der Drüse oder des Gangs zusammenhängen.

Unter den iatrogenen Ursachen sind therapiebedingte Verletzungen, zum Beispiel durch Jod-131: Die Speicheldrüsenfunktion ist nach Ablationstherapie mit hochaktivem Radiojod bei Patienten mit gut differenziertem Schilddrüsenkarzinom betroffen. Radioaktives Jod wird im Speicheldrüsengewebe aktiv akkumuliert, und eine Sialadenitis ist eine gängige Folgeerkrankung, zusammen mit einer verminderten Speichelsekretion und Mundtrockenheit, die zu einer Infektion der Speicheldrüse und Schmerzen führt.

\subsubsection{Schmerzen der Speicheldrüse zurückzuführen auf eine Infek-} tion

\section{Diagnostische Kriterien:}

A. Schmerzen, die die Kriterien für 1.2.2 Schmerzen der Speicheldrüse sowie Kriterium C erfüllen

B. Es wurde eine Infektion ${ }^{1}$ der Speicheldrüse(n) diagnostiziert ${ }^{2}$

C. Der Schmerz hat sich in einem engen zeitlichen Zusammenhang mit der Infektion entwickelt oder führte zu ihrer Entdeckung

D. Nicht besser erklärt durch eine andere ICOP-Diagnose

\section{Anmerkungen:}

1. Die Infektion kann bakterieller oder viraler Art sein und wird bei der jeweiligen Unterform spezifiziert. 
2. Die Diagnose stützt sich auf anamnestische Informationen, klinische Beobachtungen und/oder die mikrobiologische Analyse.

1.2.2.2.1 Schmerzen der Speicheldrüse zurückzuführen auf eine bakterielle Infektion

\section{Diagnostische Kriterien:}

A. Schmerzen, die die Kriterien für 1.2.2.2 Schmerzen der Speicheldrüse zurückzuführen auf eine Infektion erfüllen

B. Es handelt sich um eine bakterielle Infektion

Kommentar: Die häufigste bakterielle Ursache ist eine Staphylokokken-Infektion.

Eine bakterielle Sialadenitis kann akut oder chronisch sein. Primärer prädisponierender Faktor ist ein verminderter Speichelfluss, was eine retrograde mikrobielle Besiedelung des Speichelgangs erlaubt und zur Entstehung einer akuten oder chronischen eitrigen Infektion führen kann. Kennzeichnend für eine akute Sialadenitis ist eine schmerzhafte Schwellung einer einzelnen Speicheldrüse, wobei im Regelfall die Ohrspeicheldrüse betroffen ist. Ein eitriger Ausfluss kann aus der Öffnung des Speichelgangs kommen, und der Patient kann eine Rötung der darüberliegenden Haut oder sogar eine Abszessbildung im Gewebe der entzündeten Drüse, Unwohlsein, Fieber und eine zervikale Lymphadenopathie aufweisen. Eine bakterielle Sialadenitis tritt oft bei immungeschwächten $\mathrm{Pa}$ tienten und älteren Patienten auf, die aufgrund systemischer Erkrankungen, durch Medikamenteneinnahme oder Dehydratation unter einer Unterfunktion der Speicheldrüse leiden, oder sie kann mit einer Obstruktion der Speichelgänge durch Ablagerung von Speichelsteinen, Schleimpfropfen, einem Tumorwachstum oder Trauma in Verbindung stehen. Eine chronische Sialadenitis kann sich im Gefolge einer akuten Sialadenitis entwickeln, falls sich die prädisponierenden Faktoren nicht ausschalten lassen.

1.2.2.2.2 Schmerzen der Speicheldrüse zurückzuführen auf eine Virusinfektion

\section{Diagnostische Kriterien:}

A. Schmerzen, die die Kriterien für 1.2.2.2 Schmerzen der Speicheldrüse zurückzuführen auf eine Infektion erfüllen

B. Es handelt sich um eine Virusinfektion

Kommentar: Zu den Virusinfektionen der Speicheldrüsen gehören Mumps, HIV- und CMV-Infektion, die zusätzlich zur Schwellung Schmerzen verursachen können.

Mumps zeigt meist Auswirkungen auf die Ohrspeicheldrüse, mit beidseitiger plötzlicherVergrößerung, Berührungsempfindlichkeit, doch bei bis zu $25 \%$ der Fälle findet sich eine einseitige Schwellung. Starker lokaler Schmerz wird oft in Verbindung mit Kieferbewegungen beim Sprechen und Kauen festgestellt, vor allem wenn es zu einer partiellen Obstruktion des Speichelgangs kommt. Im typischen Fall sind Kinder im Alter von 4-6 Jahren betroffen.
1.2.2.3 Schmerzen der Speicheldrüse zurückzuführen auf eine chronische rezidivierende Parotitis des Kindesalters

\section{Diagnostische Kriterien:}

A. Schmerzen, die die Kriterien für 1.2.2 Schmerzen der Speicheldrüse sowie Kriterium C erfüllen

B. Es wurde eine chronisch rezidivierende Parotitis des Kindesalters diagnostiziert

C. Der Schmerz ist in einem engen zeitlichen Zusammenhang mit dem Beginn oder Wiederauftritt der Parotitis entstanden bzw. rezidiviert

D. Nicht besser erklärt durch eine andere ICOP-Diagnose

Kommentar: Die juvenile rezidivierende Parotitis ist eine häufige Erkrankung der Speicheldrüsen im Kindesalter, charakterisiert durch eine intermittierende Schwellung der Ohrspeicheldrüse auf einer Seite oder beidseits, mit oder ohne Schmerzen. Sie geht im Allgemeinen mit einer nichtobstruktiven Sialektasie der Ohrspeicheldrüse sowie Speicheldrüsenunterfunktion einher. Es zeigt sich eine biphasische Altersverteilung, mit Spitzen im Alter von 2-5 und 10 Jahren. Die häufigsten Symptome sind Schwellungen, Schmerzen und Fieber. Die Symptome beschränken sich auf eine Dauer von etwa 3 Tagen und können des Öfteren auftreten, mit etwa acht Episoden im Jahr.

Die Diagnosestellung erfolgt basierend auf der medizinischen Vorgeschichte und einer Bestätigung per Sialographie oder Ultraschall. Die Ätiologie ist unklar, doch bei den meisten Patienten legt sich die chronische rezidivierende Parotitis des Kindesalters im Erwachsenenalter.

1.2.2.4 Schmerzen der Speicheldrüse zurückzuführen auf eine immunologisch vermittelte Störung

\section{Diagnostische Kriterien:}

A. Schmerzen, die die Kriterien für 1.2.2 Schmerzen der Speicheldrüse sowie Kriterium C erfüllen

B. Es wurde eine immunologisch vermittelte Störung diagnostiziert, die bekanntermaßen Schmerzen im Bereich der Speicheldrüse ${ }^{1}$ auslösen kann

C. Der Schmerz hat sich in einem engen zeitlichen Zusammenhang mit der Störung entwickelt oder führte zu ihrer Entdeckung

D. Nicht besser erklärt durch eine andere ICOP-Diagnose

\section{Anmerkung:}

1. Die wichtigste von diesen ist das Sjögren-Syndrom.

Kommentar: Das Sjögren-Syndrom ist eine Autoimmunerkrankung, die zu einer Funktionsstörung der Speicheldrüsen führt. Zu den Symptomen zählen eine rezidivierende oder persistierende Schwellung der Speicheldrüsen, Mundtrockenheit, Schwierigkeiten mit dem Kauen, Schmerzen und Brennen der Mundschleimhaut, chronische Halsschmerzen und Schluckbeschwerden. 
1.2.2.5 Schmerzen der Speicheldrüse zurückzuführen auf eine andere Ursache

\section{Diagnostische Kriterien:}

A. Schmerzen, die die Kriterien für 1.2.2 Schmerzen der Speicheldrüse sowie Kriterium C erfüllen

B. Es wurde eine Läsion oder Störung diagnostiziert, die bekanntermaßen Schmerzen im Bereich der Speicheldrüse auslösen kann, allerdings anderer Art als die in 1.2.2.1 bis 1.2.2.4 ${ }^{1}$ dargelegten

C. Der Schmerz hat sich in einem engen zeitlichen Zusammenhang mit der Läsion oder Erkrankung entwickelt oder führte zu ihrer Entdeckung

D. Nicht besser erklärt durch eine andere ICOP-Diagnose

\section{Anmerkung:}

1. Eine andere Ursache von Schmerzen im Bereich der Speicheldrüse kann eine allogene Transplantation mit Graft-versusHost-Reaktion (GVHD) sein. Die Speicheldrüsen gehören zu den durch GVHD am meisten befallenen Organen. Die Erkrankung manifestiert sich in Form von Hyposalivation und Mundtrockenheit, Infektion und in der Folge Schmerzen.

\subsubsection{Schmerzen im Kieferknochen}

Beschreibung: Schmerzen verursacht durch eine Läsion oder Störung, an der das Kieferknochengewebe beteiligt ist.

\section{Diagnostische Kriterien:}

A. Schmerzen im Kiefer', die Kriterium C erfüllen

B. Es wurde klinisch, laborchemisch, mittels Bildgebung und/ oder anamnestisch eine Läsion oder Störung des Kieferknochens $^{2}$ nachgewiesen, die bekanntermaßen Schmerz auslösen kann

C. Ein ursächlicher Zusammenhang lässt sich durch beide der folgenden Kriterien nachweisen:

1. Der Schmerz ist am Situs der Kieferknochenläsion lokalisiert $^{1}$

2. Eines oder beides von Folgendem trifft zu:

a) Der Schmerz hat sich in zeitlichem Zusammenhang mit dem Auftreten oder Beginn der Kieferknochenläsion oder -störung entwickelt

b) Der Schmerz verschlimmert sich durch Ausübung von Druck auf die Kieferknochenläsion

D. Nicht besser erklärt durch eine andere ICOP-Diagnose

\section{Anmerkungen:}

1. Der Schmerz kann auch zu anderen Stellen des ipsilateral-orofazialen Gebiets übertragen werden und/oder dorthin ausstrahlen.

2. Die Läsion oder Störung wird bei der jeweiligen Unterform spezifiziert.

1.2.3.1 Schmerzen im Kieferknochen zurückzuführen auf ein Trauma oder eine Verletzung

\section{Diagnostische Kriterien:}

A. Schmerzen, die die Kriterien für 1.2.3 Schmerzen im Kieferknochen sowie Kriterium C erfüllen
B. Es ist ein Trauma oder eine Verletzung mit Beteiligung des Kieferknochens eingetreten

C. Der Schmerz hat sich in einem engen zeitlichen Zusammenhang mit einem Trauma oder einer Verletzung entwickelt

D. Nicht besser erklärt durch eine andere ICOP-Diagnose

Kommentar: Zu den Kieferknochenverletzungen zählt etwa eine Kieferfraktur. Sportarten wie Fußball, Baseball und Hockey sowie Zusammenstöße im Rahmen von Verkehrsunfällen machen unter jungen Erwachsenen einen hohen Prozentsatz von Gesichtsverletzungen aus. Insbesondere treten Unterkieferfrakturen in Begleitung von Platzwunden am Kinn auf.

Eine Unterkieferfraktur kann vorliegen, wenn der Patient nur begrenzt in der Lage ist, den Mund zu öffnen, oder wenn es zu einer anormalen Mundöffnung kommt; auch eine Okklusionsstörung legt nahe, dass eine Unterkieferfraktur vorliegt. Das Gleiche gilt für ein Taubheitsgefühl im Kinn unmittelbar nach dem Trauma.

\subsubsection{Schmerzen im Kieferknochen zurückzuführen auf eine Infek-} tion

\section{Diagnostische Kriterien:}

A. Schmerzen, die die Kriterien für 1.2.3 Schmerzen im Kieferknochen sowie Kriterium C erfüllen

B. Es wurde eine Infektion' des Kieferknochengewebes diagnostiziert

C. Der Schmerz hat sich in einem engen zeitlichen Zusammenhang mit der Infektion entwickelt oder führte zu ihrer Entdeckung

D. Nicht besser erklärt durch eine andere ICOP-Diagnose

\section{Anmerkung:}

1. Die Infektion kann durch Bakterien, Viren oder Pilze verursacht sein und wird bei jeder Unterform spezifiziert.

Kommentar: Intraossäre bakterielle, virale und Pilzinfektionen können Schmerzen im Kieferknochen verursachen. Überwiegend handelt es sich um bakterielle Infektionen.

Die Infektion kann als Folge einer Osteo(radio)nekrose des Oberund Unterkiefers auftreten, was zu den Schmerzen in Verbindung mit einer Osteonekrose beitragen kann (siehe 1.2.3.5 Schmerzen im Kieferknochen zurückzuführen auf eine Therapie).

1.2.3.2.1 Schmerzen im Kieferknochen zurückzuführen auf eine bakterielle Infektion

Diagnostische Kriterien:

A. Schmerzen, die die Kriterien für 1.2.3.2 Schmerzen im Kieferknochen zurückzuführen auf eine Infektion erfüllen

B. Es handelt sich um eine bakterielle Infektion

Kommentar: Zu den bakteriellen Infektionen des Kieferknochengewebes gehört die Osteomyelitis. Odontogene Infektionen können sich ausbreiten und eine Osteomyelitis des Kiefers verursachen; eine Osteomyelitis als Folge einer odontogenen Infektion ist jedoch relativ selten. Starke Kieferschmerzen sind ein gängiges Symptom einer Kieferosteomyelitis und können in Begleitung von Anästhesie 
oder Hypästhesie auf der betroffenen Seite auftreten. Bei langwierigen Fällen kann sich eine Kieferklemme entwickeln.

\subsection{Schmerzen im Kieferknochen zurückzuführen auf eine} Virusinfektion

\section{Diagnostische Kriterien:}

A. Schmerzen, die die Kriterien für 1.2.3.2 Schmerzen im Kieferknochen zurückzuführen auf eine Infektion erfüllen

B. Es handelt sich um eine virale Infektion

Kommentar: Zu den Virusinfektionen des Kieferknochengewebes gehören Herpes Zoster (HZ, Gürtelrose), resultierend aus der Reaktivierung des Varicella-Zoster-Virus. Zu den Komplikationen zählt eine HZ-induzierte Osteonekrose. Ungewöhnliche Zahnkomplikationen wie Knochennekrose, Zahnausfall, Parodontitis, verkalkte Wurzelkanäle und devitalisierte Pulpa, periapikale Läsionen und Wurzelresorption und Entwicklungsstörungen wie etwa unregelmäßige verkürzte Zahnwurzeln und fehlende Zähne können als Folge der Beteiligung des zweiten oder dritten Trigeminusabschnitts durch $\mathrm{HZ}$ entstehen.

\subsection{Schmerzen im Kieferknochen zurückzuführen auf eine} Pilzinfektion

\section{Diagnostische Kriterien:}

A. Schmerzen, die die Kriterien für 1.2.3.2 Schmerzen im Kieferknochen zurückzuführen auf eine Infektion erfüllen

B. Es handelt sich um eine Pilzinfektion

Kommentar: Die wahrscheinlichsten Pilzinfektionen des Kieferknochengewebes sind Aspergillose und Mukormykose.

Eine Aspergillose der Mundhöhle ist eine seltene Erkrankung, die überwiegend bei immungeschwächten Patienten auftritt, etwa solchen mit hämatologischen Malignomen. Osteomyelitis verursacht durch Aspergillen ist eine häufig vernachlässigte Infektion. Eine invasive orale Aspergillose kommt zwar nur selten vor, stellt jedoch eine potenziell tödliche Erkrankung dar und sollte bei immunsupprimierten Patienten mit oralen Läsionen in Betracht gezogen werden.

Mukormykose ist eine seltene opportunistische Infektion, die meist immungeschwächte Patienten befällt, aber im Ausnahmefall auch ansonsten gesunde Personen nach einer Zahnextraktion. Der bei der Mukormykose beteiligte Organismus ist ein saprophytischer Pilz, in der Hauptsache Rhizopus oder Mucor. Es handelt sich um die tödlichste und die am rasantesten fortschreitende Form humaner Pilzinfektionen.

\subsubsection{Schmerzen im Kieferknochen zurückzuführen auf eine lokale benigne Läsion}

\section{Diagnostische Kriterien:}

A. Schmerzen, die die Kriterien für 1.2.3 Schmerzen im Kieferknochen sowie Kriterium C erfüllen

B. Es wurde eine lokale gutartige Läsion diagnostiziert, die bekanntermaßen Schmerzen im Bereich des Kieferknochengewebes auslösen kann'
C. Der Schmerz hat sich in einem engen zeitlichen Zusammenhang mit der Läsion entwickelt oder führte zu ihrer Entdeckung

D. Nicht besser erklärt durch eine andere ICOP-Diagnose

\section{Anmerkung:}

1. Zu den lokalen gutartigen Läsionen gehören Riesenzelltumor, Osteoidosteom und Osteoblastom.

Kommentar: Gutartige Knochentumoren sind oft asymptomatisch und werden bei der Begutachtung eines Traumas oder sonstigen Krankheitsbildes als Zufallsbefund entdeckt. Wenn sie symptomatisch sind, können gutartige Knochentumoren sich mit lokalisierten Schmerzen, Schwellungen, einer Fehlstellung oder pathologischen Fraktur manifestieren. Die meisten gutartigen Knochentumore weisen im Röntgenbild bestimmte charakteristische Merkmale auf. Erweiterte bildgebende Verfahren (z. B. Computertomographie [CT], Magnetresonanztomographie [MRT]) können erforderlich sein, um den Charakter von Knochentumoren vollständig zu ermitteln.

Der Riesenzelltumor des Knochens ist ein relativ seltenes, gutartiges osteolytisches Skelettneoplasma bei jungen Erwachsenen. Das häufigste Erscheinungsbild besteht in Schmerz und Schwellung. Schädel- und Gesichtsschädelknochen sind weniger häufig beteiligte Situs.

Patienten mit Osteoidosteom klagen üblicherweise über einen progressiv zunehmenden Schmerz, der bei Nacht schlimmer und unabhängig von aktiver Betätigung ist. Der Schmerz wird durch Aspirin oder andere NSAR gelindert, in der Regel innerhalb von 2025 Minuten. Eine ausbleibende Schmerzlinderung durch die Wirkstoffe sollte ein Signal dafür sein, andere Diagnosen in Betracht zu ziehen.

Patienten mit Osteoblastom klagen üblicherweise über chronische Dauerschmerzen. Die radiologischen Befunde bei einem Osteoblastom sind variabel und oft sind erweiterte bildgebende Verfahren (z. B. CT oder MRT) zur näheren Identifikation erforderlich. Der Schmerz lässt sich durch Aspirin oder andere NSAP nicht lindern.

1.2.3.4 Schmerzen im Kieferknochen zurückzuführen auf eine maligne Läsion

\section{Diagnostische Kriterien:}

A. Schmerzen, die die Kriterien für 1.2.3 Schmerzen im Kieferknochen sowie Kriterium C erfüllen

B. Es wurde eine primäre oder sekundäre bösartige Läsion diagnostiziert, die bekanntermaßen Schmerzen im Kieferknochengewebe ${ }^{1}$ auslösen kann

C. Der Schmerz hat sich in einem zeitlichen Zusammenhang mit der Läsion entwickelt oder zu ihrer Entdeckung geführt

D. Nicht besser erklärt durch eine andere ICOP-Diagnose

\section{Anmerkung:}

1. Schmerzen im Kieferknochen können auf die direkte Massenwirkung primärer oder metastasierender Tumoren oder auf die paraneoplastische Wirkung metastasierender Tumoren zurückgehen. 
Kommentar: Schmerzen im Kieferknochen zurückzuführen auf maligne Läsionen, ob primär oder metastatisch, können mit lokalisierten Schmerzen auftreten, die im Laufe von Wochen oder Monaten zu- und abnehmen können.

1.2.3.4.1 Schmerzen im Kieferknochen zurückzuführen auf einen lokalen Tumor

\section{Diagnostische Kriterien:}

A. Schmerzen, die die Kriterien für 1.2.3.4 Schmerzen im Kieferknochen zurückzuführen auf eine maligne Läsion erfüllen

B. Die maligne Läsion ist primärer $\mathrm{Art}^{1}$

\section{Anmerkung:}

1. Zu den primären malignen Läsionen, die bekanntermaßen Schmerzen im Kieferknochengewebe auslösen können, gehören Osteosarkom, Langerhans-Zell-Histiozytose, Non-Hodgkin-Lymphom und multiples Myelom.

Kommentar: Ein Osteosarkom ist zwar ein seltener Tumor, doch - abgesehen von Myelomen - bei weitem der wahrscheinlichste primäre bösartige Tumor, der im Knochen entsteht (obwohl oft als sekundär betrachtet, zurückzuführen auf eine sarkomatöse Transformation ausgehend von einer vorliegenden Paget-Krankheit des Knochens oder anderen gutartigen Knochenläsionen). Mehrheitlich zeigen Patienten mit Osteosarkom lokalisierte Schmerzen, üblicherweise über mehrere Monate. Der Schmerz beginnt häufig nach einer Verletzung und kann über ein paar Wochen oder Monate immer wieder zu- und abnehmen. Systemische Symptome wie Fieber, Gewichtsverlust und Krankheitsgefühl fehlen generell. Die häufigsten beteiligten Lokalisationen sind der distale Oberschenkelknochen, das proximale Schienbein und der proximale Oberschenkelknochen; ein Befall der Kieferknochen tritt eher selten auf.

Bei der Langerhans-Zell-Histiozytose (LCH) zeigen radiologische Untersuchungen üblicherweise ein lytisches, ,, ausgestanztes" Erscheinungsbild, mitunter in Verbindung mit einer begleitenden Weichteilmasse. Als Leitsymptome können Schmerzen im Kiefer und lockere Zähne auftreten. Zwar können Knochenläsionen in einigen Arealen asymptomatisch verlaufen, im Mund jedoch verursachen sie aufgrund von Zahnverlust und einer hohen Rezidivrate die größten Beschwerden. Die hinteren Regionen der Kieferknochen sind häufiger betroffen als die vorderen.

Das Non-Hodgkin-Lymphom ist ein Tumor des lymphatischen Systems, der entweder von B- oder von T-Lymphozyten ausgeht und ein hohes Malignitätspotenzial aufweist. Unspezifische Symptome wie etwa unklare primäre Zahnschmerzen und eine nicht zurückgehende periapikale Schwellung können die korrekte Diagnose eines Non-Hodgkin-Lymphoms erschweren, was häufig zu einer verspäteten Diagnose führt. Es empfiehlt sich ein Scan des Ober- und Unterkiefers per CT oder eine digitale Volumentomographie (DVT) sowie immunhistochemische Färbung der Biopsate. Befällt die Läsion die Kieferknochen, so gilt dies im Vergleich zum Oberkiefer nur selten für den Unterkiefer: Bei den berichteten Fällen betreffen nur 0,6\% den Oberkiefer.

Ein multiples Myelom ist eine Erkrankung, bei der Plasmazellen im Knochenmark zu wuchern beginnen, oft mit dem Resultat einer ausgedehnten Zerstörung des Skeletts mit osteolytischen Lä- sionen, Osteopenie und/oder pathologischen Frakturen. Knochenschmerzen, vor allem im Rücken oder der Brust und weniger häufig in den Extremitäten, liegen zum Zeitpunkt der Diagnose bei schätzungsweise $60 \%$ der Patienten vor.

1.2.3.4.2 Schmerzen im Kieferknochen zurückzuführen auf einen Ferntumor

\section{Diagnostische Kriterien:}

A. Schmerzen, die die Kriterien für 1.2.3.4 Schmerzen im Kieferknochen zurückzuführen auf eine maligne Läsion erfüllen

B. Der Tumor ist weiter entfernt lokalisiert

Kommentar: Maligne Läsionen anderenorts können durch direkte Massenwirkungen Schmerzen verursachen (darunter Nervenkompression und Periostdehnung) und einen paraneoplastischen Effekt (eine Fernwirkung ohne metastatische Ausbreitung in den/ die Kiefer) ausüben.

\subsubsection{Schmerzen im Kieferknochen zurückzuführen auf eine The-} rapie

\section{Diagnostische Kriterien:}

A. Schmerzen, die die Kriterien für 1.2.3 Schmerzen im Kieferknochen sowie Kriterium C erfüllen

B. Ein therapeutischer Eingriff hat zu einer Läsion oder Störung geführt, die bekanntermaßen Schmerzen im Kieferknochen ${ }^{1}$ auslösen kann

C. Die Schmerzursache ist basierend auf dem anatomischen $\mathrm{Zu}$ sammenhang mit der Läsion oder Störung und/oder der zeitlichen Verbindung ${ }^{2}$ mit seinem Auftreten oder Beginn plausibel

D. Nicht besser erklärt durch eine andere ICOP-Diagnose

\section{Anmerkungen:}

1. Zu Läsionen oder Störungen dieser Art gehören eine arzneimittelbedingte Osteonekrose des Ober- und Unterkiefers (engl. Medication-related osteonecrosis of the jaws, MRONJ), Osteoradionekrose und Alveolitis sicca (trockenes Zahnfach) nach Zahnziehung.

2. Der Schmerz entwickelt sich üblicherweise innerhalb von Stunden bis Tagen nach dem Auftreten der ursächlichen Läsion. Die Zeit, bis die Läsion in Erscheinung tritt, ist jedoch therapiespezifisch: Die Läsion kann nach einer Intervention wie etwa einem chirurgischen Eingriff unmittelbar auftreten, nach Einleitung einer Arzneimitteltherapie oder Bestrahlung jedoch gegebenenfalls Monate bis Jahre später.

Kommentar: Eine medikamentenassoziierte Osteonekrose der Kiefer (MRONJ) besteht definitionsgemäß bei Vorliegen von nekrotischem Knochen (der freiliegt oder sich mit der Diagnosesonde durch eine Nebenhöhle austasten lässt) für mehr als 8 Wochen im Kiefer- und Gesichtsbereich einer Person, die mit Bisphosphonat oder anderen antiresorptiven (z. B. Denosumab) oder antiangiogenen (z. B. Bevacizumab) Arzneimitteln behandelt wurde. MRONJ manifestiert sich üblicherweise als Schmerz, Infektion und nekrotischer Knochen im Ober- oder Unterkiefer von Patienten, die diese Mittel erhalten. Ein wesentlicher Risikofaktor sind dentoalveoläre Operationen. 
Osteoradionekrose ist eine Komplikation der Strahlentherapie (RT) aufgrund eines vaskulären Verschlusses und einer verminderten Gefäßversorgung des bestrahlten Gewebes. Zu den Symptomen einer Osteoradionekrose können Schmerzen, Mundgeruch, Störung des Geschmacksempfindens, Dysästhesie oder Anästhesie, Kieferklemme, Probleme mit dem Kauen und Schlucken, Schwierigkeiten mit dem Sprechen, Fistelbildung, pathologische Fraktur und Infektionen gehören. Die Zeit bis zum Einsetzen einer Osteoradionekrose variiert stark. In einigen Fällen kann diese kurz nach Abschluss der Strahlentherapie diagnostiziert werden, während sie bei anderen Patienten unter Umständen für Jahre nach der ursprünglichen Krebsbehandlung nicht diagnostiziert wird. Der am häufigsten befallene Knochen ist der Unterkiefer; eine maxilläre Osteoradionekrose hingegen kommt selten vor.

Die Alveolitis sicca (trockenes Zahnfach) ist eine Komplikation nach Zahnextraktion und tritt häufiger in Verbindung mit dem Ziehen von Unterkiefermolaren auf. Sie wird von heftigen Schmerzen begleitet, die sich 2-3 Tage postoperativ entwickeln. Typisch ist ein Zahnfach, das ganz oder teilweise frei von Blutgerinnseln ist. Bei einigen Patienten besteht Mundgeruch.

\subsubsection{Schmerzen im Kieferknochen zurückzuführen auf eine syste- mische Erkrankung}

\section{Diagnostische Kriterien:}

A. Schmerzen, die die Kriterien für 1.2.3 Schmerzen im Kieferknochen sowie Kriterium C erfüllen

B. Es wurde eine systemische Erkrankung diagnostiziert, die bekanntermaßen Schmerzen im Kieferknochen ${ }^{1}$ verursachen kann

C. Die Schmerzursache ist klinisch plausibel

D. Nicht besser erklärt durch eine andere ICOP-Diagnose

\section{Anmerkung:}

1. Zu diesen Erkrankungen gehören Sichelzellenanämie, Morbus Gaucher und Morbus Paget.

Kommentar: Einige systemische Erkrankungen manifestieren sich mit rezidivierenden vasookklusiven Schmerzepisoden, charakterisiert von diffusen Knochenschmerzen, unterbrochen von schmerzhaften Krisen, und führen oft zu einer Osteonekrose (Knochennekrose).

Charakteristisch für eine Sichelzellenanämie ist eine deutliche Heterogenität im Hinblick auf den klinischen und hämatologischen Schweregrad, mit wiederholten gefäßokklusiven Schmerzepisoden als Kennzeichen. Diese können durchaus jede Woche auftreten, oder es vergehen lange Zeitspannen ohne solche. Schmerzepisoden können zu Knocheninfarkten, Nekrose und im Laufe der Zeit degenerativen Veränderungen in Knochenmark enthaltenden Knochen führen und so, zusätzlich zu den akuteren schmerzhaften Episoden, zu einem chronischen Schmerzzustand.

Morbus Gaucher ist eine angeborene Stoffwechselstörung, die sich auf das Recycling zellularer Glykolipide auswirkt. Sie stellt eine der häufigsten lysosomalen Speicherkrankheiten dar. Kennzeichnend für die Skeletterkrankung sind diffuse Knochenschmerzen, mit Auftreten von schmerzhaften Krisen, die oft in eine Osteonekrose (Knochennekrose) münden.
Die Paget-Krankheit des Knochens (engl. Paget's disease of bone, PDB) ist auch unter dem historischen Namen Osteitis deformans bekannt. PDB ist eine fokale Störung des Knochenstoffwechsels, charakterisiert durch eine beschleunigte Knochenumbaurate, was zu Knochenüberwucherung an einem einzelnen Situs (monostotische PDB) oder an mehreren Stellen (polyostotische PDB) führt. Zu den häufig betroffenen Arealen gehören der Schädel, die Wirbelsäule, das Becken und die langen Knochen der unteren Extremität. Ähnlich wie beim Osteosarkom können diese mit lokalisierten Schmerzen und einer lokalisierten Schwellung auftreten und finden sich üblicherweise bei Patienten mit polyostotischer Erkrankung.

\section{Literatur}

\subsubsection{Schmerzen der Zahnpulpa}

Abbott PV und Yu C. A clinical classification of the status of the pulp and the root canal system. Austral Dent J 2007; 52(suppl): S. 17-31.

Benoliel R, Sharav Y und Eliav E. Neurovascular orofacial pain. J Am Dent Assoc 2010 141: 1094-1096. Berman LH und Hartwell GR. Diagnosis. In: Cohen S and Hargreaves KM (eds) Pathways of the pulp. 9. Ausg. St Louis, MO: Mosby-Elsevier, 2006, S.2-39.

Byers MR und Narhi MVO. Nerve supply of the pulpodentin complex and response to injury. In: Hargreaves KM und Goodis HE (Hg.) Seltzer and Bender's dental pulp. Chicago, IL: Quintessence, 2002, S. 151-179.

Falace DA, Reid K und Rayens MK. The influence of deep (odontogenic) pain intensity, quality, and duration on the incidence and characteristics of referred orofacial pain. J Orofac Pain 1996; 10: 232-239.

Garfunkel A, Sela J und Ulmansky M. Dental pulp pathosis: clinicopathologic correlations based on 109 cases. Oral Surg Oral Med Oral Pathol 1973; 35: 110-117.

Glick DH. Locating referred pulpal pains. Oral Surg Oral Med Oral Pathol 1962; 15: 613-623.

Hargreaves KM und Seltzer S. Pharmacologic control of dental pain. In: Hargreaves KM und Goodis HE (Hg.) Seltzer and Bender's dental pulp. Chicago, IL: Quintessence, 2002, pp.205-225.

Hasler JE und Mitchell DF. Painless pulpitis. J Am Dent Assoc 1970; 81: 671-677.

Kang, SH, Kim BS und Kim Y. Cracked teeth: distribution, characteristics, and survival after root canal treatment. J Endod 2016; 42: 557-562.

Mejare IA, Axelsson S, Davidson T, et al. Diagnosis of the condition of the dental pulp: a systematic review. Int Endod J 2012; 45: 597-613.

Michaelson PL und Holland GR. Is pulpitis painful? Int Endod J 2002; 35: 829-832.

Obermann M, Mueller D, Yoon MS, et al. Migraine with isolated facial pain: a diagnostic challenge. Cephalalgia 2007; 27: 1278-1282.

Patel S, Kanagasingam S und Pitt Ford T. External cervical resorption: a review. J Endod 2009; 35: 616-625.

Schuurmans TJ, Nixdorf DR, Idiyatullin DS, et al. Accuracy and reliability of root crack and fracture detection in teeth using magnetic resonance imaging. J Endodontics 2019; 45: 750-755.

Seo DG, Yi YA, Shin SJ, et al. Analysis of factors associated with cracked teeth J Endod 2012; 38: 288-292.

Sharav Y, Katsarava Z und Benoliel R. Migraine and possible facial variants: neurovascular orofacial pain. In: Sharav Y and Benoliel R (eds) Orofacial pain and headache. 2nd ed. Chicago, IL: Quintessence Publishing Co., 2015, S. 319-362.

Sharav $Y$, Katsarava Z und Charles A. Facial presentations of primary headache disorders. Cephalalgia 2017; 37: 714-719.

Sharav Y, Leviner E, Tzukert A, et al. The spatial distribution, intensity and unpleasantness of acute dental pain. Pain 1984; 20: 363-370.

Sigurdsson A. Clinical manifestations and diagnosis. In: Ørstavik D and Pitt-Ford TR (eds) Essential endodontology. 2nd ed. Oxford: Blackwell Munksgaard, 2008, S. 235-261.

Smulson MH und Sieraski SM. Histophysiology and diseases of the dental pulp In: Weine FS (ed.) Endodontic therapy. 5. Ausg. St Louis, MO: Mosby, 1996, S. 84-165.

Tyldesley WR und Mumford JM. Dental pain and the histological condition of the pulp. Dent Pract Dent Rec 1970; 20: 333-336. 
Von Troil B, Needleman I und Sanz M. A systematic review of the prevalence of root sensitivity following periodontal therapy. J Clin Periodontol 2002; 29(3 suppl): 173-177.

\subsubsection{Parodontale Schmerzen}

Andreasen JO and Andreasen FM. Classification, etiology and epidemiology of traumatic dental injuries. In: Andreasen JO (ed.) Textbook and color atlas of traumatic injuries to the teeth. 3rd ed. Copenhagen: Munksgaard, 1994, S. 151-177.

Armitage GC. Development of a classification system for periodontal diseases and conditions. Ann Periodontol 1999; 4: 1-6.

Bastone EB, Freer TJ und McNamara JR. Epidemiology of dental trauma: a review of the literature. Aust Dent J 2000; 45: 2-9.

Costa CPS, Thomaz EBAF und Souza S de FC. Association between sickle cell anemia and pulp necrosis. J Endod 2013; 39: 177-181.

Gomes BP, Lilley JD und Drucker DB. Associations of endodontic symptoms and signs with particular combinations of specific bacteria. Int End J 1996; 29: 69-75.

Gutmann JL, Baumgartner JC, Gluskin AH, et al. Identify and define all diagnostic terms for periapical/periradicular health and disease states. J Endod 2009; 35: 1658-1674.

Heasman PA und Hughes FJ. Drugs, medications and periodontal disease. Br Dent J 2014; 217: 411-418.

Horning GM und Cohen ME. Necrotizing ulcerative gingivitis, periodontiits, and stomatitis: clinical staging and predisposing factors. J Periodontol 1995; 66: 990-998.

Kaste LM, Gift HC, Bhat M, et al. Prevalence of incisor trauma in persons 6 to 50 years of age: United States 1988-1991. J Dent Res 1996; 75: 696-705 (Sonderausgabe).

Kinane JF und Chestnutt IG. Smoking and periodontal disease. Crit Rev Oral Biol Med 2000; 11: 356-365.

Laux M, Abbott PV, Pajarola G, et al. Apical inflammatory root resorption: a correlative radiographic and histological assessment. Int Endod J 2000; 33: 483-493.

Levin LG, Law AS, Holland GR, et al. Identify and define all diagnostic terms for pulpal health and disease states. J Endod 2009; 35: 1645-1657.

Mejàre IA, Axelsson S, Davidson T, et al. Diagnosis of the condition of the dental pulp: a systematic review. Int Endod J 2012; 45: 597-613.

Ricucci D und Siqueira JF Jr. Biofilms and apical periodontitis: study of prevalence and association with clinical and histopathologic findings. Endod 2010; 36: 1277-1288.

Ricucci D, Siqueira JF Jr, Lopes WS, et al. Extraradicular infection as the cause of persistent symptoms: a case series. J Endod 2015; 41: 265-273.

Sharav $Y$ und Benoliel R. Acute orofacial pain. In: Sharav $Y$ and Benoliel R (eds) Orofacial pain and headache. 2nd ed. Chicago, IL: Quintessence Publishing Co., 2015, pp.141-161.

Sjo“ gren U, Happonen RP, Kahnberg K-E, et al. Survival of Arachnia propionica in periapical tissue. Int Endodont J 1988; 21: 277-282.

The Dental Trauma Guide - ein online verfügbarer, evidencegestützter Behandlungsleitfaden, herausgegeben in Zusammenarbeit mit dem Resource Centre for Rare Oral Diseases und Department of Oral and Maxillofacial Surgery, Copenhagen University Hospital, www.dentaltraumaguide.org/(abgerufen Januar 2020).

Tronstad L, Barnett F, Riso K, et al. Extraradicular endodontic infections. Endod Dent Traumatol 1987; 3: 86-90.

Yoshida M, Fukushima H, Yamamoto K, et al. Correlation between clinical symptoms and microorganisms isolated from root canals of teeth with periapical pathosis. J Endod 1987; 13: 24-28.

\subsubsection{Gingivale Schmerzen}

Akintoye SO und Greenberg MS. Recurrent aphthous stomatitis. Dent Clin North Am 2014; 58: 281-297.

Al-Hashimi I, Schifter M, Lockhart PB, et al. Oral lichen planus and oral lichenoid lesions: diagnostic and therapeutic considerations. Oral Surg Oral Med Oral Pathol Oral Radiol Endod 2007; 103(suppl): S25.e1-12.

Andrade P, Brinca A und Goncalo M. Patch testing in fixed drug eruptions - a 20year review. Contact Dermatitis 2011; 65: 195-201.

Bascones-Martínez A, García-García V, Meurman JH, et al. Immune-mediated diseases: what can be found in the oral cavity? Int J Dermatol 2015; 54: 258-270.
Batchelor JM und Todd PM. Allergic contact stomatitis caused by a polyether dental impression material. Contact Dermatitis 2010; 63: 296-297.

Benedix F, Schilling M, Schaller M, et al. A young woman with recurrent vesicles on the lower lip: fixed drug eruption mimicking herpes simplex. Acta Derm Venereol 2008; 88: 491-494.

Calapai G, Miroddi M, Mannucci C, et al. Oral adverse reactions due to cinnamonflavoured chewing gums consumption. Oral Dis 2014; 20: 637-643.

Carrozzo M, Togliatto M und Gandolfo S. [Erythema multiforme. A heterogeneous pathologic phenotype]. Minerva Stomatol 1999; 48: 217-226.

Farthing P, Bagan JV and Scully C. Mucosal disease series. Number IV. Erythema multiforme. Oral Dis 2005; 11: 261-267.

Feller L, Altini M, Chandran R, et al. Noma (cancrum oris) in the South African context. J Oral Pathol Med 2014; 43: 1-6.

Firth FA, Friedlander LT, Parachuru VP, et al. Regulation of immune cells in oral lichen planus. Arch Dermatol Res 2015; 307: 333-339.

Fitzpatrick SG, Hirsch SA und Gordon SC. The malignant transformation of oral lichen planus and oral lichenoid lesions: a systematic review. J Am Dent Assoc 2014; 145: 45-56.

Fourie J and Boy SC. Oral mucosal ulceration - a clinician's guide to diagnosis and treatment. S Afr Dent J 2016; 71: 500-508.

Fukiwake N, Moroi Y, Urabe K, et al. Detection of autoantibodies to desmoplakin in a patient with oral erythema multiforme. Eur J Dermatol 2007; 17: 238-241.

Gondivkar SM, Gadbail A und Chole R. Oral pregnancy tumor. Contemp Clin Dent 2010; 1: 190-192. Gorouhi F, Davari P und Fazel N. Cutaneous and mucosal lichen planus: a comprehensive review of clinical subtypes, risk factors, diagnosis, and prognosis. ScientificWorldJournal 2014; 2014: 742826.

Gupta S, Gupta S, Mittal A, et al. Oral fixed drug eruption caused by gabapentin. J Eur Acad Dermatol Venereol 2009; 23: 1207-1208.

Harman KE, Albert S und Black MM. Guidelines for the management of pemphigus vulgaris. Br J Dermatol 2003; 149: 926-937.

Hasan S. Desquamative gingivitis - a clinical sign in mucous membrane pemphigoid: report of a case and review of literature. J Pharm Bioallied Sci 2014; 6: 122-126.

Hertel M, Matter D, Schmidt-Westhausen AM, et al. Oral syphilis: a series of 5 cases. J Oral Maxillofac Surg 2014; 72: 338-345.

Jain P and Jain I. Oral manifestations of tuberculosis: step towards early diagnosis. J Clin Diagn Res 2014; 8: ZE18-21.

Kay LW. Investigations into the nature of pericoronitis. Br J Oral Surg 1966; 3: 188-205.

Khatibi M, Shakoorpour AH, Jahromi ZM, et al. The prevalence of oral mucosal lesions and related factors in 188 patients with systemic lupus erythematosus. Lupus 2012; 21: 1312-1315.

Kind F, Scherer K und Bircher AJ. Allergic contact stomatitis to cinnamon in chewing gum mistaken as facial angioedema. Allergy 2010; 65: 276-277.

Perusquia-Ortiz AM, Vazquez-Gonzalez D und Bonifaz A. Opportunistic filamentous mycoses: aspergillosis, mucormycosis, phaeohyphomycosis and hyalohyphomycosis. J Dtsch Dermatol Ges 2012; 10: 611-621.

Raber-Durlacher JE, Elad S und Barasch A. Oral mucositis. Oral Oncol 2010; 46: 452-456.

Rosenthal DI und Trotti A. Strategies for managing radiation-induced mucositis in head and neck cancer. Semin Radiat Oncol 2009; 19: 29-34.

Savin JA. Current causes of fixed drug eruption in the UK. Br J Dermato/ 2001; 145 667-668.

Scully C. Clinical practice. Aphthous ulceration. N Engl J Med 2006; 355: 165-172.

Shiboski CH, Patton LL, Webster-Cyriaque JY, et al. The Oral HIV/AIDS Research Alliance: updated case definitions of oral disease endpoints. J Oral Pathol Med 2009; 38: 481-488

Slebioda Z, Szponar E und Kowalska A. Recurrent aphthous stomatitis: genetic aspects of etiology. Postepy Dermatol Alergol 2013; 30: 96-102.

Theander E und Jacobsson LT. Relationship of Sjögren's syndrome to other connective tissue and autoimmune disorders. Rheum Dis Clin North Am 2008; 34: 935-947.

Van der Waal I. Oral potentially malignant disorders: is malignant transformation predictable and preventable? Med Oral Patol Oral Cir Bucal 2014; 19: e386-390.

Venables ZC, Narayana K und Johnston GA. Two unusual cases of allergic contact stomatitis caused by methacrylates. Contact Dermatitis 2016; 74: 126-127.

Von Arx DP und Husain A. Oral tuberculosis. Br Dent J 2001; 190: 420-422.

Warnakulasuriya S, Johnson NW und van der Waal I. Nomenclature and classification of potentially malignant disorders of the oral mucosa. J Oral Pathol Med 2007; 36: 575-580.

Yuan A and Woo SB. Adverse drug events in the oral cavity. Oral Surg Oral Med Oral Pathol Oral Radiol 2015; 119: 35-47. 


\subsubsection{Schmerzen in der Mundschleimhaut}

Abdalla-Aslan R, Benoliel R, Sharav Y, et al. Characterization of pain originating from oral mucosal lesions. Oral Surg Oral Med Oral Pathol Oral Radiol 2016; 121 : 255-261.

Akintoye SO und Greenberg MS. Recurrent aphthous stomatitis. Dent Clin North Am 2014; 58: 281-297.

Al-Hashimi I, Schifter M, Lockhart PB, et al. Oral lichen planus and oral lichenoid lesions: diagnostic and therapeutic considerations. Oral Surg Oral Med Oral Pathol Oral Radiol Endod 2007; 103(suppl): S25.e1-12.

Andrade P, Brinca A und Goncalo M. Patch testing in fixed drug eruptions - a 20year review. Contact Dermatitis 2011; 65: 195-201.

Bascones-Martínez A, García-García V, Meurman JH, et al. Immune-mediated diseases: what can be found in the oral cavity? Int J Dermatol 2015; 54: 258-270.

Batchelor JM und Todd PM. Allergic contact stomatitis caused by a polyether dental impression material. Contact Dermatitis 2010; 63: 296-297.

Benedix $F$, Schilling $M$, Schaller $M$, et al. A young woman with recurrent vesicles on the lower lip: fixed drug eruption mimicking herpes simplex. Acta Derm Venereol 2008; 88: 491-494.

Calapai G, Miroddi M, Mannucci C, et al. Oral adverse reactions due to cinnamonflavoured chewing gums consumption. Oral Dis 2014; 20: 637-643.

Carrozzo M, Togliatto M und Gandolfo S. Erythema multiforme. A heterogeneous pathologic phenotype. Minerva Stomatol 1999; 48: 217-226.

Duncan GG, Epstein JB, Tu D, et al. Quality of life, mucositis, and xerostomia from radiotherapy for head and neck cancers: a report from the NCIC CTG HN2 randomized trial of an antimicrobial lozenge to prevent mucositis. Head Neck 2005; 27: 421-428.

Farthing P, Bagan JV und Scully C. Mucosal disease series. Number IV. Erythema multiforme. Oral Dis 2005; 11: 261-267.

Firth FA, Friedlander LT, Parachuru VP, et al. Regulation of immune cells in oral lichen planus. Arch Dermatol Res 2015; 307: 333-339.

Fitzpatrick SG, Hirsch SA und Gordon SC. The malignant transformation of oral lichen planus and oral lichenoid lesions: a systematic review. J Am Dent Assoc 2014; 145: 45-56.

Fourie J and Boy SC. Oral mucosal ulceration - a clinician's guide to diagnosis and treatment. S Afr Dent J 2016; 71: 500-508.

Fukiwake N, Moroi Y, Urabe K, et al. Detection of autoantibodies to desmoplakin in a patient with oral erythema multiforme. Eur J Dermatol 2007; 17: 238-241.

Gorouhi F, Davari P und Fazel N. Cutaneous and mucosal lichen planus: a comprehensive review of clinical subtypes, risk factors, diagnosis, and prognosis. ScientificWorldJournal 2014; 2014:742826.

Gupta S, Gupta S, Mittal A, et al. Oral fixed drug eruption caused by gabapentin. J Eur Acad Dermatol Venereol 2009; 23: 1207-1208.

Harman KE, Albert $S$ and Black MM. Guidelines for the management of pemphigus vulgaris. Br J Dermatol 2003; 149: 926-937.

Hertel M, Matter D, Schmidt-Westhausen AM, et al. Oral syphilis: a series of 5 cases. J Oral Maxillofac Surg 2014; 72: 338-345.

Jain P und Jain I. Oral manifestations of tuberculosis: step towards early diagnosis. J Clin Diagn Res 2014; 8: ZE18-21.

Khatibi M, Shakoorpour AH, Jahromi ZM, et al. The prevalence of oral mucosa lesions and related factors in 188 patients with systemic lupus erythematosus. Lupus 2012; 21: 1312-1315.

Kind F, Scherer K und Bircher AJ. Allergic contact stomatitis to cinnamon in chewing gum mistaken as facial angioedema. Allergy 2010; 65: 276-277.

Mravak-Stipetić M. Differential diagnostics of painful conditions of oral mucosa anticancer therapy. Rad 507. Med Sci 34: 55-73.

Perusquia-Ortiz AM, Vazquez-Gonzalez D und Bonifaz A. Opportunistic filamentous mycoses: aspergillosis, mucormycosis, phaeohyphomycosis and hyalohyphomycosis. J Dtsch Dermatol Ges 2012; 10: 611-621.

Raber-Durlacher JE, Elad S und Barasch A. Oral mucositis. Oral Oncol 2010; 46: 452-456.

Rosenthal DI and Trotti A. Strategies for managing radiation-induced mucositis in head and neck cancer. Semin Radiat Oncol 2009; 19: 29-34.

Saccucci M, Di Carlo G, Bossu' M, et al. Autoimmune diseases and their manifestations on oral cavity: diagnosis and clinical management. J Immunol Res 2018; 2018: 6061825.

Savin JA. Current causes of fixed drug eruption in the UK. Br J Dermatol 2001; 145 : 667-668.

Scully C. ABC of oral health: mouth ulcers and eine andere Ursaches of orofacial soreness and pain. Br Med J 2000; 321: 162-165.

Scully C. Clinical practice. Aphthous ulceration. N Engl J Med 2006; 355: 165-172.
Shiboski CH, Patton LL, Webster-Cyriaque JY, et al. The Oral HIV/AIDS Research Alliance: updated case definitions of oral disease endpoints. J Oral Pathol Med 2009; 38: 481-488.

Slebioda Z, Szponar E und Kowalska A. Recurrent aphthous stomatitis: genetic aspects of etiology. Postepy Dermatol Alergol 2013; 30: 96-102.

Sonis ST, Elting LS, Keefe D, et al. Perspectives on cancer therapy-induced mucosal injury: pathogenesis, measurement, epidemiology, and consequences for patients. Cancer 2004: 100(9 suppl): 1995-2025.

Theander E und Jacobsson LT. Relationship of Sjögren's syndrome to other connective tissue and autoimmune disorders. Rheum Dis Clin North Am 2008; 34 : 935-947.

Treister N und Sonis S. Mucositis: biology and management. Curr Opin Otolaryngol Head Neck Surg 2007; 15: 123-129.

Van der Waal I. Oral potentially malignant disorders: is malignant transformation predictable and preventable? Med Oral Patol Oral Cir Bucal 2014; 19: e386-390.

Venables ZC, Narayana K und Johnston GA. Two unusual cases of allergic contact stomatitis caused by methacrylates. Contact Dermatitis 2016; 74: 126-127.

Vera-Llonch M, Oster G, Ford CM, et al. Oral mucositis and outcomes of allogeneic hematopoietic stem-cell transplantation in patients with hematologic malignancies. Support Care Cancer 2007; 15: 491-496.

Von Arx DP und Husain A. Oral tuberculosis. Br Dent J 2001; 190: 420-422.

Warnakulasuriya S, Johnson NW und van der Waal I. Nomenclature and classification of potentially malignant disorders of the oral mucosa. J Oral Pathol Med 2007; 36: 575-580.

Yuan A und Woo SB. Adverse drug events in the oral cavity. Oral Surg Oral Med Oral Pathol Oral Radiol 2015; 119: 35-47.

\subsubsection{Schmerzen der Speicheldrüse}

Bassim CW, Fassil H, Mays JW, et al. Oral disease profiles in chronic graft versus host disease. J Dent Res 2015; 94: 547-554.

Brook I. The bacteriology of salivary gland infections. Oral Maxillofac Surg Clin North Am 2009; 21: 269-274.

Guzzo M, Locati LD, Prott FJ, et al. Major and minor salivary gland tumors. Crit Rev Oncol Hematol 2010; 74: 134-148.

Klein Hesselink EN, Brouwers AH, de Jong JR, et al. Effects of radioiodine treatment on salivary gland function in patients with differentiated thyroid carcinoma: a prospective study. J Nucl Med 2016; 57: 1685-1691.

Leerdam CM, Martin HC und Isaacs D. Recurrent parotitis of childhood. J Paediatr Child Health 2005; 41: 631-634.

Napeñas JJ und Rouleau TS. Oral complications of Sjögren's syndrome. Oral Maxillofac Surg Clin North Am 2014; 26: 55-56.

Shiboski SC, Shiboski CH, Criswell L, et al. American College of Rheumatology classification criteria for Sjögren's syndrome: a data-driven, expert consensus approach in the Sjögren's International Collaborative Clinical Alliance cohort. Arthritis Care Res (Hoboken) 2012; 64: 475-487.

Wilson KF, Meier JD und Ward PD. Salivary gland disorders. Am Fam Physician 2014; 89: 882-888.

\subsubsection{Schmerzen im Kieferknochen}

Annibali S, Cristalli MP, Solidani M, et al. Langerhans cell histiocytosis: oral/periodontal involvement in adult patients. Oral Dis 2009; 15: 596-601.

Campanacci M, Baldini N, Boriani S, et al. Giant-cell tumor of bone. J Bone Joint Surg Am 1987; 69: 106-114.

Caparrotti F, Huang SH, Lu L, et al. Osteoradionecrosis of the mandible in patients with oropharyngeal carcinoma treated with intensitymodulated radiotherapy. Cancer 2017; 123: 3691-3700.

Caputo ND, Raja A, Shields C, et al. Re-evaluating the diagnostic accuracy of the tongue blade test: still useful as a screening tool for mandibular fractures? J Emerg Med 2013; 45: 8-12.

Chopra S, Kamdar D, Ugur OE, et al. Factors predictive of severity of osteoradionecrosis of the mandible. Head Neck 2011; 33: 1600-1605.

Copley L und Dormans JP. Benign pediatric bone tumors. Evaluation and treatment. Pediatr Clin North Am 1996; 43: 949-966.

Da Fonseca M, Oueis HS und Casamassimo PS. Sickle cell anemia: a review for the pediatric dentist. Pediatr Dent 2007; 29: 159-169.

Daly B, Sharif MO, Newton T, et al. Local interventions for the management of alveolar osteitis (dry socket). Cochrane Database Syst Rev 2012; 12: CD006968. 
D'Ambrosio N, Soohoo S, Warshall C, et al. Craniofacial and intracranial manifestations of langerhans cell histiocytosis: report of findings in 100 patients. AJR Am J Roentgenol 2008; 191: 589-597. Erdmann D, Follmar KE, Debruijn M, et al. A retrospective analysis of facial fracture etiologies. Ann Plast Surg 2008; 60: 398-403.

Gabrielli E, Fothergill AW, Brescini L, et al. Osteomyelitis caused by Aspergillus species: a review of 310 reported cases. Clin Microbiol Infect 2014; 20: 559-565.

Grabowski GA. Recent clinical progress in Gaucher disease. Curr Opin Pediatr 2005; 17: 519-524.

Grabowski GA, Andria G, Baldellou A, et al. Pediatric non-neuronopathic Gaucher disease: presentation, diagnosis and assessment. Consensus statements. Eur J Pediatr 2004; 163: 58-66.

Greenspan A. Benign bone-forming lesions: osteoma, osteoid osteoma, and osteoblastoma. Clinical, imaging, pathologic, and differential considerations. Skeletal Radiol 1993; 22: 485-500.

Gupta S, Sreenivasan V, Patil PB. Dental complications of herpes zoster: two case reports and review of literature. Indian J Dent Res 2015; 26: 214-219.

Jones AC, Prihoda TJ, Kacher JE, et al. Osteoblastoma of the maxilla and mandible: a report of 24 cases, review of the literature, and discussion of its relationship to osteoid osteoma of the jaws. Oral Surg Oral Med Oral Pathol Oral Radiol Endod 2006; 102: 639-650.

Kyle RA, Gertz MA, Witzig TE, et al. Review of 1027 patients with newly diagnosed multiple myeloma. Mayo Clin Proc 2003; 78: 21-33.

Mirabello L, Troisi RJ, Savage SA. Osteosarcoma incidence and survival rates from 1973 to 2004: data from the Surveillance, Epidemiology, and End Results Program. Cancer 2009; 115: 1531-1543.

Nilesh K und Vande AV. Mucormycosis of maxilla following tooth extraction in immunocompetent patients: Reports and review. J Clin Exp Dent 2018; 10: e300-e305.

Platt OS, Thorington BD, Brambilla DJ, et al. Pain in sickle cell disease. Rates and risk factors. NEngl J Med 1991; 325: 11-16.

Prasad KC, Prasad SC, Mouli N, et al. Osteomyelitis in the head and neck. Acta Otolaryngol 2007; 127: 194-205.

Ruggiero SL, Dodson TB, Fantasia J, et al.; American Association of Oral and Maxillofacial Surgeons. American Association of Oral and Maxillofacial Surgeons position paper on medication-related osteonecrosis of the jaw-2014 update. J Oral Maxillofac Surg 2014; 72: 1938-1956.

Sharif MO, Dawoud BE, Tsichlaki A, et al. Interventions for the prevention of trockenes Zahnfach: an evidence-based update. Br Dent J 2014; 217: 27-30. Van Staa TP, Selby P, Leufkens HG, et al. Incidence and natural history of Paget's disease of bone in England and Wales. J Bone Miner Res 2002; 17: 465-471.

Wyers MR. Evaluation of pediatric bone lesions. Pediatr Radiol 2010; 40: 468-473.

Yildiz C, Erler K, Atesalp AS, et al. Benign bone tumors in children. Curr Opin Pediatr 2003; 15: 58-67.

Zou $\mathrm{H}$, Yang $\mathrm{H}$, Zou Y, et al. Primary diffuse large B-cell lymphoma in the maxilla: A case report. Medicine (Baltimore) 2018; 97: e10707.

\section{Myofaszial-orofaziale Schmerzen}

Allgemeine Kommentare: Temporomandibuläre Dysfunktion (TMD) ist ein Begriff, mit dem eine ganze Reihe schmerzhafter und nichtschmerzhafter Störungen beschrieben wird, die die Kaumuskulatur, das Kiefergelenk und benachbarte Strukturen betreffen. Die von INfORM publizierten diagnostischen Kriterien für eine temporomandibuläre Dysfunktion (Diagnostic Criteria for Temporomandibular Disorders, DC/TMD) sind zuverlässig und allgemein anerkannt. Nichtsdestotrotz bestehen weiter Kontroversen im Hinblick auf die Terminologie bezüglich chronischer Muskelschmerzen. Während die DC/TMD „Myalgie" und "myofasziale Schmerzen" verwenden, wurden hierzu bereits auch andere Vorschläge gemacht, so zum Beispiel „persistierende orofaziale Muskelschmerzen“. Die ICOP benutzt als Oberbegriff „myofaszial-orofaziale Schmerzen“ und bleibt dabei trotz des Fehlens konkreter Nachweise für eine Verbindung von Schmerzen mit spezifischen Muskelstrukturen oder -geweben beim Begriff „myofaszial“'. Ferner wird mit Blick auf die Übereinstim- mung mit den DC/TMD-Kriterien der Begriff „temporomandibuläre Dysfunktion" beibehalten.

Die zeitliche Unterscheidung myofaszialer Schmerzen in der ICOP ist neu (und findet sich nicht in den DC/TMD-Kriterien), richtet sich aber nach den allgemeinen Prinzipien aus der ICHD-3 für 2. Kopfschmerz vom Spannungstyp: das heißt, es wurden ähnliche Kriterien hinsichtlich der Häufigkeit der Schmerzepisoden eingeführt. Zwar mag man sich über die klinische Signifikanz myofaszialer Gelegenheitsschmerzen (seltener als einmal pro Monat auftretend) streiten, doch scheint es solide Erkenntnisse zu geben, die für deren Abgrenzung von häufiger auftretenden Schmerzen sprechen. Zukünftige Studien auf Grundlage der vorgeschlagenen zeitlichen Unterscheidung der myofaszialen Schmerzen könnten therapeutische Implikationen offenbaren.

Obwohl einige Zahnärzte und viele mit orofazialen Schmerzen befasste Spezialisten bei ihrem Schmerzmanagement zusätzlich zu Schmerzen in der Kiefermuskulatur Schmerzen in der Halsmuskulatur einbeziehen mögen, wurde beschlossen, in diesem Stadium keine Klassifikation für Schmerzen in der Halsmuskulatur einzubeziehen. Ein Vorschlag wäre jedoch, derartige Schmerzen unter Einhaltung ähnlicher Prinzipien (d. h. nach Häufigkeit des Schmerzauftritts, lokalen Untersuchungsbefunden und mit oder ohne Schmerzübertragung) zu klassifizieren. Das gleiche Prinzip ließe sich auf andere Muskeln in der orofazialen Region anwenden (z. B. die Zungen- oder Schluckmuskulatur).

Im Hinblick auf Schmerzübertragung wird berücksichtigt, dass akute und chronische Schmerzen in der orofazialen Muskulatur mit diesem klinischen Phänomen einhergehen können (d.h., der Schmerz wird an einer anderen Stelle als der wahrgenommen, wo der nozizeptive oder schädliche Reiz auftrat). Die pathophysiologische Bedeutung dieses Phänomens bleibt ebenso unklar wie die therapeutischen Implikationen. Aus diagnostischer Sicht jedoch bleibt es wichtig, übertragene Schmerzen von lokalen Schmerzen zu unterscheiden. Dementsprechend werden sämtliche Diagnosen von myofaszialer Schmerzen nach dem Vorliegen oder dem Fehlen einer Schmerzübertragung bei der Tastuntersuchung in Unterkategorien unterschieden. Die Definition von „Schmerzübertragung“ folgt der in den DC/TMD-Kriterien.

Die DC/TMD-Kriterien operieren mit den Begriffen des projizierten Schmerzes, bei welchem der Schmerz, anders als beim übertragenen Schmerz, innerhalb der Grenzen der betroffenen anatomischen Struktur bleibt. Zu Forschungszwecken können bei Bedarf spezifische Kriterien für myofasziale Schmerzen mit Schmerzausstrahlung gemäß der DC/TMD-Kriterien angewendet werden.

Weiter ist anzumerken, dass die DC/TMD-Kriterien sich nicht allein auf den M. temporalis und M. masseter beschränken. Die Untersuchungskriterien spezifizieren vielmehr diese Muskeln deshalb, weil (a) hier eine höhere Reliabilität der Untersuchung gegeben ist und (b) nahezu alle Personen mit schmerzhafter myofaszialer temporomandibulärer Dysfunktion Schmerzen in wenigstens einem von beiden aufweisen. Daraus folgt jedoch nicht zwingend, dass sich positive Zeichen nicht in der übrigen Kaumuskulatur finden lassen. Die Beschränkung auf den M. temporalis und M. masseter bedeutet nicht, dass Personen, die vielleicht eine streng lokalisierte Myalgie in anderen Kaumuskeln aufweisen, ausgeschlossen werden, und für die klinische Praxis mag dies auch wie eine unnöti- 
ge Einschränkung wirken. Bei zukünftigen Überarbeitungen könnte die ICOP-Klassifikation also gegebenenfalls weitere orofaziale Muskeln einbeziehen.

Ein zentrales Kriterium bei der Diagnose von Muskelschmerzen gemäß der DC/TMD-Kriterien besteht darin, dass die Patienten beim Provokationstest von Schmerzen berichten: standardisierte Palpation und/oder Schmerzen beim Öffnen des Kiefers. Dies spiegelt sich in den ICOP-Kriterien wider. In mehreren Untersuchungen befasst man sich mit zusätzlichen dynamischen/statischen Tests der Kiefermuskulatur und des Kiefergelenks zur Erhöhung der diagnostischen Sensitivität und Spezifität. Es bedarf noch weiterer Forschung, bevor eine Empfehlung für die Einbeziehung derartiger diagnostischer Tests ausgesprochen werden kann. Die konkreten Anweisungen und Kriterien für einen positiven Befund bei Provokationstests sollten sich an die DC/TMD-Beschreibungen halten, wobei die Wichtigkeit einer Standardisierung des Kraftaufwands bei der Palpation und deren Dauer zu betonen ist: $1 \mathrm{~kg}$ für 2 Sekunden, um eine Schmerzprovokation bei der Tastuntersuchung zu ermitteln, und $1 \mathrm{~kg}$ für 5 Sekunden, um Schmerzübertragungen (oder eine Schmerzausbreitung) zu ermitteln. Es gilt zu beachten, dass es zwar für die Palpationskräfte umfangreiche empirische Belege im Hinblick auf die Unterscheidung zwischen Fällen und NichtFällen gibt, die Dauer der Palpation jedoch nur vorläufig empirisch bestätigt ist.

Gegenüberstellung primärer und sekundärer Schmerzen: Basierend auf der Klassifikation chronischer Schmerzen der International Association for the Study of Pain (IASP) wird in diesem Kapitel zwischen primären und sekundären Schmerzen unterschieden. Bei primären Schmerzen lässt sich die konkrete Ätiologie oder Ursache nicht ermitteln - das heißt, sie sind idiopathischer Art, obwohl im Hinblick auf ihre pathophysiologischen Mechanismen durchaus umfangreiches Wissen vorhanden sein kann. Bei sekundären Schmerzen ist der Schmerz sekundäre Folge eines anderen Krankheitsbildes oder einer anderen Ursache.

Im Hinblick auf die Grunderkrankungen (Tendonitis, Myositis und Muskelspasmus) hinter sekundären myofaszialen Schmerzen werden die Definitionen der Expanded Taxonomy for Temporomandibular Disorders verwendet. Es sind Kriterienvorschläge für weitere Forschung, die noch nicht validiert wurden.

\subsection{Primäre myofaszial-orofaziale Schmerzen}

Beschreibung: Schmerzen in der Kaumuskulatur mit oder ohne funktionelle Beeinträchtigung, die keiner anderen Störung zugeschrieben werden können.

\section{Diagnostische Kriterien:}

A. Myofasziale Schmerzen, die die Kriterien B-D erfüllen

B. In einer oder mehreren Episoden ${ }^{1}$ auftretend oder nicht remittierend

C. Auftreten im Kiefer, an der Schläfe, am Ohr und/oder vor dem Ohr bei Vorliegen der beiden folgenden Faktoren:

1. Bestätigung bei Untersuchung der Stelle(n) im M. temporalis und/oder M. masseter einseitig oder beidseits

2. Provoziert durch eines oder beides von Folgendem: a) Palpation des M. temporalis und/oder M. masseter einseitig oder beidseits

b) Maximale eigenständige oder unterstützte Kieferöffnungsbewegung(en)

D. Modifiziert ${ }^{2}$ durch Kieferbewegungen, -funktion oder Parafunktion (d.h. Zähneknirschen oder Zusammenbeißen der Zähne)

E. Nicht besser erklärt durch eine andere ICOP-Diagnose

\section{Anmerkungen:}

1. Die Episoden können einmalig sein oder innerhalb eines Tages wiederkehren, wobei jede mindestens 30 Minuten anhält und innerhalb des Tages eine Gesamtdauer von mindestens 2 Stunden aufweist.

2. Der Schmerz kann zu- oder abnehmen

\subsubsection{Akute primäre myofaszial-orofaziale Schmerzen}

Beschreibung: Leichte bis mittelstarke tiefe, ziehende oder drückende Schmerzen in der Kaumuskulatur, episodisch oder anhaltend, oft verbunden mit funktioneller Beeinträchtigung wie etwa beobachtete Schwierigkeiten beim Bewegen des Unterkiefers, Kauen und/oder Gähnen etc., und mit Schmerzbeginn innerhalb der letzten 3 Monate.

\section{Diagnostische Kriterien:}

A. Myofasziale Schmerzen, die die Kriterien für 2.1 Primäre myofaszial-orofaziale Schmerzen sowie Kriterium B erfüllen

B. Beginn innerhalb der letzten 3 Monate

\subsubsection{Chronische primäre myofaszial-orofaziale} Schmerzen

Beschreibung: Leichte bis mittelstarke tiefe, ziehende oder drückende Schmerzen in der Kaumuskulatur, episodisch oder ununterbrochen, oft verbunden mit funktioneller Beeinträchtigung wie etwa beobachtete Schwierigkeiten beim Bewegen des Unterkiefers, Kauen und/oder Gähnen etc., und mit Schmerzbeginn vor mehr als 3 Monaten. Oft mit psychosozialen Belastungen assoziiert.

\section{Diagnostische Kriterien:}

A. Myofasziale Schmerzen, die die Kriterien für 2.1 Primäre myofaszial-orofaziale Schmerzen sowie Kriterium B und C erfüllen

B. Beginn vor $>3$ Monaten

C. In mindestens 10 Episoden oder als Dauerschmerz auftretend

\subsubsection{Chronischer primärer myofaszial-orofazialer Gelegenheits-} schmerz

\section{Diagnostische Kriterien:}

A. Myofasziale Schmerzen, die die Kriterien für 2.1.2 Chronische primäre myofaszial-orofaziale Schmerzen sowie Kriterium B erfüllen

B. Treten an $<1$ Tag/Monat auf

\subsubsection{Chronischehäufige primäre myofaszial-orofaziale Schmerzen} Diagnostische Kriterien:

A. Myofasziale Schmerzen, die die Kriterien für 2.1.2 Chronische primäre myofaszial-orofaziale Schmerzen sowie Kriterium B erfüllen 
B. Treten im Durchschnitt $>3$ Monate an 1-14 Tagen monatlich auf ( $>12$ und $<180$ Tage/Jahr)

2.1.2.2.1 Chronische häufige primäre myofaszial-orofaziale Schmerzen ohne Schmerzübertragung

\section{Diagnostische Kriterien:}

A. Myofasziale Schmerzen, die die Kriterien für 2.1.2.2 Chronische häufige primäre myofaszial-orofaziale Schmerzen sowie Kriterium $B$ erfüllen

B. Kein Bericht über Schmerzen an einer Stelle jenseits der Grenzen des gerade palpierten Muskels (M. temporalis oder $M$. masseter)

2.1.2.2.2 Chronische häufige primäre myofaszial-orofaziale Schmerzen mit Schmerzübertragung

\section{Diagnostische Kriterien:}

A. Myofasziale Schmerzen, die die Kriterien für 2.1.2.2 Chronische häufige primäre myofaszial-orofaziale Schmerzen sowie Kriterium $B$ erfüllen

B. Bericht über Schmerzen an einer Stelle jenseits der Grenzen des gerade palpierten Muskels ( $M$. temporalis oder M. masseter)

2.1.2.3 Chronische sehr häufige primäre myofaszial-orofaziale Schmerzen

\section{Diagnostische Kriterien:}

A. Myofasziale Schmerzen, die die Kriterien für 2.1.2 Chronische primäre myofaszial-orofaziale Schmerzen sowie Kriterium B erfüllen

B. Tritt an $>15$ Tagen/Monat durchschnittlich für $>3$ Monate auf (>180 Tage/Jahr)

2.1.2.3.1 Chronische sehr häufige primäre myofaszial-orofaziale Schmerzen ohne Schmerzübertragung

\section{Diagnostische Kriterien:}

A. Myofasziale Schmerzen, die die Kriterien für 2.1.2.3 Chronische sehr häufige primäre myofaszial-orofaziale Schmerzen sowie Kriterium B erfüllen

B. Kein Bericht über Schmerzen an einer Stelle jenseits der Grenzen des gerade palpierten Muskels (M. temporalis oder M. masseter)

2.1.2.3.2 Chronische sehr häufige primäre myofaszial-orofaziale Schmerzen mit Schmerzübertragung

\section{Diagnostische Kriterien:}

A. Myofasziale Schmerzen, die die Kriterien für 2.1.2.3 Chronische sehr häufige primäre myofaszial-orofaziale Schmerzen sowie Kriterium B erfüllen

B. Bericht über Schmerzen an einer Stelle jenseits der Grenzen des gerade palpierten Muskels (M. temporalis oder M. masseter)

\subsection{Sekundäre myofaszial-orofaziale Schmerzen}

Beschreibung: Myofasziale Schmerzen verursacht durch eine Grunderkrankung (Entzündung, Infektion oder Muskelspasmus).
Diagnostische Kriterien:

A. Myofasziale Schmerzen in einem Muskel des Kauapparats, die Kriterium $C$ und D erfüllen

B. Es wurde eine Grunderkrankung diagnostiziert, die bekanntermaßen myofasziale Schmerzen ${ }^{1}$ auslösen kann

C. Der myofasziale Schmerz weist beide der folgenden Charakteristika auf:

1. Auftreten im Kiefer, an der Schläfe, am Ohr und/oder vor dem Ohr bei Vorliegen der beiden folgenden Faktoren:

a) Bestätigung bei Untersuchung der Stelle(n) im/in den betroffenen Muskel( $n$ ) oder Sehne(n)

b) Provoziert durch Palpation der betroffenen Sehne(n) und/oder maximale eigenständige oder unterstützte Kieferöffnungsbewegung(en) ${ }^{2}$

2. Modifiziert ${ }^{3}$ durch Kieferbewegung, -funktion oder Parafunktion (z. B. Zähneknirschen oder Zusammenbeißen der Zähne)

D. Der ursächliche Zusammenhang wurde nachgewiesen ${ }^{4}$

E. Nicht besser erklärt durch eine andere ICOP-Diagnose

\section{Anmerkungen:}

1. Die Dysfunktion wird bei jeder Unterform spezifiziert.

2. Diese Zeichen lassen sich bei der körperlichen Untersuchung demonstrieren oder werden, bei Schmerzen die sich bereits wieder gelegt haben, anamnestisch berichtet.

3. Der Schmerz kann zu- oder abnehmen.

4. Der erforderliche Nachweis wird bei jeder Unterform spezifiziert.

\subsubsection{Myofaszial-orofaziale Schmerzen zurückzuführen} auf eine Tendinitis

Beschreibung: Von den Sehnen herrührender Schmerz, der durch Bewegungen, Funktionen oder Parafunktionen des Kiefers beeinflusst wird und sich durch Provokationstest der relevanten Kausehne replizieren lässt. Es kann eine Einschränkung der Unterkieferbewegung(en) als sekundäre Folge von Schmerzen vorliegen. Die Schläfensehne ist eine häufige Stelle für eine Sehnenentzündung und kann Schmerzen zu den Zähnen und anderen benachbarten Strukturen übertragen. Eine Tendinitis kann auch in anderen Sehnen der Kaumuskulatur auftreten.

\section{Diagnostische Kriterien:}

A. Myofasziale Schmerzen, die die Kriterien für 2.2 Sekundäre myofaszial-orofaziale Schmerzen sowie Kriterium C erfüllen

B. Es wurde eine Tendinitis eines oder mehrerer Kaumuskeln diagnostiziert

C. Der Nachweis des ursächlichen Zusammenhangs besteht aufgrund von mindestens zwei der folgenden Punkte:

1. Der myofasziale Schmerz hat sich in zeitlichem Zusammenhang mit dem Beginn der Tendinitis entwickelt

2. Der myofasziale Schmerz hat sich parallel zur Progression der Tendinitis signifikant ${ }^{1}$ verschlimmert

3. Der myofasziale Schmerz hat sich parallel zur Besserung oder dem Verschwinden der Tendinitis signifikant ${ }^{1}$ gebessert oder gelöst

D. Nicht besser erklärt durch eine andere ICOP-Diagnose 


\section{Anmerkung:}

1. Dergestalt, dass der Patient eine deutliche Veränderung der Intensität beschreibt.

\subsubsection{Myofaszial-orofaziale Schmerzen zurückzuführen auf eine Myositis}

Beschreibung: Durch eine Myositis verursachte Schmerzen, die einen oder mehrere Kaumuskeln betreffen, mit klinischen Charakteristika einer Entzündung oder Infektion: Ödeme, Erytheme und/ oder erhöhte Temperatur. In der Regel entstehen diese akut nach einem direkten Muskeltrauma oder durch eine Infektion oder bestehen chronisch bei einer Autoimmunerkrankung. Oft besteht als sekundäre Folge der Schmerzen eine Einschränkung eigenständiger Unterkieferbewegungen. Es kann zu einer Muskelverkalkung kommen (i.e. Myositis ossificans).

\section{Diagnostische Kriterien:}

A. Myofasziale Schmerzen, die die Kriterien für 2.2 Sekundäre myofaszial-orofaziale Schmerzen sowie Kriterium C erfüllen

B. Es wurde eine Myositis ${ }^{1}$ in einem oder mehreren Kaumuskeln diagnostiziert ${ }^{2,3}$

C. Der Nachweis des ursächlichen Zusammenhangs besteht aufgrund von mindestens zwei der folgenden Punkte:

1. Der myofasziale Schmerz hat sich in einem zeitlichen Zusammenhang mit dem Beginn der Myositis entwickelt

2. Der myofasziale Schmerz hat sich parallel zur Progression der Myositis signifikant $t^{4}$ verschlimmert

3. Der myofasziale Schmerz hat sich parallel zur Besserung oder dem Verschwinden der Myositis signifikant ${ }^{4}$ gebessert oder gelöst

D. Nicht besser erklärt durch eine andere ICOP-Diagnose

\section{Anmerkungen:}

1. Die Myositis kann auf eine Entzündung, eine Infektion oder ein Trauma zurückgehen.

2. Diagnostische Hinweise sind Ödeme, Erytheme und/oder eine erhöhte Temperatur über dem/den Muskel(n).

3. Serologische Tests ergeben einen erhöhten Enzymspiegel (z. B. Kreatin-Kinase), Entzündungsmarker und das Vorliegen von Autoimmunerkrankungen.

4. Dergestalt, dass der Patient eine deutliche Veränderung der Intensität beschreibt.

\subsubsection{Myofaszial-orofaziale Schmerzen zurückzuführen auf einen Muskelspasmus}

Beschreibung: Schmerzen verursacht durch eine plötzliche, unwillkürliche, reversible tonische Kontraktion eines Muskels. Derartige Spasmen können jeden der Kaumuskeln betreffen. Es kann eine akute Malokklusion vorliegen.

\section{Diagnostische Kriterien:}

A. Myofasziale Schmerzen, die die Kriterien für 2.2 Sekundäre myofaszial-orofaziale Schmerzen sowie Kriterium C erfüllen

B. Es wurde ein Muskelspasmus in einem oder mehreren Kaumuskeln diagnostiziert ${ }^{1,2}$

C. Der Nachweis des ursächlichen Zusammenhangs besteht aufgrund von mindestens zwei der folgenden Punkte:
1. Der myofasziale Schmerz hat sich in unmittelbarer zeitlicher Beziehung zum Beginn des Spasmus entwickelt

2. Der myofasziale Schmerz hat sich parallel zur Progression des Spasmus signifikant ${ }^{3}$ verschlimmert

3. Der myofasziale Schmerz hat sich parallel zur Besserung oder dem Verschwinden des Spasmus signifikant ${ }^{3}$ verbessert oder gelöst

D. Nicht besser erklärt durch eine andere ICOP-Diagnose

\section{Anmerkungen:}

1. Eine Bewegungseinschränkung des Kiefers in eine Richtung, die in der Verlängerung des/der betroffenen Muskels/Muskeln liegt, hat diagnostischen Wert: Zum Beispiel ist die Öffnung für Kieferschlussmuskeln auf $<40 \mathrm{~mm}$ begrenzt; für den $\mathrm{M}$. pterygoideus lateralis ist die ipsilaterale Bewegung auf $<7 \mathrm{~mm}$ beschränkt.

2. Falls die Diagnose bestätigt werden muss, zeigt die intramuskuläre Elektromyographie (EMG) im Vergleich zu einem kontralateral nicht betroffenen Muskel eine erhöhte Aktivität.

3. Dergestalt, dass der Patient eine deutliche Veränderung der Intensität beschreibt.

\section{Literatur}

Benoliel R, Svensson P, Heir G, et al. Persistent orofacial muscle pain. Oral Dis 2011; 17: 23-41.

Koutris M, Visscher CM, Lobbezoo F, et al. Comorbidity negatively influences the outcomes of diagnostic tests for musculoskeletal pain in the orofacial region. Pain 2013; 154: 927-932.

Masuda M, lida T, Exposto FG, et al. Referred pain and sensations evoked by standardized palpation of the masseter muscle in healthy participants. J Oral Facial Pain Headache 2018; 32: 159-166.

Osiewicz MA, Manfredini D, Loster BW, et al. Comparison of the outcomes of dynamic/static tests and palpation tests in TMD-pain patients. J Oral Rehabil 2018; 45: 185-190.

Peck CC, Goulet JP, Lobbezoo F, et al. Expanding the taxonomy of the diagnostic criteria for temporomandibular disorders. J Oral Rehabil 2014; 41:2-23.

Schiffman E, Ohrbach R, Truelove E, et al. Diagnostic Criteria for Temporomandibular Disorders (DC/TMD) for clinical and research applications: recommendations of the International RDC/TMD Consortium Network and Orofacial Pain Special Interest Group. J Oral Facial Pain Headache 2014; 28: 6-27.

Svensson P, Michelotti A, Lobbezoo F, et al. The many faces of persistent orofacial muscle pain. J Oral Facial Pain Headache 2015; 29: 207-208.

Treede RD, RiefW, Barke A, et al. A classification of chronic pain for ICD-11. Pain 2015; 156: 1003-1007.

Visscher CM, Lobbezoo F and Naeije M. A reliability study of dynamic and static pain tests in temporomandibular disorder patients. J Orofac Pain 2007; 21: 39-45.

Visscher CM, Naeije M, De Laat A, et al. Diagnostic accuracy of temporomandibular disorder pain tests: a multicenter study. J Orofac Pain 2009; 23: 108-114.

\section{Kiefergelenksschmerzen}

Allgemeine Kommentare: Wie die myofaszialen Schmerzen wurden auch die Kiefergelenksschmerzen basierend auf der Klassifikation chronischer Schmerzzustände der International Association for the Study of Pain (IASP) in primäre und sekundäre Subtypen untergliedert. Bei primären Schmerzen lässt sich die konkrete Ätiologie oder Ursache nicht ermitteln (d.h., sie sind idiopathischer Art), obwohl über ihre pathophysiologischen Mechanismen umfangreiches Wissen vorhanden sein kann. Bei sekundären Schmerzen ist 
der Schmerz sekundäre Folge eines anderen Krankheitsbildes oder einer anderen Ursache.

Was die Grunderkrankungen hinter sekundären Kiefergelenksschmerzen anbelangt, wird auf die Expanded Taxonomy for Temporomandibular Disorders und die Definitionen im Artikel von Alstergren et al. zur klinischen Diagnose einer Arthritis des Kiefergelenks zurückgegriffen. Hierzu zählen Arthritis, Diskusverlagerungen, eine degenerative Gelenkerkrankung sowie Subluxation. Diese Krankheitsbilder können jeweils verschiedene und spezifische Behandlungen erfordern, so dass es wichtig ist, den Grund für die auftretenden Kiefergelenksschmerzen zu kennen. Es gibt zudem noch weitere Krankheitsbilder, die zu Kiefergelenksschmerzen beitragen können - etwa generalisierte Schmerzzustände, bei denen das Gewebe am Kiefergelenk und in dessen Umgebung besonders sensibilisiert ist. Dies ist im Rahmen künftiger Studien näher zu betrachten.

Generell wird bei der Festlegung der nachfolgenden Kriterien der Begriff „zurückzuführen auf “ bevorzugt statt, verursacht durch“. "Verursacht durch“ impliziert einen erwiesenen kausalen Zusammenhang, der jedoch schwer zu beweisen sein mag. Außerdem kann die Beziehung in beide Richtungen ablaufen. „Zurückzuführen auf" beinhaltet hingegen die vorsichtigere Aussage, dass auf Grundlage der verfügbaren Daten die Annahme besteht, dass hier ein ursächlicher Zusammenhang besteht.

Die zeitliche Unterscheidung bei Schmerzen im Kiefer in der ICOP ist neu. Sie findet sich nicht in den Diagnostic Criteria for Temporomandibular Disorders (DC/TMD), sondern richtet sich nach den allgemeinen Prinzipien aus der ICHD-3 für 2. Kopfschmerz vom Spannungstyp. Das heißt, es wurden ähnliche Kriterien hinsichtlich der Häufigkeit der Schmerzepisoden eingeführt. Zwar mag man sich über die klinische Signifikanz von Gelegenheitsschmerzen (seltener als einmal pro Monat auftretend) im Kiefergelenk streiten, doch scheint es solide Erkenntnisse zu geben, die für deren Abgrenzung von häufiger auftretenden Schmerzzuständen sprechen. Zukünftige Studien auf Grundlage der vorgeschlagenen zeitlichen Unterscheidung der Kiefergelenksschmerzen könnten therapeutische Implikationen offenbaren.

Durch die Einbeziehung von Unterformen der Kiefergelenksschmerzen mit oder ohne Schmerzübertragung bleibt der Aufbau der Klassifikation der gleiche wie bei myofaszialen Schmerzen. Was die Schmerzübertragung anbelangt, wird weithin berücksichtigt, dass dieses klinische Phänomen bei akuten wie auch chronischen Kiefergelenksschmerzen auftreten kann (d.h., der Schmerz wird an einer anderen Stelle als der wahrgenommen, wo der nozizeptive Reiz oder Schadreiz auftrat). Die pathophysiologische Bedeutung dessen bleibt unklar, ebenso die therapeutischen Implikationen. Aus diagnostischer Sicht jedoch bleibt es wichtig, übertragene Schmerzen von lokalen Schmerzen zu unterscheiden. Dementsprechend wird bei Kiefergelenksschmerzen je nach Vorliegen oder Fehlen einer Schmerzübertragung bei der Tastuntersuchung in Unterkategorien unterschieden. Zukünftige Studien mögen zeigen, ob diese Untergliederung sinnvoll ist.

Die DC/TMD-Kriterien operieren zudem mit der Kategorie der Schmerzausbreitung, bei welcher der Schmerz, anders als bei der Schmerzübertragung, innerhalb der Grenzen der betroffenen anatomischen Struktur bleibt. Zu Forschungszwecken können bei Be- darf spezifische Kriterien für Kiefergelenksschmerzen mit Schmerzübertragung gemäß der DC/TMD-Kriterien angewendet werden.

Es werden Unterformen mit einer systemischen oder nichtsystemischen Arthritis des Kiefergelenks mit aufgenommen, da Behandlungspläne und Prognosen anders ausfallen können je nachdem, ob die Arthritis lokalen oder systemischen Ursprungs ist.

Die Arbeitsgruppe erwog, einen Subtyp, ,idiopathische Kiefergelenksschmerzen" mit aufzunehmen. Da es jedoch hierbei zu einer erheblichen, wenn nicht sogar vollständigen Überschneidung mit 3.1 Primäre Kiefergelenksschmerzen käme, würde diese Diagnose wahrscheinlich keinen nützlichen Beitrag zur Forschung oder klinischen Arbeit leisten.

\subsection{Primäre Kiefergelenksschmerzen}

Beschreibung: Lokalisierter Schmerz im Kiefergelenk, der im Ruhezustand, bei Kieferbewegungen oder Palpation auftritt, ohne bekannte ursächliche Erkrankung. Die Diagnose entspricht vollständig der DC/TMD-Diagnose „TMJ Pain“ (Kiefergelenksschmerzen).

\section{Diagnostische Kriterien:}

A. Schmerzen in und/oder vor dem Ohr/den Ohren, die die Kriterien $B-D$ erfüllen

B. In einer oder mehreren Episoden ${ }^{1}$ auftretend und nicht remittierend

C. Beide der folgenden Punkte sind erfüllt:

1. Bei Untersuchung Bestätigung der Lokalisierung in der Region eines Kiefergelenks oder beider Kiefergelenke

2. Provoziert durch eines oder beides von Folgendem:

a) Palpation des lateralen Pols/der lateralen Pole des Kiefergelenkkopfs/der Kiefergelenkköpfe oder dessen/deren Umgebung

b) Maximale eigenständige oder unterstützte Kieferöffnung, rechts- oder linkslateral und/oder Protrusionsbewegung(en)

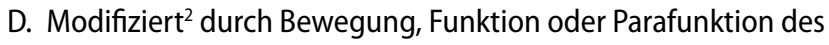
Kiefers (z. B. Zähneknirschen oder Zusammenbeißen der Zähne)

E. Nicht besser erklärt durch eine andere ICOP-Diagnose

\section{Anmerkungen:}

1. Schmerzepisoden können innerhalb eines Tages einmalig oder wiederholt auftreten, wobei eine Episode jeweils mindestens 30 Minuten andauert und die Gesamtdauer mindestens 2 Stunden am Tag beträgt.

2. Der Schmerz kann zu- oder abnehmen.

\subsubsection{Akute primäre Kiefergelenksschmerzen}

Beschreibung: Primäre Schmerzen im Kiefergelenk mit Schmerzbeginn innerhalb der letzten 3 Monate.

\section{Diagnostische Kriterien:}
A. Schmerzen, die die Kriterien für 3.1 Primäre Kiefergelenks- schmerzen sowie Kriterium B erfüllen
B. Schmerzbeginn innerhalb der letzten 3 Monate 
3.1.2 Chronische primäre Kiefergelenksschmerzen

Beschreibung: Primäre Schmerzen im Kiefergelenk mit Beginn vor mehr als 3 Monaten.

\section{Diagnostische Kriterien:}
A. Schmerzen, die die Kriterien für 3.1 Primäre Kiefergelenks- schmerzen sowie die Kriterien $B$ und $C$ erfüllen
B. Schmerzbeginn vor $>3$ Monaten
C. In mindestens 10 Episoden rezidivierend oder Dauerschmerz

\subsubsection{Chronische primäre gelegentliche Kiefergelenksschmerzen} Diagnostische Kriterien:

A. Schmerzen, die die Kriterien für 3.1.2 Chronische primäre Kiefergelenksschmerzen sowie Kriterium $B$ erfüllen

B. An $<1$ Tag/Monat auftretend

\subsubsection{Chronische häufige primäre Kiefergelenksschmerzen Diagnostische Kriterien:}

A. Schmerzen, die die Kriterien für 3.1.2 Chronische primäre Kiefergelenksschmerzen sowie Kriterium B erfüllen

B. Durchschnittlich für $>3$ Monate an 1-14 Tagen/Monat auftretend ( $>12$ und $<180$ Tage/Jahr)

3.1.2.2.1 Chronische häufige primäre Kiefergelenksschmerzen ohne Schmerzübertragung

Diagnostische Kriterien:

A. Schmerzen, die die Kriterien für 3.1.2.2 Chronische häufige primäre Kiefergelenksschmerzen sowie Kriterium $B$ erfüllen

B. Schmerz bei Palpation des Kiefergelenks, der am unmittelbaren Ort der Tastuntersuchung lokalisiert ist

3.1.2.2.2 Chronische häufige primäre Kiefergelenksschmerzen mit Schmerzübertragung

Diagnostische Kriterien:

A. Schmerzen, die die Kriterien für 3.1.2.2 Chronische häufige primäre Kiefergelenksschmerzen sowie Kriterium B erfüllen

B. Schmerz bei Palpation des Kiefergelenks, der über die Gelenkregion hinausgeht

\subsubsection{Chronische sehr häufige primäre Kiefergelenksschmerzen}

\section{Diagnostische Kriterien:}

A. Schmerzen, die die Kriterien für 3.1.2 Chronische primäre Kiefergelenksschmerzen sowie Kriterium B erfüllen

B. Durchschnittlich für $>3$ Monate an $>15$ Tagen/Monat $(>180$ Tage/Jahr) auftretend

3.1.2.3.1 Chronische sehr häufige primäre Kiefergelenksschmerzen ohne Schmerzübertragung

\section{Diagnostische Kriterien:}

A. Schmerzen, die die Kriterien für 3.1.2.3 Chronische sehr häufige primäre Kiefergelenksschmerzen sowie Kriterium B erfüllen

B. Schmerz bei Palpation des Kiefergelenks, der am unmittelbaren Ort der Tastuntersuchung lokalisiert ist
3.1.2.3.2 Chronische sehr häufige primäre Kiefergelenksschmerzen mit Schmerzübertragung

\section{Diagnostische Kriterien:}

A. Schmerzen, die die Kriterien für 3.1.2.3 Chronische sehr häufige primäre Kiefergelenksschmerzen sowie Kriterium B erfüllen

B. Schmerz bei Palpation des Kiefergelenks, der über die Gelenkregion hinausgeht

\subsection{Sekundäre Kiefergelenksschmerzen}

Beschreibung: Schmerzen, die im Kiefergelenk lokalisiert sind und für die eine andere identifizierte Erkrankung ursächlich ist, etwa eine Entzündung (zum Beispiel aufgrund eines Traumas, einer Infektion, Kristallablagerung oder Autoimmunerkrankung), eine Sensibilisierung des Gewebes, strukturelle Veränderungen (wie etwa eine Osteoarthrose, Diskusverlagerung oder Subluxation) oder eine Verletzung.

Diagnostische Kriterien:

A. Schmerzen im Kiefer, den Schläfen/der Schläfe, im Ohr/in den Ohren und/oder vor dem Ohr/den Ohren, die die Kriterien C und $D$ erfüllen

B. Es wurde eine Grunderkrankung diagnostiziert ${ }^{2}$, die bekanntermaßen Kiefergelenksschmerzen ${ }^{1}$ auslösen kann

C. Der Schmerz weist alle der folgenden drei Charakteristika auf: 1. In der Region des Kiefergelenks/der Kiefergelenke lokalisiert, durch Untersuchung bestätigt

2. Provoziert durch eines oder beides von Folgendem:

a) Palpation des lateralen Pols/der lateralen Pole des Kiefergelenkkopfs/der Kiefergelenkköpfe oder dessen/deren Umgebung

b) Maximale eigenständige oder unterstützte Kieferöffnung, rechts- oder linkslateral und/oder Protrusionsbewegung(en)

3. Modifiziert ${ }^{4}$ durch Bewegung, Funktion oder Parafunktion des Kiefers (z. B. Zähneknirschen oder Zusammenbeißen der Zähne)

D. Der Nachweis des ursächlichen Zusammenhangs besteht aufgrund von mindestens zwei der folgenden Punkte: 5

1. Der Schmerz hat sich in einem zeitlichen Zusammenhang mit dem Beginn oder der erheblichen Verschlimmerung der vermuteten ursächlichen Erkrankung entwickelt oder hat zu deren Entdeckung geführt

2. Der Schmerz hat sich parallel zur Progression der angenommenen ursächlichen Erkrankung deutlich ${ }^{6}$ verschlimmert

3. Der Schmerz hat sich deutlich ${ }^{6}$ gebessert oder gelegt parallel zur Besserung oder zu dem Verschwinden ${ }^{7}$ der angenommenen ursächlichen Erkrankung

E. Nicht besser erklärt durch eine andere ICOP-Diagnose

\section{Anmerkungen:}

1. Die Erkrankung wird bei jeder Unterform spezifiziert.

2. Die Diagnose erfolgt gemäß der Definition der Expanded DC/TMD Taxonomy.

3. Diese Zeichen lassen sich bei der körperlichen Untersuchung demonstrieren oder werden, bei Schmerzen die sich bereits wieder gelegt haben, anamnestisch berichtet. 
4. Der Schmerz kann zu- oder abnehmen.

5. Zusätzliche und/oder alternative Nachweise für ursächliche Zusammenhänge werden bei einigen Unterformen spezifiziert.

6. Der Patient beschreibt eine deutliche Veränderung der Intensität.

7. Spontan oder durch Behandlung.

\subsubsection{Kiefergelenksschmerzen zurückzuführen auf eine Arthritis}

Beschreibung: Kiefergelenksschmerzen, die durch eine persistierende Entzündung von Gelenkgewebe ausgelöst werden (zum Beispiel aufgrund eines Traumas, einer Infektion, Kristallablagerung oder Autoimmunerkrankung). Kiefergelenksschmerzen sind bei einer Kiefergelenkarthritis weit verbreitet, doch kann auch eine schmerzlose Kiefergelenkarthritis vorliegen.

\section{Diagnostische Kriterien:}

A. Schmerzen, die die Kriterien für 3.2 Sekundäre Kiefergelenksschmerzen sowie Kriterium C erfüllen

B. Es wurde eine Arthritis des Kiefergelenks diagnostiziert

C. Der Nachweis eines ursächlichen Zusammenhangs zeigt sich in einem oder beiden der folgenden Punkte:

1. Der Schmerz hat sich in engem zeitlichem Zusammenhang mit der Arthritis des Kiefergelenks entwickelt oder führte zu ihrer Diagnose

2. Eines oder beides von Folgendem trifft zu:

a) Der Schmerz hat sich parallel zu der Verschlimmerung der Arthritis des Kiefergelenks deutlich' verschlimmert

b) Der Schmerz hat sich mit der Behandlung der Arthritis des Kiefergelenks deutlich ${ }^{1}$ gebessert oder aufgelöst

D. Nicht besser erklärt durch eine andere ICOP-Diagnose

\section{Anmerkung:}

1. Dergestalt, dass der Patient eine deutliche Veränderung der Intensität beschreibt.

\subsubsection{Kiefergelenksschmerzen zurückzuführen auf eine nichtsyste-} mische Arthritis

\section{Diagnostische Kriterien:}

A. Schmerzen, die die Kriterien für 3.2.1 Kiefergelenksschmerzen zurückzuführen auf eine Arthritis erfüllen

B. Eines von Folgendem trifft zu:

1. Es findet sich kein Nachweis einer rheumatischen Erkrankung

2. Es besteht der Nachweis einer systemischen entzündlichen Gelenkerkrankung, aber deren Verbindung mit den Kiefergelenksschmerzen ist nicht belegt

\subsubsection{Kiefergelenksschmerzen zurückzuführen auf eine systemische} Arthritis

\section{Diagnostische Kriterien:}

A. Schmerzen, die die Kriterien für 3.2.1 Kiefergelenksschmerzen zurückzuführen auf eine Arthritis sowie Kriterium C erfüllen

B. Es besteht der Nachweis einer systemischen entzündlichen Gelenkerkrankung

C. Beide der folgenden Punkte sind erfüllt:
1. Der Schmerz hat sich in einem engen zeitlichen Zusammenhang mit anderen Symptomen und/oder klinischen oder biologischen Anzeichen für den Beginn der systemischen entzündlichen Gelenkerkrankung entwickelt oder hat zu deren Diagnose geführt

2. Eines oder beides von Folgendem trifft zu:

a) Der Schmerz hat sich parallel zu der Verschlechterung der systemischen entzündlichen Gelenkerkrankung deutlich ${ }^{1}$ verstärkt

b) Der Schmerz hat sich mit der Behandlung der systemischen entzündlichen Gelenkerkrankung deutlich ${ }^{1}$ gebessert oder aufgelöst

\section{Anmerkung:}

1. Dergestalt, dass der Patient eine deutliche Veränderung der Intensität beschreibt.

\subsubsection{Kiefergelenksschmerzen zurückzuführen auf eine Diskusverlagerung}

Beschreibung: Kiefergelenksschmerzen verursacht durch eine Diskusverlagerung des Kiefergelenks bei Fehlen einer Arthritis des Kiefergelenks. Hier können Kiefergelenksschmerzen auf mechanische Schädigungen im Gelenk zurückgehen.

Kommentar: Derzeit existieren keine konkreten Kriterien, nach denen sich eine Verbindung zwischen Kiefergelenksschmerzen und einer Diskusverlagerung mit oder ohne Reduktion herstellen lässt. Es besteht jedoch Grund zu der Annahme, dass eine Diskusverlagerung unter bestimmten Umständen beim Kieferschluss Kiefergelenksschmerzen auslösen kann, was impliziert, dass die Kiefergelenksschmerzen sekundärer Natur sind. Dieses Thema bedarf noch weiterer Forschung, um optimale Kriterien zu entwickeln. Ein möglicher Ausgangspunkt wären die hier gemachten Vorschläge.

\subsubsection{Kiefergelenksschmerzen zurückzuführen auf eine Diskusver-} lagerung mit Reduktion

\section{Diagnostische Kriterien:}

A. Schmerzen, die die Kriterien für 3.2 Sekundäre Kiefergelenksschmerzen sowie Kriterium C erfüllen

B. Es wurde anhand beider folgenden Punkte eine Diskusverlagerung des Kiefergelenks mit Reduktion diagnostiziert:

1. Bericht über (ein) Geräusch(e) im Kiefergelenk bei Bewegung oder Ausübung von Funktionen des Kiefers in den letzten 30 Tagen und/oder bei der Untersuchung

2. Bei der Palpation mindestens ein Mal aus drei Wiederholungen wahrgenommene Klick-, Knack- und/oder Schnappgeräusch(e) entweder:

a) Beim Öffnen und Schließen der Kiefer

b) Bei:

i. Öffnen oder Schließen der Kiefer

ii. Rechts- oder linkslateralen und/oder Protrusionsbewegungen

C. Der Nachweis des ursächlichen Zusammenhangs besteht aufgrund von mindestens zwei der folgenden Punkte:

1. Der Schmerz fällt exakt mit dem/den Klick-, Knack- und/ oder Schnappgeräusch(en) zusammen 
2. Der Schmerz hat sich in engem zeitlichen Zusammenhang mit der Diskusverlagerung entwickelt oder hat zu ihrer Diagnose geführt

3. Eines oder beides von Folgendem trifft zu:

a) Der Schmerz hat sich parallel zu der Zunahme der Diskusverlagerung deutlich ${ }^{1}$ verstärkt

b) Die Kiefergelenksschmerzen haben sich bei Behandlung der Diskusverlagerung deutlich ${ }^{1}$ gebessert oder aufgelöst

D. Nicht besser erklärt durch eine andere ICOP-Diagnose

\section{Anmerkung:}

1. Dergestalt, dass der Patient eine deutliche Veränderung der Intensität beschreibt.

3.2.2.1.1 Kiefergelenksschmerzen zurückzuführen auf eine Diskusverlagerung mit Reduktion, mit intermittierender Kieferklemme

\section{Diagnostische Kriterien:}

A. Kiefergelenksschmerzen, die die Kriterien für 3.2.2.1 Kiefergelenksschmerzen zurückzuführen auf eine Diskusverlagerung mit Reduktion erfüllen

B. In den letzten 30 Tagen ist eine intermittierende Kieferklem$\mathrm{me}^{1}$ bei begrenzter Mundöffnung aufgetreten, die sich in der Folge wieder löste

\section{Anmerkung:}

1. Und sei es nur vorübergehend.

\subsubsection{Kiefergelenksschmerzen zurückzuführen auf eine Diskusver-} lagerung ohne Reduktion

\section{Diagnostische Kriterien:}

A. Schmerzen, die die Kriterien für 3.2 Sekundäre Kiefergelenksschmerzen sowie Kriterium C erfüllen

B. Eine Diskusverlagerung des Kiefergelenks ohne Reduktion wurde anhand von beiden folgenden Punkten diagnostiziert:

1. Die Kieferbewegung ist gesperrt oder blockiert, was eine vollständige Öffnung verhindert

2. Eingeschränkte Öffnung des Kiefers, was beim Essen behindert

C. Der Nachweis eines ursächlichen Zusammenhangs ist durch einen oder mehrere der folgenden Punkte erbracht:

1. Der Schmerz hat sich in engem zeitlichen Zusammenhang mit der Diskusverlagerung entwickelt oder zu ihrer Diagnose geführt

2. Der Schmerz hat sich parallel zur Zunahme der Diskusverlagerung deutlich ${ }^{1}$ verstärkt

3. Der Schmerz hat sich mit der Behandlung der Diskusverlagerung deutlich ${ }^{1}$ verbessert oder aufgelöst

D. Nicht besser erklärt durch eine andere ICOP-Diagnose

\section{Anmerkung:}

1. Dergestalt, dass der Patient eine deutliche Veränderung der Intensität beschreibt.
3.2.3 Kiefergelenksschmerzen zurückzuführen auf eine degenerative Gelenkerkrankung

Beschreibung: Kiefergelenksschmerzen aufgrund einer degenerativen Gelenkerkrankung (Osteoarthrose, Osteoarthritis) bei Fehlen einer Arthritis des Kiefergelenks.

Kommentar: Die Definitionen der Begriffe "Osteoarthrose" und "Osteoarthritis" des Kiefergelenks sowie aller sonstiger Gelenke überlappen sich in der Fachliteratur. Ihre Handhabung ist uneinheitlich und unklar. So wurde zum Beispiel Osteoarthrose von einigen als eine primär nichtentzündliche Erkrankung des Gelenkknorpels betrachtet, mit einer hieraus resultierenden Veränderung des zugrundeliegenden Knochengewebes. In diesem Zusammenhang kann eine Osteoarthrose auch (oft) in Begleitung einer Arthritis auftreten. Bei anderen wurden diese Krankheitsbilder allesamt als Osteoarthritis bezeichnet. Historisch betrachtet unterschieden sich sogar Europa und die USA im Hinblick auf diese Definitionen. Ungeachtet dessen ist es wichtig zu berücksichtigen, dass Kiefergelenksschmerzen kein zuverlässiges Anzeichen für eine Arthritis sind. Das bedeutet, dass es viele Patienten ohne Kiefergelenksschmerzen gibt, die durchaus eine Arthritis (d. h. eine Entzündung in Geweben der Gelenke mit einer hieraus resultierenden Zerstörung von Knorpel- und Knochengewebe) aufweisen. Ein Beispiel zeigt sich bei der juvenilen idiopathischen Arthritis (JIA), bei der ungeachtet einer häufigen Beteiligung des Kiefergelenks nur selten von Kiefergelenksschmerzen berichtet wird. Andererseits gibt es Patienten mit einer Arthritis des Kiefergelenks, bei der Schmerzen das einzige Symptom darstellen und die ohne Zerstörung des Gewebes abläuft.

Stellt man sich ein vereinfachtes Modell des komplexen Entzündungsgeschehens vor, kann man sich die Entzündung als etwas vorstellen, das irgendwo auf einem Kontinuum von Schmerz ohne Zerstörung von Gewebe zum Gegenpol hierzu angesiedelt ist. Die vorgeschlagenen ICOP-Kriterien versuchen diese Faktoren zu berücksichtigen, um Forschung anzuregen, die hier Abklärung bringt und zu neuen Erkenntnissen beiträgt.

\section{Diagnostische Kriterien:}

A. Schmerzen im Kiefergelenk, die die Kriterien für 3.2 Sekundäre Kiefergelenksschmerzen sowie Kriterium C erfüllen

B. Es wurde angesichts der beiden folgenden Punkte eine degenerative Erkrankung des Kiefergelenks diagnostiziert:

1. Es wurde von einem Geräusch oder mehreren Geräuschen im Kiefergelenk bei Kieferbewegung oder -funktion in den letzten 30 Tagen berichtet und/oder derartiges tritt während der Untersuchung auf

2. Knirschendes Geräusch (Krepitus) bei Palpation bei maximaler eigenständiger oder unterstützter Öffnung sowie bei lateralen und/oder Protrusionsbewegungen

C. Der Nachweis eines ursächlichen Zusammenhangs wurde durch einen oder mehrere Punkte von Folgendem erbracht:

1. Der Schmerz hat sich in einem engen zeitlichen Zusammenhang mit der degenerativen Kiefergelenkserkrankung entwickelt oder zu deren Diagnose geführt

2. Der Schmerz hat sich parallel zu der Verstärkung der degenerativen Erkrankung des Kiefergelenks deutlich ${ }^{1}$ verstärkt 
3. Der Schmerz hat sich mit Behandlung der degenerativen Erkrankung des Kiefergelenks deutlich ${ }^{1}$ gebessert oder aufgelöst

D. Nicht besser erklärt durch eine andere ICOP-Diagnose

\section{Anmerkung:}

1. Dergestalt, dass der Patient eine deutliche Veränderung der Intensität beschreibt.

\subsubsection{Kiefergelenksschmerzen zurückzuführen auf eine Subluxation}

Beschreibung: Kiefergelenksschmerzen verursacht durch Subluxation bei Fehlen einer Arthritis des Kiefergelenks. In der Regel sind diese akut und dürften auf eine gewebliche Überdehnung zurückgehen.

\section{Diagnostische Kriterien:}

A. Schmerzen, die die Kriterien für 3.2 Sekundäre Kiefergelenksschmerzen sowie Kriterium C erfüllen

B. Es wurde eine Subluxation des Kiefergelenks durch beide folgenden Punkte diagnostiziert:

1. Kiefersperre oder -blockade ${ }^{1}$ in den letzten 30 Tagen, durch die eine Mundschließung aus der weit offenen Position verhindert wird

2. Unfähigkeit, den Mund ohne Durchführung eines speziellen manipulativen Manövers von einer offenen zu einer normalen geschlossenen Position zurückzubringen

C. Der Nachweis eines ursächlichen Zusammenhangs wurde durch einen oder mehrere der folgenden Punkte erbracht: 1. Der Schmerz hat sich in einem engen zeitlichen Zusammenhang mit der Subluxation entwickelt oder zu ihrer Diagnose geführt

2. Der Schmerz hat sich parallel zur Zunahme der Subluxation deutlich ${ }^{2}$ verstärkt

3. Der Schmerz hat sich mit Behandlung der Subluxation deutlich ${ }^{2}$ gebessert oder aufgelöst

D. Nicht besser erklärt durch eine andere ICOP-Diagnose

\section{Anmerkungen:}

1. Und sei es nur vorübergehend.

2. Dergestalt, dass der Patient eine deutliche Veränderung der Intensität beschreibt.

\section{Literatur}

Alstergren P, Pigg M und Kopp S. Clinical diagnosis of temporomandibular joint arthritis. J Oral Rehabil 2018; 45: 269-281.

Masuda M, lida T, Exposto FG, et al. Referred pain and sensations evoked by standardized palpation of the masseter muscle in healthy participants. J Oral Facial Pain Headache 2018; 32: 159-166.

Schiffman E, Ohrbach R, Truelove E, et al. Diagnostic Criteria for Temporomandibular Disorders (DC/TMD) for clinical and research applications: recommendations of the International RDC/TMD Consortium Network and Orofacial Pain Specia Interest Group. J Oral Facial Pain Headache 2014; 28: 6-27.

Svensson P, Michelotti A, Lobbezoo F, et al. The many faces of persistent orofacial muscle pain. J Oral Facial Pain Headache 2015; 29: 207-208.

Treede RD, Rief W, Barke A, et al. A classification of chronic pain for ICD-11. Pain 2015; 156: 1003-1007.

\section{Orofaziale Schmerzen zurückzuführen auf eine Läsion oder Erkrankung der Hirnnerven}

An anderer Stelle klassifiziert: Die nachfolgenden, weniger häufigen klinischen Entitäten, bei denen Schmerzen primär außerhalb der orofazialen Region auftreten, werden in der ICHD-3 und nicht in der ICOP klassifiziert:

13.3 Schmerz zurückzuführen auf eine Läsion oder Erkrankung des N. intermedius, 13.4 Okzipitalisneuralgie, 13.5 Nacken-ZungenSyndrom (engl. Neck-tongue syndrome), 13.6 Schmerzhafte Optikusneuritis, 13.7 Kopfschmerz zurückzuführen auf eine ischämische Lähmung des N. oculumotorius, 13.8 Tolosa-Hunt-Syndrom, 13.9 Paratrigeminales okulosympathisches (Raeder-) Syndrom, 13.10 Rezidivierende schmerzhafte ophthalmoplegische Neuropathie, 13.13 Zentraler neuropathischer Schmerz.

Allgemeine Kommentare: Dieser Abschnitt basiert in weiten Teilen auf der ICHD-3 und der 11. Version der Internationalen Klassifikation der Krankheiten (International Classification of Diseases 11th Revision, ICD-11)/International Association for the Study of Pain (IASP), wobei einige kleine Änderungen vorgenommen wurden. Idiopathische Erkrankungen (d. h. anhaltender idiopathischer Gesichtsschmerz und Syndrom des brennenden Mundes (engl. Burning mouth syndrome, BMS) wurden unter 6. Idiopathische orofaziale Schmerzen eingeordnet, da noch keine ausreichenden Belege dafür vorliegen, dass es sich hierbei um zweifelsfrei neuropathische Schmerzen handelt. Bei bestimmten Störungsbildern haben wir dem Begriff neuropathische Schmerzen gegenüber schmerzhafter Neuropathie den Vorzug gegeben, um uns an die IASP/ICD-11-Kriterien anzugleichen; während die ICHD-3 den letztgenannten Begriff unterstützt, schwenkt die Fachliteratur zum Thema Schmerz sowie die IASP/ICD-11 mittlerweile häufig zum erstgenannten Terminus über.

\subsection{Schmerzen zurückzuführen auf eine Läsion oder Erkrankung des N. trigeminus}

\subsubsection{Trigeminusneuralgie \\ Früher verwendeter Begriff: Tic douloureux.}

Beschreibung: Die Trigeminusneuralgie ist ein wiederkehrender einseitiger Gesichtsschmerz, der durch kurze, stromstoßartige, plötzlich beginnende und endende Schmerzattacken gekennzeichnet ist, die auf das Versorgungsgebiet eines einzelnen Astes oder mehrerer Äste des N. trigeminus beschränkt sind. Der Schmerz wird gewöhnlich durch harmlose Reize ausgelöst. Er kann sich ohne offenkundige Ursache entwickeln oder Folge einer anderen Störung sein. Zusätzlich kann er in Begleitung eines Dauerschmerzes von mittelstarker Intensität im/in den Versorgungsbereich(en) des betroffenen Nervenastes/der betroffenen Nervenäste auftreten.

\section{Diagnostische Kriterien:}

A. Wiederkehrende paroxysmale unilaterale Gesichtsschmerzattacken im/in den Versorgungsbereich(en), die einen Ast oder mehrere Äste des N. trigeminus betreffen, ohne Ausstrahlung darüberhinaus ${ }^{1}$, die die Kriterien $B$ und $C$ erfüllen 
B. Der Schmerz weist alle der folgenden Charakteristika auf:

1. Dauer zwischen einem Sekundenbruchteil und 2 Minuten $^{2}$

2. Starke Intensität ${ }^{3}$

3. Stromstoßartige, einschießende, stechende oder scharfe Qualität

C. Vorangegangene harmlose Reize im betroffenen Versorgungsbereich des $\mathrm{N}$. trigeminus ${ }^{4}$

D. Nicht besser erklärt durch eine andere ICOP oder ICHD-3-Diagnose

\section{Anmerkungen:}

1. Bei einer kleinen Zahl von Patienten kann der Schmerz zu einem anderen Ast ausstrahlen, bleibt jedoch innerhalb der trigeminalen Dermatome.

2. Die Schmerzdauer kann sich im Laufe der Zeit dahingehend ändern, dass die Paroxysmen länger anhalten. Eine Minderheit von Patienten berichtet von Attacken, die vorwiegend $>2 \mathrm{Mi}-$ nuten anhalten.

3. Die Schmerzintensität kann im Laufe der Zeit zunehmen.

4. Einige Attacken können spontan auftreten oder spontan wirken, doch muss in der Vorgeschichte ein von harmlosen Reizen ausgelöster Schmerz aufgetreten sein, damit dieses Kriterium erfüllt ist. Im Idealfall sollte der untersuchende Kliniker die anamnestischen Angaben durch Wiederholung des auslösenden Phänomens zu bestätigen suchen. Dies mag jedoch aufgrund einer entsprechenden Weigerung des Patienten bzw. einer anatomisch ungünstigen Lokalisation des Triggers und/oder anderer Faktoren nicht immer möglich sein.

Kommentar: Unabhängig von dem auslösenden Phänomen sind bei den meisten Patienten mit einer 4.1.1 Trigeminusneuralgie keine sensorischen Anomalien im Versorgungsbereich des N. trigeminus nachweisbar, es sei denn mit Hilfe modernster Verfahren (z. B. einer Quantitativen Sensorischen Testung). Bei einigen Patienten kann die klinische neurologische Untersuchung jedoch sensible Defizite ergeben, die Anlass zu bildgebenden Untersuchungen sein sollten, um die mögliche Ursache zu erkunden. Eine Diagnose von Unterformen wie 4.1.1.1 Klassische Trigeminusneuralgie, 4.1.1.2 Sekundäre Trigeminusneuralgie oder 4.1.1.3 Idiopathische Trigeminusneuralgie wird so ermöglicht.

Bei starker Schmerzintensität löst der Schmerz häufig eine Kontraktion der Gesichtsmuskeln auf der betroffenen Seite aus (Tic douloureux).

Es können leichte autonome Symptome wie ein Tränen und/ oder eine Rötung des ipsilateralen Auges vorliegen.

Einem schmerzhaften Paroxysmus folgt gewöhnlich eine refraktäre Phase, in der keine Schmerzen ausgelöst werden können.

\subsubsection{Klassische Trigeminusneuralgie}

Früher verwendeter Begriff: Primäre Trigeminusneuralgie.

Beschreibung: Eine Trigeminusneuralgie, die sich ohne Beteiligung einer anderen offensichtlichen Ursache als der einer neurovaskulären Kompression entwickelt.

\section{Diagnostische Kriterien:}

A. Wiederkehrende paroxysmale einseitige Gesichtsschmerzattacken, die die Kriterien für eine 4.1.1 Trigeminusneuralgie erfüllen

B. Nachweis einer neurovaskulären Kompression (nicht nur Kontakt) mittels MRT oder während eines chirurgischen Eingriffs, mit morphologischen Veränderungen ${ }^{1}$ in der Wurzel des N. trigeminus

\section{Anmerkung:}

1. Üblicherweise Atrophie oder Verdrängung.

Kommentar: Eine Atrophie und/oder Verdrängung der Trigeminuswurzel infolge einer neurovaskulären Kompression tritt unabhängig in Begleitung der Zeichen und Symptome einer 4.1.1 Trigeminusneuralgie auf. Bei Vorliegen dieser anatomischen Veränderung wird die Erkrankung als 4.1.1.1 Klassische Trigeminusneuralgie diagnostiziert.

Üblicher Situs der neurovaskulären Kompression ist die Eintrittszone der Trigeminuswurzel, wobei eine Kompression durch eine Arterie eindeutiger von Symptomen begleitet ist als die Kompression durch eine Vene. Es stehen MRT-Verfahren zur Messung des Volumens und der Querschnittsfläche der Wurzel zur Verfügung. Zu den atrophischen Veränderungen können Demyelinisierung, ein neuronaler Zellverlust, Veränderungen der Mikrogefäße und andere morphologische Veränderungen gehören. Die genauen Mechanismen, wie atrophische Veränderungen des N. trigeminus zur Schmerzentstehung beitragen, sind zwar ungeklärt, manches deutet jedoch darauf hin, dass diese, wenn sie vor der Operation vorliegen, nach einer mikrovaskulären Dekompression ein positives Ergebnis erwarten lassen.

4.1.1.1.1 Klassische Trigeminusneuralgie, rein paroxysmal Beschreibung: Klassische Trigeminusneuralgie ohne anhaltenden Hintergrundschmerz.

\section{Diagnostische Kriterien:}

A. Wiederkehrende paroxysmale einseitige Gesichtsschmerzattacken, die die Kriterien für eine 4.1.1.1 Klassische Trigeminusneuralgie erfüllen

B. Schmerzfreiheit im betroffenen Versorgungsbereich des N. trigeminus zwischen den Attacken

Kommentar: Die 4.1.1.1.1 Klassische Trigeminusneuralgie, rein paroxysmal spricht üblicherweise, zumindest initial, auf eine Pharmakotherapie an (insbesondere auf Gaben von Carbamazepin oder Oxcarbazepin).

4.1.1.1.2 Klassische Trigeminusneuralgie mit Dauerschmerz Früher verwendeter Begriffe: Atypische Trigeminusneuralgie; Trigeminusneuralgie Typ 2.

Beschreibung: Klassische Trigeminusneuralgie mit anhaltendem Hintergrundschmerz. 


\section{Diagnostische Kriterien:}

A. Wiederkehrende paroxysmale einseitige Gesichtsschmerzattacken, die die Kriterien für eine 4.1.1.1 Klassische Trigeminusneuralgie erfüllen

B. Begleitende durchgehende oder fast durchgehende Schmerzen im betroffenen Versorgungsgebiet des N. trigeminus zwischen den Attacken

Kommentar: Der Dauerschmerz kann auf eine periphere oder zentrale Sensibilisierung zurückgehen.

\subsubsection{Sekundäre Trigeminusneuralgie}

Beschreibung: Trigeminusneuralgie verursacht durch eine Grunderkrankung. Die klinische Untersuchung ergibt bei einem signifikanten Prozentsatz dieser Patienten sensible Veränderungen.

\section{Diagnostische Kriterien:}

A. Wiederkehrende paroxysmale einseitige Gesichtsschmerzattacken, die die Kriterien für eine 4.1.1 Trigeminusneuralgie erfüllen, sei es rein paroxysmal oder in Begleitung von durchgehenden oder beinahe durchgehenden Schmerzen

B. Es wurde eine Grunderkrankung nachgewiesen ${ }^{1}$, die bekanntermaßen die Neuralgie verursachen kann und erklärt ${ }^{2}$

C. Nicht besser erklärt durch eine andere ICOP- oder ICHD-3-Diagnose

\section{Anmerkungen:}

1. Am besten eignet sich eine MRT, um die zugrunde liegende Ursache für eine 4.1.1.2 Sekundäre Trigeminusneuralgie zu entdecken. Weitere Untersuchungen können eine neurophysiologische Messung von Trigeminusreflexen und trigeminal evozierten Potentialen umfassen, die für Patienten geeignet sind, die keiner MRT unterzogen werden können.

2. Anerkannte Ursachen sind ein Tumor im Kleinhirnbrückenwinkel, eine AV-Malformation und multiple Sklerose.

4.1.1.2.1 Trigeminusneuralgie zurückzuführen auf multiple Sklerose

Beschreibung: Trigeminusneuralgie verursacht durch MultipleSklerose(MS)-Plaque oder -Plaques in der Pons oder der Eintrittszone der Trigeminuswurzel und verbunden mit anderen Symptomen und/oder klinischen Zeichen oder Laborbefunden, die auf MS schließen lassen.

\section{Diagnostische Kriterien:}

A. Wiederkehrende paroxysmale einseitige Gesichtsschmerzattacken, die die Kriterien für eine 4.1.1 Trigeminusneuralgie erfüllen

B. Beide der folgenden Punkte sind erfült:

1. Es wurde eine multiple Sklerose (MS) diagnostiziert

2. Es wurde im MRT eine MS-Plaque an der Eintrittszone der Trigeminuswurzel oder in der Pons diagnostiziert, die Auswirkungen auf die intrapontinen primär-afferenten Fasern hat bzw. auf deren Vorliegen elektrophysiologische Routineuntersuchungen' schließen lassen, die eine Beeinträchtigung der trigeminalen Leitungsbahnen zeigen
C. Nicht besser erklärt durch eine andere ICOP oder ICHD-3-Diagnose

\section{Anmerkung:}

1. Blinkreflex oder trigeminal evozierte Potenziale.

Kommentar: Eine 4.1.1.2.1 Trigeminusneuralgie zurückzuführen auf multiple Sklerose tritt bei 2 bis $5 \%$ der Patienten mit multipler Sklerose (MS) auf, mitunter beidseits. Umgekehrt wird bei nur 2 bis $4 \%$ der Fälle mit 4.1.1 Trigeminusneuralgie eine multiple Sklerose entdeckt. Symptome einer Trigeminusneuralgie sind selten ein Leitmerkmal von MS.

Die Läsion in der Pons wirkt sich auf die intrapontinen zentralen Endigungen der trigeminalen afferenten Fasern aus, die zu den trigeminalen Hirnstammkernen projizieren. Pontine Läsionen, die sich auf die Neuronen zweiter Ordnung des trigeminothalamischen Traktes auswirken, führen üblicherweise zu nichtparoxysmalen Schmerzattacken und/oder Dysästhesien und sollten unter der ICDH-3-Diagnose 13.13.1 ZentralerneuropathischerSchmerzzurückzuführen auf multiple Sklerose klassifiziert werden.

Bei einigen Patienten mit MS findet sich eine neurovaskuläre Kompression der Trigeminuswurzel. Man geht davon aus, dass MS die Anfälligkeit der Nervenwurzel für die Auswirkungen einer Kompression erhöht und so eher zu paroxysmalen Schmerzattacken führt.

Patienten mit einer 4.1.1.2.1 Trigeminusneuralgie zurückzuführen auf multiple Sklerose profitieren weniger von pharmakologischen und chirurgischen Interventionen als solche mit einer 4.1.1.1 Klassischen Trigeminusneuralgie.

4.1.1.2.2 Trigeminusneuralgie zurückzuführen auf eine raumfordernde Läsion

Beschreibung: Trigeminusneuralgie verursacht durch einen Kontakt zwischen dem betroffenen Trigeminusnerv und einer raumfordernden Läsion.

\section{Diagnostische Kriterien:}

A. Wiederkehrende paroxysmale einseitige Gesichtsschmerzattacken die die Kriterien für 4.1.1 Trigeminusneuralgie erfüllen

B. Beide der folgenden Punkte sind erfüllt:

1. Es wurde mit einem bildgebenden Verfahren eine raumfordernde Läsion nachgewiesen, die Kontakt mit dem betroffenen Trigeminusnerven hat

2. Die Schmerzen haben sich nach Identifikation der Läsion entwickelt oder führten zu ihrer Entdeckung

C. Nicht besser erklärt durch eine andere ICOP oder ICHD-3-Diagnose

Kommentar: Patienten mit einer 4.1.1.2.2 Trigeminusneuralgie zurückzuführen auf eine raumfordernde Läsion können klinisch nachweisbare sensible Zeichen aufweisen oder nicht, wohingegen elektrophysiologische Tests wie die Prüfung der trigeminalen Hirnstammreflexe in nahezu allen Fällen Auffälligkeiten ergeben. 
4.1.1.2.3 Trigeminusneuralgie zurückzuführen auf eine andere Ursache

Beschreibung: Trigeminusneuralgie, die durch eine andere Grunderkrankung als die oben beschriebenen verursacht wird.

\section{Diagnostische Kriterien:}

A. Wiederkehrende einseitige paroxysmale Gesichtsschmerzattacken, die die Kriterien für eine 4.1.1 Trigeminusneuralgie erfüllen, entweder rein paroxysmal oder in Begleitung gleichzeitiger Dauer- oder annähernder Dauerschmerzen, doch nicht unbedingt einseitig

B. Beide der folgenden Punkte sind erfüllt:

1. Es wurde eine andere Erkrankung als die oben beschriebenen diagnostiziert, die jedoch bekanntermaßen eine Trigeminusneuralgie ${ }^{1}$ auslösen kann

2. Der Schmerz hat sich nach dem Beginn der Erkrankung entwickelt oder führte zu ihrer Entdeckung

C. Nicht besser erklärt durch eine andere ICOP oder ICHD-3-Diagnose

\section{Anmerkung:}

1. Anerkannte Ursachen sind eine Fehlbildung des Schädelbasisknochens, eine Bindegewebserkrankung, eine arteriovenöse Malformation, eine durale arteriovenöse Fistel sowie genetische Ursachen einer Neuropathie oder neuronalen Übererregbarkeit.

\subsubsection{Idiopathische Trigeminusneuralgie}

Beschreibung: Trigeminusneuralgie, bei der weder elektrophysiologische Tests noch das MRT signifikante Auffälligkeiten erkennen lassen.

\section{Diagnostische Kriterien:}

A. Wiederkehrende paroxysmale einseitige Gesichtsschmerzattacken, die die Kriterien für eine 4.1.1 Trigeminusneuralgie erfüllen, entweder rein paroxysmal oder in Begleitung gleichzeitiger Dauer- oder annähernder Dauerschmerzen

B. Weder eine 4.1.1.1 Klassische Trigeminusneuralgie noch eine 4.1.1.2 Sekundäre Trigeminusneuralgie wurde durch entsprechende Untersuchungen ${ }^{1,2}$ bestätigt

C. Nicht besser erklärt durch eine andere ICOP- oder ICHD-3-Diagnose

\section{Anmerkungen:}

1. Einschließlich elektrophysiologischer Tests oder MRT.

2. Ein Kontakt zwischen einem Blutgefäß und dem Trigeminusnerv und/oder der Wurzel des Trigeminusnervs ist bei bildgebenden Untersuchungen an gesunden Probanden ein gängiger Befund. Zeigt sich ein solcher Kontakt bei Vorliegen einer 4.1.1 Trigeminusneuralgie, doch ohne Belege für morphologische Veränderungen (d.h., Atrophie oder Verdrängung) in der Nervenwurzel, so sind die Kriterien für eine 4.1.1.1 Klassische Trigeminusneuralgie nicht erfüllt und die Erkrankung wird als idiopathisch betrachtet.
4.1.1.3.1 Idiopathische Trigeminusneuralgie, rein paroxysmal Diagnostische Kriterien:

A. Wiederkehrende paroxysmale einseitige Gesichtsschmerzattacken, die die Kriterien für eine 4.1.1.3 Idiopathische Trigeminusneuralgie erfüllen

B. Es besteht zwischen den Attacken Beschwerdefreiheit im betroffenen Versorgungsbereich des N. trigeminus

4.1.1.3.2 Idiopathische Trigeminusneuralgie mit Dauerschmerz Diagnostische Kriterien:

A. Wiederkehrende paroxysmale einseitige Gesichtsschmerzattacken, die die Kriterien für eine 4.1.1.3 Idiopathische Trigeminusneuralgie erfüllen

B. Begleitender Dauerschmerz oder annähernder Dauerschmerz zwischen den Attacken im betroffenen Versorgungsbereich des N. trigeminus

\subsubsection{Andere neuropathische Schmerzen im Innervationsgebiet des N. trigeminus}

Beschreibung: Gesichtsschmerz im/in den Versorgungsbereich/ en eines einzelnen Astes oder mehrerer Äste des N. trigeminus, der durch eine andere Erkrankung verursacht wird und auf eine neuronale Schädigung hinweist. Der primäre Schmerz ist üblicherweise ein Dauerschmerz oder annähernder Dauerschmerz und wird in der Regel als brennend, drückend oder nadelstichartig beschrieben. Überlagerte kurze paroxysmale Schmerzattacken können auftreten, doch sind diese nicht der vorherrschende Schmerztyp. Diese Kombination unterscheidet die schmerzhafte Trigeminusneuropathie von der Trigeminusneuralgie. Es finden sich klinisch nachweisbare somatosensorische Veränderungen innerhalb des Versorgungsbereichs des Trigeminusnervs, und mechanische Allodynie und Kältehyperalgesie/-allodynie erfüllen die IASP-Kriterien für neuropathische Schmerzen. Im Regelfall sind allodynische Areale viel größer als die punktförmigen Triggerzonen, die bei einer 4.1.1 Trigeminusneuralgie vorliegen.

\subsubsection{Schmerzhafte Trigeminusneuropathie zurückzuführen auf Herpes Zoster}

Beschreibung: Einseitiger Gesichtsschmerz von weniger als 3 Monaten Dauer im/in den Verteilungsgebiet/en eines Astes oder mehrerer Äste des N. trigeminus, verursacht durch und begleitet von anderen Symptomen und/oder klinischen Zeichen eines akuten Herpes Zoster.

\section{Diagnostische Kriterien:}

A. Einseitiger Gesichtsschmerz im/in den Versorgungsbereich(en) eines Astes oder von Ästen des N. trigeminus mit einer Dauer von $<3$ Monaten

B. Einer oder mehrere der folgenden Punkte treffen zu:

1. Eine Herpeseruption ist im gleichen Versorgungsbereich des N. trigeminus wie der Schmerz aufgetreten

2. Mittels Polymerase-Kettenreaktion (PCR) wurde ein Varicella-Zoster-Virus (VZV) im Liquor nachgewiesen

3. Der direkte Immunfluoreszenztest auf VZV-Antigen oder PCR-Test auf VZV-DNA in vom Boden der Läsionen gewonnenen Zellen ist positiv 
C. Nicht besser erklärt durch eine andere ICOP- oder ICHD-3-Diagnose

Kommentar: Herpes Zoster zeigt in 10-15\% der Fälle Auswirkungen auf das Ganglion trigeminale, wobei bei rund $80 \%$ der Patienten der Augenast des N. trigeminus als betroffen ermittelt wurde.

Selten folgt dem Schmerz keine Eruption oder keine Effloreszenz (Zoster sine herpete). In solchen Fällen wird die Diagnose mittels einer Polymerase-Kettenreaktion gestellt, die eine Entdeckung von Varicella-Zoster-Virus-DNA im Liquor ermöglicht.

Eine 4.1.2.1 Schmerzhafte Trigeminusneuropathie zurückzuführen auf Herpes Zoster ist gewöhnlich brennend, stechend/einschießend, kribbelnd oder schmerzend und wird von einer kutanen Allodynie begleitet. Ophthalmischer Herpes kann in Begleitung einer Lähmung des 3., 4. und/oder 6. Hirnnervs auftreten.

Herpes Zoster ist bei immungeschwächten Patienten häufig - er tritt bei etwa $10 \%$ der Patienten mit Lymphom und $25 \%$ der Patienten mit M. Hodgkin auf.

\subsubsection{Postherpetische Trigeminusneuralgie}

Früher verwendeter Begriff: Postherpetische Trigeminusneuropathie.

Beschreibung: Anhaltender oder mindestens 3 Monate lang auftretender einseitiger Gesichtsschmerz im/in den Versorgungsgebiet/en eines einzelnen Astes oder mehrerer Äste des N. trigeminus, mit variablen sensiblen Veränderungen, verursacht durch einen Herpes Zoster.

\section{Diagnostische Kriterien:}

A. Einseitiger Gesichtsschmerz im/in den Versorgungsgebiet/en eines Astes oder mehrerer Äste des N. trigeminus, der anhält oder für $>3$ Monate wiederkehrt und Kriterium C erfüllt

B. Der Herpes Zoster betrifft denselben Ast/dieselben Äste des N. trigeminus

C. Der Schmerz hat sich in einem zeitlichen Zusammenhang mit der Herpes-zoster-Infektion entwickelt ${ }^{1}$

D. Nicht besser erklärt durch eine andere ICOP- oder ICHD-3-Diagnose

\section{Anmerkung:}

1. Üblicherweise hat sich der Schmerz entwickelt, während die Effloreszenzen noch aktiv waren, gelegentlich jedoch auch später, nach Verheilen der Effloreszenzen. In solchen Fällen können als Folge der Herpeseruption blass- oder hellviolette Narben auftreten.

Kommentar: Ungeachtet ihres lange bevorzugten Namens, ist die postherpetische Neuralgie eigentlich eine Neuropathie oder Neuronopathie: Es wurden signifikante pathoanatomische Veränderungen im Nerv, im Ganglion und in der Nervenwurzel nachgewiesen. Bei einer 4.1.2.2 Postherpetischen Trigeminusneuralgie bestehen auch Belege für die Entzündung, die sich bis in den trigeminalen Hirnstammkomplex hinein ausdehnt.

Die postherpetische Neuralgie nach einem akuten Herpes Zoster tritt eher mit steigendem Lebensalter auf.
Der erste Ast des N. trigeminus ist bei einer 4.1.2.2 Postherpetischen Trigeminusneuralgie am häufigsten befallen, doch können auch der zweite und dritte Ast involviert sein.

Typischerweise ist der Schmerz bei der postherpetischen Neuralgie brennend und juckend, Letzteres mitunter sehr im Vordergrund stehend und außerordentlich störend. Zudem zeigen Patienten mit postherpetischer Neuralgie typischerweise ein eindeutiges sensibles Defizit sowie eine durch leichte Berührung evozierbare mechanische Allodynie im beteiligten Versorgungsgebiet des $\mathrm{N}$. trigeminus. Viele Patienten weisen jedoch nur geringe sensible Verluste auf und zeigen stattdessen erhöhte Reaktionen auf thermische und/oder punktuelle Reize.

\subsubsection{Posttraumatische schmerzhafte Trigeminusneuropathie}

Früher verwendete Begriffe: Anaesthesia dolorosa; schmerzhafte posttraumatische Trigeminusneuropathie.

Beschreibung: Ein- oder beidseitige Gesichtsschmerzen oder intraorale Schmerzen nach einem Trauma des N. trigeminus/der Nn. trigemini und hiervon verursacht, mit weiteren Symptomen und/ oder klinischen Zeichen einer funktionellen trigeminalen Störung sowie für mehr als 3 Monate anhaltend oder wiederkehrend.

\section{Diagnostische Kriterien:}

A. Schmerzen in einem neuroanatomisch plausiblen Areal innerhalb des Versorgungsgebiets/der Versorgungsgebiete eines Trigeminusnervs oder beider Trigeminusnerven, die für >3 Monate anhaltend oder wiederkehren und Kriterium $\mathrm{C}$ und D erfüllen

B. Beide der folgenden Punkte sind erfüllt:

1. Anamnestisch ermittelbare Schädigung des/der peripheren N. trigeminus/Nn. trigemini mechanischer, thermischer oder chemischer Art bzw. durch Bestrahlung

2. Diagnostischer Test ${ }^{1}$, der eine Läsion des/der peripheren $\mathrm{N}$. trigeminus/Nn. trigemini bestätigt, was die Schmerzen erklärt ${ }^{2}$

C. Beginn innerhalb von 6 Monaten nach dem traumatischen Ereignis $^{3}$

D. Begleitet von somatosensorischen Symptomen und/oder Zeichen in dem gleichen neuroanatomisch plausiblen Versorgungsgebiet

E. Nicht besser erklärt durch eine andere ICOP- oder ICHD-3-Diagnose

\section{Anmerkungen:}

1. Tests, die eine relevante, den N. trigeminus betreffende Läsion oder Erkrankung bestätigen, sind beispielsweise der chirurgische oder radiologische Nachweis einer Kompression oder Läsion des Nervs/der Nerven, eine Nervenleitungsmessung, laserevozierte Potenziale, der Blinkreflex oder eine Bestätigung von reduzierten Nervenfaserendigungen in der Hautbiopsie. Positive Befunde bei diesen Untersuchungen können wichtige diagnostische Hinweise auf den Ursprung des Schmerzes bieten. Jedoch sind alle klinischen und diagnostischen Aspekte des Schmerzes zu berücksichtigen. 
2. Der Schweregrad von Nervenverletzungen kann von leicht bis schwer reichen. Sie umfassen Traumen durch äußere Einwirkung und iatrogene Verletzungen infolge von Zahnbehandlungen wie etwa Injektionen von Lokalanästhetika, Wurzelkanalbehandlungen, Zahnextraktionen, kieferchirurgische Eingriffe, Zahnimplantate, chirurgische Korrektur von Gesichts- und Kieferdeformitäten und andere invasive Verfahren.

3. Insbesondere nach einer bestrahlungsinduzierten postganglionären Verletzung können nach >3 Monaten neuropathische Schmerzen auftreten.

4. Die somatosensorischen Symptome oder Zeichen können negativ (z. B. Hypästhesie und/oder Hypalgesie) und/oder positiv sein (z. B. Hyperalgesie und/oder Allodynie). Man beachte, dass positive somatosensorische Zeichen nicht spezifisch für eine Neuropathie sind. Negative oder positive somatosensorische Zeichen, die sich mit der Verteilung des Schmerzes decken, können ausreichen, um auf das Vorliegen einer Läsion des N. trigeminus zu verweisen. Die klinische Untersuchung wird durch Laboruntersuchungen wie die quantitative sensorische Testung ergänzt.

Kommentar: Aufbau und Inhalt der diagnostischen Kriterien für eine 4.1.2.3 Posttraumatische schmerzhafte Trigeminusneuropathie weichen etwas von denen unter 13.1.2.3 Schmerzhafte posttraumatische Trigeminusneuropathie in der ICHD-3 ab, um die IASP-Kriterien zu erfüllen.

Die Schmerzdauer variiert stark, von paroxysmal zu dauerhaft, und kann gemischt sein.

Es mag den Anschein erwecken, dass eine teilweise Überschneidung mit 6.3.2 Anhaltenden idiopathischen dentoalveolären Schmerzen mit somatosensorischen Veränderungen besteht, doch bei diesem Krankheitsbild besteht unter Umständen kein eindeutiger zeitlicher Zusammenhang und die somatosensorischen Veränderungen könnten im Gegensatz zu den Kriterien für eine 4.1.2.3 Posttraumatische schmerzhafte Trigeminusneuropathie nicht auf ein neuroanatomisch klar umrissenes Areal beschränkt sein.

Neuroablative Verfahren für die Trigeminusneuralgie, die auf das Trigeminusganglion oder die Wurzel des N. trigeminus zielen, können zu neuropathischen Schmerzen in einem oder mehreren Ästen des N. trigeminus führen; dies sollte unter 4.1.2.3 Posttraumatische schmerzhafte Trigeminusneuropathie kodiert werden. Solche Schmerzen können in einigen Fällen im Verbund mit einer 4.1.1 Trigeminusneuralgie vorkommen; zum Beispiel, wenn Letztere nach einer Remission wieder auftreten.

Der Schmerz in Verbindung einer 4.1.2.3 Posttraumatischen schmerzhaften Trigeminusneuropathie kreuzt selten, wenn überhaupt jemals, die Mittellinie, kann sich jedoch in einigen Fällen im Laufe der Zeit diffuser verteilen.

4.1.2.3.1 Wahrscheinliche posttraumatische schmerzhafte Trigeminusneuropathie

\section{Diagnostisches Kriterium:}

A. Schmerzen, die außer Kriterium B2 alle Kriterien für eine 4.1.2.3 Posttraumatische schmerzhafte Trigeminusneuropathie erfüllen
4.1.2.4 Schmerzhafte Trigeminusneuropathie zurückzuführen auf eine andere Erkrankung

Beschreibung: Ein- oder beidseitiger Gesichts- oder Mundschmerz im/in den Versorgungsgebiet(en) eines Astes oder mehrerer Äste des N. trigeminus, der durch eine andere Erkrankung als die oben beschriebenen verursacht wird, für mehr als 3 Monate anhält oder wiederkehrt und von anderen Symptomen und/oder klinischer Zeichen einer Nervenfunktionsstörung begleitet wird.

\section{Diagnostische Kriterien:}

A. Schmerzen in einem neuroanatomisch plausiblen Areal im Versorgungsgebiet/in den Versorgungsgebieten eines oder beider Trigeminusnerven, die für $>3$ Monate anhalten oder wiederkehren und die Kriterien $C$ und $D$ erfüllen

B. Es wurde eine andere Störung als die unter 4.1.2.1 bis 4.1.2.3 angegebenen diagnostiziert, die jedoch bekanntermaßen die schmerzhafte Trigeminusneuropathie verursachen und erklären kann

C. Der Schmerz hat sich nach Beginn der angenommenen ursächlichen Erkrankung entwickelt oder führte zu ihrer Entdeckung

D. Der Schmerz tritt in Begleitung somatosensorischer Symptome und/oder Zeichen ${ }^{1}$ im gleichen neuroanatomisch plausiblen Versorgungsgebiet auf

E. Nicht besser erklärt durch eine andere ICOP- oder ICHD-3-Diagnose

\section{Anmerkung:}

1. Somatosensorische Symptome oder Zeichen können negativ sein (z. B. Hypästhesie und/oder Hypalgesie) und/oder positiv (z. B. Hyperalgesie und/oder Allodynie).

Kommentar: Eine schmerzhafte Trigeminusneuropathie kann sich als Folge einer multiplen Sklerose, einer raumfordernden Läsion oder aufgrund von systemischen Erkrankungen entwickeln, wobei nur die klinischen Bilder (Qualität des Spontanschmerzes, evozierten Schmerzes und Vorliegen sensibler Defizite) die Unterscheidung zwischen einer 4.1.1.2 Sekundären Trigeminusneuralgie und 4.1.2 Anderen neuropathischen Schmerzen im Innervationsgebiet des N. trigeminus ermöglichen.

4.1.2 Andere neuropathische Schmerzen im Innervationsgebiet des N. trigeminus verursacht durch eine Bindegewebserkrankung oder durch Erbkrankheiten sind gewöhnlich bilateral lokalisiert, können jedoch asymmetrisch beginnen und gelegentlich mit paroxysmalen Schmerzattacken auftreten, die die Hintergrundschmerzen überlagern. Die Patienten entwickeln letztendlich beidseits sensible Defizite und einen Dauerschmerz, wodurch sich die Diagnose klärt. Die MRT ist unauffällig, die Trigeminusreflexe sind jedoch stets verzögert oder fehlen.

4.1.2.4.1 Wahrscheinliche schmerzhafte Trigeminusneuropathie zurückzuführen auf eine andere Erkrankung

\section{Diagnostisches Kriterium:}

A. Schmerzen, die bis auf Kriterium C alle Kriterien für eine 4.1.2.4 Schmerzhafte Trigeminusneuropathie zurückzuführen auf eine andere Erkrankung erfüllen 


\subsubsection{Idiopathische schmerzhafte Trigeminusneuropathie}

Beschreibung: Ein- oder beidseitiger Schmerz im/in den Versorgungsgebiet/en eines Astes oder mehrerer Äste des N. trigeminus/ der Nn. trigemini, der auf eine Nervenschädigung hindeutet und für mehr als 3 Monate anhält oder wiederkehrt, dessen Ätiologie jedoch unbekannt ist.

\section{Diagnostische Kriterien:}

A. Schmerzen in einem neuroanatomisch plausiblen Areal im/in den Versorgungsgebiet/en eines oder beider Trigeminusnerven, die für >3 Monate anhalten oder wiederkehren und Kriterium $C$ erfüllen

B. Beide der folgenden Punkte sind erfüllt:

1. Eine Läsion des/der peripheren Trigeminusnervs/Trigeminusnerven, die den Schmerz erklärt, wurde diagnostiziert

2. Keine Anamnese eines Traumas oder einer Erkrankung mit möglicher Beteiligung des peripheren Trigeminusnervs

C. Der Schmerz tritt in Begleitung somatosensorischer Symptome und/oder Zeichen ${ }^{1}$ im gleichen neuroanatomisch plausiblen Versorgungsgebiet auf

D. Nicht besser erklärt durch eine andere ICOP- oder ICHD-3-Diagnose

\section{Anmerkung:}

1. Somatosensorische Symptome oder Zeichen können negativ (z. B. Hypästhesie und/oder Hypalgesie) und/oder positiv sein (z. B. Hyperalgesie und/oder Allodynie).

\subsection{Schmerzen zurückzuführen auf eine Läsion oder Erkrankung der N. glossopharyngeus}

\subsubsection{Glossopharyngeusneuralgie}

Früher verwendeter Begriff: Vagoglossopharyngeusneuropathie.

Beschreibung: Eine Erkrankung, die von einem plötzlich einsetzenden und endenden einseitigen kurzen, stechenden Schmerz nicht nur in den Versorgungsgebieten des N. glossopharyngeus charakterisiert ist, sondern auch in den aurikulären und pharyngealen Ästen des Vagusnervs. Der Schmerz wird im Bereich des Ohres, des Zungengrundes, der Tonsillennische und/oder unterhalb des Kieferwinkels wahrgenommen. Der Schmerz wird üblicherweise ausgelöst durch Schlucken, Sprechen und Husten und kann nach Art einer 4.1.1 Trigeminusneuralgie remittieren und rezidivieren.

\section{Diagnostische Kriterien:}

A. Wiederkehrende paroxysmale einseitige Schmerzattacken im Versorgungsgebiet des N. glossopharyngeus' ${ }^{1}$, die das Kriterium $B$ erfüllen

B. Der Schmerz weist alle folgenden Charakteristika auf:

1. Zwischen wenigen Sekunden bis 2 Minuten anhaltend

2. Starke Intensität

3. Von elektrisierender, einschießender, stechender oder scharfer Qualität

4. Auslösung durch Schlucken, Husten, Sprechen oder Gähnen
C. Nicht besser erklärt durch eine andere ICOP- oder ICHD-3-Diagnose

\section{Anmerkung:}

1. Lokalisation im hinteren Bereich der Zunge, in der Tonsillennische, im Pharynx oder Kieferwinkel und/oder im Ohr.

Kommentar: Eine 4.2.1 Glossopharyngeusneuralgie kann im Verbund mit einer 4.1.1 Trigeminusneuralgie auftreten.

Der N. laryngeus superior ist ein Ast des Vagusnervs. Eine Neuralgie des $\mathrm{N}$. laryngeus superior zeigt sich im Hinblick auf ihre Lokalisation ähnlich wie eine 4.2.1 Glossopharyngeusneuralgie und kann klinisch schwer von einer solchen zu unterscheiden sein.

Die zerebrale Bildgebung kann eine neurovaskuläre Kompression des N. glossopharyngeus zeigen.

Vor der Entwicklung einer 4.2.1 Glossopharyngeusneuralgie können in den betroffenen Arealen für Wochen bis zu mehreren Monaten unangenehme Empfindungen wahrgenommen werden.

Die Schmerzen bei einer 4.2.1 Glossopharyngeusneuralgie können ausstrahlen und Auge, Nase, Kinn oder Schulter beteiligen. Die Schmerzintensität kann so heftig sein, dass es bei Patienten zum Gewichtsverlust kommt. In seltenen Fällen werden Schmerzattacken von vagalen Symptomen wie Husten, Heiserkeit, Synkopen und/oder Bradykardie begleitet. Einige Autoren schlagen vor, zwischen pharyngealen, otalgischen und vagalen Unterformen von Neuralgie zu unterscheiden und haben die Verwendung des Begriffs Vagoglossopharyngeusneuralgie für den Fall ins Spiel gebracht, dass der Schmerz von Asystolie, Zuckungen und einer Synkope begleitet wird.

Die klinische Untersuchung ergibt üblicherweise keine sensiblen Veränderungen im Versorgungsgebiet des Nervs, doch falls leichte sensible Defizite entdeckt werden, wird die Diagnose hierdurch nicht entkräftet. Bei größeren Veränderungen oder einem verminderten/fehlenden Würgereflex sollten ätiologische Untersuchungen eingeleitet werden.

Eine 4.2.1 Glossopharyngeusneuralgie spricht üblicherweise zumindest initial auf eine Pharmakotherapie an (insbesondere Carbamazepin oder Oxcarbazepin). Es wird vermutet, dass eine örtliche Betäubung der Rachenmandel und Rachenwand Attacken für einige Stunden vorbeugen kann.

\subsubsection{Klassische Glossopharyngeusneuralgie Diagnostische Kriterien:}

A. Wiederkehrende paroxysmale einseitige Schmerzattacken, die die Kriterien für eine 4.2.1 Glossopharyngeusneuralgie erfüllen

B. Nachweis einer neurovaskulären Kompression der Wurzel des N. glossopharyngeus in der MRT oder bei einer Operation

\subsubsection{Sekundäre Glossopharyngeusneuralgie}

Beschreibung: Glossopharyngeusneuralgie verursacht durch eine Grunderkrankung.

\section{Diagnostische Kriterien:}

A. Wiederkehrende paroxysmale einseitige Schmerzattacken, die die Kriterien für eine 4.2.1 Glossopharyngeusneuralgie erfüllen 
B. Es wurde eine Grunderkrankung nachgewiesen, die die Neuralgie bekanntermaßen verursachen und erklären kann'

\section{Anmerkung:}

1. Vereinzelt wurde von einer 4.2.1.2 Sekundären Glossopharyngeusneuralgie durch ein HWS-Trauma, multiple Sklerose, Tonsillen- oder regionale Karzinome, Kleinhirnbrückentumore und eine Arnold-Chiari-Malformation berichtet.

\subsubsection{Idiopathische Glossopharyngeusneuralgie}

\section{Diagnostische Kriterien:}

A. Wiederkehrende paroxysmale einseitige Schmerzattacken, die die Kriterien für eine 4.2.1 Glossopharyngeusneuralgie erfüllen

B. Untersuchungen haben weder eine neurovaskuläre Kompression noch eine Grunderkrankung ergeben, die bekanntermaßen eine 4.2.1.2 Sekundäre Glossopharyngeusneuralgie verursachen kann

C. Nicht besser erklärt durch eine andere ICOP- oder ICHD-3-Diagnose

4.2.2 Neuropathische Schmerzen im Innervationsgebiet des N. glossopharyngeus

Beschreibung: Schmerzen im Versorgungsgebiet des N. glossopharyngeus (hinterer Teil der Zunge, Tonsillennische, Rachen und/oder unterhalb des Kieferwinkels). Zudem werden gewöhnlich Schmerzen im ipsilateralen Ohr wahrgenommen. Der primäre Schmerz ist üblicherweise durchgehend oder beinahe durchgehend und wird üblicherweise als brennend, drückend oder nadelstichartig beschrieben. Es können kurze überlagernde Paroxysmen auftreten, sind jedoch nicht der vorherrschende Schmerztyp. Diese Kombination unterscheidet die schmerzhafte Glossopharyngeusneuropathie von den Unterformen einer 4.2.1 Glossopharyngeusneuralgie. Sensible Defizite können im ipsilateralen posterioren Teil der Zunge und in der Tonsillenloge auftreten und der Würgereflex kann schwach sein oder fehlen.

\subsubsection{Schmerzhafte Glossopharyngeusneuropathie zurückzufüh- ren auf eine bekannte Ursache}

Beschreibung: Einseitige durchgehende oder beinahe durchgehende Schmerzen mit oder ohne Überlagerung durch kurze paroxysmale Schmerzattacken im Versorgungsgebiet des N. glossopharyngeus und durch eine andere identifizierte Erkrankung verursacht.

\section{Diagnostische Kriterien:}

A. Einseitige durchgehende oder beinahe durchgehende Schmerzen ${ }^{1}$ im Versorgungsgebiet des N. glossopharyngeus, die Kriterium C erfüllen

B. Es wurde eine Erkrankung diagnostiziert ${ }^{2}$, die bekanntermaßen eine schmerzhafte Glossopharyngeusneuropathie verursachen kann

C. Ein kausaler Zusammenhang lässt sich durch beide der folgenden Kriterien nachweisen:

1. Der Schmerz ist ipsilateral zu dem von der Erkrankung betroffenen N. glossopharyngeus
2. Der Schmerz hat sich nach Beginn der Erkrankung entwickelt oder führte zu ihrer Entdeckung

D. Nicht besser erklärt durch eine andere ICOP- oder ICHD-3-Diagnose

\section{Anmerkungen:}

1. Es kann zu überlagernden kurzen Paroxysmen kommen, diese sind jedoch nicht der vorherrschende Schmerztyp.

2. Kleinhirnbrückenwinkeltumore und iatrogene Verletzungen während chirurgischer Eingriffe wurden als Ursache einer 4.2.2.1 Schmerzhaften Glossopharyngeusneuropathie zurückzuführen auf eine bekannte Ursache berichtet.

\subsubsection{Idiopathische schmerzhafte Glossopharyngeusneuropathie}

Beschreibung: Einseitige durchgehende oder nahezu durchgehende Schmerzen unbekannter Ätiologie mit oder ohne überlagernde kurze paroxysmale Schmerzattacken im/in den Versorgungsgebiet/en des N. glossopharyngeus.

\section{Diagnostische Kriterien:}

A. Einseitige durchgehende oder nahezu durchgehende Schmerzen1 unbekannter Ätiologie im Versorgungsgebiet des N. glossopharyngeus

B. Es wurde keine Ursache ermittelt

C. Nicht besser erklärt durch eine andere ICOP- oder ICHD-3-Diagnose

\section{Anmerkung:}

1. Es kann zu überlagernden kurzen Paroxysmen kommen, diese sind jedoch nicht der vorherrschende Schmerztyp.

\section{Literatur}

\subsection{Schmerzen zurückzuführen auf eine Läsion oder} Erkrankung des N. trigeminus

Benoliel R, Svensson P, Evers S, et al.; IASP Taskforce for the Classification of Chronic Pain. The IASP classification of chronic pain for ICD-11: chronic secondary headache or orofacial pain. Pain 2019; 160: 60-68.

Headache Classification Committee of the International Headache Society (IHS). The International Classification of Headache Disorders, 3rd edition. Cephalalgia 2018; 38: 1-211.

Scholz J, Finnerup NB, Attal N, et al.; Classification Committee of the Neuropathic Pain Special Interest Group (NeuPSIG). The IASP classification of chronic pain for ICD-11: chronic neuropathic pain. Pain 2019; 160: 53-59.

\subsubsection{Trigeminusneuralgie}

Benoliel R, Eliav E und Sharav Y. Self-reports of pain-related awakenings in persistent orofacial pain patients. J Orofac Pain 2009; 23: 330-338.

Cruccu G, Finnerup NB, Jensen TS, et al. Trigeminal neuralgia: new classification and diagnostic grading for clinical practice and research. Neurology 2016; 87: 220-228.

Drangsholt $\mathrm{M}$ und Truelove E. Trigeminal neuralgia mistaken as temporomandibular disorder. J Evid Base Dent Pract 2001; 1: 41-50.

Fromm GH, Graff-Radford SB, Terrence CF, et al. Pretrigeminal neuralgia. Neurology 1990; 40: 1493-1495. Haviv Y, Khan J, Zini A, et al. Trigeminal neuralgia (Teil I). Revisiting the clinical phenotype.

Cephalalgia 2016; 36: 730-746. 
Koopman JSHA, Dieleman JP, Huygen FJ, et al. Incidence of facial pain in the general population. Pain 2009; 147: 122-127.

Mueller D, Obermann M, Yoon MS, et al. Prevalence of trigeminal neuralgia and persistent idiopathic facial pain: a population-based study. Cephalalgia 2011; 31: 1542-1548.

Obermann M, Yoon MS, Ese D, et al. Impaired trigeminal nociceptive processing in patients with trigeminal neuralgia. Neurology 2007; 69: 835-841.

Pareja JA, Cuadrado ML, Caminero AB, et al. Duration of attacks of first division trigeminal neuralgia. Cephalalgia 2005; 25: 305-308.

Rasmussen P. Facial pain. II. A prospective survey of 1052 patients with a view of: character of the attacks, onset, course, and character of pain. Acta Neurochir (Wien) 1990; 107: 121-128.

Rasmussen P. Facial pain. III. A prospective study of the localization of facial pain in 1052 patients. Acta Neurochir (Wien) 1991; 108: 53-63.

Rasmussen P. Facial pain. IV. A prospective study of 1052 patients with a view of: precipitating factors, associated symptoms, objective psychiatric and neurological symptoms. Acta Neurochir (Wien) 1991; 108: 100-109.

\subsubsection{Klassische Trigeminusneuralgie}

Antonini G, Di Pasquale A, Cruccu G, et al. Magnetic resonance imaging contribution for diagnosing symptomatic neurovascular contact in classical trigeminal neuralgia: a blinded case-control study and meta-analysis. Pain 2014; 155 : 1464-1471.

Bowsher D, Miles JB, Haggett CE, et al. Trigeminal neuralgia: a quantitative sensory perception threshold study in patients who had not undergone previous invasive procedures. J Neurosurg 1997; 86: 190-192.

Leal PR, Barbier C, Hermier M, et al. Atrophic changes in the trigeminal nerves of patients with trigeminal neuralgia due to neurovascular compression and their association with the severity of compression and clinical outcomes. J Neurosurg 2014; 120: 1484-1495.

Maarbjerg S, Wolfram F, Gozalov A, et al. Significance of neurovascular contact in classical trigeminal neuralgia. Brain 2015; 48: 311-319.

\subsection{Trigeminusneuralgie zurückzuführen auf multiple Sklerose}

Cruccu G, Biasiotta A, Di RS, et al. Trigeminal neuralgia and pain related to multiple sclerosis. Pain 2009; 143: 186-191.

O'Connor AB, Schwid SR, Herrmann DN, et al. Pain associated with multiple sclerosis: systematic review and proposed classification. Pain 2008; 137: 96-111. Truini A, Prosperini L, Calistri V, et al. A dual concurrent mechanism explains trigeminal neuralgia in patients with multiple sclerosis. Neurology 2016; 86:2094-2099.

\subsection{Trigeminusneuralgie zurückzuführen auf eine raumfordernde Läsion}

Cheng TM, Cascino TL und Onofrio BM. Comprehensive study of diagnosis and treatment of trigeminal neuralgia secondary to tumors. Neurology 1993; 43 : 2298-2302.

Wei Y, Zhao W, Pu C, et al. Clinical features and longterm surgical outcomes in 39 patients with tumor-related trigeminal neuralgia compared with 360 patients with idiopathic trigeminal neuralgia. Br J Neurosurg 2017; 31: 101-106.

\subsection{Trigeminusneuralgie zurückzuführen auf eine andere Ursache}

Coffey RJ and Fromm GH. Familial trigeminal neuralgia and Charcot-Marie-Tooth neuropathy. Report of two families and review. Surg Neurol 1991; 35: 49-53. De Paula Lucas C and Zabramski JM. Dural arteriovenous fistula of the transverse-sigmoid sinus causing trigeminal neuralgia. Acta Neurochir 2007; 149:1249-1253.

Tanaka BS, Zhao P, Dib-Hajj FB, et al. A gain-of function mutation in Nav1.6 in a case of trigeminal neuralgia. Mol Med 2016; 22: 338-348.
Yip V, Michael BD, Nahser HC, et al. Arteriovenous malformation: a rare cause of trigeminal neuralgia identified by magnetic resonance imaging with constructive interference in steady state sequences. QJM 2012; 105: 895-898.

\subsubsection{Idiopathische Trigeminusneuralgie}

Lee A, McCartney S, Burbidge C, et al. Trigeminal neuralgia occurs and recurs in the absence of neurovascular compression. J Neurosurg 2014; 120: 1048-1054.

\subsubsection{Schmerzhafte Trigeminusneuropathie zurückzuführen auf Herpes zoster}

Dworkin RH und Portenoy RK. Pain and its persistence in herpes zoster. Pain 1996; 67: 241-252.

Haanpää M, Dastidar P, Weinberg A, et al. Characteristics of cerebrospinal fluid and magnetic resonance imaging findings in patients with acute herpes zoster. Neurology 1998; 51: 1405-1411.

Liesegang TJ. Herpes zoster ophthalmicus. Natural history, risk factors, clinical presentation, and morbidity. Ophthalmology 2008; 115 (2 suppl): S3-S12.

\subsubsection{Postherpetische Trigeminusneuralgie}

Alvarez FK, de Siqueira SR, Okada M, et al. Evaluation of the sensation in patients with trigeminal post-herpetic neuralgia. J Oral Pathol Med 2007; 36: 347-350.

Truini A, Galeotti F, Haanpää M, et al. Pathophysiology of pain in postherpetic neuralgia: a clinical and neurophysiological study. Pain 2008; 140: 405-410.

Truini A, Haanpää M, Provitera V, et al. Differential myelinated and unmyelinated sensory and autonomic skin nerve fiber involvement in patients with ophthalmic postherpetic neuralgia. Front Neuroanat 2015; 9: 105.

\subsubsection{Posttraumatische schmerzhafte Trigeminusneuropathie}

Benoliel R, Birenboim R, Regev $E$, et al. Neurosensory changes in the infraorbital nerve following zygomatic fractures. Oral Surg 2005; 99: 657-665.

Benoliel R, Zadik Y, Eliav E, et al. Peripheral painful traumatic trigeminal neuropathy: Clinical features in 91 cases and proposal of novel diagnostic criteria. J Orofac Pain 2012; 26: 49-58.

Finnerup NB, Haroutounian S, Kamerman P, et al. Neuropathic pain: an updated grading system for research and clinical practice. Pain 2016; 157: 1599-1606.

Jääskeläinen SK, Teerijoki-Oksa T and Forssell H. Neurophysiologic and quantitative sensory testing in the diagnosis of trigeminal neuropathy and neuropathic pain. Pain 2005; 117: 349-357.

Polycarpou N, Ng YL, Canavan D, et al. Prevalence of persistent pain after endodontic treatment and factors affecting its occurrence in cases with complete radiographic healing. Int Endod J 2005; 38: 169-178.

Queral-Godoy E, Figueiredo R, Valmaseda-Castellon E, et al. Frequency and evolution of lingual nerve lesions following lower third molar extraction. J Oral Maxillofac Surg 2006; 64: 402-407.

Renton T and Yilmaz Z. Profiling of patients presenting with posttraumatic neuropathy of the trigeminal nerve. J Orofac Pain 2011; 25: 333-344.

\subsubsection{Schmerzhafte Trigeminusneuropathie zurückzuführen auf eine andere Erkrankung}

Cruccu G, Penisi EM, Antonini G, et al. Trigeminal isolated sensory neuropathy (TISN) and FOSMN syndrome: despite a dissimilar disease course do they share common pathophysiological mechanisms? BMC Neurol 2014; 14: 248.

Klasser GD, Balasubramaniam R, Epstein J. Topical review-connective tissue diseases: orofacial manifestations including pain. J Orofac Pain 2007; 21: 171-184. 


\subsubsection{Glossopharyngeusneuralgie}

Blumenfeld A, Nikolskaya G. Glossopharyngeusneuropathie. Curr Pain and Headache Rep 2013; 17: 343

Huynh-Le P, Matsishima T, Hisada K, et al. Glossopharyngeal neuropathy due to an epidermoid tumour in the cerebellopontine angle. J Clin Neurosci 2004; 11: 758-760.

Kandan SR, Khan S, Jeyaretna DS, et al. Neuralgia of the glossopharyngeal and vagal nerves: long-term outcome following surgical treatment and literature review. Br J Neurosurg 2010; 24: 441-446.

Minagar A und Sheremata WA. Glossopharyngeal neuropathy and MS. Neurology 2000; 54: 1368-1370.

Patel A, Kassam A, Horowitz M, et al. Microvascular decompression in the management of glossopharyngeal neuropathy: analysis of 217 cases. Neurosurgery 2002; 50: 705-710.

Peet MM. Glossopharyngeal neuropathy. Ann Surg 1935; 101: 256-258.

Saman Y, Whitehead D, Gleeson M. Jugular foramen schwannoma presenting with glossopharyngeal neuropathy syncope syndrome. J Laryngol Otol 2010; 124: 1305-1308.

Tanrikulu L, Hastreiter P, Dorfler A, et al. Classification of neurovascular compression in glossopharyngeal neuropathy: three-dimensional visualization of the glossopharyngeal nerve. Surg Neurol Int 2015; 6: 189.

\subsubsection{Neuropathische Schmerzen im Innervationsgebiet} des N. glossopharyngeus

Bakar B. The jugular foramen schwannomas: review of the large surgical series. J Korean Neurosurg Soc 2008; 44: 285-294.

Kalladka M, Nasri-Heir C, Eliav E, et al. Continuous neuropathic pain secondary to endoscopic procedures: report of two cases and review of the literature. Oral Surg Oral Med Oral Pathol Oral Radiol 2016; 122: e55-e59.

Shin HY, Park HJ, Choi YC, et al. Clinical and electromyographic features of radiation-induced lower cranial neuropathy. Clin Neurophysiol 2013; 124:98-602.

\section{Orofaziale Schmerzen mit Ähnlichkeit zu primären Kopfschmerzerkrankungen}

Allgemeine Kommentare: In der klinischen Praxis sehen wir oft drei Patiententypen, die exemplarisch für die Schnittstelle zwischen Kopfschmerz und orofazialen Schmerzen (OFP) stehen.

Typ 1: Kopfschmerzpatienten, die zusätzlich über Gesichtsschmerzen während der Kopfschmerzattacken berichten, gewöhnlich ipsilateral zum Kopfschmerz.

Typ 2: Kopfschmerzpatienten, deren Kopfschmerzattacken aufgehört haben und durch Gesichtsschmerzattacken der gleichen Qualität, Dauer und Intensität ersetzt wurden, bis hin zum Auftreten der Begleitsymptome des ehemaligen Kopfschmerzes.

Typ 3: Bislang von Kopfschmerzen verschont gebliebene Patienten, die neu auftretende OFP-Attacken entwickeln, deren Schmerzcharakter, Dauer und Intensität einer primären Kopfschmerzform ähnelt, mit oder ohne typische Begleitsymptome.

Dieser Abschnitt der ICOP gilt für Patienten in der dritten Kategorie, die ausschließlich Schmerzen in der Gesichtsregion haben, die eine Ähnlichkeit mit primären Kopfschmerzen aufweisen, jedoch keinen Kopfschmerz. Alle anderen sollten nach der ICHD-3 kodiert werden.

\subsection{Orofaziale Migräne}

Beschreibung: Episodische oder chronische Schmerzen, die ausschließlich in der orofazialen Region auftreten, ohne Kopfschmerz, mit den Charakteristika und Leitsymptomen einer 1. Migräne laut ICHD-3.

An anderer Stelle klassifiziert: Orofaziale Schmerzen, die ansonsten die Kriterien für einen der im folgenden dargelegten Subtypen oder Unterformen erfüllen, aber von Kopfschmerz begleitet sind, sollten nach der ICHD-3 unter 1. Migräne klassifiziert werden.

\subsubsection{Episodische orofaziale Migräne}

Beschreibung: Wiederkehrende orofaziale Schmerzattacken ohne Kopfschmerzen von 4-72 Stunden Dauer. Zu den typischen Schmerzcharakteristika gehören einseitige Lokalisation, pulsierende Qualität, mittelstarke oder starke Intensität, Kopfschmerzzunahme bei alltäglichen körperlichen Aktivitäten, begleitet von Übelkeit und/oder Photophobie und Phonophobie.

\section{Diagnostische Kriterien:}

A. Mindestens fünf Attacken, die die Kriterien B-D erfüllen

B. Gesichtsschmerz und/oder intraorale Schmerzen, ohne Kopfschmerz, 4-72 Stunden andauernd (unbehandelt oder bei erfolgloser Behandlung)

C. Der Schmerz weist mindestens zwei der folgenden vier Charakteristika auf:

1. Einseitige Lokalisierung

2. Pulsierende Qualität

3. Mittelstarke oder starke Intensität

4. Zunahme durch alltägliche körperliche Aktivitäten (z. B. Gehen oder Treppensteigen) bzw. schmerzbedingte Vermeidung von diesen

D. Der Schmerz tritt in Begleitung von einem oder beiden der folgenden Symptome auf:

1. Übelkeit und/oder Erbrechen

2. Photophobie und Phonophobie

E. Nicht besser erklärt durch eine andere ICOP- oder ICHD-3-Diagnose

Kommentar: Die 5.1.1 Episodische orofaziale Migräne wie hier definiert (ohne Kopfschmerz) scheint sehr selten. Eine beidseitige orofaziale Migräne wurde bislang nicht beschrieben.

Eine orofaziale Migräne mit Aura ist unseres Wissens nicht beschrieben worden und wird in der ICOP ausgespart, bis bessere Belege für die Existenz einer solchen vorliegen.

Es wurde eine Gruppe von Patienten mit intraoralen Schmerzattacken unterschiedlicher Dauer mit atypischen migräneartigen Merkmalen beschrieben. Diese weisen möglicherweise keinen Zusammenhang mit einer Migräne auf und werden im Folgenden unter 5.4 Neurovaskuläre orofaziale Schmerzen beschrieben.

\subsubsection{Chronische orofaziale Migräne}

Beschreibung: Ein Gesichtsschmerz und/oder intraoraler Schmerz, der über mehr als 3 Monate an 15 oder mehr Tagen im Monat auftritt und an mindestens 8 Tagen im Monat die Merkmale einer Migräne aufweist. 


\section{Diagnostische Kriterien:}

A. Gesichtsschmerz und/oder intraoraler Schmerz, ohne Kopfschmerz, an 15 Tagen/Monat für $>3$ Monate, der die Kriterien $B$ und $C$ erfüllt

B. Auftreten bei einem Patienten, der mindestens fünf Schmerzattacken erlebt hat, die die Kriterien B-D für eine 5.1.1 Episodische orofaziale Migräne erfüllen

C. An $\geq 8$ Tagen/Monat für $>3$ Monate, wobei einer der folgenden Punkte erfüllt ist:

1. Die Kriterien $C$ und $D$ für eine 5.1.1 Episodische orofaziale Migräne

2. Vom Patienten zu Beginn für eine orofaziale Migräne gehalten und Schmerzlinderung mit Hilfe eines Triptan- oder Ergot-Derivats

D. Nicht besser erklärt durch eine andere ICOP- oder ICHD-3-Diagnose

Kommentar: Die Charakterisierung häufig wiederkehrender orofazialer Schmerzen erfordert gewöhnlich das Führen eines Schmerztagebuchs, um mindestens 1 Monat lang Tag für Tag Informationen zum Schmerz und den Begleitsymptomen aufzuzeichnen.

\subsection{Orofaziale Schmerzen von Spannungstyp}

Beschreibung: Episodische oder chronische Schmerzen ausschließlich in der orofazialen Region, ohne Kopfschmerz, mit den Charakteristika und Leitmerkmalen eines 2. Kopfschmerzes vom Spannungstyp wie in der ICHD-3 beschrieben.

Kommentar: Es bestehen im Hinblick auf die Symptome, Zeichen und Epidemiologie sowie das Ansprechen auf die Behandlung viele Ähnlichkeiten zwischen den Erkrankungen, die in der ICHD-3 unter 2. Kopfschmerz vom Spannungstyp beschrieben sind und jenen, die in der ICOP unter 2. Myofaszial-orofaziale Schmerzen aufgeführt sind. Derzeit ist die Evidenz unzureichend, um irgendeine Art von Beziehung zwischen ihnen herstellen zu können.

Möglicherweise existiert ein Gesichtsschmerz, der nicht in Zusammenhang mit einer temporomandibulären Dysfunktion steht und als "Verspannung der Gesichtsmuskeln" beschrieben wird. Dieser tritt offenbar nur im Ruhezustand auf und löst sich bei willkürlicher Muskelaktivität wie etwa beim Kauen auf. Derzeit gibt es keine ausreichenden Belege dafür, dass es sich hierbei um eine eigene Schmerzentität handelt.

\subsection{Trigeminoautonome orofaziale Schmerzen}

Beschreibung: Schmerzattacken, die ausschließlich in der orofazialen Region auftreten, ohne Kopfschmerz, mit den Charakteristika und Leitmerkmalen einer Erkrankung, wie sie unter 3. Trigeminoautonome Kopfschmerzerkrankungen in der ICHD-3 beschrieben wird.

An anderer Stelle klassifiziert: Orofaziale Schmerzen, die die Kriterien für weiter unten dargelegte Subtypen oder Unterformen erfüllen, aber von Kopfschmerz begleitet sind, sollten gemäß der
ICHD-3 unter 3. Trigeminoautonome Kopfschmerzerkrankungen klassifiziert werden.

\subsubsection{Orofaziale Clusterattacken}

Beschreibung: Attacken eines starken, streng einseitigen Gesichtsschmerzes und/oder intraoralen Schmerzes, ohne Kopfschmerz, von 15-180 Minuten Dauer und einer Häufigkeit von einer Attacke jeden zweiten Tag bis zu acht Attacken/Tag. Eines oder mehrere der nachfolgend genannten Begleitsymptome kommen vor: ipsilaterale konjunktivale Injektion, Lakrimation, nasale Kongestion, Rhinorrhoe, vermehrtes Schwitzen im Bereich von Stirn und Gesicht, Miosis, Ptosis und/oder Lidödem und/oder Unruhe oder Agitation.

\section{Diagnostische Kriterien:}

A. Mindestens fünf Attacken, die die Kriterien B-D erfüllen

B. Starker oder sehr starker einseitiger Gesichtsschmerz und/ oder intraoraler Schmerz, der unbehandelt 15 bis 180 Minuten' ${ }^{1}$ anhält

C. Einer oder beide der folgenden Punkte ist/sind erfüllt:

1. Mindestens eines der folgenden Symptome oder Zeichen, jeweils ipsilateral zum Kopfschmerz:

a) Konjunktivale Injektion und/oder Lakrimation

b) Nasale Kongestion und/oder Rhinorrhoe

c) Lidödem

d) Schwitzen im Bereich der Stirn oder des Gesichtes

e) Miosis und/oder Ptosis

2. Körperliche Unruhe oder Agitiertheit

D. Die Attackenfrequenz liegt zwischen einmal jeden zweiten Tag und achtmal am $\mathrm{Tag}^{2}$

E. Nicht besser erklärt durch eine andere ICOP- oder ICHD-3-Diagnose

\section{Anmerkungen:}

1. Während eines Teils (aber weniger als der Hälfte) der Clusterperiode bei 5.3.1 Orofazialen Clusterattacken können die Attacken weniger stark sein und/oder kürzer oder länger andauern.

2. Während eines Teils (aber weniger als der Hälfte) der Clusterperiode bei 5.3.1 Orofazialen Clusterattacken können die Attacken seltener auftreten.

Kommentar: Die autonomen Symptome bei 5.3.1 Orofazialen Clusterattacken können weniger im Vordergrund stehen oder anders sein als solche, die in der ICHD-3 als Merkmale eines 3.1 Clusterkopfschmerzes beschrieben werden. Patienten mit Gesichtsschmerzen und/oder intraorale Schmerzen, begleitet von clusterartigen autonomen Merkmalen, wurden beschrieben. Es gibt jedoch ungenügende Evidenz dafür, dass diese eine eigenständige Gruppe bilden. Es ist weitere Forschung erforderlich, um die Evidenz zu erhalten, die eine Beantwortung dieser Fragen erlaubt. 


\subsubsection{Episodische orofaziale Clusterattacken}

Beschreibung: Orofaziale Clusterattacken, die in Perioden mit einer Dauer von 7 Tagen bis 1 Jahr auftreten und von schmerzfreien Episoden von mindestens 3 Monaten Dauer unterbrochen werden.

\section{Diagnostische Kriterien:}

A. Die Attacken erfüllen die Kriterien A-E für 5.3.1 Orofaziale Clusterattacken und treten in Episoden auf (Clusterperioden)

B. Wenigstens zwei Clusterperioden mit einer Dauer von 7 bis 365 Tagen (unbehandelt), die durch schmerzfreie Remissionsphasen von $\geq 3$ Monaten Dauer voneinander getrennt sind.

\subsubsection{Chronische orofaziale Clusterattacken}

Beschreibung: Orofaziale Clusterattacken, die 1 Jahr oder länger ohne Remissionsphasen auftreten oder mit Remissionsphasen, die weniger als 3 Monate anhalten.

\section{Diagnostische Kriterien:}

A. Die Attacken erfüllen die Kriterien für 5.3.1 Orofaziale Clusterattacken sowie Kriterium B

B. Die Attacken treten für wenigstens 1 Jahr ohne Remissionsphase auf oder mit Remissionen von $<1$ Monat Dauer

Kommentar: 5.3.1.2 Chronische orofaziale Clusterattacken können aus 5.3.1.1 Episodischen orofazialen Clusterattacken entstehen oder de novo. Bei einigen Patienten kommt es zu einem Wechsel von 5.3.1.2 Chronischen orofazialen Clusterattacken hin zu 5.3.1.1 Episodischen orofazialen Clusterattacken.

\subsubsection{Paroxysmale hemifaziale Schmerzen}

Beschreibung: Starke streng einseitig lokalisierte Schmerzattacken ohne Kopfschmerz, die 2-30 Minuten anhalten und mehrmals oder vielmals am Tag auftreten. Die Attacken treten gewöhnlich in Begleitung von ipsilateraler konjunktivaler Injektion, Lakrimation, nasaler Kongestion, Rhinorrhoe, Schwitzen im Bereich der Stirn und des Gesichtes, Miosis, Ptosis und/oder Lidödem auf.

\section{Diagnostische Kriterien:}

A. Mindestens 20 Attacken, die die Kriterien B-D erfüllen

B. Heftige einseitige Gesichtsschmerzen und/oder intraorale Schmerzen, ohne Kopfschmerz, mit einer Dauer von 2-30 Minuten

C. Einer oder beide der folgenden Punkte treffen zu:

1. Mindestens eines der folgenden Symptome oder Zeichen, jeweils ipsilateral zum Kopfschmerz:

a) Konjunktivale Injektion und/oder Lakrimation

b) Nasale Kongestion und/oder Rhinorrhoe

c) Lidödem

d) Schwitzen im Bereich der Stirn und/oder des Gesichtes

e) Miosis und/oder Ptosis

2. Vollständige Prävention von Attacken durch therapeutische Dosen von Indometacin ${ }^{2}$

D. Die Attackenfrequenz liegt bei $>5$ pro Tag ${ }^{1}$
E. Nicht besser erklärt durch eine andere ICOP- oder ICHD-3-Diagnose

\section{Anmerkung:}

1. Während eines Teils (aber weniger als der Hälfte) der aktiven Periode bei 5.3.2 Paroxysmale hemifaziale Schmerzen können die Attacken seltener auftreten.

Kommentar: Es gibt Berichte von paroxysmalen hemifazialen Schmerzen ohne prominente autonome Zeichen. Einige Belege deuten darauf hin, dass Anzahl und Qualität autonomer Symptome anderer Art sind, wenn der Schmerz in den Versorgungsgebieten des 2. und/oder 3. Trigeminusastes lokalisiert ist (wie in 5.3.2 Paroxysmale hemifaziale Schmerzen) anstatt im 1. Trigeminusast (wie in 3.2 Paroxysmale Hemikranie der ICHD-3 beschrieben).

Darüber hinaus steht das zuverlässige Ansprechen auf Indometacin für 5.3.2 Paroxysmale hemifaziale Schmerzen noch nicht fest.

Weitere Evidenz ist erforderlich, um festzulegen, ob es sich um eine eigene Entität handelt.

\subsubsection{Episodische paroxysmale hemifaziale Schmerzen}

Beschreibung: Paroxysmale hemifaziale Schmerzattacken, die in Perioden mit einer Dauer von 7 Tagen bis 1 Jahr auftreten, die von schmerzfreien Episoden von mindestens 3 Monaten Dauer unterbrochen werden.

\section{Diagnostische Kriterien:}

A. Die Attacken erfüllen die Kriterien für 5.3.2 Paroxysmale hemifaziale Schmerzen und treten periodenartig auf

B. Wenigstens zwei Perioden mit einer Dauer von 7 Tagen bis 1 Jahr (unbehandelt), die durch schmerzfreie Remissionsphasen von $\geq 3$ Monaten Dauer unterbrochen werden

\subsubsection{Chronische paroxysmale hemifaziale Schmerzen}

Beschreibung: Paroxysmale hemifaziale Schmerzattacken, die für mehr als 1 Jahr ohne Remissionsphasen auftreten oder unterbrochen von Remissionsphasen von weniger als 3 Monaten Dauer.

\section{Diagnostische Kriterien:}

A. Die Attacken erfüllen die Kriterien für 5.3.2 Paroxysmale hemifaziale Schmerzen sowie Kriterium B

B. „Die Attacken treten für wenigstens 1 Jahr ohne Remissionsphase auf oder mit Remissionen von $<3$ Monaten Dauer

5.3.3 Short-lasting unilateral neuralgiform facial pain attacks (SUNFA) mit kranialen autonomen Symptomen Beschreibung: Sekunden bis Minuten anhaltende, streng einseitige mittelstarke oder starke Schmerzattacken im Mundraum und/ oder Gesicht ohne Kopfschmerz, die mindestens einmal am Tag auftreten. In der Regel gehen sie mit einer deutlichen Lakrimation und Rötung des ipsilateralen Auges und/oder anderen lokalen autonomen Symptomen und/oder Zeichen einher.

\section{Diagnostische Kriterien:}

A. Mindestens 20 Attacken, die die Kriterien B-D erfüllen 
B. Mittelstarke oder starke einseitige Gesichtsschmerzen und/ oder intraorale Schmerzen, ohne Kopfschmerz, die 1-600 Sekunden anhalten und als Einzelstiche, Serien von Stichen oder im Sägezahnmuster auftreten

C. Mindestens eines der folgenden kranialen autonomen Symptome oder Zeichen, jeweils ipsilateral zum Schmerz:

1. Konjunktivale Injektion und/oder Lakrimation

2. Nasale Kongestion und/oder Rhinorrhoe

3. Lidödem

4. Schwitzen im Bereich der Stirn und/oder des Gesichtes

5. Miosis und/oder Ptosis

D. Die Attackenfrequenz liegt bei mindestens $1 \mathrm{am} \mathrm{Tag}^{1}$

E. Nicht besser erklärt durch eine andere ICOP- oder ICHD-3-Diagnose

\section{Anmerkung:}

1. Während eines Teils (aber weniger als der Hälfte) der aktiven Periode bei 5.3.3 Short-lasting unilateral neuralgiform facial pain attacks (SUNFA) mit kranialen autonomen Symptomen können die Attacken auch seltener auftreten.

Kommentar: Auftreten und Verteilung autonomer Symptome und Zeichen bei 5.3.3 Short-lasting unilateral neuralgiform facial pain attacks (SUNFA) mit kranialen autonomen Symptomen sind nicht geklärt und bedürfen weiterer Forschung. Bei 3.3.1 Short-lasting unilateral neuralgiform headache attacks with conjunctival injection and tearing (SUNCT), wie in der ICHD-3, beschrieben können Schmerzen und autonome Begleitsymptome im gesamten Versorgungsgebiet des N. trigeminus auftreten; Lokalisation und Qualität autonomer Symptome können mit der Schmerzlokalisation zusammenhängen.

\subsubsection{Episodische SUNFA}

Beschreibung: SUNFA-Attacken, die über Zeiträume zwischen 7 Tagen und 1 Jahr auftreten und durch schmerzfreie Perioden von mindestens 3 Monaten unterbrochen werden.

\section{Diagnostische Kriterien:}

A. Attacken, die die Kriterien für 5.3.3 Short-lasting unilateral neuralgiform facial pain attacks (SUNFA) mit kranialen autonomen Symptomen erfüllen und periodenartig auftreten

B. Mindestens zwei Perioden von einer Dauer von 7 Tagen bis 1 Jahr, die durch schmerzfreie Remissionsphasen von $\geq 3$ Monaten voneinander getrennt sind

\subsubsection{Chronische SUNFA}

Beschreibung: SUNFA-Attacken, die für mehr als 1 Jahr ohne Remissionsphase auftreten oder mit Remissionsphasen von einer Dauer von weniger als 3 Monaten.

\section{Diagnostische Kriterien:}

A. Die Attacken erfüllen die Kriterien für 5.3.3 Short-lasting unilateral neuralgiform facial pain attacks (SUNFA) mit kranialen autonomen Symptomen sowie Kriterium B
B. Die Attacken treten mindestens 1 Jahr ohne eine Remissionsphase auf oder mit Remissionen von einer Dauer von $<3$ Monaten

\subsubsection{Hemifazialer Dauerschmerz mit autonomen Symptomen}

Zwar konnte bislang keine isolierte auf das Gesicht bezogene Entsprechung einer 3.4 Hemicrania continua wie in der ICHD-3 angegeben ermittelt werden, aber es wurde bei einer Hemicrania continua eine Schmerzausdehnung in das Gesicht beschrieben. Demzufolge können Schmerzübertragung und/oder -ausstrahlung zu Strukturen des Mund- und/oder Gesichtsbereichs diagnostische Schwierigkeiten bereiten.

Für die Hemicrania continua wurden kaum autonome Symptome beschrieben, und diese kann innerhalb der Gruppe chronischer idiopathischer Gesichtsschmerzen repräsentiert sein.

\subsection{Neurovaskuläre orofaziale Schmerzen}

Beschreibung: Mittelstarke oder starke intraorale Schmerzattacken unterschiedlicher Dauer, ohne Kopfschmerz, oft in Begleitung zahnschmerzartiger Symptome, mit leichten autonomen und/oder migränoiden Symptomen. Es finden sich zwei Unterformen:Vertreten sind Patienten mit relativ kurzen Attacken (1-4 Stunden) und solche mit längeren Attacken ( $>4$ Stunden).

\section{Diagnostische Kriterien:}

A. Mindestens fünf Attacken einseitiger intraoraler ${ }^{1}$ Schmerzen von unterschiedlicher Dauer, ohne Kopfschmerz, die die Kriterien B-D erfüllen

B. Der Schmerz weist beide der folgenden Charakteristika auf:

1. Starke oder sehr starke Intensität

2. Eine oder beide der folgenden Qualitäten:

a) Zahnschmerzartig

b) Pulsierend

C. Der Schmerz tritt in Begleitung von mindestens einem der folgenden Symptome auf:

1. Ipsilaterale Lakrimation und/oder konjunktivale Injektion

2. Ipsilateral Rhinorrhoe und/oder nasale Kongestion

3. Ipsilaterale Schwellung der Wange

4. Photophobie und/oder Phonophobie

5. Übelkeit und/oder Erbrechen

D. Der Schmerz ist nicht durch eine lokale Ursache zu erklären und die klinischen und radiologischen Befunde sind unauffällig

E. Nicht besser erklärt durch eine andere ICOP- oder ICHD-3-Diagnose

\section{Anmerkung:}

1. Obwohl es sich im Wesentlichen um intraorale Schmerzen handelt, kann es zu einer Übertragung und/oder Ausstrahlung in benachbarte Regionen kommen, vor allem bei starkem Schmerz. Dieses Phänomen bedarf einer sorgfältigen Nachverfolgung und Dokumentation. 
Kommentar: Verschiedene Untersuchungen lassen auf das Vorliegen einer spezifischen und klar abgrenzbaren Schmerzentität schließen, obwohl sie phänotypologisch einer 5.1 Orofazialen Migräne oder 5.3 Trigeminoautonomen orofazialen Schmerzen ähnelt. Obwohl diese Entität in der Fachliteratur bereits seit 1997 existiert, bedarf sie noch einer gründlichen und prospektiven Untersuchung.

Es finden sich Berichte über eine abnorme Kältesensibilität sowohl interiktal als auch während den Attacken. Dieser Befund bedarf einer gründlichen Untersuchung, da es ein nützlicher Test wäre und ein Bindeglied dieser Schmerzentität zur Migräne darstellen könnte, bei der während den Attacken eine mechanische Allodynie auftritt.

Zur Aufzeichnung aller für die Diagnosestellung erforderlichen Charakteristika ist das Führen eines Schmerztagebuchs unabdingbar.

\subsubsection{Kurz anhaltende neurovaskuläre orofaziale Schmerzen \\ Diagnostische Kriterien:}

A. Intraorale Schmerzattacken, die die Kriterien für einen 5.4 Neurovaskuläre orofaziale Schmerzen sowie Kriterium B erfüllen

B. Dauer 1-4 Stunden (unbehandelt oder bei erfolgloser Behandlung)

\subsubsection{Lang anhaltende neurovaskuläre orofaziale Schmerzen \\ Diagnostische Kriterien:}

A. Die intraoralen Schmerzattacken erfüllen die Kriterien für 5.4 Neurovaskuläre orofaziale Schmerzen sowie Kriterium B

B. Dauer $>4$ Stunden

\section{Literatur}

\subsection{Orofaziale Migräne}

Alvarez M, Montojo T, de la Casa B, et al. Unilateral nasal pain with migraine features. Cephalalgia 2013; 33: 1055-1058.

Daudia AT und Jones NS. Facial migraine in a rhinological setting. Clin Otolaryngol Allied Sci 2002; 27: 521-525.

Debruyne $\mathrm{F}$ und Herroelen L. Migraine presenting as chronic facial pain. Acta Neurol Belg 2009; 109: 235-237.

Dodick DW. Migraine with isolated facial pain: a diagnostic challenge. Cephalalgia 2007; 27: 1199-1200.

Eross E, Dodick D und Eross M. The Sinus, Allergy and Migraine Study (SAMS). Headache 2007; 47: 213-224.

Gaul C, Sandor PS, Galli U, et al. Orofacial migraine. Cephalalgia 2007; 27: 950-952.

Hussain A, Stiles MA und Oshinsky ML. Pain remapping in migraine: a novel characteristic following trigeminal nerve injury. Headache 2010; 50: 669-671.

Lipton RB, Diamond S, Reed M, et al. Migraine diagnosis and treatment: results from the American Migraine Study II. Headache 2001; 41: 638-645.

Obermann M, Mueller D, Yoon MS, et al. Migraine with isolated facial pain: a diagnostic challenge. Cephalalgia 2007; 27: 1278-1282.

Penarrocha $M$, Bandres A, Penarrocha $M$, et al. Lower-half facial migraine: a report of 11 cases. J Oral Maxillofac Surg 2004; 62: 1453-1456.

Schreiber $C P$, Hutchinson S, Webster $C$, et al. Prevalence of migraine in patients with a history of self-reported or physician-diagnosed, sinus' headache. Arch Intern Med 2004; 164: 1769-1772.

Yoon MS, Mueller D, Hansen N, et al. Prevalence of facial pain in migraine: a population-based study. Cephalalgia 2010; 30: 92-96.

\subsubsection{Orofaziale Clusterattacken}

Bahra A und Goadsby PJ. Diagnostic delays and mismanagement in cluster headache. Acta Neurol Scand 2004; 109: 175-179.

Bahra A, May A und Goadsby PJ. Cluster headache: a prospective clinical study with diagnostic implications. Neurology 2002; 58: 354-361.

Benoliel R, Birman N, Eliav E, et al. The International Classification of Headache Disorders: accurate diagnosis of orofacial pain? Cephalalgia 2008; 28: 752-762.

Benoliel R, Elishoov $\mathrm{H}$ und Sharav Y. Orofacial pain with vascular-type features. Oral Surg Oral Med Oral Pathol Oral Radiol Endod 1997; 84: 506-512.

Bittar $\mathrm{G}$ und Graff-Radford SB. A retrospective study of patients with cluster headaches. Oral Surg Oral Med Oral Pathol 1992; 73: 519-525.

Cademartiri C, Torelli P, Cologno D, et al. Upper and lower cluster headache: clinical and pathogenetic observations in 608 patients. Headache 2002; 42:630-637.

Gaul C, Gantenbein AR, Buettner UW, et al. Orofacial cluster headache. Cephalalgia 2008; 28: 903-905.

Gross SG. Dental presentations of cluster headaches. Curr Pain Headache Rep 2006; 10: 126-129.

Larner AJ. Unnecessary extractions. Br Dent J 2007; 203: 442

Sanchez Del Rio M, Leira R, Pozo-Rosich P, et al. Errors in recognition and management are still frequent in patients with cluster headache. Eur Neurol 2014; 72: 209-212.

Van Alboom E, Louis P, Van Zandijcke M, et al. Diagnostic and therapeutic trajectory of cluster headache patients in Flanders. Acta Neurol Belg 2009; 109: 10-17.

Van Vliet JA, Eekers PJ, Haan J, et al. Features involved in the diagnostic delay of cluster headache. J Neurol Neurosurg Psychiatry 2003; 74: 1123-1125.

\subsubsection{Paroxysmale hemifaziale Schmerzen}

Bahra A und Goadsby PJ. Diagnostic delays and mismanagement in cluster headache. Acta Neurol Scand 2004; 109: 175-179.

Bahra A, May A und Goadsby PJ. Cluster headache: a prospective clinical study with diagnostic implications. Neurology 2002; 58: 354-361.

Bartsch T, Knight YE und Goadsby PJ. Activation of $5-\mathrm{HT}_{1 \mathrm{~B} / 1 \mathrm{D}}$ receptor in the periaqueductal gray inhibits nociception. Ann Neurol 2004; 56: 371-381.

Benoliel R und Sharav Y. Paroxysmal hemicrania. Case studies and review of the literature. Oral Surg Oral Med Oral Pathol Oral Radiol Endod 1998; 85: 285-292.

Benoliel R, Birman N, Eliav E, et al. The International Classification of Headache Disorders: accurate diagnosis of orofacial pain? Cephalalgia 2008; 28: 752-762.

Benoliel R, Elishoov $\mathrm{H}$ und Sharav Y. Orofacial pain with vascular-type features. Oral Surg Oral Med Oral Pathol Oral Radiol Endod 1997; 84: 506-512.

Cademartiri C, Torelli P, Cologno D, et al. Upper and lower cluster headache: clinical and pathogenetic observations in 608 patients. Headache 2002; 42:630-637.

Cittadini E, Matharu MS und Goadsby PJ. Paroxysmal hemicrania: a prospective clinical study of 31 cases. Brain 2008; 131: 1142-1155.

Delcanho RE und Graff-Radford SB. Chronic paroxysmal hemicrania presenting as toothache. J Orofac Pain 1993; 7: 300-306.

Gaul C, Gantenbein AR, Buettner UW, et al. Orofacial cluster headache. Cephalalgia 2008; 28: 903-905.

Graff-Radford SB. Paroxysmal hemicrania. Oral Surg Oral Med Oral Pathol Oral Radiol Endod 1998; 86: 138.

Gross SG. Dental presentations of cluster headaches. Curr Pain Headache Rep 2006; 10: $126-129$.

Larner AJ. Unnecessary extractions. Br Dent J 2007; 203: 442.

May A. The exceptional role of the 1st division of the trigeminal nerve. Pain 2018; 159: S81-S84.

Moncada E and Graff-Radford SB. Benign indomethacin-responsive headaches presenting in the orofacial region: eight case reports. J Orofac Pain 1995; 9: 276-284.

Sanchez Del Rio M, Leira R, Pozo-Rosich P, et al. Errors in recognition and management are still frequent in patients with cluster headache. Eur Neurol 2014; 72: 209-212.

Van Alboom E, Louis P, Van Zandijcke M, et al. Diagnostic and therapeutic trajectory of cluster headache patients in Flanders. Acta Neurol Belg 2009; 109: 10-17.

Van Vliet JA, Eekers PJ, Haan J, et al. Features involved in the diagnostic delay of cluster headache. J Neurol Neurosurg Psychiatry 2003; 74: 1123-1125. 


\subsubsection{Short-lasting unilateral neuralgiform facial pain} attacks (SUNFA) mit kranialen autonomen Symptomen

Benoliel R und Sharav Y. SUNCT syndrome: case report and literature review. Oral Surg Oral Med Oral Pathol Oral Radiol Endod 1998; 85: 158-161.

Brown RS und Pass B. Orofacial pain due to trigeminal autonomic cephalgia with features of short-lasting neuralgiform headache attacks with conjunctival injection and tearing: a case report. Oral Surg Oral Med Oral Pathol Oral Radiol 2012; 114: e13-e19.

Goadsby PJ, Cittadini E und Cohen AS. Trigeminal autonomic cephalalgias: paroxysmal hemicrania, SUNCT/SUNA, and hemicrania continua. Semin Neurol 2010; 30: 186-191.

\subsubsection{Hemifazialer Dauerschmerz mit autonomen Symptomen}

Benoliel R, Robinson S, Eliav E, et al. Hemicrania continua. J Orofac Pain 2002; 16 317-325.

Goadsby PJ, Cittadini E and Cohen AS. Trigeminal autonomic cephalalgias: paroxysmal hemicrania, SUNCT/SUNA, and hemicrania continua. Semin Neurol 2010; 30: 186-191.

Hryvenko I, Cervantes-Chavarría AR, Law AS, et al. Hemicrania continua: Case series presenting in an orofacial pain clinic. Cephalalgia 2018; 38: 1950-1959.

Prakash S, Shah ND und Chavda BV. Unnecessary extractions in patients with hemicrania continua: case reports and implication for dentistry. J Orofac Pain 2010; 24: 408-411.

Rossi P, Faroni J, Tassorelli C, et al. Diagnostic delay and suboptimal management in a referral population with hemicrania continua. Headache 2009; 49: 227-234.

Viana M, Tassorelli C, Allena M, et al. Diagnostic and therapeutic errors in trigeminal autonomic cephalalgias and hemicrania continua: a systematic review. J Headache Pain 2013; 14: 14.

Ziegeler C and May A. Facial presentations of migraine, TACs, and other paroxysmal facial pain syndromes. Neurology 2019; 93: e1138-e1147.

\subsection{Neurovaskuläre orofaziale Schmerzen}

Benoliel R, Birman N, Eliav E, et al. The International Classification of Headache Disorders: accurate diagnosis of orofacial pain? Cephalalgia 2008; 28: 752-762.

Benoliel R, Elishoov $\mathrm{H}$ and Sharav Y. Orofacial pain with vascular-type features. Oral Surg Oral Med Oral Pathol Oral Radiol Endod 1997; 84: 506-512.

Czerninsky R, Benoliel R und Sharav Y. Odontalgia in vascular orofacial pain. J Orofac Pain 1999; 13: 196-200.

\section{Idiopathische orofaziale Schmerzen}

Beschreibung: Ein- oder beidseitiger intraoraler Schmerz oder Gesichtsschmerz im/in den Versorgungsgebiet/en eines Astes oder mehrerer Trigeminusäste, dessen Ätiologie unbekannt ist. Der Schmerz ist gewöhnlich anhaltend, von mittelstarker Intensität, schlecht zu lokalisieren und wird als dumpf, drückend oder brennend beschrieben.

An anderer Stelle klassifiziert: 4.1.1.3 Idiopathische Trigeminusneuralgie, 4.1.2.5 Idiopathische schmerzhafte Trigeminusneuropathie, 4.2.1.3 Idiopathische Glossopharyngeusneuralgie, 4.2.2.2 Idiopathische schmerzhafte Glossopharyngeusneuropathie.

\subsection{Syndrom des brennenden Mundes (engl. Burning mouth syndrome, BMS)}

Früher verwendete Begriffe: Stomatodynie; Glossodynie (sofern auf die Zunge beschränkt); primäres Burning-Mouth-Syndrom.
Beschreibung: Intraorale brennende Missempfindung oder Sensibilitätsstörung, die über mehr als 3 Monate für mehr als 2 Stunden/Tag täglich wiederkehrt und für die klinische Untersuchungen keine evidenten ursächlichen Läsionen ergeben.

\section{Diagnostische Kriterien:}

A. Intraorale Schmerzen, die die Kriterien B und C erfüllen

B. Täglich für $>2$ Stunden/Tag für $>3$ Monate ${ }^{1}$

C. Der Schmerz weist beide der folgenden Charakteristika auf:

1. Brennende Qualität

2. Oberflächliche Wahrnehmung in der Mundschleimhaut

D. Die Mundschleimhaut weist ein normales Erscheinungsbild auf und lokale oder systemische Ursachen wurden ausgeschlossen

E. Nicht besser erklärt durch eine andere ICOP oder ICHD-3-Diagnose ${ }^{2}$

\section{Anmerkungen:}

1. Vor Ablauf von 3 Monaten als ein 6.1.3 Wahrscheinliches Syndrom des brennenden Mundes zu kodieren, sofern alle sonstigen Kriterien erfüllt sind.

2. Die Diagnose eines 6.1 Syndroms des brennenden Mundes impliziert, dass keine quantitative sensorische Testung durchgeführt wurde. Sobald dies jedoch geschehen ist, sollte eine der beiden Unterformen, 6.1.1 Syndrom des brennenden Mundes ohne somatosensorische Veränderungen oder 6.1.2 Syndrom des brennenden Mundes mit somatosensorischen Veränderungen, diagnostiziert werden.

Kommentar: Die Ergebnisse der quantitativen sensorischen Testung sind oft auffällig (Unterscheidung zwischen den zwei Unterformen), während die klinische sensorische Untersuchung in sehr seltenen Fällen leichte sensible Defizite zeigt.

Der Schmerz bei einem 6.1 Syndrom des brennenden Mundes ist für gewöhnlich beidseitig, in seltenen Fällen jedoch einseitig. Seine Intensität fluktuiert. Die häufigste Lokalisation der Schmerzen ist in der Zungenspitze. In zwei Dritteln der berichteten Fälle treten ein Gefühl von Mundtrockenheit, Dysästhesien und ein verändertes Geschmacksempfinden auf.

Es tritt häufig bei Frauen in den Wechseljahren auf. Einige Studien zeigen psychosoziale Komorbiditäten ähnlich denen bei anderen persistierenden Schmerzerkrankungen. Neuere Daten weisen bei Patienten mit einem 6.1 Syndrom des brennenden Mundes auf unterschiedliche Ausprägungen der Somatosensorik hin. Es bleibt künftiger Forschung vorbehalten, eine mögliche Einordnung als neuropathisches Schmerzsyndrom zu treffen.

Die Symptome des Syndroms des brennenden Mundes können als sekundäre Phänomene auftreten, die auf eine lokale oder systemische Erkrankung zurückgehen, wie bei den Unterformen 1.1.3 Gingivale Schmerzen oder 1.2.1 Schmerzen in der Mundschleimhaut. Diese war zuvor unter dem Namen „sekundäres Burning-MouthSyndrom" bekannt, sie sollten jedoch als diese Erkrankungen kodiert werden. Ein 6.1 Syndrom des brennenden Mundes wird nur dann diagnostiziert, wenn alle lokalen und systemischen Ursachen ausgeschlossen wurden (früher als „primäres Burning-Mouth-Syndrom" bezeichnet). 
6.1.1 Syndrom des brennenden Mundes ohne somatosensorische Veränderungen

Beschreibung: Intraorale brennende Missempfindung oder Sensibilitätsstörung, die über mehr als 3 Monate für mehr als 2 Stunden/Tag täglich auftritt, nicht von somatosensorischen Veränderungen begleitet ist und ohne den Nachweis zugrundeliegender, die Symptomatik erklärender Läsionen in den klinischen und apparativen Untersuchungen.

\section{Diagnostische Kriterien:}

A. Orale Schmerzen, die die Kriterien für 6.1 Syndrom des brennenden Mundes erfüllen

B. Die qualitative oder quantitative somatosensorische Testung ergibt keine somatosensorischen Veränderungen.

\subsubsection{Syndrom des brennenden Mundes mit} somatosensorischen Veränderungen

Beschreibung: Intraorale brennende Missempfindung oder Sensibilitätsstörung, die über mehr als 3 Monate für mehr als 2 Stunden/Tag täglich auftritt, von negativen und/oder positiven somatosensorischen Veränderungen begleitet ist und ohne den Nachweis zugrundeliegender, die Symptomatik erklärender Läsionen in den klinischen und apparativen Untersuchungen.

\section{Diagnostische Kriterien:}

A. Intraorale Schmerzen, die die Kriterien für ein 6.1 Syndrom des brennenden Mundes erfüllen

B. Die qualitative oder quantitative somatosensorische Testung zeigt somatosensorische Veränderungen ${ }^{1}$

\section{Anmerkung:}

1. Negative (z. B. Hypästhesie und/oder Hypalgesie) und/oder positive (z. B. Hyperalgesie und/oder Allodynie) sensorische Symptome und/oder Zeichen.

\subsubsection{Wahrscheinliches Syndrom des brennenden Mundes}

Beschreibung: Intraorale brennende Missempfindung oder Sensibilitätsstörung, die für mehr als 2 Stunden/Tag täglich, jedoch über weniger als 3 Monate auftritt und ohne den Nachweis zugrundeliegender, die Symptomatik erklärender Läsionen in den klinischen und apparativen Untersuchungen.

\section{Diagnostisches Kriterium:}

A. Orale Schmerzen, die die Kriterien für ein 6.1 Syndrom des brennenden Mundes erfüllen, nur dass dieses seit $<3$ Monaten' besteht

\section{Anmerkung:}

1. Nach Ablauf von 3 Monaten, lautet die Diagnose 6.1 Syndrom des brennenden Mundes (oder eine der Unterformen).

Kommentar: Unterformen werden nicht formal klassifiziert, können jedoch gemäß den obigen Kriterien als 6.1.3.1 Wahrscheinliches Syndrom des brennenden Mundes ohne somatosensorische Verände- rungen oder 6.1.3.2 Wahrscheinliches Syndrom des brennenden Mundes mit somatosensorischen Veränderungen kodiert werden.

\subsection{Anhaltender idiopathischer Gesichtsschmerz (engl. Persistent idiopathic facial pain, PIFP)}

Früher verwendeter Begriff: Atypischer Gesichtsschmerz.

Beschreibung: Anhaltender Gesichtsschmerz mit variablem Erscheinungsbild, der mehr als 3 Monate für mehr als 2 Stunden am Tag täglich in Abwesenheit eines klinisch-neurologischen Defizits oder eines vorangegangenen ursächlichen Ereignisses auftritt.

\section{Diagnostische Kriterien:}

A. Gesichtsschmerz, der die Kriterien B und C erfüllt

B. Täglich wiederkehrend für $>2$ Stunden/Tag und für $>3$ Monate ${ }^{1}$

C. Der Schmerz weist beide der folgenden Charakteristika auf:

1. Schlecht zu lokalisieren und nicht dem Innervationsgebiet eines peripheren Nerven folgend ${ }^{2}$

2. Von dumpfer, drückender oder quälender Qualität ${ }^{3}$

D. Die klinischen und radiologischen Befunde sind unauffällig ${ }^{4}$ und lokale Ursachen wurden ausgeschlossen ${ }^{5}$

E. Nicht besser erklärt durch eine andere ICOP oder ICHD-3-Diagnose ${ }^{6}$

\section{Anmerkungen:}

1. Vor Ablauf von 3 Monaten erfolgt die Kodierung als 6.2.3 Wahrscheinlicher anhaltender idiopathischer Gesichtsschmerz, sofern alle sonstigen Kriterien erfüllt sind.

2. Der Schmerz kann als tief oder oberflächlich beschrieben werden und vom Gesicht zum Mund ausstrahlen oder umgekehrt. Im Laufe der Zeit kann es zu einer Ausbreitung in ein größeres Areal der kraniozervikalen Region kommen.

3. Zur Beschreibung des Schmerzcharakters werden vielfältige Begriffe verwendet, und der Schmerz kann Exazerbationen aufweisen und sich bei Stress verschlimmern.

4. Die klinische somatosensorische Beurteilung mit Hilfe von Pinprick (Nadelreiz) oder leichten Berührungsreizen kann in sehr seltenen Fällen leichte somatosensorische Veränderungen zeigen. Es kann noziplastischer Schmerz vorliegen, der eine veränderte somatosensorische Reizverarbeitung widerspiegelt und mit einer Veränderung der schmerzhemmenden Systeme in Verbindung steht.

5. Die Patienten können von einer kleineren Operation oder Verletzung im Gesicht, am Oberkiefer, an den Zähnen oder dem Zahnfleisch berichten, ohne dass in der klinischen oder radiologischen Untersuchung eine lokale Ursache nachweisbar ist.

6. Die quantitative sensorische Testung differenziert zwischen den beiden Unterformen. Die Diagnose 6.2 Anhaltender idiopathischer Gesichtsschmerz impliziert, dass noch keine quantitative sensorische Testung durchgeführt wurde. Ist diese erfolgt, sollte einer der beiden Subtypen, 6.2.1 Anhaltender idiopathischer Gesichtsschmerz ohne somatosensorische Veränderungen oder 6.2.2 Anhaltender idiopathischer Gesichts- 
schmerz mit somatosensorischen Veränderungen diagnostiziert werden.

Kommentar: In der ICHD-3 werden zwei Störungen als ein und dieselbe Schmerzentität behandelt: 13.12 Anhaltender idiopathischer Gesichtsschmerz, mit atypischer Odontalgie als möglicher Unterform. In den vorliegenden ICOP-Kriterien werden zwei Entitäten unterschieden und definiert: 6.2 Anhaltender idiopathischer Gesichtsschmerz und 6.3 Anhaltende idiopathische dentoalveoläre Schmerzen. Diese Störungen lösen Gesichtsschmerz beziehungsweise dentoalveoläre Schmerzen aus, die in beiden Fällen einen relativ konstanten Verlauf aufweisen, aber nichtsdestotrotz zurVerschlimmerung neigen.

6.2 Anhaltender idiopathischer Gesichtsschmerz kann als Komorbidität mit anderen Schmerzerkrankungen wie Fibromyalgiesyndrom (engl.: ",chronic widespread pain",CWP) und Reizdarmsyndrom aufweisen. Darüber hinaus kann er mit psychosozialen Komorbiditäten ähnlich denen bei anderen anhaltenden Schmerzerkrankungen auftreten.

6.2.1 Anhaltender idiopathischer Gesichtsschmerz ohne somatosensorische Veränderungen

Beschreibung: Anhaltender Gesichtsschmerz mit variablen Eigenschaften, der mehr als 3 Monate lang täglich für mehr als 2 Stunden am Tag auftritt, nicht begleitet von somatosensorischen Veränderungen und ohne klinisch neurologisches Defizit oder vorangegangenes ursächliches Ereignis.

\section{Diagnostische Kriterien:}

A. Gesichtsschmerz, der die Kriterien für einen 6.2 Anhaltenden idiopathischen Gesichtsschmerz erfüllt

B. Die qualitative oder quantitative somatosensorische Testung ergibt keine somatosensorischen Veränderungen

\subsubsection{Anhaltender idiopathischer Gesichtsschmerz mit} somatosensorischen Veränderungen

Beschreibung: Anhaltender Gesichtsschmerz mit variablen Eigenschaften, der mehr als 3 Monate lang täglich für mehr als 2 Stunden am Tag auftritt und von negativen und/oder positiven somatosensorischen Veränderungen begleitet wird, ohne klinisch neurologisches Defizit oder vorangegangenes ursächliches Ereignis.

\section{Diagnostische Kriterien:}

A. Gesichtsschmerz, der die Kriterien für 6.2 Anhaltender idiopathischer Gesichtsschmerz erfüllt

B. Die qualitative oder quantitative somatosensorische Testung ergibt somatosensorische Veränderungen ${ }^{1}$

\section{Anmerkung:}

1. Negative (d.h. Hypästhesie und/oder Hypalgesie) und/oder positive (d. h. Hyperalgesie und/oder Allodynie) sensorische Symptome und/oder Zeichen.
6.2.3 Wahrscheinlicher anhaltender idiopathischer Gesichtsschmerz

Beschreibung: Gesichtsschmerz mit variablen Eigenschaften, der für mehr als 2 Stunden am Tag auftritt, jedoch weniger als 3 Monate lang, ohne klinisches neurologisches Defizit oder vorangegangenes ursächliches Ereignis.

\section{Diagnostisches Kriterium:}

A. Gesichtsschmerz, der die Kriterien für 6.2 Anhaltender idiopathischer Gesichtsschmerz erfüllt, nur dass er seit $<3$ Monaten ${ }^{1}$ vorliegt

\section{Anmerkung:}

1. Nach Ablauf von 3 Monaten wird ein 6.2 Anhaltender idiopathischer Gesichtsschmerz (oder einer seiner Subtypen) diagnostiziert.

Kommentar: Die Unterformen werden nicht formal klassifiziert, können aber nach den obigen Kriterien als 6.2.3.1 Wahrscheinlicher anhaltender idiopathischer Gesichtsschmerz ohne somatosensorische Veränderungen oder 6.2.3.2 Wahrscheinlicher anhaltender idiopathischer Gesichtsschmerz mit somatosensorischen Veränderungen kodiert werden.

\subsection{Anhaltende idiopathische dentoalveoläre} Schmerzen

Früher verwendete Begriffe: Atypische Odontalgie; primäre persistierende dentoalveoläre Schmerzstörung (PDAP); Phantomzahnschmerz.

Beschreibung: Anhaltende einseitige intraorale dentoalveoläre Schmerzen, selten an mehreren Stellen auftretend, mit variablen Eigenschaften, jedoch mehr als 3 Monate lang täglich für mehr als 2 Stunden wiederkehrend, ohne vorangegangenes ursächliches Ereignis.

\section{Diagnostische Kriterien:}

A. Intraorale dentoalveoläre Schmerzen, die die Kriterien B und C erfüllen

B. Täglich für $>2$ Stunden/Tag $>3$ Monate lang wiederkehrend ${ }^{1}$

C. Der Schmerz weist beide der folgenden Charakteristika auf: 1. Dentoalveolär lokalisiert (Zahn oder Alveolarknochen) ${ }^{2}$

2. Tiefe, dumpfe, drückende Qualität ${ }^{3}$

D. Die klinischen und radiologischen Untersuchungsbefunde sind unauffällig ${ }^{4}$ und lokale Ursachen wurden ausgeschlossen

E. Nicht besser erklärt durch eine andere ICOP oder ICHD-3-Diagnose ${ }^{5}$

\section{Anmerkungen:}

1. Vor Ablauf von 3 Monaten erfolgt, sofern alle sonstigen Kriterien erfüllt sind, eine Kodierung als 6.3.3 Wahrscheinliche anhaltende idiopathische dentoalveoläre Schmerzen.

2. Der Schmerz findet sich selten an mehreren Stellen. Im Laufe der Zeit kann es zu einer Ausbreitung in ein größeres Areal der kraniozervikalen Region kommen. 
3. Es wird eine Vielzahl von Begriffen verwendet, um den Charakter und die Qualität des Schmerzes zu beschreiben. Er kann als entweder tief oder oberflächlich beschrieben werden, und es kann eine zusätzliche Symptombeschreibung hinzukommen, um die Komplexität der mit dieser Störung verbundenen Wahrnehmungen zu beschreiben. Darüber hinaus kann der Schmerz Exazerbationen aufweisen und sich durch Stress verschlimmern.

4. Die klinische somatosensorische Beurteilung mit Hilfe von Pinprick/Nadelreizen oder leichten Berührungsreizen zeigt nur in sehr seltenen Fällen sensible Veränderungen. Es kann ein noziplastischer Schmerz vorliegen, der eine veränderte Reizverarbeitung des somatosensorischen Systems widerspiegelt und mit einer Veränderung der Schmerzhemmung in einhergeht. Die quantitative sensorische Testung ergibt eine Differenzierung zwischen den beiden Unterformen. Die Diagnose 6.3 Anhaltende idiopathische dentoalveoläre Schmerzen impliziert, dass noch keine quantitative sensorische Testung durchgeführt wurde. Ist diese erfolgt, sollte einer der beiden Subtypen, 6.3.1 Anhaltende idiopathische dentoalveoläre Schmerzen ohne somatosensorische Veränderungen oder 6.3.2 Anhaltende idiopathische dentoalveoläre Schmerzen mit somatosensorischen Veränderungen diagnostiziert werden.

Kommentar: In der ICHD-3 werden zwei Störungen als ein und dieselbe Schmerzentität behandelt: 13.12 Anhaltender idiopathischer Gesichtsschmerz, mit atypischer Odontalgie als möglicher Unterform. In den vorliegenden ICOP-Kriterien werden zwei Entitäten definiert und unterschieden: 6.2 Anhaltender idiopathischer Gesichtsschmerz und 6.3 Anhaltende idiopathische dentoalveoläre Schmerzen. Diese Störungen lösen Gesichtsschmerz beziehungsweise dentoalveoläre Schmerzen aus, die in beiden Fällen einen relativ konstanten Verlauf haben, aber nichtsdestotrotz zur Verschlimmerung neigen.

\subsubsection{Anhaltende idiopathische dentoalveoläre}

Schmerzen ohne somatosensorische Veränderungen

Beschreibung: Anhaltende einseitige intraorale dentoalveoläre Schmerzen, selten an mehreren Stellen auftretend, mit variablen Eigenschaften, mehr als 3 Monate lang täglich für mehr als 2 Stunden wiederkehrend, nicht begleitet von somatosensorischen Veränderungen bei Nichtvorliegen eines vorangegangenen ursächlichen Ereignisses.

\section{Diagnostische Kriterien:}

A. Intraorale Schmerzen, die die Kriterien für 6.3 Anhaltende idiopathische dentoalveoläre Schmerzen erfüllen

B. Die qualitative oder quantitative somatosensorische Testung ergibt keine somatosensorischen Veränderungen

\subsubsection{Anhaltende idiopathische dentoalveoläre} Schmerzen mit somatosensorischen Veränderungen

Beschreibung: Anhaltende einseitige intraorale dentoalveoläre Schmerzen, selten an mehreren Stellen auftretend, mit variablen Eigenschaften, jedoch mehr als 3 Monate lang täglich für mehr als 2 Stunden wiederkehrend und begleitet von negativen und/oder positiven somatosensorischen Veränderungen, bei Fehlen eines vorangegangenen ursächlichen Ereignisses.

\section{Diagnostische Kriterien:}

A. Intraorale Schmerzen, die die Kriterien für 6.3 Anhaltende idiopathische dentoalveoläre Schmerzen erfüllen

B. Die qualitative oder quantitative somatosensorische Testung ergibt somatosensorische Veränderungen ${ }^{1}$

\section{Anmerkung:}

1. Es liegen negative (d.h. Hypästhesie und/oder Hypalgesie) und/oder positive (d. h. Hyperalgesie und/oder Allodynie) sensorische Symptome und/oder Zeichen vor, aber nicht räumlich begrenzt auf ein neuroanatomisch relevantes Areal, im Gegensatz zu einer 4.1.2.3 Posttraumatischen schmerzhaften Trigeminusneuropathie.

\subsubsection{Wahrscheinliche anhaltende idiopathische} dentoalveoläre Schmerzen

Beschreibung: Einseitige intraorale dentoalveoläre Schmerzen, selten an mehreren Stellen auftretend, mit variablen Eigenschaften, der täglich für mehr als 2 Stunden auftritt, dies jedoch weniger als 3 Monate lang, bei Fehlen eines vorangegangenen ursächlichen Ereignisses.

\section{Diagnostisches Kriterium:}

A. Intraorale Schmerzen, die die Kriterien für 6.3 Anhaltende idiopathische dentoalveoläre Schmerzen erfüllen, außer dass sie seit $<3$ Monaten ${ }^{1}$ vorliegen

\section{Anmerkung:}

1. Nach Ablauf von 3 Monaten lautet die Diagnose 6.3 Anhaltende idiopathische dentoalveoläre Schmerzen (oder ein Subtyp hiervon).

Kommentar: Unterformen werden nicht formal klassifiziert, können jedoch nach den obigen Kriterien als 6.3.3.1 Wahrscheinliche anhaltende idiopathische dentoalveoläre Schmerzen ohne somatosensorische Veränderungen oder 6.3.3.2 Wahrscheinliche anhaltende idiopathische dentoalveoläre Schmerzen mit somatosensorischen Veränderungen kodiert werden.

\subsection{Constant unilateral facial pain with additional attacks (CUFPA)}

Beschreibung: Dumpfer einseitiger dauerhafter Gesichtsschmerz (ohne Remissionsphasen) von leichter bis mittelstarker Intensität, begleitet von klar umrissenen mittelstarken bis starken Schmerzattacken im gleichen Areal von 10-30 Minuten Dauer. Weder der Dauerschmerz noch die zusätzlichen Schmerzattacken werden von typischen autonomen und/oder migräneartigen Merkmalen begleitet.

\section{Diagnostische Kriterien:}

A. Streng einseitiger dauerhafter Gesichtsschmerz, der Kriterium B erfüllt, mit Exazerbationen, die Kriterium C erfüllen 
B. Hintergrundschmerz, der beide der folgenden Charakteristika aufweist:

1. Leichte bis mittelstarke Intensität

2. Für $>3$ Monate dauerhaft vorliegend

C. Exazerbationen, die als klar umrissene Schmerzattacken auftreten, bis zu sechsmal am Tag, mit allen der folgenden drei Charakteristika:

1. Im gleichen Areal lokalisiert wie der Hintergrundschmerz

2. Mittelstarke bis starke Intensität

3. 10-30 Minuten Dauer

D. Die klinischen und radiologischen Befunde sind unauffällig und lokale Ursachen wurden ausgeschlossen

E. Nicht besser erklärt durch eine andere ICOP oder ICHD-3-Diagnose ${ }^{1}$

\section{Anmerkung:}

1. Die anfallsartigen Exazerbationen müssen in Form von Schmerzattacken auftreten, die sich eindeutig vom Hintergrundschmerz abheben, wobei die Patienten beschreiben, dass die Schmerzen die hier umrissenen zwei Kombinationen von Merkmalen aufweisen; ansonsten sollten die Diagnose von 5.3.2 Paroxysmalen hemifazialen Schmerzen oder eines 6.2 Anhaltenden idiopathischen Gesichtsschmerzes erwogen werden. Ein Ansprechen auf Indometacin sollte eher zu der Diagnose der 5.3.2 Paroxysmalen hemifazialen Schmerzen führen.

Kommentar: Autonome Symptome können fehlen, schließen aber nicht 6.4 Constant unilateral facial pain with additional attacks aus.

Derzeit liegen keine ausreichenden Daten vor, um episodische und chronische Unterformen eines 6.4 Constant unilateral facial pain with additional attacks vorzuschlagen.

\section{Literatur}

Baad-Hansen L. Atypical odontalgia - pathophysiology and clinical management. JOral Rehabil 2008; 35: 1-11.

Baad-Hansen L, Leijon G, Svensson P, et al. Comparison of clinical findings and psychosocial factors in patients with atypical odontalgia and temporomandibular disorders. J Orofac Pain 2008; 22: 7-14.

Baad-Hansen L, Pigg M, Ivanovic SE, et al. Intraoral somatosensory abnormalities in patients with atypical odontalgia - a controlled multicenter quantitative sensory testing study. Pain 2013; 154: 1287-1294.

Benoliel R und Gaul C. Persistent idiopathic facial pain. Cephalalgia 2017; 37 : 680-691.

Durham J, Exley C, John MT, et al. Persistent dentoalveolar pain: the patient's experience. J Orofac Pain 2013; 27: 6-13.

Forssell H, Jääskeläinen S, List T, et al. An update on pathophysiological mechanisms related to idiopathic oro-facial pain conditions with implications for management. J Oral Rehabil 2015; 42: 300-322.

Forssell $\mathrm{H}$, Tenovuo O, Silvoniemi P, et al. Differences and similarities between atypical facial pain and trigeminal neuropathic pain. Neurology 2007; 69:1451-1459.

Grémeau-Richard C, Dubray C, Aublet-Cuvelier B, et al. Effect of lingual nerve block on Burning-Mouth-Syndrom (stomatodynia): a randomized crossover trial. Pain 2010; 149: 27-32.

Hagelberg N, Jääskeläinen SK, Martikainen IK, et al. Striatal dopamine D2 receptors in modulation of pain in humans: a review. Eur J Pharmacol 2004; 500: 187-192.

Häggman-Henrikson B, Alstergren P, Davidson T, et al. Pharmacological treatment of oro-facial pain - health technology assessment including a systematic review with network meta-analysis. J Oral Rehabil 2017; 44: 800-826.

Jääskeläinen SK. Pathophysiology of primary burning mouth syndrome. Clin Neurophysiol 2012; 123: 71-77.
Jääskeläinen SK und Woda A. Burning mouth syndrome. Cephalalgia 2017; 37: 627-647.

Kolkka-Palomaa M, Jääskeläinen SK, Laine MA, et al. Pathophysiology of primary burning mouth syndrome with special focus on taste dysfunction: a review. Oral Dis 2015; 21: 937-948.

Lang E, Kaltenhäuser M, Seidler S, et al. Persistent idiopathic facial pain exists independent of somatosensory input from the painful region: findings from quantitative sensory functions and somatotopy of the primary somatosensory cortex. Pain 2005; 118: 80-91.

List T, Leijon G und Svensson P. Somatosensory abnormalities in atypical odontalgia: A case-control study. Pain 2008; 139: 333-341.

McMillan R, Forssell H, Buchanan JA, et al. Interventions for treating burning mouth syndrome. Cochrane Database Syst Rev 2016; 11: CD002779.

Nixdorf DR, Drangsholt MT, Ettlin DA, et al. Classifying orofacial pains: a new proposal of taxonomy based on ontology. J Oral Rehabil 2012; 39: 161-169.

Puhakka A, Forssell H, Soinila S, et al. Peripheral nervous system involvement in primary burning mouth syndrome - results of a pilot study. Oral Dis 2016; 22: 338-344.

Scala A, Checchi L, Montevecchi M, et al. Update on burning mouth syndrome: overview and patient management. Crit Rev Oral Biol Med 2003; 14: 275-291.

Ziegeler $C$ und May A. Facial presentations of migraine, TACs, and other paroxysmal facial pain syndromes. Neurology 2019; 93: e1138-e1147.

\section{Psychosoziale Beurteilung von Patienten mit orofazialen Schmerzen}

\section{Einführung}

Das biopsychosoziale Modell berücksichtigt neben biologischen auch psychische und soziale Faktoren. Als Ergebnis sollen somatische Erkrankungen in ihrem Verlauf und hinsichtlich psychosozialer Begleitumstände umfassender verstanden und behandelt werden. Die wichtigsten psychischen Faktoren, die bei Schmerzerkrankungen relevant sind, umfassen Angst, Depression, subjektive körperliche Symptome, Katastrophisieren sowie Vermeidungsverhalten. Die wichtigsten sozialen Faktoren umfassen den Zugang zu medizinischer Versorgung, das Ausmaß an Stigmatisierung und die verfügbare Unterstützung aus dem Familien- und Freundeskreis. Für jeden dieser Faktoren gibt es umfangreiche empirische Belege für ihren Zusammenhang mit Schmerzerkrankungen, und die Daten belegen die zentrale Bedeutung des biopsychosozialen Modells für das Verständnis der Komplexität der Schmerzverarbeitung im Allgemeinen aber auch [1] in Bezug auf orofaziale Schmerzerkrankungen [2-6]. Dabei wird die Implementierung des biopsychosozialen Modells in der Forschung und der klinischen Schmerzmedizin unterschiedlich gehandhabt [7-9]. Neue Taxonomien für chronische Schmerzerkrankungen betonen eindeutig die zentrale Bedeutung sowohl körperlicher Faktoren für die Erkrankungen als auch die zusätzliche Berücksichtigung psychosozialer Faktoren [10].

Für das Gebiet der orofazialen Schmerzen werden hier Empfehlungen zu einer optimalen Forschungspraxis skizziert, die im Einklang mit den Absichten der ICOP-Taxonomie stehen und sich an die kürzlich etablierten Research Diagnostic Criteria for Temporomandibular Disorders (RDC/TMD) [11] und an die in den Diagnostic Criteria for TMD (DC/TMD) [12, 13] festgeschriebenen Empfehlungen halten. Dort werden die relevanten Konstrukte und angemessene Instrumente für die Beurteilung von muskuloskeletalen Schmerzen (z. B. schmerzhafte temporomandibuläre Dysfunktionen [TMD]) genannt. Zwar stammen diese Empfehlungen aus Forschungsergebnissen zu den temporomandibulären Dysfunktionen, einer Untergruppe der orofazialen Schmerzen, doch 


\begin{tabular}{|c|c|c|c|c|}
\hline Instrument & $\begin{array}{l}\text { Anz. d. } \\
\text { Fragen/Items }\end{array}$ & $\begin{array}{l}\text { Umfassende } \\
\text { Beurteilung }\end{array}$ & $\begin{array}{l}\text { Standard- } \\
\text { Screening }\end{array}$ & $\begin{array}{l}\text { Kurz- } \\
\text { Screening }\end{array}$ \\
\hline $\begin{array}{l}\text { Schmerz- } \\
\text { zeichnung }\end{array}$ & 1 & + & + & + \\
\hline GCPS v2.0 & 8 & + & + & + \\
\hline $\begin{array}{l}\text { JFLS (Lang- } \\
\text { form) }\end{array}$ & 20 & + & & \\
\hline $\begin{array}{l}\text { JFLS (Kurz- } \\
\text { form) }\end{array}$ & 8 & & + & \\
\hline PHQ-4 & $5^{\mathrm{a}}$ & & + & + \\
\hline PHQ-9 & $10^{\mathrm{a}}$ & + & & \\
\hline GAD-7 & $8^{\mathrm{a}}$ & + & & \\
\hline PHQ-15 & 15 & + & & \\
\hline $\mathrm{OBC}$ & 20 & + & + & \\
\hline \multicolumn{5}{|c|}{$\begin{array}{l}\text { GAD Generalized Anxiety Disorder (generalisierte Angststörung), GCPS Graded } \\
\text { Chronic Pain Scale (Skala zur Graduierung chronischer Schmerzen), JFLS } \\
\text { Jaw Functional Limitation Scale (Skala zu funktionellen Beeinträchtigungen } \\
\text { des Kiefers, PHQ Patient Health Questionnaire (Gesundheitsfragebogen für } \\
\text { Patienten), OBC Oral Behaviours Checklist (Checkliste von auf den Mund- und } \\
\text { Kieferbereich bezogenen Verhaltensweisen). } \\
\text { aDie Anzahl der Punkte beinhaltet eine Reflexionsfrage zur funktionellen } \\
\text { Auswirkung berichteter Symptome. }\end{array}$} \\
\hline
\end{tabular}

gibt es derzeit keine Hinweise auf Unterschiede zwischen orofazialen Schmerzerkrankungen, die nicht mit temporomandibulären Dysfunktionen zusammenhängen, und denen, die mit temporomandibulären Dysfunktionen einhergehen. Dementsprechend sollte sich die Forschungsarbeit in diesem Stadium der Entwicklung der ICOP gleichermaßen auch mit dem biopsychosozialen Modell und den relevanten psychosozialen Konstrukten beschäftigen. Sich hieraus ergebende strukturierte und systematische Befunde werden ein stärker empirisch gestütztes Beurteilungsmodell für nicht auf temporomandibuläre Dysfunktionen zurückgehende orofaziale Schmerzen erlauben und zu einer Korrektur dieser initialen Empfehlungen führen.

\section{Ebenen der psychosozialen Beurteilung}

Von der DC/TMD [13) werden zwei Ebenen der psychosozialen Beurteilung definiert, mit einer weiteren wurde einer spezifischen Anfrage aus der klinischen Praxis Rechnung getragen (Zusammenfassung siehe Tab. 1). Die Kurz-Screening-Version ist für Forschungs- (und klinische) Settings gedacht, in die sich nur eine sehr knappe biopsychosoziale Beurteilung - mit einer geringstmöglichen Anzahl von Fragen - integrieren lässt [14]. Interessanterweise wurden die gleichen Komponenten dieses kurzen Screenings informell auch von anderen Kollegen beschrieben, was auf eine Konvergenz auf ein paar wenige zu bewertende psychosoziale Kernbereiche hinweist. In die Standard-Screening-Version wurden zwei weitere Instrumente aufgenommen. Man sollte sich darüber im Klaren sein, dass diese beiden Formen des Screenings sehr begrenzt sind.

Die umfassende Beurteilung richtet sich gezielt an klinische Forscher. Sie soll diese in die Lage versetzen, alle relevanten Konstrukte reliabel zu erfassen und damit eine bessere Charakterisierung ihrer Patientenstichproben hinsichtlich eines psychosozialen Profils zu ermöglichen. Sämtliche Instrumente und Interpretationshilfen sind auf der Website www.rdc-tmdinternational.org frei verfügbar. Darüber hinaus werden unten einige weitere Instrumente empfohlen, deren Einsatz in Betracht zu ziehen ist.

\section{Schmerz- und funktionsbezogene Konstrukte und Instrumente für orofaziale Schmerzen}

Schmerzausmaß. Die Schmerzzeichnung (Einzeichnung schmerzender Stellen in ein Schema des menschlichen Körpers) bietet eine einfache Erfassung aller Schmerzlokalisation(en), wobei multiple Lokalisationen ein Risikofaktor für die Chronifizierung von Schmerzen sind [15]. Sämtliche Schmerzerkrankungen - unabhängig vom mutmaßlichen nozizeptiven Mechanismus - scheinen im Hinblick auf das Schmerzausmaß ähnlich betroffen.

Schmerzintensität und schmerzbedingte Einschränkung. Die Graded Chronic Pain Scale (GCPS, v2.0) ist ein weitverbreitetes und validiertes Instrument zur Prüfung der Schmerzdauer, der Schmerzintensität und der schmerzbedingten Aktivitätseinschränkung. Sie wird auch als Graded Chronic Pain Status bezeichnet und kann eingesetzt werden, um Patienten hinsichtlich des erforderlichen Behandlungsumfanges einzuteilen [16-18]. Der GCPS ist zudem ein prognostischer Indikator. Je höher der Wert des GCPS, desto höher ist das Risiko für Schmerzchronifizierung [19].

Funktionelle Einschränkung. Die von einer Person erfahrene Beeinträchtigung funktioneller Fähigkeiten wird als, funktionelle Einschränkung" bezeichnet [20]. Die Jaw Functional Limitation Scale (JFLS) existiert in zwei Versionen: a) eine Version mit acht Items, die ein allgemeines Bild ergibt, und b) eine Version mit 20 Items, die drei Bereiche erfasst (Einschränkung beim Kauen, bei der Kieferöffnung sowie beim verbalen und emotionalen Ausdruck) [21, 22]. Beide Versionen sind gleichermaßen reliabel und valide, darüber hinaus sind sie veränderungssensitiv. Während eine funktionelle Einschränkung bei muskuloskeletalen Schmerzen wesentlich ist (und damit ihre Relevanz für TMDs offensichtlich), wird bei orofazialen Schmerzen, die nicht auf eine TMD zurückzuführen sind, davon ausgegangen, dass ihre funktionellen Konsequenzen eine Reaktion auf diese Schmerzen sind. Über die Ursachen dieser funktionellen Konsequenzen wird gemutmaßt [22], allerdings müssen sie weiter erforscht werden, um die vollständige Bedeutung von orofazialen Schmerzen zu verstehen.

Zu Überbeanspruchung führende Verhaltensweisen. Die Oral Behaviours Checklist (OBC) umfasst eine Auflistung von 21 Aktivitäten im orofazialen Bereich, wie Zähne zusammenzubeißen, Anspannen des Unterkiefers oder Sprechen. Die Checkliste verfügt über gute psychometrische Eigenschaften [23-25] und die OBC-Werte zeigen einen Zusammenhang mit TMD [15, 26-30]. Ob diese Verhaltensweisen spezifisch für orofaziale Schmerzen sind, bleibt offen. Es ist bekannt, dass Schonverhalten Einfluss auf nichtmuskuloskeletale Rückenschmerzen hat [31], was eine Anwendbarkeit auf orofaziale Schmerzen, die nicht auf eine TMD zurückgehen, nahelegt. 
Psychosoziale Konstrukte und Instrumente im

Zusammenhang mit orofazialen Schmerzen

Depression und Angst. Das PRIME-MD-Projekt (PRIMary care Evaluation of Mental Disorders) [32] fußte zunächst auf psychischen Störungen und zielte darauf ab, psychosoziale Instrumente für die Beurteilung von fünf der häufigsten psychischen Probleme zu entwickeln, die sich Hausärzten präsentieren: Angst, Depression, somatoforme, alkoholbezogene und Essstörungen [33]. Besonders relevant für Schmerzerkrankungen sind der neun Fragen umfassende Gesundheitsfragebogen für Patienten (PHQ-9) zu Depression und die aus sieben Fragen bestehende Generalized Anxiety Disorder Scale (GAD-7) zur Erfassung generalisierter Angst. Beide Instrumente erlauben eine reliable und valide Messung der entsprechenden Konstrukte. Jedes Instrument steuert zwei Fragen zu dem kurzen PHQ-4 Screening-Instrument zu Depression und Angst bei, das zur Beurteilung der psychischen Belastung (engl.: "distress") genutzt wird und in Nordamerika und Europa weithin angewendet wird. Depression (gemessen mit PHQ-9, PHQ-4) ist eine Stimmungslage, die durch anhaltende Schmerzen beeinflusst wird und selbst Einfluss auf die Schmerzverarbeitung hat. Sie scheint für orofaziale Schmerzen von besonderer Relevanz zu sein [34]. Angst (gemessen mit GAD-7, PHQ-4) manifestiert sich oft als Sorge und generalisierte Aktivierung des sympathischen Nervensystems, sie steht mit Schmerzwahrnehmung [35] und Hypervigilanz [36] in Verbindung. Angst ist im medizinischen Setting allgegenwärtig und scheint bei orofazialen Schmerzen von besonderer Relevanz zu sein [37].

Somatoforme Störungen. Aus dem PRIME-MD-Projekt entstammt auch der Patient Health Questionnaire-15 (Gesundheitsfragebogen für Patienten PHQ-15), ein Patientenfragebogen zur Erfassung der Schwere somatischer Symptome. Körperliche Symptome, die nicht im Rahmen einer Krankheitsdiagnose auftreten, stellen in allen Disziplinen der Medizin eine besondere Herausforderung dar. Befunde dieser Art werden oft als somatoforme Störung, funktionelle Störungen, medizinisch unerklärliche Symptome und Symptome ohne Krankheitswert beschrieben [38, 39]. Keiner dieser Begriffe ist jedoch ausreichend für die Beschreibung von etwas, das ein komplexeres Konstrukt darstellt als ursprünglich angenommen. Zwar werden diverse Mechanismen postuliert, die den berichteten körperlichen Symptomen zugrunde liegen könnten [39-41], sie alle decken sich jedoch auch mit den vielfältigen Begleitsymptomen anhaltender orofazialer Schmerzen [37, 42]. Eine erweiterte Betrachtung des Phänomens bezieht auch die okklusale Dysästhesie [43] mit ein, die potenziell für wenigstens einige der orofazialen Schmerzen innerhalb der ICOP von Belang ist.

Katastrophisieren. Katastrophisieren bzgl. Schmerzen ist die Neigung, den Bedrohungswert des Schmerzreizes aufzubauschen und sich im Kontext von Schmerzen hilflos zu fühlen. Hinzu kommt die relative Unfähigkeit, seine um die Schmerzen kreisenden Gedanken in Erwartung eines, während einem oder nach einem schmerzvollen Ereignis zu unterdrücken [44]. Ausgeprägteres Katastrophisieren geht mit einer erhöhten Inanspruchnahme von medizinischer Versorgung, einer erhöhten Schmerzexpression und schlechteren
Behandlungsergebnissen einher [45-47]. Katastrophisieren ist kein Standardmaß im Rahmen von DC/TMD, da die Evidenz für die Relevanz des Katastrophisierens bzgl. TMD zu dem Zeitpunkt, als die Empfehlungen der DC/TMD-Achse II formuliert wurden, nicht ausreichte. Die Situation hat sich seitdem verändert - und darüber hinaus ist es mit Blick auf seine Relevanz für Schmerzerkrankungen nun angemessen, dieses Konstrukt als entsprechendes Maß in die ICOP aufzunehmen. Zu den entsprechenden validierten Messinstrumenten gehören die Pain Catastrophizing Scale [48] und der Coping Strategies Questionnaire [49].

Angstvermeidung (Fear Avoidance). Das Angstvermeidungsmodell ist aus operanten Modellen zu Rückenschmerzen hervorgegangen [50], basierend auf Verhaltensbeobachtungen, bei denen von Schmerzen berichtet wurde, die sich nicht mit körperlichen Befunden deckten. Das Modell der Fear Avoidance wurde seitdem durch umfassende Untersuchungen untermauert [51]. In dem Modell führt fehlende Angst vor verletzungsbedingten de novo einsetzenden Schmerzen zu einem angemessenen Verhalten, das eine Genesung erlaubt. Umgekehrt führt Angst vor dem Schmerz zum Katastrophisieren, zur Vermeidung von möglicherweise schmerzauslösenden Situationen und einer dementsprechenden Schonung der relevanten Körperteile, in der Folge treten Depression und Beeinträchtigung auf. Die Beeinträchtigungen nähren dann wiederum das Schmerzerleben. Sie leisten einem Vermeidungsverhalten Vorschub und sorgen für eine ausbleibende Genesung. Dieses Modell ist für motorisches Verhalten eindeutig relevant, und langsam zeigen sich auch Befunde, die eine Relevanz dieses Konstrukts für TMD nahelegen. Allerdings handelt es sich um ein Modell auf der Ebene der einzelnen Person. Es geht um die Auswirkungen von Verhaltens- und Denkweisen auf das Zentralnervensystem; vor diesem Hintergrund wird davon ausgegangen, dass eine Verbindung zur Schmerzverarbeitung besteht. Die eindeutig plausiblen Hypothesen, die bei Menschen mit Verletzungen des Kauapparats eine Verbindung zwischen der Angst vor Bewegungen und der Genesung herstellen und die ein wahrscheinliches Auftreten chronischer Schmerzen bei einigen dieser Menschen nahelegen, bedürfen dementsprechend noch näherer Untersuchungen [52]. Die aktuelle Datenlage lässt darauf schließen, dass diese Perspektive auch für orofaziale Schmerzen gilt.

Angst vor Schmerzen wird anhand verschiedener Instrumente gemessen, von denen das bekannteste die Tampa Scale for Kinesiophobia (TSK) [53] ist, welche sehr nutzbringend bei Patienten mit Rückenschmerzen eingesetzt worden ist [54]. Die TSK wurde für den Kauapparat entsprechend angepasst. Die TSK-TMD [55] scheint sowohl das somatische Erleben als auch vermeidende Verhaltensweisen zu erfassen, die orofazialen Schmerzen vorangehen können.

\section{Schlussfolgerungen und Zukunftsperspektive}

Es besteht eindeutig Bedarf an weiteren Untersuchungen zum biopsychosozialen Modell und dessen klinischer wie auch forschungsbezogener Relevanz für orofaziale Schmerzen. Mit der Weiterentwicklung und feineren Ausarbeitung der Kriterien für die in der ICOP beschriebenen Erkrankungen sollte es zu einem 
ähnlichen Fortschritt im Hinblick auf unser Verständnis der von diesen Schmerzerkrankungen betroffenen Personen kommen. Wenn sich Taxonomie und Diagnosestellung verbessern, sollten daraus sowohl ein besseres Verständnis der Schmerzmechanismen als auch fundierte Behandlungsempfehlungen resultieren. Es werden vor allem multimodale Ansätze gefragt sein, um den potenziellen therapeutischen Gewinn standardmäßiger biomedizinischer Therapie, wie pharmakologische oder chirurgischen Ansätze, zu erhöhen [56]. Derzeit empfiehlt sich dringend ein konsequenter Einsatz einer standardisierten psychosozialen Beurteilung der Patienten mit orofazialen Schmerzen, parallel zur Verwendung der dargelegten Kriterien (und ihrer Erweiterungen zu Forschungszwecken) für die in der ICOP zusammengefassten Erkrankungen.

\section{Danksagung. Dieses Kapitel ist eine Adaptation von Ohrbach und Durham} [8].

Open Access. Dieser Artikel wird unter der Creative Commons Namensnennung 4.0 International Lizenz veröffentlicht, welche die Nutzung, Vervielfältigung, Bearbeitung, Verbreitung und Wiedergabe in jeglichem Medium und Format erlaubt, sofern Sie den/die ursprünglichen Autor(en) und die Quelle ordnungsgemäß nennen, einen Link zur Creative Commons Lizenz beifügen und angeben, ob Änderungen vorgenommen wurden.

Die in diesem Artikel enthaltenen Bilder und sonstiges Drittmaterial unterliegen ebenfalls der genannten Creative Commons Lizenz, sofern sich aus der Abbildungslegende nichts anderes ergibt. Sofern das betreffende Material nicht unter der genannten Creative Commons Lizenz steht und die betreffende Handlung nicht nach gesetzlichen Vorschriften erlaubt ist, ist für die oben aufgeführten Weiterverwendungen des Materials die Einwilligung des jeweiligen Rechteinhabers einzuholen.

Weitere Details zur Lizenz entnehmen Sie bitte der Lizenzinformation auf http:// creativecommons.org/licenses/by/4.0/deed.de.

\section{Literatur}

1. Campbell CM und Edwards RR. Mind-body interactions in pain: the neurophysiology of anxious and catastrophic pain-related thoughts. Transl Res 2009; 153: 97-101.

2. Ceusters W, Michelotti A, Raphael KG, et al. Perspectives on next steps in classification of orofacial pain - part 1: role of ontology. J Oral Rehabil 2015; 42: 926-941

3. Greene CS, Mohl ND, McNeill C, et al. Temporomandibular disorders and science: a response to the critics. J Prosthet Dent 1998; 80: 214-215.

4. Greene CS und Obrez A. Treating temporomandibular disorders with permanent mandibular repositioning: is it medically necessary? Oral Surg Oral Med Oral Pathol Oral Radiol 2015; 119: 489-498.

5. Michelotti A, Alstergren P, Goulet JP, et al. Next steps in development of the diagnostic criteria for temporomandibular disorders (DC/TMD): Recommendations from the International RDC/TMD Consortium Network workshop. J Oral Rehabil 2016; 43: 453-467.

6. Ohrbach R und Greene C. Temperomandibular joint diagnosis: striking a balance between the sufficiency of clinical assessment and the need for imaging Oral Surg Oral Med Oral Pathol Oral Radiol 2013; 116: 124-125.

7. Durham J und Ohrbach R. Commentary on disability and dental education. J Oral Rehabil 2010; 37: 490-494.

8. Ohrbach R und Durham J. Biopsychosocial aspects of orofacial pain. In: CS Farah, R Balasubramaniam and MJ McCullough (eds) Contemporary oral medicine. Heidelberg: Springer Meteor, 2018, S. 1-21.

9. Ohrbach R und Dworkin SF. The evolution of TMD diagnosis: past, present, future. J Dent Res 2016; 95: 1093-1101.

10. Fillingim RB, Bruehl S, Dworkin RH, et al. The ACTTION-American Pain Society Pain Taxonomy (AAPT): an evidence-based and multidimensional approach to classifying chronic pain conditions. J Pain 2014; 15: 241-249.

11. Dworkin SF und LeResche L. Research diagnostic criteria for temporomandibular disorders: review, criteria, examinations and specifications, critique. J Craniomandib Disord 1992; 6: 301-355.
12. Schiffman E und Ohrbach R. Executive summary of the Diagnostic Criteria for Temporomandibular Disorders for clinical and research applications. J Am Dent Assoc 2016; 147: 438-445.

13. Schiffman E, Ohrbach R, Truelove E, et al. Diagnostic Criteria for Temporomandibular Disorders (DC/TMD) for clinical and research applications: recommendations of the International RDC/TMD Consortium Network and Orofacial Pain Special Interest Group. J Oral Facial Pain Headache 2014; 28: 6-27.

14. Ohrbach R und Michelotti A. Psychological considerations. In: S Kandasamy, CS Greene, DJ Rinchuse, et al. (eds) TMD and orthodontics: A clinical guide for the orthodontist. Cham, Switzerland: Springer, 2015, pp.49-61.

15. Ohrbach R, Fillingim RB, Mulkey F, et al. Clinical findings and pain symptoms as potential risk factors for chronic TMD: descriptive data and empirically identified domains from the OPPERA case-control study. J Pain 2011; 12(11, Suppl 3): T27-T45.

16. Durham J, Shen J, Breckons $M$, et al. Healthcare cost and impact of persistent orofacial pain: The DEEP Study Cohort. J Dent Res 2016; 95: 1147-1154.

17. Dworkin SF, Huggins $\mathrm{KH}$, Wilson $\mathrm{L}$, et al. A randomized clinical trial using research diagnostic criteria for temporomandibular disorders-axis II to target clinic cases for a tailored self-care TMD treatment program. J Orofac Pain 2002; 16: 48-63.

18. Dworkin SF, Turner JA, Mancl L, et al. A randomized clinical trial of a tailored comprehensive care treatment program for temporomandibular disorders. J Orofac Pain 2002; 16: 259-276.

19. Von Korff M and Dunn KM. Chronic pain reconsidered. Pain 2008; 138: 267-276.

20. Ohrbach R. Disability assessment in temporomandibular disorders and masticatory system rehabilitation. J Oral Rehabil 2010; 37: 452-480.

21. Ohrbach R, Granger CV, List T, et al. Pain-related functional limitation of the jaw: preliminary development and validation of the Jaw Functional Limitation Scale. Community Dent Oral Epidemiol 2008; 36: 228-236.

22. Ohrbach R, Larsson $P$ and List T. The Jaw Functional Limitation Scale: development, reliability, and validity of 8 -item and 20 -item versions. J Orofac Pain 2008, 22: $219-230$.

23. Kaplan SEF and Ohrbach R. Self-report of waking-state oral parafunctional behaviors in the natural environment. J Oral Facial Pain Headache 2016; 30: 107-119.

24. Markiewicz MR, Ohrbach R and McCall WD Jr. Oral Behaviors Checklist: reliability of performance in targeted waking-state behaviors. J Orofac Pain 2006; 20 : 306-316.

25. Ohrbach R, Markiewicz MR and McCall WD Jr. Waking-state oral parafunctional behaviors: specificity and validity as assessed by electromyography. Eur J Oral Sci 2008; 116: 438-444.

26. Carlsson GE, Egermark I and Magnusson T. Predictors of bruxism, other oral parafunctions, and tooth wear over a 20-year follow-up period. J Orofac Pain 2003; 17: 50-57.

27. Glaros AG and Burton E. Parafunctional clenching, pain, and effort in temporomandibular disorders. J Behav Med 2004; 27: 91-100.

28. Glaros AG, Marszalek JM and Williams KB. Longitudinal multilevel modeling of facial pain, muscle tension, and stress. J Dent Res 2016; 95: 416-422.

29. Glaros AG and Williams K. Tooth contact versus clenching: oral parafunctions and facial pain. J Orofac Pain 2012; 26: 176-180.

30. Ohrbach R, Bair E, Fillingim RB, et al. Clinical orofacial characteristics associated with risk of first-onset TMD: the OPPERA prospective cohort study. J Pain 2013; 14(12, Suppl 2): T33-T50.

31. O'Sullivan P. Diagnosis and classification of chronic low back pain disorders: maladaptive movement and motor control impairments as underlying mechanism. Man Ther 2005; 10: 242-255.

32. Spitzer RL, Kroenke K and Williams JBW. Validation and utility of a selfreport version of PRIME-MD: The PHQ Primary Care Study. JAMA 1999; 282: 1737-1744.

33. Kroenke K, Spitzer RL, Williams JB, et al. The patient health questionnaire somatic, anxiety, and depressive symptom scales: a systematic review. Gen Hosp Psychiatry 2010; 32: 345-359.

34. Durham J, Raphael KG, Benoliel R, et al. Perspectives on next steps in classification of orofacial pain - part 2: role of psychosocial factors. J Oral Rehabil 2015 ; 42: 942-955.

35. Robinson ME, Wise EA, Gagnon C, et al. Influences of gender role and anxiety on sex differences in temporal summation of pain. J Pain 2004; 5: 77-82.

36. Cioffi I, Michelotti A, Perrotta S, et al. Effect of somatosensory amplification and trait anxiety on experimentally induced orthodontic pain. Eur J Oral Sci 2016; 124: 127-134. 
37. Aggarwal VR, McBeth J, Zakrzewska JM, et al. The epidemiology of chronic syndromes that are frequently unexplained: do they have common associated factors? Int J Epidemiol 2006; 35: 468-476.

38. Kirmayer LJ und Robbins JM. Functional somatic syndromes. In: LJ Kirmayer and JM Robbins (eds) Current concepts of somatization: research and clinical perspectives. Washington, DC: American Psychiatric Press, 1991, S. 79-106.

39. Rief W und Broadbent E. Explaining medically unexplained symptoms-models and mechanisms. Clin Psychol Rev 2007; 27: 821-841.

40. Craig AD. How do you feel? Interoception: the sense of the physiological condition of the body. Nat Rev Neurosci 2002; 3: 655-666.

41. Craig AD. A new view of pain as a homeostatic emotion. Trends Neurosci 2003; 26: 303-307.

42. Peters S, Goldthorpe J, McElroy C, et al. Managing chronic orofacial pain: a qualitative study of patients', doctors', and dentists' experiences. Br J Health Psychol 2015; 20: 777-791.

43. Melis M und Zawawi KH. Occlusal dysesthesia: a topical narrative review. J Oral Rehabil 2015; 42: 779-785.

44. Quartana PJ, Campbell CM und Edwards RR. Pain catastrophizing: a critical review. Expert Rev Neurother 2009; 9: 745-758.

45. Brister H, Turner JA, Aaron LA, et al. Self-efficacy is associated with pain, functioning, and coping in patients with chronic temporomandibular disorder pain. J Orofac Pain 2006; 20: 115-124.

46. Litt MD und Porto FB. Determinants of pain treatment response and nonresponse: identification of TMD patient subgroups. J Pain 2013; 14: 1502-1513.

47. Turner JA, Brister $\mathrm{H}$, Huggins $\mathrm{KH}$, et al. Catastrophizing is associated with clinical examination findings, activity interference, and health care use among patients with temporomandibular disorders. J Orofac Pain 2005; 19: 291-300.

48. Sullivan MJL, Bishop SR und Pivik J. The Pain Catastrophizing Scale: development and validation. Psychol Assess 1995; 7: 524-532.

49. Harland NJ und Georgieff K. Development of the Coping Strategies Questionnaire 24 , a clinically utilitarian version of the coping strategies questionnaire. Rehabil Psychol 2003; 48: 296-300.

50. Fordyce WE. Behavioral methods for chronic pain and illness. St Louis, MO: CV Mosby, 1976.

51. Vlaeyen JW und Linton SJ. Fear-avoidance and its consequences in chronic musculoskeletal pain: a state of the art. Pain 2000; 85: 317-332.

52. Wall PD. On the relation of injury to pain. Pain 1979; 6: 253-264.

53. Kori SH, Miller RP and Todd DD. Kinesiophobia: a new view of chronic pain behavior. Pain Manag 1990; 3: 35-43.

54. Boersma K und Linton SJ. Expectancy, fear and pain in the prediction of chronic pain and disability: a prospective analysis. Eur J Pain 2006; 10: 551-557.

55. Visscher CM, Ohrbach R, van Wijk AJ, et al. The Tampa Scale for Kinesiophobia for Temporomandibular Disorders (TSK-TMD). Pain 2010; 150: 492-500.

56. Spencer CJ, Neubert JK, Gremillion H, et al. Case reviews in pain: toothache or trigeminal neuralgia: treatment dilemmas. J Pain 2008; 9: 767-770.

\section{Glossar zur ICOP [1]}

Allodynie Ein Schmerzempfinden, das durch einen Reiz ausgelöst wird, der normalerweise nicht ausreichen würde, um diese Wirkung zu haben. Es handelt sich um einen klinischen Begriff, der nichts über einen beteiligten Mechanismus aussagt.

Anhaltender Schmerz Innerhalb der ICHD bezeichnet dieser Begriff im Kontext bestimmter sekundärer Kopfschmerzen einen initial akuten und von einer anderen Erkrankung verursachten Kopfschmerz, der nicht innerhalb eines spezifizierten Zeitraums (üblicherweise 3 Monate) nach Genesung von dieser Erkrankung verschwindet. In vielen Fällen dieser Art wird der Kopfschmerz als klar umrissener Subtyp oder klar umrissene Unterform anerkannt, wobei der Nachweis des ursächlichen Zusammenhangs davon abhängt, dass zuvor die Kriterien für eine Diagnose des akuten Typs erfüllt waren und dass derselbe Kopfschmerz anhält. In der ICOP jedoch hat im Rahmen der Typen der 6. Idiopathischen orofazialen Schmerzen der Begriff anhaltend die Bedeutung von chronisch - in dem Sinne, dass der Schmerz mehr als 3 Monate lang vorliegt oder wiederkehrt. Diese Begriffsverwendung findet sich auch in der ICHD.

Attackendauer Zeit vom Beginn bis zum Ende einer Schmerzattacke, die die Kriterien für einen bestimmten Schmerztyp oder -subtyp erfüllt. Schläft ein Patient während einer Attacke ein und erwacht schmerzfrei, zählt die Zeit bis zum Aufwachen mit zur Attackendauer.

Chronisch In der Schmerzterminologie bedeutet chronisch lange anhaltend oder, konkreter, ein Anhalten über einen Zeitraum von mehr als 3 Monaten. In der Kopfschmerzterminologie wird der Begriff chronisch bei den primären Kopfschmerzerkrankungen, die häufiger episodisch auftreten, immer dann verwendet, wenn der Kopfschmerz über einen Zeitraum von mehr 3 Monaten hinweg an mehr als der Hälfte der Tage auftritt. Eine Ausnahme sind die trigeminoautonomen Kopfschmerzerkrankungen. Hier wird erst von chronischen Verläufen gesprochen, wenn die Erkrankung über mehr als ein Jahr nicht remittiert ist und der Patient weniger als 3 Monate attackenfrei geblieben ist. Inwieweit sich diese Definitionen auf orofaziale Schmerzen anwenden lassen, werden Untersuchungen auf Grundlage der ICOP [2] zeigen.

Dysästhesie Eine unangenehme, abnorme Missempfindung, ob spontan oder evoziert. Zu den Sonderfällen der Dysästhesie gehören die Hyperalgesie und Allodynie. Von einer "Dysästhesie" sollte nur dann die Rede sein, wenn es sich um eine unangenehme Sensibilitätsstörung handelt, anders als bei der Parästhesie, wenngleich die Abgrenzung zugegebenermaßen schwierig sein kann.

Episodisch Nach einem regelmäßigen oder unregelmäßigen Zeitmuster wiederkehrende und remittierende Schmerzattacken von einheitlicher oder variierender Dauer. In der Praxis hat dieser schon lange gebrauchte Begriff beim episodischen Clusterkopfschmerz eine besondere Bedeutung erfahren. Hier wird mit episodisch nicht das Auftreten von einzelnen Attacken bezeichnet, sondern von Clusterepisoden, die durch Remissionsphasen getrennt sind. In einem ähnlichen Sinne wurde der Begriff für die Subtypen von 5.3 Trigeminoautonome orofaziale Schmerzen übernommen.

Gesichtsschmerz Schmerz unterhalb der Orbitomeatallinie, oberhalb des Halses und vor der Ohrmuschel.

Hypästhesie Herabgesetzte Sensibilität in Reaktion auf einen Reiz, ausgenommen Sinne (Schmecken, Sehen, Hören, Riechen). Hier sind der betroffene Reiz und der Bereich anzugeben, in dem die Hypästhesie auftritt.

Hypalgesie Herabgesetzte Schmerzempfindung in Reaktion auf einen Reiz, der erwartungsgemäß schmerzhaft sein müsste. Es handelt sich um einen klinischen Begriff, der nichts über einen beteiligten Mechanismus aussagt.

Hyperalgesie Gesteigerte Schmerzempfindung in Reaktion auf einen Reiz, der erwartungsgemäß schmerzhaft sein müsste. Der Begriff bezeichnet ein erhöhtes Schmerzempfinden bei überschwelligen Reizen und ist eine klinische Bezeichnung, die nichts über einen beteiligten Mechanismus aussagt. Hyperalgesie wird von einem Schmerzempfinden unterschieden, das durch einen Reiz ausgelöst wird, der erwartungsgemäß nicht schmerzhaft wäre, und für das vorzugsweise der Begriff Allodynie verwendet wird.

Hyperästhesie Gesteigerte Empfindlichkeit auf Reize, ausgenommen Sinne (Schmecken, Sehen, Hören, Riechen). Hier sind der betroffene Reiz und der Bereich anzugeben, in dem die Hyperästhesie auftritt. Diese kann sich auf verschiedene Arten kutaner Sensibilität beziehen, darunter eine erhöhte Empfindlichkeit der Haut gegen Berührung und thermische Wahrnehmungen ohne Schmerz wie auch gegen Schmerz. Der Begriff wird sowohl für eine generell verminderte Reiz-Reaktions-Schwelle verwendet als auch für eine übersteigerte Reaktion auf sonst als normal erachtete Reize.

Kopfschmerz Schmerz im Kopf oberhalb der Orbitomeatallinie und/oder der Linea nuchae.

Neuralgie Schmerz im/in den Verteilungsgebiet/en eines Nervs oder mehrerer Nerven. Im allgemeinen Sprachgebrauch impliziert der Begriff - vor allem in Europa -oft eine paroxysmale Schmerzqualität, doch sollte der Begriff Neuralgie nicht paroxysmalen Schmerzen vorbehalten sein.

Neuritis Entzündlicher Prozess in einem Nerv oder mehreren Nerven.

Neuropathie Eine funktionelle Störung oder pathologische Veränderung in (einem) Nerv(en). Eine Sonderform der Neuropathie ist die Neuritis, wobei dieser Begriff sich auf entzündliche Prozesse beschränkt, die Auswirkungen auf Nerven haben.

Neuropathischer Schmerz Schmerz infolge einer Läsion oder Erkrankung des somatosensorischen Nervensystems. Bei dem Begriff handelt es sich um eine klinische Beschreibung: im Rahmen einer Diagnose angewandt, setzt er eine nachweisbare Läsion oder Erkrankung voraus, die anerkannten neurologischen Diagnosekriterien genügt.

Nozizeption Der neuronale Prozess der Enkodierung von Schadreizen.

Nozizeptiver Schmerz Schmerz, der aus einer tatsächlichen oder drohenden Schädigung von nichtneuronalem Gewebe resultiert und auf die Aktivierung von Nozizeptoren zurückgeht. Dieser Begriff, der für Schmerzen verwendet wird, die in einem normal funktionierenden somatosensorischen Nervensystem auftreten, dient der Abgrenzung von dem abnormalen Funktionieren, das bei neuropathischen Schmerzen vorliegt.

Nozizeptor Ein hochschwelliger sensorischer Rezeptor des peripheren somatosensorischen Nervensystems, der in der Lage ist, Schadreize umzuwandeln und zu enkodieren.

Noziplastischer Schmerz Schmerz infolge einer veränderten Nozizeption ohne eindeutigen Nachweis einer tatsächlichen oder drohenden Gewebeschädigung, die ursächlich für die Aktivierung peripherer Nozizeptoren ist, oder ohne Nachweis einer für den Schmerz ursächlichen Erkrankung oder Läsion des somatosensorischen Systems. 
Parästhesie Eine abnormale Körperempfindung, ob spontan oder evoziert. Es wird empfohlen, Parästhesie zur Beschreibung einer abnormalen Empfindung zu verwenden, die nicht unangenehm ist und Dysästhesie für eine abnormale Empfindung, die als unangenehm betrachtet wird.

Peripherer neuropathischer Schmerz Neuropathischer Schmerz, der durch eine Läsion oder Erkrankung des peripheren somatosensorischen Nervensystems verursacht wird.

Periphere Sensibilisierung Eine Sensibilisierung unter Beteiligung nozizeptiver Neuronen in der Peripherie.

Phonophobie Überempfindlichkeit gegenüber Geräuschen, selbst bei normaler Lautstärke, die gewöhnlich zu einem Vermeidungsverhalten führt.

Photophobie Überempfindlichkeit gegenüber Licht, selbst bei normaler Helligkeit, die gewöhnlich zu einem Vermeidungsverhalten führt.

Primäre(r) Schmerz(-erkrankung/-störung) Schmerz (orofazialer Schmerz oder Kopfschmerz), der nicht durch eine andere Störung verursacht oder auf eine solche zurückzuführen ist. Diese(r) wird von einer sekundären Schmerzerkrankung unterschieden.

Refraktärzeit Die Zeit nach dem Verschwinden/der Resolution einer Schmerzattacke, während der keine weitere Attacke ausgelöst werden kann.

Schadreiz Ein Reiz, der normales Gewebe schädigt oder zu schädigen droht.

Schmerz Eine unangenehme sensorische und emotionale Erfahrung in Verbindung mit einer tatsächlichen oder potenziellen Gewebeschädigung oder die in Bezug auf eine solche Schädigung beschrieben wird.

Schmerzfrequenz Die Häufigkeitsrate von Schmerzattacken in einem bestimmten Zeitraum (meist 1 Monat).

Schmerzintensität Schmerzgrad, in der Regel auf einer numerischen 4-PunkteSkala beschrieben (0 bis 3), gleichbedeutend mit "kein Schmerz", ${ }_{\text {,leicht }}$, "mittelstark" und „stark", oder auf einer visuellen Analogskala (üblicherweise $10 \mathrm{~cm}$ ). Kann auch nach einer verbalen Punkte-Skala von 0-10 bewertet werden oder gemäß den funktionellen Konsequenzen: 0 = kein Schmerz; 1 = leichter Schmerz, behindert normale Aktivitäten nicht; 2 = mittelstarker Schmerz, beeinträchtigt zwar normale Aktivitäten, aber verhindert sie nicht; 3 =starker Schmerz, verhindert alle Aktivitäten.

Sekundäre(r) Schmerz(-erkrankung/-störung) Schmerz (orofazialer Schmerz oder Kopfschmerz), der durch eine andere Störung verursacht oder auf eine solche zurückzuführen ist. Diese(r) wird von einer primären Schmerzerkrankung/-störung unterschieden.

Sensibilisierung Erhöhte Empfänglichkeit nozizeptiver Neuronen auf normale Reize und/oder Verstärkung einer Reaktion auf normalerweise unterschwellige Reize.

Unilateral Entweder links- oder rechtsseitig, die Mittellinie nicht überquerend. Wird der Begriff für sensorische oder motorische Störungen im Rahmen einer Migräneaura benutzt, schließt er sowohl eine komplette als auch eine nur teilweise Halbseitenbeteiligung mit ein.

Zentraler neuropathischer Schmerz Ein neuropathischer Schmerz, der durch eine Läsion oder Erkrankung des zentralen somatosensorischen Nervensystems verursacht wird.

Zentrale Sensibilisierung Eine Sensibilisierung, bei der nozizeptive Neuronen im Zentralnervensystem beteiligt sind.

Zurückzuführen auf Dieser Begriff wird in der ICOP in Übereinstimmung mit der ICHD-3 [3] verwendet und beschreibt die Beziehung zwischen einem sekundären Schmerz und der Erkrankung, die als dessen Ursache angenommen wird. Voraussetzung hierfür ist, dass die Kriterien erfüllt sind, die hinreichend als Nachweis eines ursächlichen Zusammenhangs anerkannt sind.

\section{Literatur}

1. International Association for the Study of Pain. IASP Terminology, https://www. iasp-pain.org/Education/Content.aspx?ItemNumber 1698 (2017, aufgerufen Januar 2020)

2. Benoliel R, Eliav E und Sharav Y. Classification of chronic orofacial pain: applicability of chronic headache criteria. Oral Surg 2010; 110: 729-737.

3. Headache Classification Committee of the International Headache Society. The International Classification of Headache Disorders, 3. Auflage (ICHD-3). Cephalalgia 2018; 38: 1-211. 
Hier steht eine Anzeige.

\section{Springer}

